\title{
NاكT
}

\section{Standard Reference Materials Catalog 1990-91}

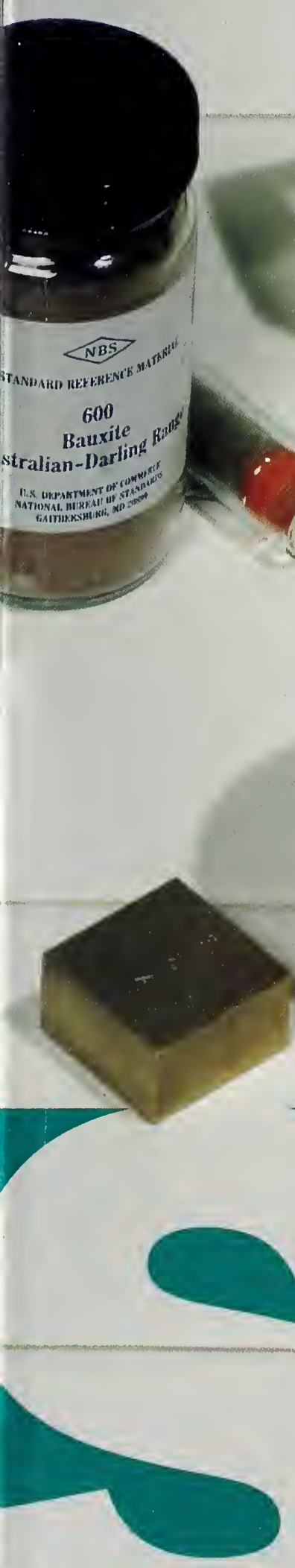




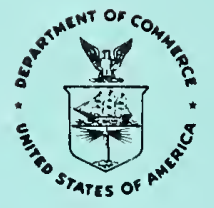

UNITED STATES DEPARTMENT OF COMMERCE

National Institute of Standards and Technology

[formerly National Bureau of Standards]

Gathersburg, Maryland 20899

\section{Dear Colleague:}

Welcome to the very first catalog of Standard Reference Materials (SRM's) to be issued by the National Institute of Standards and Technology. Of course, the materials listed herein are an extension of 84 years of service to the U.S. technical community provided by the National Bureau of Standards since 1906. Our new name is indicative of the effort we are making to extend the tradition of SRM service into new fields and into a new era of service to technology.

You will find over two hundred changes in SRM availability listed in this catalog — new additions, renewals, and revised certificates. Please carefully review the sections which relate to your work to see the new (and previously issued) SRM's that are available. If we can help you with regard to any listing please give us a call.

Many of you have told us, at SRM exhibits, or by letter, that you are happy that SRM's continue to be available through the new NIST organization. In fact, we are seeing some modest growth in our ability to supply the materials that you need. If you have a requirement that we do not currently meet please let us know about it. In the front pages of this catalog you will find a section entitled Guide for Requesting Development of Standard Reference Materials. You can register your need with us with just a few minutes of effort. Then, as funding permits, we can try to be responsive to your needs.

Thank you for your continued interest in Standard Reference Materials from NIST. It is our privilege to provide them to you and thereby enhance the quality of measurements and analyses made throughout the technical community.

Sincerely,
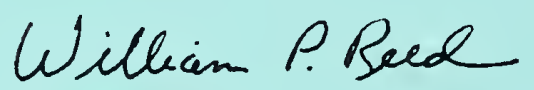

William P. Reed

Acting Chief

Office of Standard Reference Materials 


\section{NIST Standard \\ Reference Materials Catalog 1990 - 1991}

\section{R.L. McKenzie, Editor}

Office of Standard Reference Materials

National Institute of Standards and Technology

Gaithersburg, MD 20899

CAUTION: The values shown in the catalog are nominal values only. Users should consult the certificate issued with an SRM for the certified values.

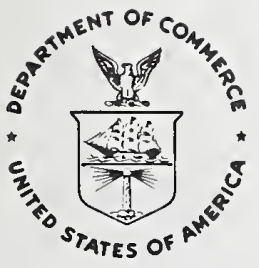

U.S. Department of Commerce

Robert A. Mosbacher, Secretary

National Institute of Standards and Technology

Ramond G. Kammer, Acting Director 
National Institute of Standards and Technology

Special Publication 260

Supersedes NIST Spec. Publ. 260, 1988-89

163 pages (January 1990)

CODEN: XNBSAV

\section{U.S. GOVERNMENT PRINTING OFFICE}

WASHINGTON: 1990

For sale by the Superintendent of Documents

U.S. Government Printing Office, Washington, DC 20402 


\section{Contents}

\section{ABSTRACT AND KEY WORDS, 1}

PROGRAM INFORMATION, 3

Definitions, 3

SRM Catalog, 4

Preparation and Availability of Standard Reference Materials, 4

Guide for Requesting Development of Standard Reference Materials, 5

\section{ORDERING STANDARD REFERENCE}

MATERIALS, 6

General, 6

Terms, 7

Late Charges, 8

Proforma Invoice (Price Quotations), 8

Domestic Shipments, 8

Foreign Shipments, 9

Documentation, 9

\section{CERTIFIED REFERENCE MATERIALS}

FROM OTHER SOURCES, 10

Special Nuclear Materials, 10

International CRM's, 10

\section{OTHER SERVICES OF THE NATIONAL}

INSTITUTE OF STANDARDS AND

TECHNOLOGY, 11

Calibration and Related Measurement Services, 11

Office of Weights and Measures, 11

Proficiency Sample Programs, 12

Accreditation of Testing Laboratories, 12

National Center for Standards and Certification Information, 12

National Standard Reference Data System, 12

\section{CHEMICAL COMPOSITION, 15}

Ferrous Alloys, 15

Steels (Chip Form), 15

Plain Carbon Steels, 15

Low Alloy Steels, 16

Special Low Alloy Steels, 17

High Alloy Steels, 18

Stainless Steels, 19

Tool Steels, 19

Steels (Solid Form), 20

Low Alloy Steels, 20

Stainless Steels, 24

Specialty Steels, 24

High Temperature Alloys, 25

Steelmaking Alloys, 26
Cast Irons (Chip Form), 27

Cast Steels, White Cast Irons, Ductile

Irons, and Blast Furnace Irons (Solid Form), 28

Nonferrous Alloys, 30

Aluminum-Base Alloys, 30

Copper-Base Alloys (Chip Form), 31

Copper-Base Alloys (Solid Form), 32

Copper "Benchmark", 33

Lead-Base Alloys, 34

Lead-Base Material, 34

Nickel-Base Alloys, 35

Carbon-Modified Silicon, 35

Trace Elements in Nickel-Base Superalloys (Chip Form), 36

Nickel Oxides (Powder Form), 36

Titanium-Base Alloys (Chip Form), 37

Zinc-Base Alloys, 38

Zirconium-Base Alloys, 38

Gases in Metals, 39

High Purity Metals, 40

Microanalytical, 41

Metals for Microanalysis, 41

Mineral Glasses for Microanalysis, 42

Glasses for Microchemnical Analysis, 42

Thin Film for X-Ray Spectrometry, 43

Glass Fibers for Microanalysis, 43

Primary, Working, and Secondary Chemicals, 44

Microchemicals, 44

Spectrometric Solutions, 45

Anion Ion Chromatographic Solutions, 48

Clinical Laboratory, 49

Serum Reference Materials, 50

Biological Materials, 51

Food and Beverage, 51

Ethanol Solutions, 52

Agriculture, 53

Environmental Materials, 54

Analyzed Gases, 54

Permeation Devices, 57

Analyzed Liquids and Solids, 59

Simulated Rainwaters, 59

Alcohols in Reference Fuels, 60

Sulfur in Fossil Fuels, 61

Trace Elements, 62

Organic Constituents, 63

Industrial Hygiene, 68

Freeze-Dried Urine, 68

Thin Films for X-ray Fluorescence, 69

Materials on Filter Media, 69

Blank Filters, 69

Respirable Quartz, 70

Asbestos, 71 
Lubricating Materials, 72

Metallo-Organic Compounds, 72

Lubricating Base Oils, 72

Catalyst Package for Lubricant Oxidation, 73

Wear-Metals in Oil, 73

Fertilizers, 74

Ores, 75

Rocks, Minerals, and Refractories, 79

Clays, 78

Mercury in Soil, 79

Carbides, 81

Glasses, 83

Cements, 84

Portland Cement Clinkers, 85

Trace Elements, 86

Nuclear Materials, 87

Radiation Dosimetry, 87

Fission Track Glasses, 87

Stable Isotopic Materials, 88

PHYSICAL PROPERTIES, 89

Ion Activity, 89

$\mathrm{pH}, 89$

$\mathrm{pD}, 89$

Ion-Selective Electrodes, 90

Metrology, 90

Scanning Electron Microscope, 90

Optical Microscope Linewidth-Measurement, 91

Depth Profiling, 91

Coating Thickness, 92

Nonmagnetic Coating on Magnetic Substrate, 92

Magnetic Coating on Magnetic Substrate, 92

Solder Thickness, 92

Coating Weight, 93

Gold Coating on Nickel, 93

Ellipsometry, 93

Glass, 94

Chemical Resistance (Durability) of Glass, 94

Electrical Properties of Glass, 94

Viscosity, 94

Viscosity Fixpoints, 95

Relative Stress Optical Coefficient, 95

Glass Liquidus Temperature, 95

Density, 96

Microhardness, 96

Ultrasonics, 97

Polymers, 97

Molecular Weight (Melt Flow), 97

Rheology, 98

Heat, 99

Calorimetric, 99

Combustion, 99

Solution, 99

Heat Source, 99

Enthalpy and Heat Capacity, 100

Differential Scanning Calorimetry, 100

Differential Thermal Analysis, 101

Superconductive Thermometric Fixed Point Devices, 101

Freezing Point Materials, 102

Defining Fixed Points, 102

Secondary Reference Points, 102
Melting Point, 102

Laboratory Thermometer, 103

Thermocouple Material, 103

Vapor Pressure, 103

Thermal Conductivity, 104

Thermal Expansion, 104

Thermal Resistance, 104

Magnetic, 105

Magnetic Susceptibility, 105

Magnetic Moment, 105

Optical, 106

Spectrophotometric, 106

Reflectance, 107

Specular Spectral Reflectance, 107

Infrared Reflectance, 107

Directional-Hemispherical Reflectance, 108

Refractive Index, 108

Optical Rotation, 108

Radioactivity, 109

Alpha-particle, Beta-particle, Gamma-ray, and Electron-capture Solutions, 110

Alpha-Particle Point-Sources, 111

Radiocarbon Dating and Ground Water Studies, 111

Gaseous Materials, 111

Gamma-ray and X-ray Point-Sources, 112

Low-Energy Photon Point-Sources, 112

Radium-226 Solutions, 112

Radon Analysis, 112

Gamma-ray Solutions, 113

Environmental Natural Matrix Materials for Quality Assurance Testing, 113

Radiopharmaceuticals, 114

Metallurgical, 115

Abrasive Wear, 115

Corrosion, 115

Electrochemical Potential and Thickness, 115

Pitting or Crevice Corrosion, 116

$\mathrm{X}$-ray Fluorescent Emission Target, 116

X-ray Diffraction, 116

Gas Transmission, 117

Reference Fuels, 118

Electrical Resistivity and Conductivity, 118

Metals, 118

Silicon, 118

Residual Resistivity Ratio, 119

Eddy Current, 119

Electrolytic Conductance, 119

Superconducting Critical Current, 119

ENGINEERING MATERIALS, 121

Standard Rubbers and Rubber-Compounding Materials, 121

Sizing, 122

Particle Size, 122

Cement Turbidimetric and Fineness, 122

Surface Area of Powders, 123

Performance Standards, 123

Socketed Ball Bar, 123

Dye Penetrant Test Blocks, 123

Surface Roughness, 124

Charpy V-Notch Test Blocks, 124

Artificial Flaw for Eddy Current NDE, 124 
Color, 125

$\mathrm{X}$-ray and Photographic, 125

Magnetic Computer Storage Media, 125

Centerline Drawings for Optical Character

Recognition, 126

NIST Time Software, 127

Fire Research, 128

Surface Flammability, 128

Smoke Density Chamber, 128

Flooring Radiant Panel, 128

Tape Adhesion Testing, 128

ADDITIONAL INFORMATION, 129

NIST Special Publications in the 260 Series, 129

Calibration Service Contacts, 133

\section{INDICES, 135}

Numerical Index of Standard Reference Materials (Name and Certificate Date), 135

Alphabetical Index by Standard Reference

Material (Name and Category), 145

\section{Program and \\ Sales Information}

\section{Chemical Composition 15}

Physical Properties

89

Engineering Materials 



\section{Abstract and Key Words}

\section{National Institute of Standards and Technology Standard Reference Materials 1990-91 Catalog}

This catalog describes the Standard Reference Materials (SRM's) currently available from the National Institute of Standards and Technology (NIST), lists those in preparation, and provides ordering information. The descriptions provide nominal values for these SRM's. Certified values are provided in the certificates that accompany each SRM. Price Lists for SRM's are issued as separate supplements to this catalog and include new SRM's as they are issued.

Key Words: analysis, calibration, characterization, composition, concentration, materials, measurement, property, quality assurance, quality control, reference materials, Standard Reference Materials, standardization.

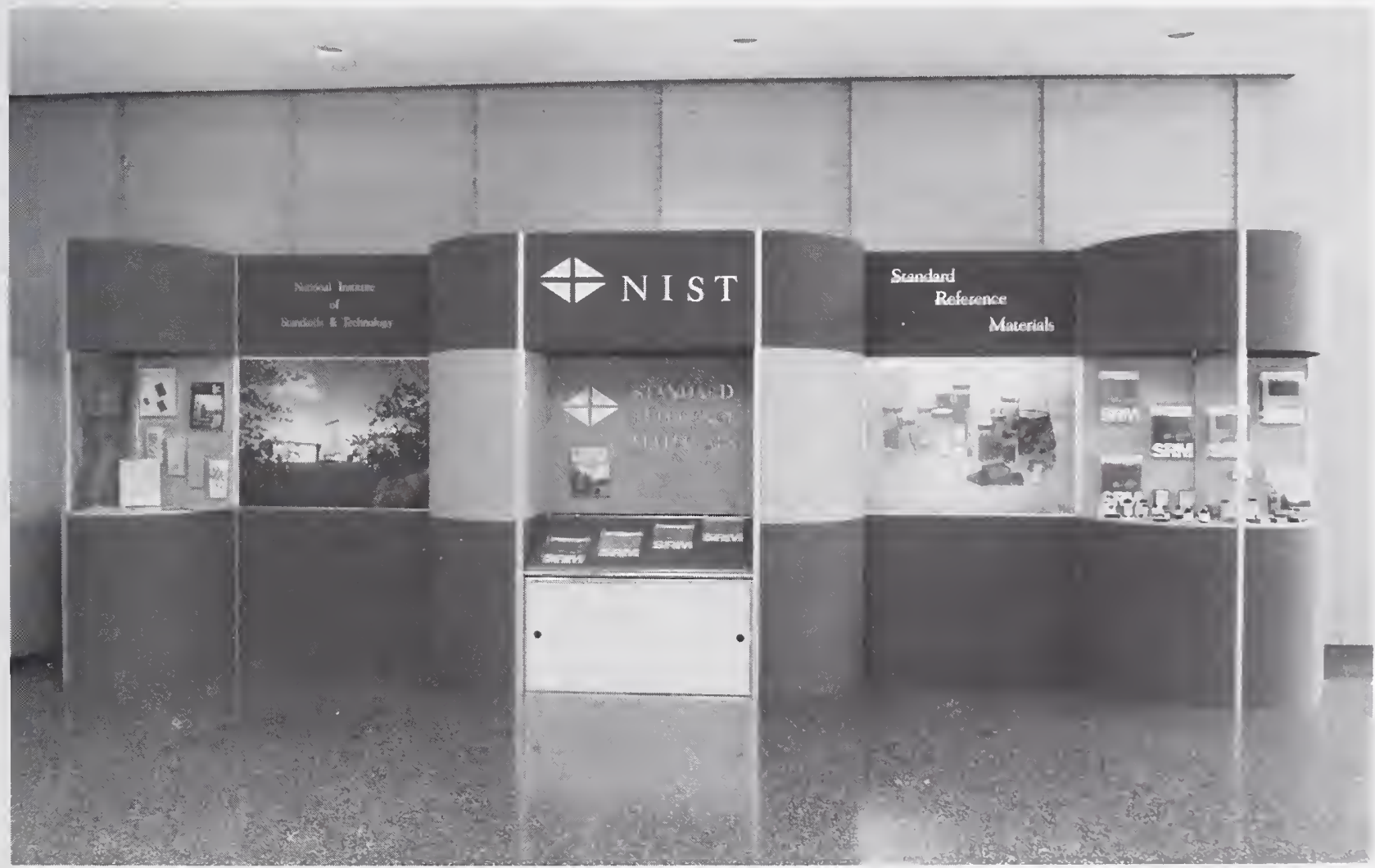

Look for us at the next meeting you attend! Our new exhibit booth, attended by representatives of our sales and technical staff, is scheduled to be at about a dozen professional meetings and expositions each year. 
The Engineering Mechanics Building is now the

home of the Standard Reference Materials

Program. We look forward to continued and

improved service to the technical community in

our new offices and materials preparation and

packaging facilities.

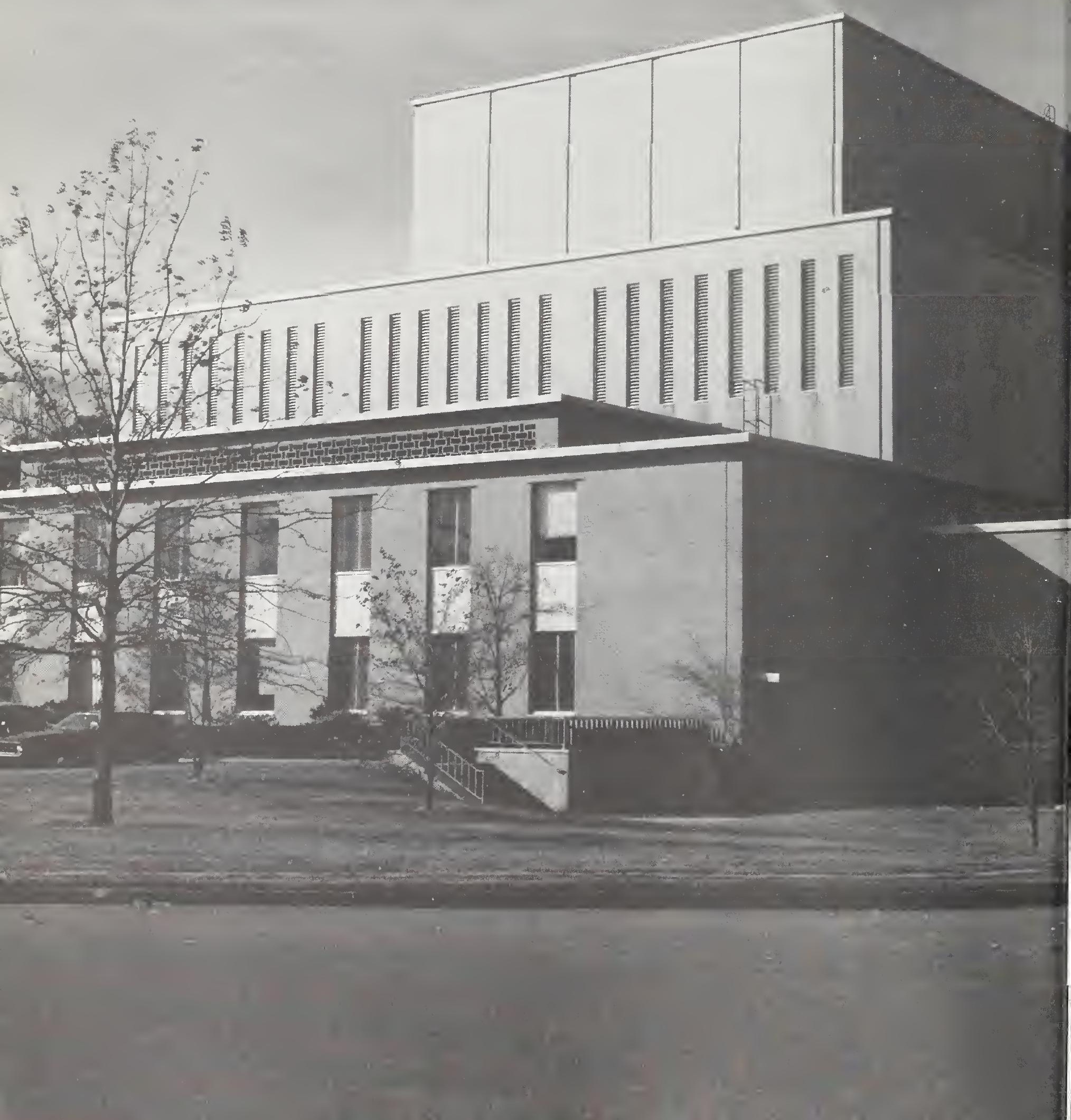




\section{Program Information}

The National Institute of Standards and Technology (NIST) offers for sale over 1,000 different materials through its Office of Standard Reference Materials. These materials are primarily Standard Reference Materials (SRM's) certified for their chemical composition, chemical property, or physical property, but include other reference materials. All materials bear distinguishing names and numbers by which they are permanently identified. Thus, each material bearing a given description is identical (within the specified limits) to every other sample bearing the same designation-with the exception of individually certified items, which are further identified by serial number.

\section{Definitions}

From "Terms and definitions used in connection with reference materials," ISO Guide 30-1981 (E):

1. "Reference Material (RM): A material or substance one or more properties of which are sufficiently well established to be used for the calibration of an apparatus, the assessment of a measurement method, or for assigning values to materials."

2. "Certified Reference Material (CRM): A reference material one or more of whose property values are certified by a technically valid procedure, accompanied by or traceable to a certificate or other documentation which is issued by a certifying body."

NIST Standard Reference Materials (SRM's): Certified reference materials issued by NIST. These are wellcharacterized materials produced in quantity to improve measurement science. SRM's are certified for specific chemical or physical properties, and are issued by NIST with certificates that report the results of the characterization and indicate the intended use of the material. They are prepared and used for three main purposes:

(1) To help develop accurate methods of analysis (reference methods);

(2) To calibrate measurement systems used to:

(a) facilitate exchange of goods,

(b) institute quality control,

(c) determine performance characteristics, or

(d) measure a property at the state-of-the-art limit; and

(3) To assure the long-term adequacy and integrity of measurement quality assurance programs.

NIST certified values are obtained by one of three routes of measurement:

(1) A previously validated reference method,

(2) Two or more independent, reliable measurement methods, or

(3) A network of cooperating laboratories, technically competent and thoroughly knowledgeable with the material being tested.

These measurement routes are described in "The Role of Standard Reference Materials in Measurement Systems," NBS Monograph 148, 54 pages (Jan 1975).

Reference Materials (RM's) listed in this catalog are sold by, but not certified by, NIST. They meet the ISO definition for RM's, and many meet the definition for CRM's. The documentation issued with these materials is either a:

(1) "Report of Investigation," the sole authority of which is the author of the report. RM's are intended to further scientific or technical research on that particular material. The principal consideration in issuing an RM is to provide a homogeneous material so that investigators in different laboratories are assured that they are investigating the same material.

(2) "Certificate," issued by the certifying agency (other than NIST), e.g., other national laboratories, other government agencies, other standardizing bodies, or other non-profit organizations. When deemed to be in the public interest and when alternate means of national distribution do not exist, NIST acts as the distributor for such materials. This service is available to organizations that qualify and have the reference materials that would help meet national measurement needs. 


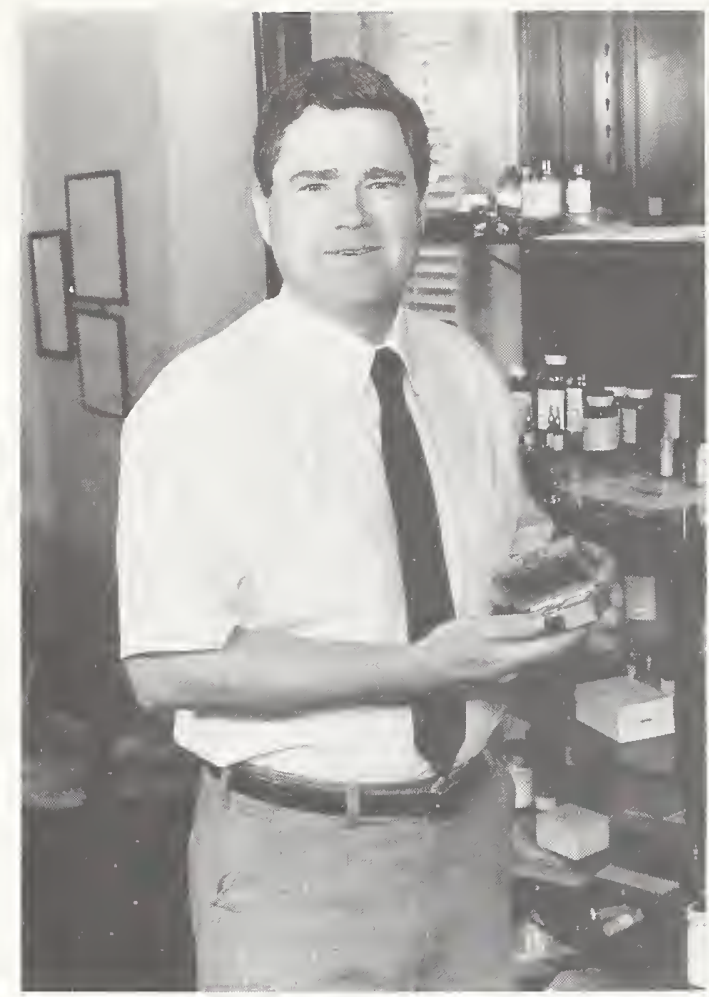

Stan Rasberry, the new Acting Director of Measurement Services, displays one of the many SRM's developed in the program of the Office of Standard Reference Materials (OSRM). The enthusiasm and decisiveness Stan exercised while Chief of OSRM will continue to be an asset to the SRM program as well as to the additional programs to which he will now provide leadership - Standard Reference Data, Physical Measurement Services and the National Voluntary Laboratory Accreditation Programs.

\section{SRM Catalog}

New catalogs of NIST Standard Reference Materials are published approximately every 2 years, listing materials available and materials in preparation, and deleting discontinued materials. Catalog supplements (Price Lists) are issued simultaneously with new catalogs and approximately every 6 months to keep the catalog current between editions. These supplements list current prices, and reflect any changes in material availability-listing new and renewed materials and dropping discontinued ones.

The numerical values given in this catalog to describe the materials' properties are NOMINAL values only and are to be used only as guides in selecting SRM's. They are NOT TO BE USED in place of the values given on the certificate issued with the materials.

Two indices are provided for user convenience. The first is a numerical index that lists the numbers, names, and certificate dates of the materials in the catalog. The second is an alphabetical index that lists categories of materials, elements, and names of materials.

\section{Preparation and Availability of Standard Reference Materials}

New and renewal SRM's are being prepared continually. These SRM's are included in the next edition of the catalog and its supplements. Prospective users that have requested that their names be added to the SRM mail list are notified as these new items become available. To have your name placed on this mail list, please write to the address given below.

Renewal SRM's are intended to be completed before the supply of an existing SRM is exhausted. This is not always possible and an SRM may be out-of-stock for a time. When this occurs, those ordering the material are so notified and possible substitutes (if any) are suggested. When a renewal is issued, customers who have ordered the previous lot are promptly notified of the price and availability of the renewal. If little demand exists or if an alternate source of supply becomes available, production of an SRM may be discontinued permanently.

Renewal SRM's are not identical to the preceeding lot; however, they meet the same specifications and can be used for the same purpose. For example, the first 0.1 percent carbon Bessemer steel was prepared in 1909 (Standard Sample No. 8). Since then a number of renewals, 8a, 8b, 8c, etc., were prepared. The current SRM 8j, Bessemer Steel (Simulated), 0.1\% C, represents the eleventh lot of the material. Each lot differs somewhat in detailed analysis, thus the use of the specific certificate for that lot is essential. 


\section{Guide for Requesting Development of Standard Reference Materials}

The National Institute of Standards and Technology has the function to develop, produce, and distribute Standard Reference Materials (SRM's) that provide a basis for comparison of measurements on materials, and that aid in the control of production processes. To perform this function, the Office of Standard Reference Materials evaluates the requirements of science, industry, and government for carefully characterized reference materials, and directs their production and distribution.

NIST currently has over 1,000 SRM's available, about 100 new ones in preparation, and requests for the production of many others.

To be an SRM, a candidate material must meet one or more of these criteria:

1. It would permit users to attain more accurate measurements.

2. Its production elsewhere would not be economically or technically feasible.

3. It would be an industry-wide standard for commerce from a neutral source not otherwise available.

4. Its production by NIST would provide continued availability of a well-characterized material important to science, industry, or government.

NIST has recognized and responded to requests to enlarge the scope of the SRM program to include all types of well-characterized materials for use in calibrating measurement systems, or for producing scientific data that can be referred to a common base. However, the requests for new SRM's greatly exceed the Institute's capacity to produce and certify such materials. Consequently, requests for new SRM's of limited use, or for which the need is not very great, are deferred in favor of requests that clearly show a critical need. To determine which requests receive top priority, NIST needs and uses information supplied by industry and such interested organizations as the American National Standards Institute, American Nuclear Society, American Petroleum Institute, American Society for Testing and Materials, etc.

Accordingly, while NIST welcomes all requests for developing new SRM's, both NIST and industry would be helped if such requests provide information that permit objective assessment of the urgency and importance of the proposed new reference materials.

Requests for the development of new Standard Reference Materials should provide information such as listed below.

1. Short title of the proposed SRM.

2. Purpose for which the SRM would be used.

3. Reasons why the SRM is needed.

4. Special characteristics and requirements for the material. Include additional requirements and reasons if more than one SRM is necessary for standardization in this area.

5. An estimate of the probable present and future (6-10 year) demand for such an SRM in your operations and elsewhere. (National and international estimates are useful.)

6. Whether such an SRM, or a similar one, could be produced or obtained from a source other than NIST; and if so, justify its preparation by NIST.

7. Miscellaneous pertinent information to aid justification for the SRM, such as: (a) an estimate of the potential range of application, monetary significance of the measurement affected, scientific and technological significance including, when feasible, estimates of the impact upon industrial productivity, growth, quality assurance or control, and (b) supporting letters from industry leaders, trade organizations, interested committees, and others.

All such requests should be addressed to:

Office of Standard Reference Materials

ATTN: SRM Development

Room 205, Building 202

National Institute of Standards and Technology

Gaithersburg, MD 20899 


\section{Ordering Standard Reference Materials}

\section{General}

Purchase orders for all SRM's should be addressed to:

Office of Standard Reference Materials

Room 205, Building 202

National Institute of Standards and Technology

Gaithersburg, MD 20899

Telephone: (301) 975-OSRM [6776]

FTS: 879-OSRM [6776]

Fax number: (301) 948-3730

Telex: TRT197674NIST UT

All orders should give the number of units, catalog number, and name of the material requested. For example: "1 each, SRM 79a, Fluorspar (Customs Grade)." The materials described in this catalog are sold only in the units listed or multiples thereof.

Acceptance of an order does not imply acceptance of any provisions set forth in the order contrary to the policy, practice, or regulations of the National Institute of Standards and Technology or the U.S. Government.

In general, orders received for "out-of-stock" material will be filled with the renewal material, if available; otherwise they will be canceled. Customers are notified when an order is canceled; and their names are placed on a notification list. This list is used when a renewal material is issued to notify customers of the price and availability of the item. Customers so notified are requested to submit a new order if they still want the item.

For some individually certified SRM's, production lots are small and may entail frequent stock outages. In these cases, the notification list is used to fill orders on a "first come, first served" basis. NOTE: For such SRM's, customers are notified that the SRM is again available and are requested to confirm their original purchase orders.

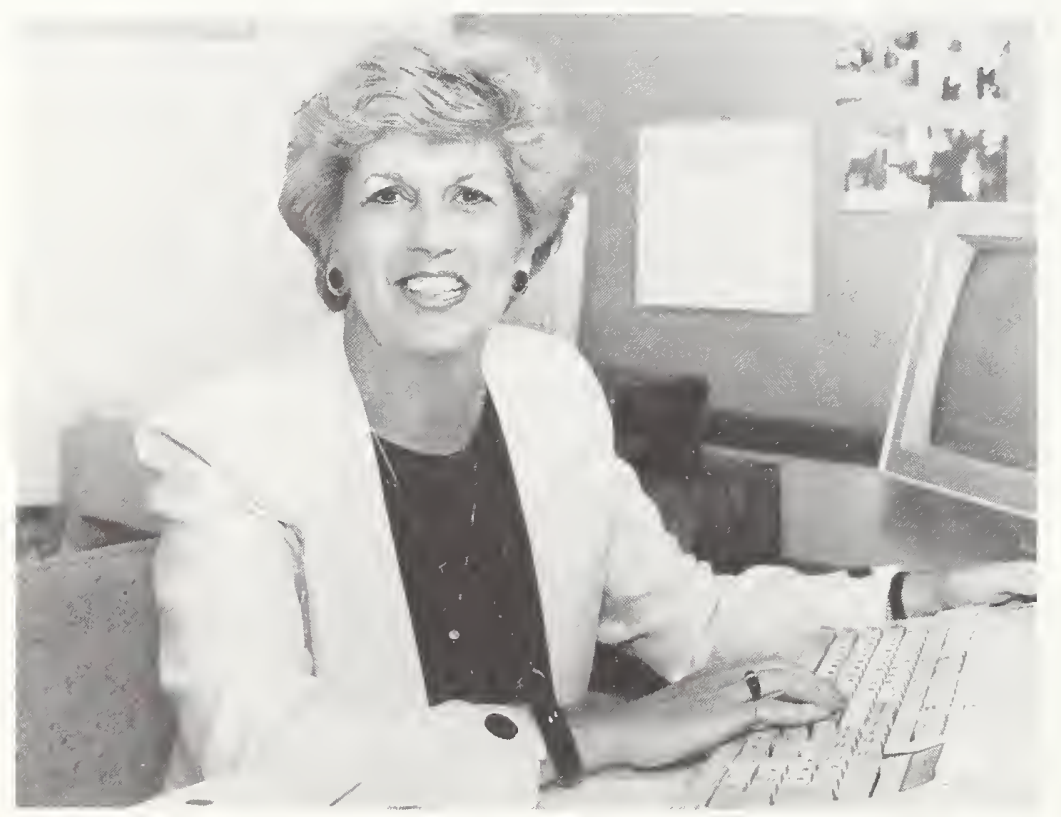

Lee Best, Manager of Sales and Marketing. has developed an excellent sales and marketing staff through the thoroughness and initiative of her leadership. We look forward to meeting many of you at one of the many exhibits Lee has scheduled for the Office of Standard Reference Materials. 


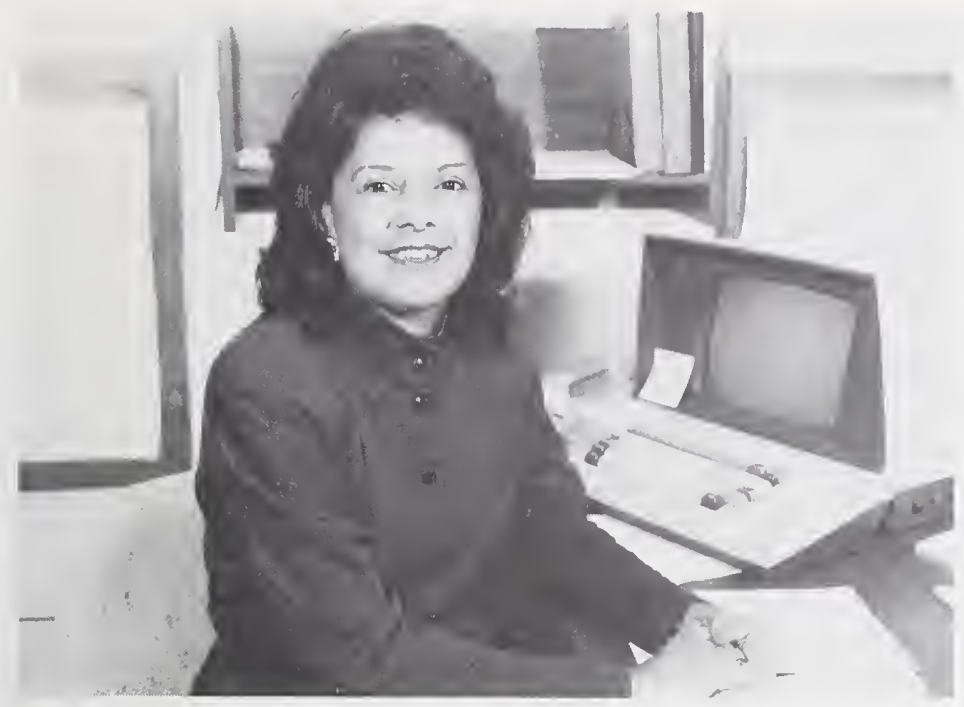

\section{Terms}

Prices quoted are in U.S. dollars (\$), and are published in the catalog supplements (price lists). When price lists are issued, they are sent to persons or organizations on the SRM mail list. These prices are subject to change without notice and orders will be billed for the prices in effect at the time of shipment. No discounts are given on purchases of SRM's or RM's.

Remittances of the purchase price need not accompany the purchase order. Payment of invoices is expected within 30 days of the receipt of the invoice. Payment on foreign orders may be made by any of the following:

a. Banker's draft against U.S.A. bank,

b. Bank to bank transfer to U.S.A. bank,

c. Cash against documents,

d. Sight draft,

e. International money order, or

f. UNESCO coupons.

Letters of credit: If a letter of credit or any method of payment other than those listed above is to be used, the services of an agent in the United States must be secured to act in your behalf. Your agent would purchase the material and our invoice would indicate that the agent is the purchaser. The material would be shipped to your agent, who would tranship in accordance with your instructions.

NOTE: A customer identification number; i.e., social security number (EIN) for consumer customers; tax identification number (TIN) for commercial customers; or agency location code (ALC) for government customers, is required with each order.

Phyllis Wagner, an SRM assistant, provides consistency and dependability to the delivery of many SRM's through her expertise in regulations and procedures for the shipment of hazardous materials.

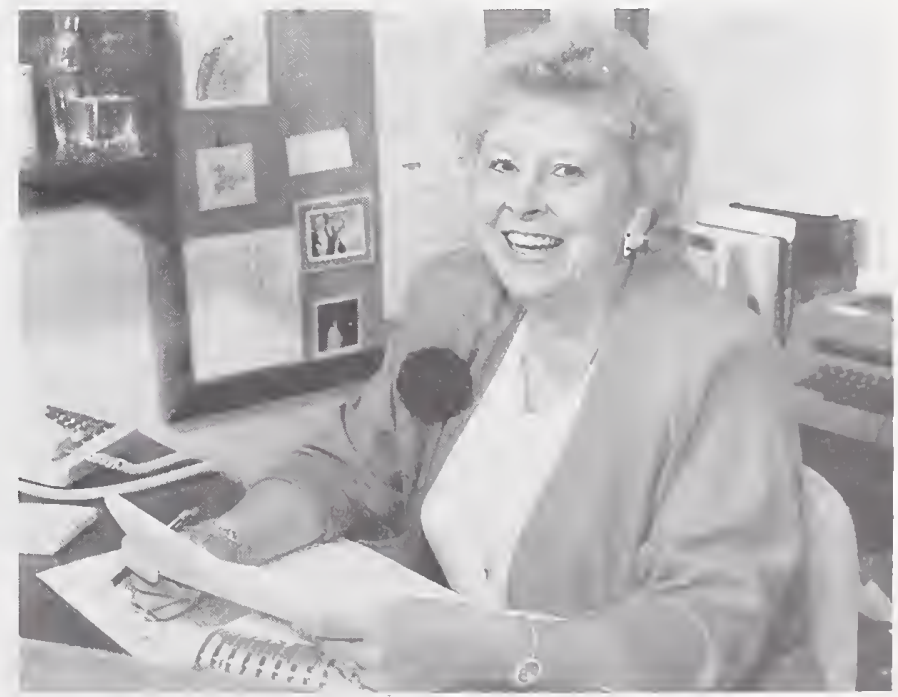




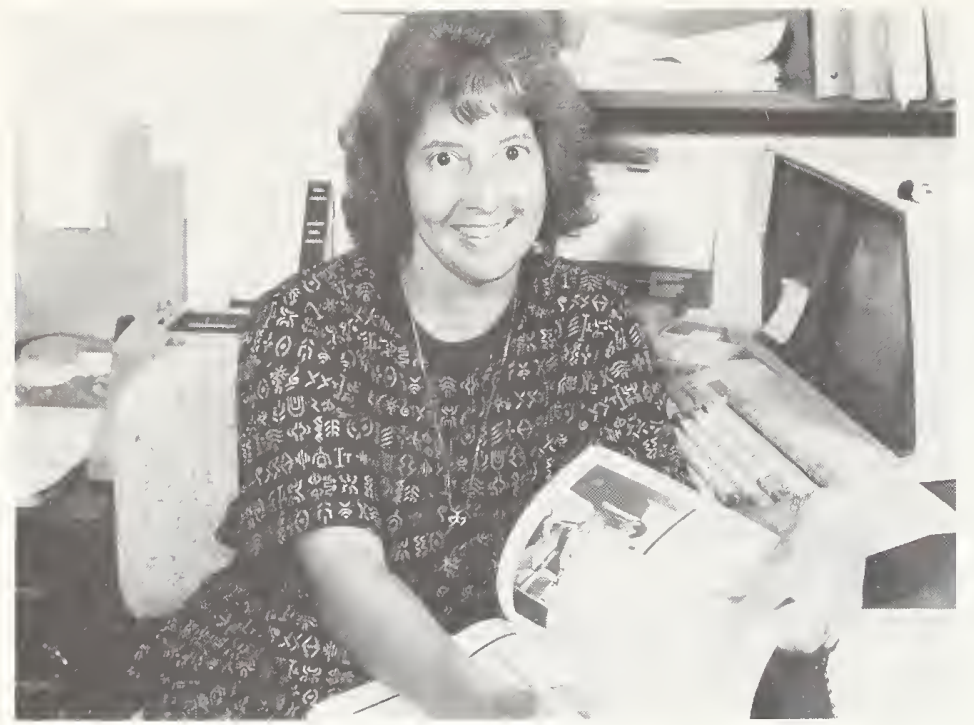

Cindy Leonard provides customer sales and services with a joyfulness and friendliness that is appreciated by our customers.

\section{Late Charges}

Unless otherwise notified, payment is due within 30 days of shipment of the order to the customer. U.S. Treasury regulations require that late charges be assessed for each 30-day period, or portion thereof, that the payment is overdue.

\section{Proforma Invoice (Price Quotation)}

Proforma service will be provided only to those requiring such service.

\section{Domestic Shipments}

Shipments of material (except for certain restricted categories and refrigerated items) intended for the United States and Canada are normally shipped prepaid, providing the parcel does not exceed the weight limitations prescribed by postal laws and regulations. Refrigerated items are shipped prepaid air express with shipping costs added to the invoice.

Donna Clark, systems analyst, displays persistence and attention to details in the development and maintenance of the OSRM computer programs for sales and inventory control.

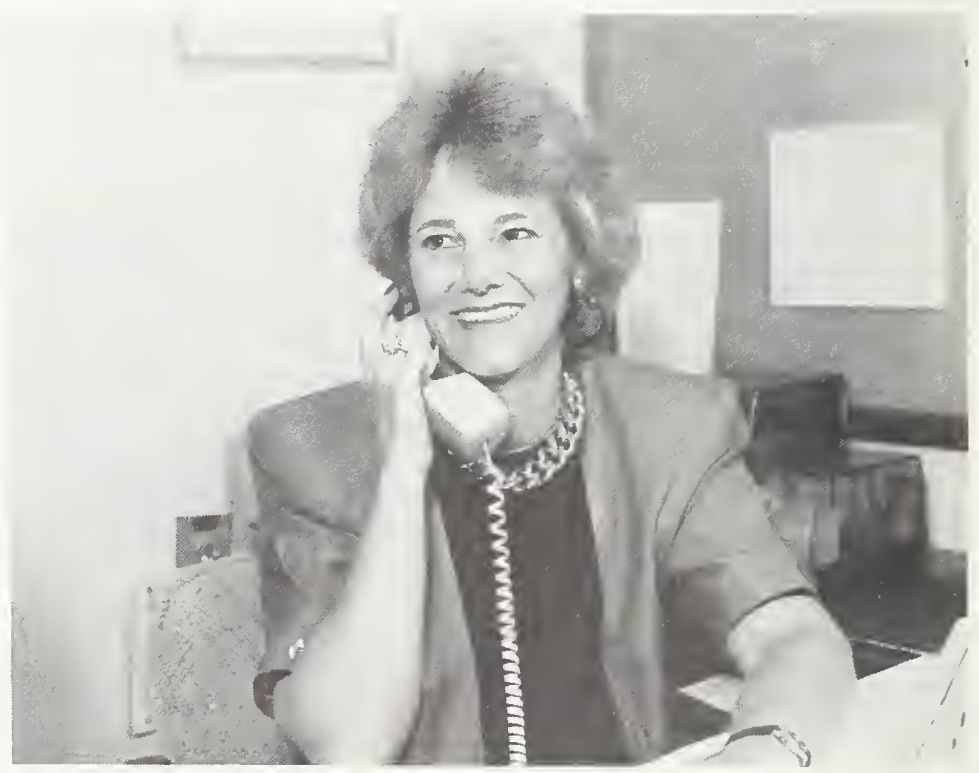




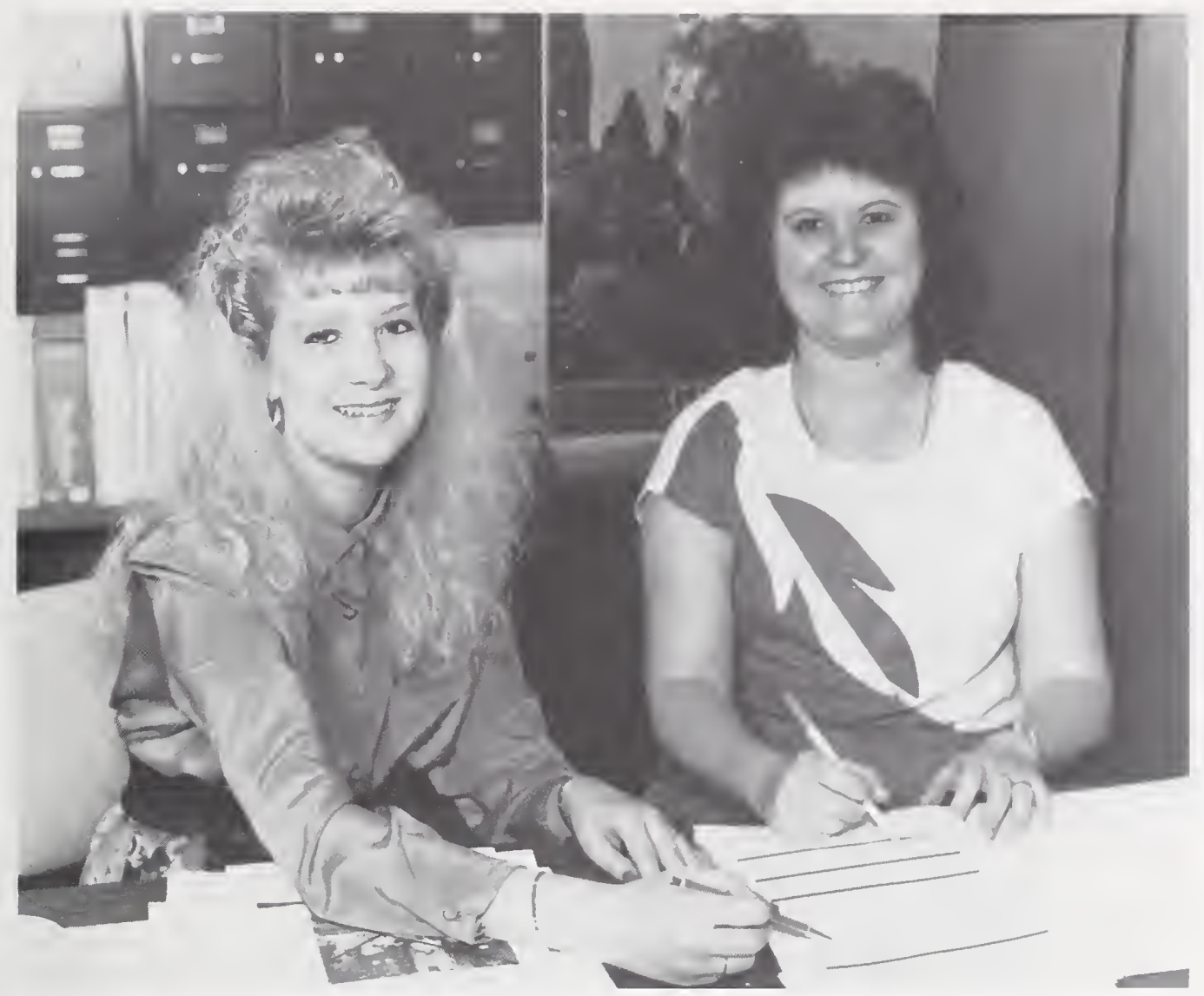

Jody Hines (lefi) and Sandy Barber combine their characteristic qualities of diligence, determination, flexibility and cooperativeness to the task of updating information for the preparation and publication of the new 1990.91 SRM Catalog.

\section{Foreign Shipments}

The regulations of various nations covering the importation of SRM's differ widely; any attempt to list all possible variations would be impractical. Therefore, where shipping practices outlined below do not apply, purchasers will be informed of the best method of shipment for their countries.

Most foreign orders will be shipped by prepaid International Air Parcel Post. Exceptions are those items in restricted categories, those items requiring refrigeration, and shipments exceeding parcel post weight limits. These exceptions will be shipped FOB Gaithersburg, MD, unless an agent (shipping or brokerage firm) located in the United States is used. When an agent is required, the purchaser will be notified and will be requested to obtain the services of one and inform us of the agent's name and address. In such cases, the material will be packed for overseas shipment and will be forwarded to the agent FOB Gaithersburg, MD.

\section{Documentation}

The documents we furnish are:
a. Two commercial invoices,
b. Two sight drafts,
c. Two packing slips, and
d. An air waybill for air shipments.

(All documents are printed in English.)

If documents other than those listed above are required, the services of an agent in the United States will be needed to purchase and ship the material.

NOTE: Orders and inquiries submitted in English will be processed more rapidly than those requiring translation. 


\section{Certified Reference Materials From Other Sources}

\section{Special Nuclear Materials}

On October 1, 1987, the New Brunswick Laboratory began issuing special nuclear reference materials as NBL Certified Reference Materials (CRM's). These CRM's include the plutonium and uranium assay and isotopic materials previously issued by the National Institute of Standards and Technology. All orders or inquiries should be addressed to:

U.S. Department of Energy

New Brunswick Laboratory

Attn: Reference Materials Sales

9800 S. Cass Avenue, Bldg. 350

Argonne, IL 60439

(312) $972-2767$

\section{International CRM's}

Certified reference materials (CRM's) are available from many sources. The International Organization for Standardization (ISO), through its Council Committee on Reference Materials (REMCO), has prepared an international Directory of Certified Reference Materials. Inquiries may be directed to:

Dr. M. Parkany

Secretary for REMCO

International Organization for Standardization

1 , Rue de Varembe

Case Postale 56

1211 Geneva 20

Switzerland

The International Union of Pure and Applied Chemistry (IUPAC), through its Commission on Physicochemical Measurements and Standards, issues a catalog of CRM's that are useful for the realization of physicochemical properties. It also has prepared a number of related documents. The current IUPAC edition is: "Physicochemical Measurements: Catalogue of Reference Materials from National Laboratories," Revised 1976, Pure \& Appl. Chem., 48, 503-515 (1976).

Julie Frum makes the Division Office a pleasant and productive place by her dependability and warm spirit of hospitality.

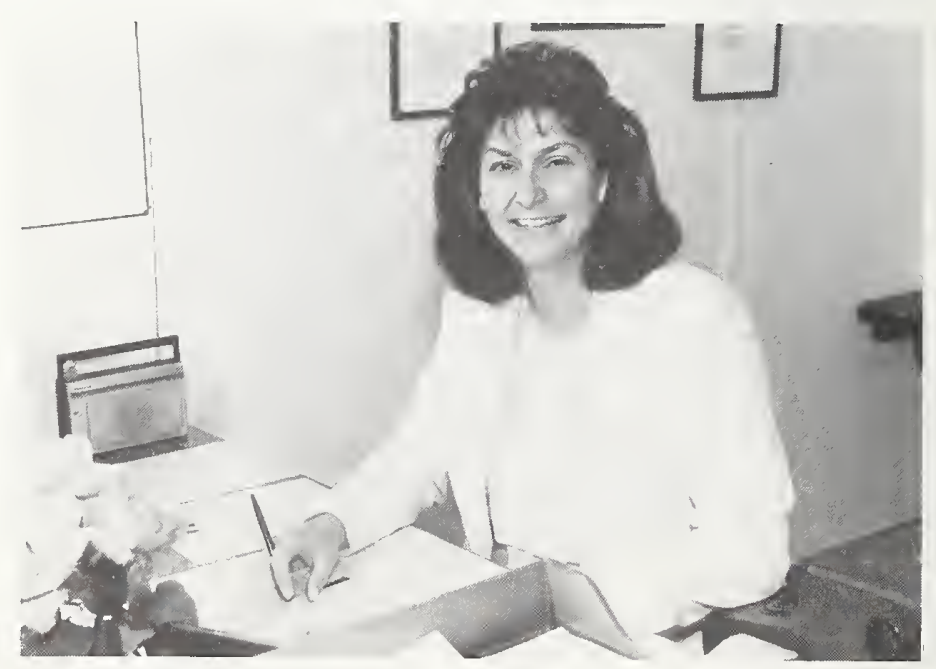

10 


\section{Other Services of the \\ National Institute of Standards and Technology}

\section{Calibration and Related Measurement Services}

The measurement services of NIST include the calibration of standards, test of instruments, and certain interlaboratory testing programs. These services are described in NIST Special Publication 250, National Institute of Standards and Technology Calibration Services Users Guide, 1989 ed. [Available from the Superintendent of Documents, U.S. Government Printing Office, Washington, DC 20402.]

An abbreviated list of the services offered through this program appears under Additional Information. These services are performed at either the NIST Washington laboratories (Gaithersburg, MD) or those in Boulder, CO. For additional information on available measurement services, consult Special Publication 250 or write to:

Office of Physical Measurement Services

Room B362 Physics Building

National Institute of Standards and Technology

Gaithersburg, MD 20899

Telephone: (301) 975-2002

Requests for measurement services available in Boulder should be addressed to:

Measurement Services Clerk

National Institute of Standards and Technology

Boulder, CO 80303

Telephone: (303) 497-3753

\section{Office of Weights and Measures}

The NIST Office of Weights and Measures operates a Type Evaluation Program which provides for an evaluation of (1) prototype weighing and measuring devices to determine compliance with the requirements of NBS Handbook 44, "Specifications, Tolerances, and Other Technical Requirements for Commercial Weighing and Measuring Devices," (2) standards to determine compliance with the requirements of NBS Handbook 105-1, 105-2, 105-3, "Specifications and Tolerances for Reference Standard and Field Standard Weights and Measures." This program may be used by manufacturers and weights and measures officials in determining the acceptability of devices for commercial use or the suitability of reference and field standards. For information on programs of NIST and the States, write or telephone:

Office of Weights and Measures

Room A617 Administration Building

National Institute of Standards and Technology

Gaithersburg, MD 20899

Telephone: (301) 975-4004 


\section{Proficiency Sample Programs}

General information on the Proficiency Sample Programs may be obtained from:

Materials Reference Laboratories

National Institute of Standards and Technology

Gaithersburg, MD 20899

Telephone: (301) 975-6704

Information is available on the following programs:

Proficiency Sample Programs for Hydraulic Cements and Portland Cement Concrete

Proficiency Sample Programs for Soils, Aggregates, and Bituminous Materials

Inspection of Cement and Concrete Testing Laboratories

Inspection of Soils and Bituminous Testing Laboratories

\section{Accreditation of Testing Laboratories}

General information about the National Voluntary Laboratory Accreditation Program (NVLAP) or application packages may be obtained from:

Manager, Laboratory Accreditation

Room A531 Administration Building

National Institute of Standards and Technology

Gaithersburg, MD 20899

Telephone: (301) 975-4016

\section{National Center for Standards and Certification Information}

The National Center for Standards and Certification Information (NCSCI) contains title information or full texts for more than 240,000 engineering or related standards issued by U.S. technical societies, professional organizations, and trade associations; State purchasing offices; U.S. Federal Government agencies; and major foreign national and international standardizing bodies. NCSCI publishes general and specific indices of standards. Information services which are free consist of searching Key-Word-In-Context (KWIC) Indices to determine whether any published standards, specifications, codes, test methods, or recommended practices exist for a given item or product. Inquiries should be directed to:

National Center for Standards and Certification Information

Room A629 Administration Building

National Institute of Standards and Technology

Gaithersburg, MD 20899

Telephone: (301) 975-4040

\section{National Standard Reference Data System}

The National Standard Reference Data System (NSRDS) is a nationwide program established to compile and critically evaluate quantitative physical science data and assure its availability to the technical community. The program publishes compilations of critically evaluated data, critical reviews of experimental techniques, and bibliographies. A complete list of NSRDS publications is available from the Office of Standard Reference Data (OSRD). OSRD responds to queries within the scope of the program by providing references, referrals, documentation, or data, as available. Inquiries or requests for information should be directed to:

Office of Standard Reference Data

Room A323 Physics Building

National Institute of Standards and Technology

Gaithersburg, MD 20899

Telephone: (301) 975-2208 


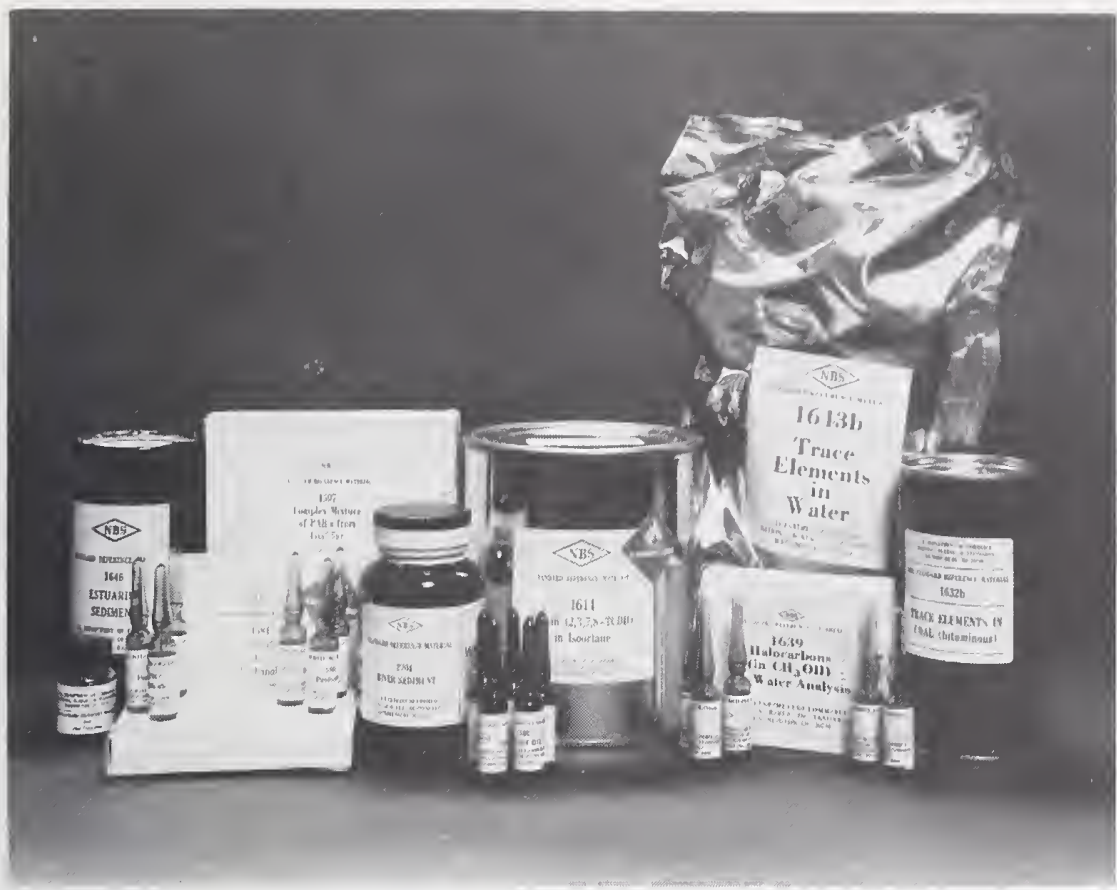

A sampling of Environmental SRM's which illustrate the diversity of SRM matrices and the creativity that must be exercised in the packaging of SRM's to maintain the integrity of the sample and its certified properties.

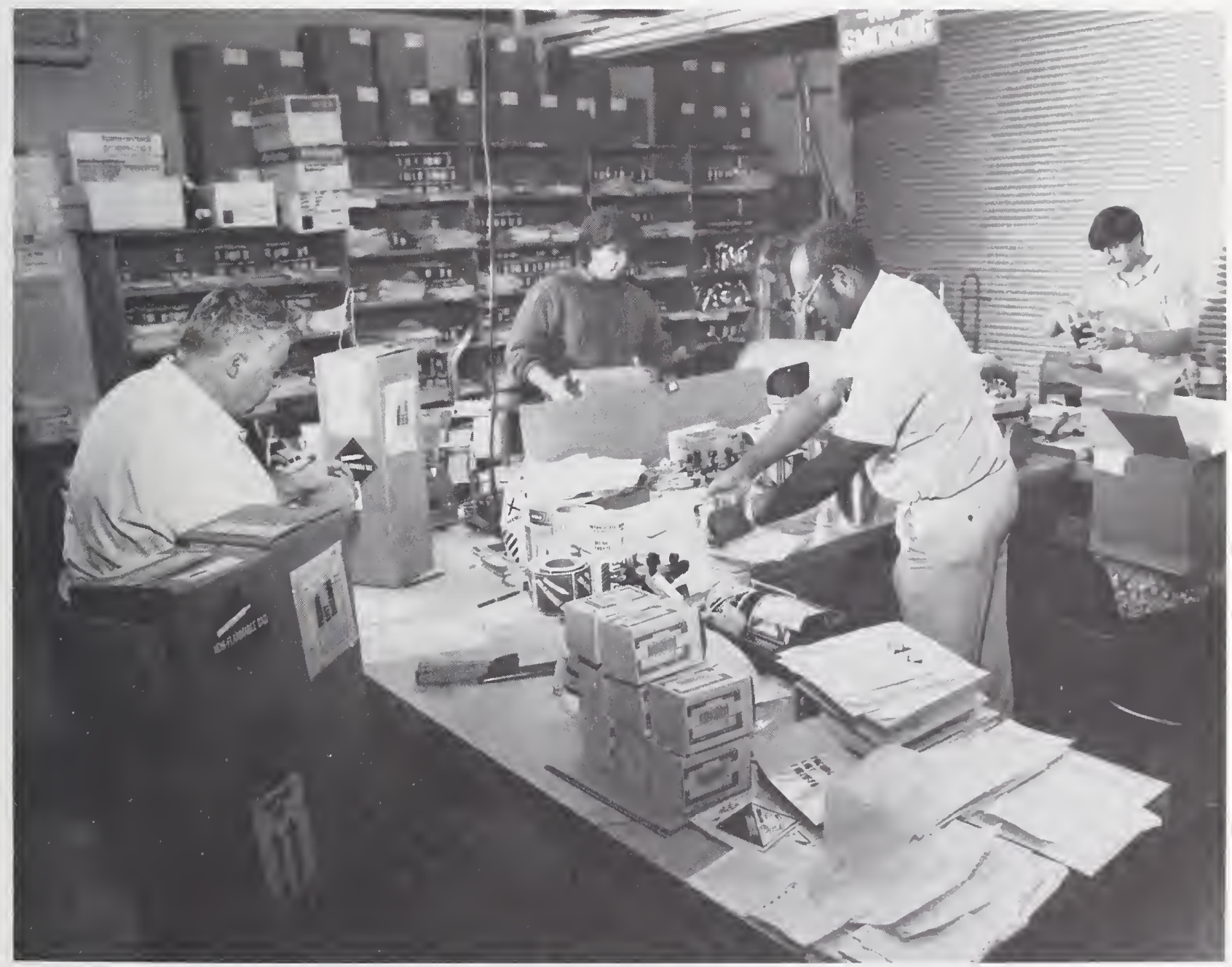

Roger Brown (lefi) contributes generous, industrious leadership to the shipping activities. Carlton Fisher (right front), whose qualities of orderliness and neatness combine with the responsibility and availability provided by Gary Proulx (center back) and Jim Fort (right back) to create a productive, efficient shipping department that sent out about 45,000 units this past year. 


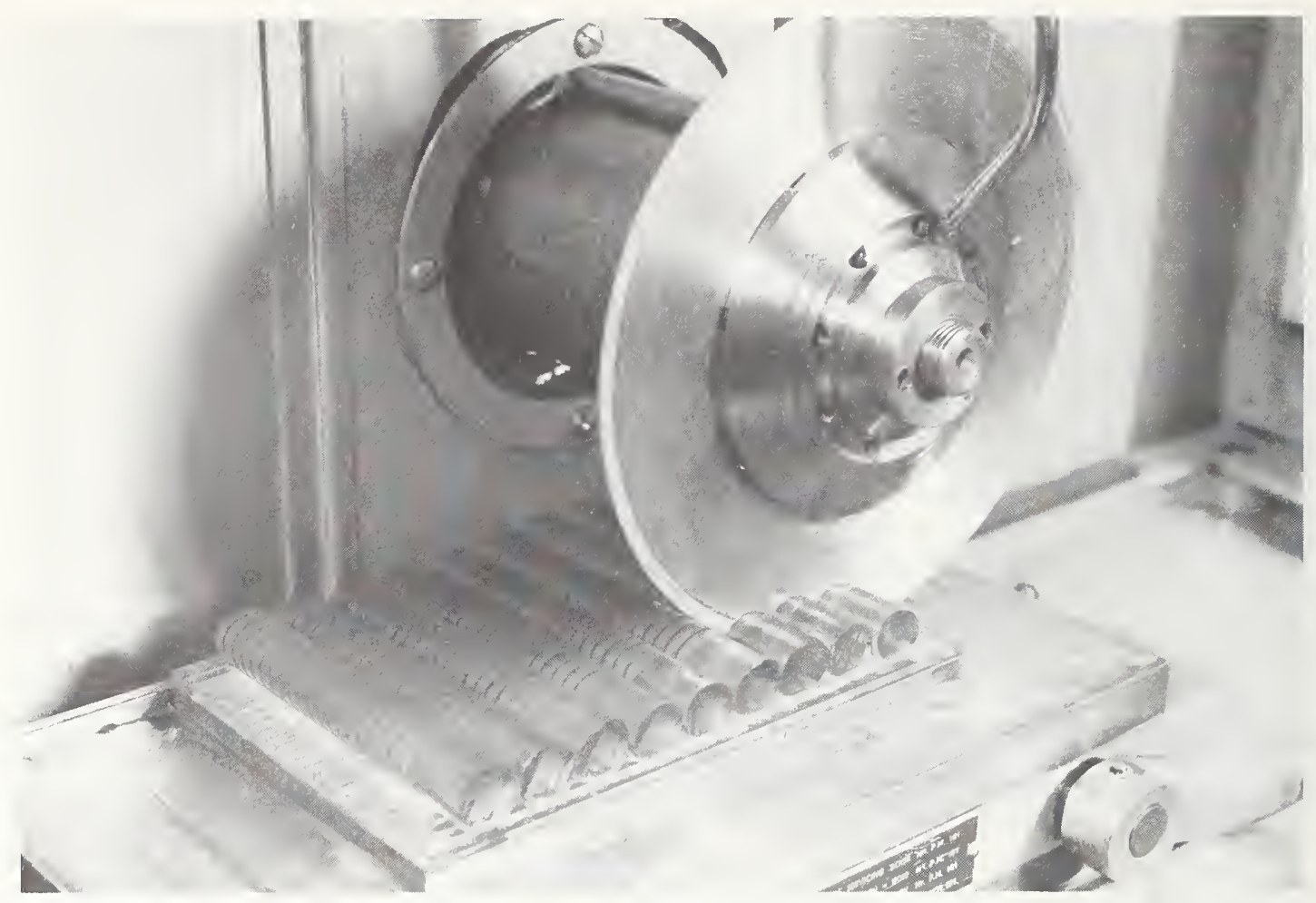

A diamond saw wafering machine, one of a wide variety of equipment used in the preparation of SRM materials, is being used to cut glass discs from glass rods.

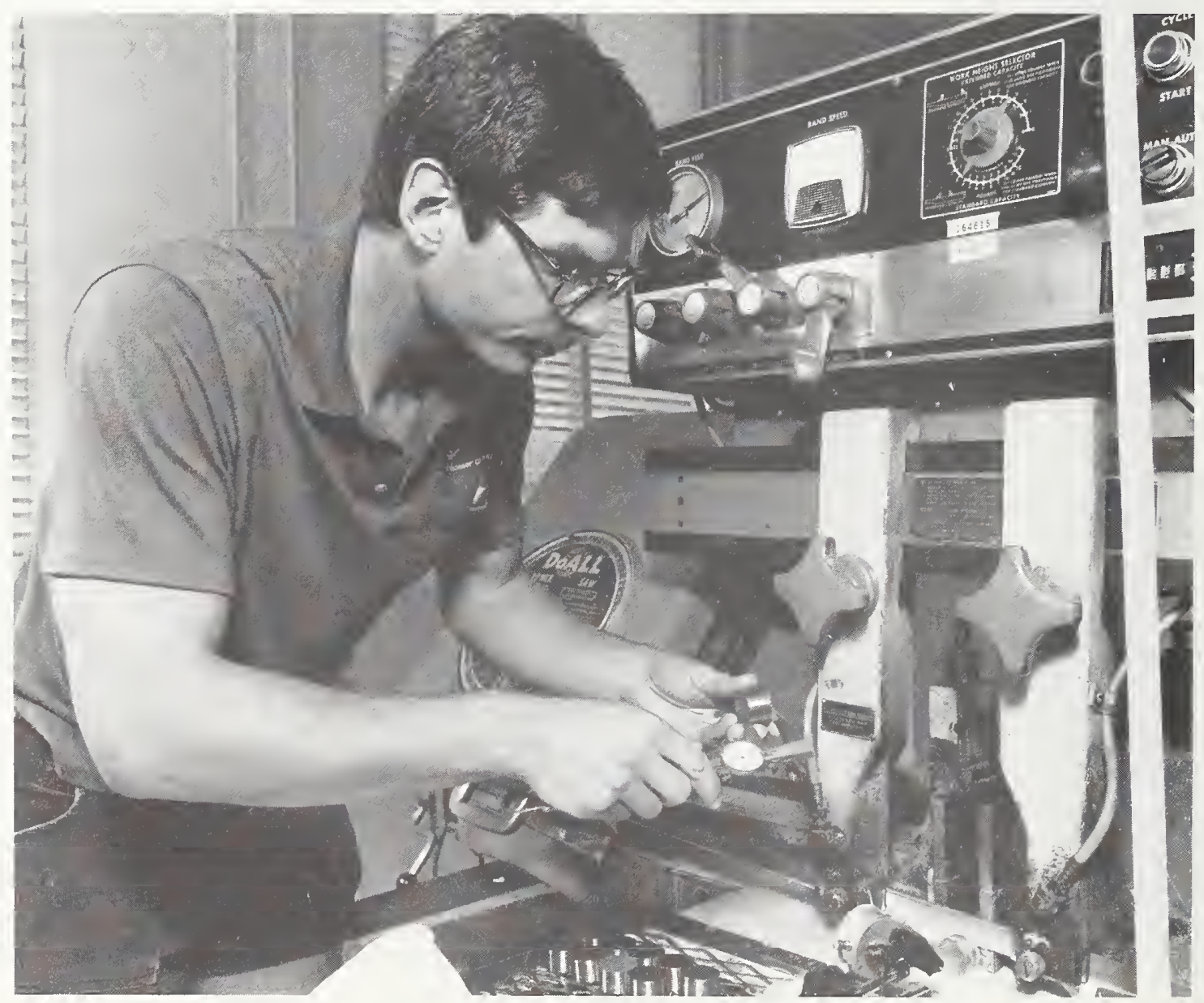

Dan Swearingen, shown here using an automated band saw to prepare samples for one of the metal SRM's, is appreciated for the dependability and diligence he applies to the activities of sample preparation. 


\section{Chemical Composition}

\section{Ferrous Alloys}

\section{Steels (Chip Form)}

These SRM's are for checking chemical methods of analysis. They consist of steel alloys selected to provide a wide range of analytical values for elements. They are furnished in 150-gram units (unless otherwise noted) as chips usually sized between 0.4 to $1.2 \mathrm{~mm}$, prepared from selected portions of commercial ingots.

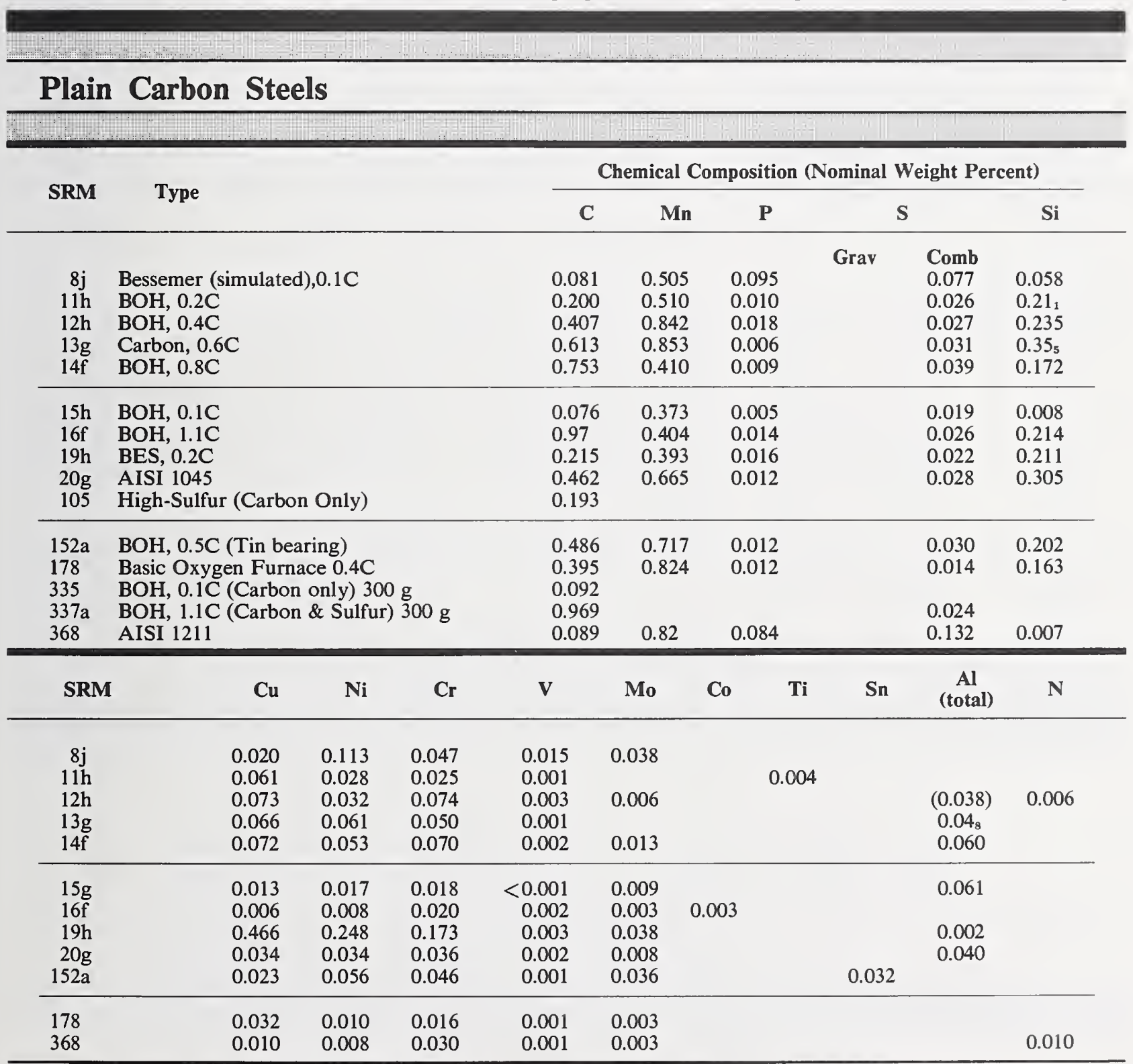

Values in parentheses are not certified, but are given for information only. 


\section{Low Alloy Steels}

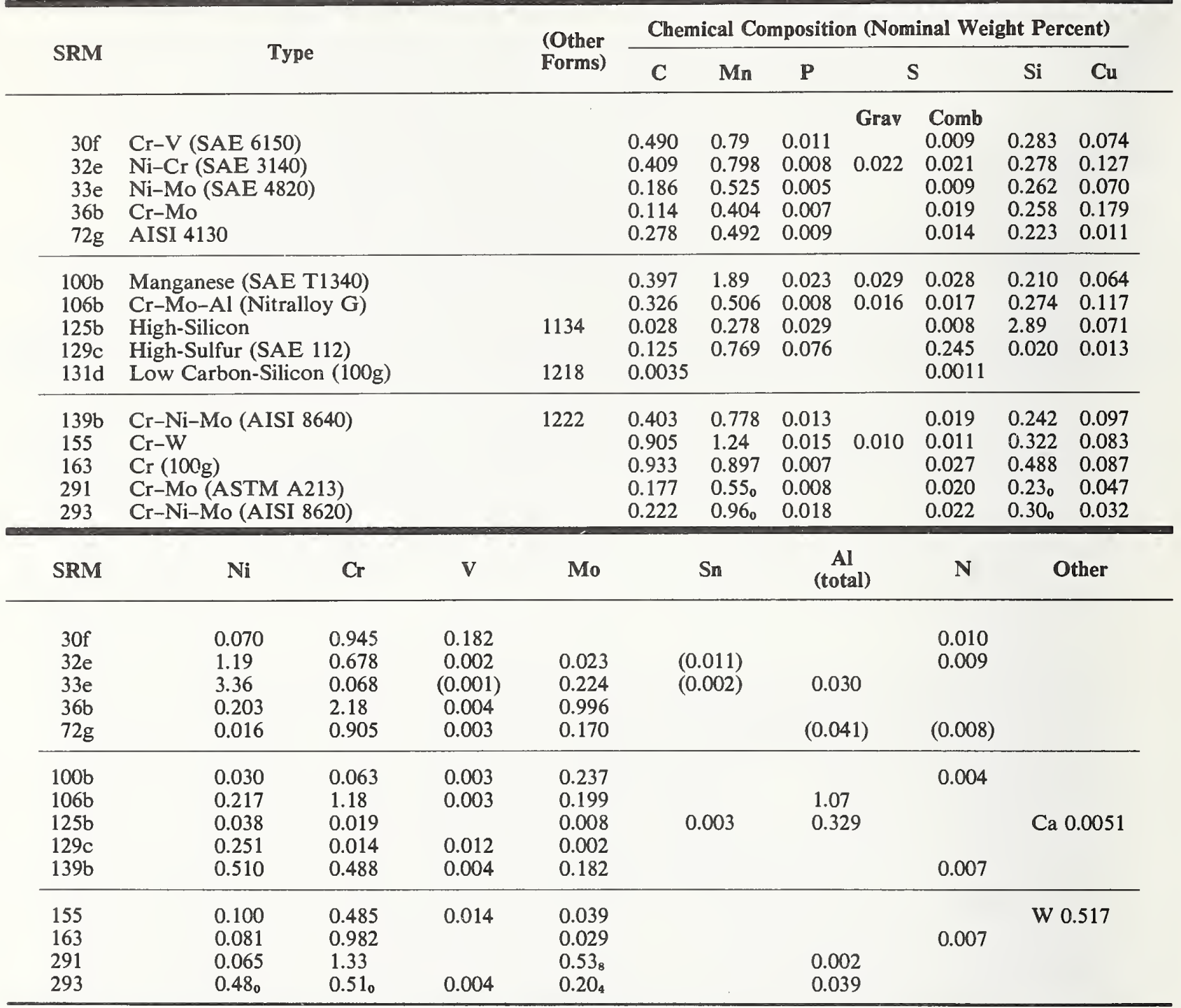

Values in parentheses are not certified, but are given for information only. 


\section{Special Low Alloy Steels}

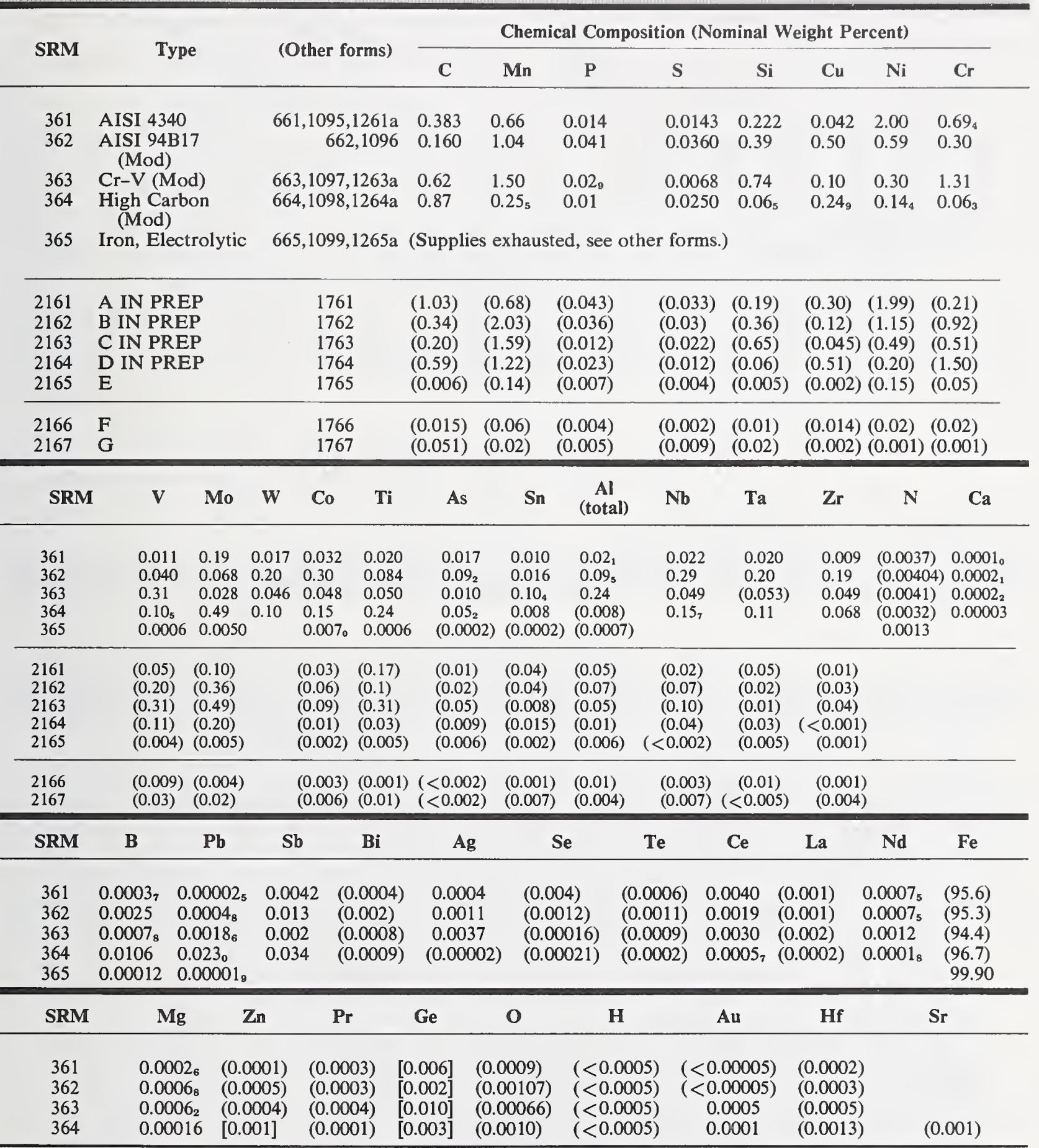

Values in parentheses are not certified, but are given for information only.

Values in brackets are approximate values from heat analysis and are given for information only. 


\section{High Alloy Steels}

\begin{tabular}{|c|c|c|c|c|c|c|c|c|c|c|c|c|}
\hline \multirow{2}{*}{ SRM } & \multirow{2}{*}{\multicolumn{3}{|c|}{ Type }} & & \multirow{2}{*}{$\begin{array}{c}\text { (Other } \\
\text { Forms) }\end{array}$} & \multicolumn{7}{|c|}{ Chemical Composition (Nominal Weight Percent) } \\
\hline & & & & & & $\mathrm{C}$ & Mn & $\mathbf{P}$ & \multicolumn{2}{|c|}{$\mathbf{S}$} & \multirow[t]{2}{*}{$\mathrm{Si}$} & \multirow[t]{2}{*}{$\mathrm{Cu}$} \\
\hline & & & & & & & & & Grav & Comb & & \\
\hline $126 c$ & High-Nicl & $\operatorname{kel}(36 \% \mathrm{I}$ & $\mathrm{Ni}$ ). & & 1158 & 0.025 & 0.468 & \multirow{2}{*}{$\begin{array}{l}0.004 \\
0.018\end{array}$} & & 0.005 & 0.194 & 0.040 \\
\hline 344 & $\begin{array}{l}\mathrm{Cr}-\mathrm{Ni} \text { ( } \mathrm{M} \\
\text { ing) }\end{array}$ & Io Precipi & itation & Harden- & & 0.069 & 0.57 & & & & 0.395 & 0.106 \\
\hline 345 & $\begin{array}{c}\mathrm{Cr}-\mathrm{Ni} \\
\text { ing) }\end{array}$ & u Precipit & itation 1 & Harden- & & 0.048 & 0.224 & 0.018 & 0.012 & 0.012 & 0.610 & 3.44 \\
\hline $346 a$ & Valve Ste & & & & 1233 & 0.502 & 9.16 & $\begin{array}{l}0.031 \\
0.023\end{array}$ & & 0.002 & 0.219 & 0.375 \\
\hline $348 a$ & $\begin{array}{l}\text { High } \mathrm{Ter} \\
\mathrm{Ni}-\mathrm{Cr}\end{array}$ & nperature & e Alloy & (A286) & 1230 & 0.044 & 0.64 & 023 & & 0.0007 & 0.43 & 0.14 \\
\hline 868 & $\begin{array}{c}\text { High } \\
\text { Co }\end{array}$ & mperature & e Alloy & $\mathrm{Fe}-\mathrm{Ni}-$ & 1250 & 0.022 & $0.052<0$ & $<0.003$ & & 0.0025 & 0.097 & 0.022 \\
\hline SRM & $\mathbf{N i}$ & $\mathrm{Cr}$ & $\mathbf{V}$ & Mo & Co & Ti & \multicolumn{2}{|l|}{ Al (total) } & \multicolumn{2}{|c|}{ Ta } & B & $\mathbf{F e}$ \\
\hline $126 c$ & 36.05 & $0.062 \quad 0$ & 0.001 & 0.011 & 0.008 & & & & & & & \\
\hline 344 & 7.28 & 14.95 & 0.040 & 2.40 & & 0.076 & 1.16 & & & & & \\
\hline 345 & 4.24 & 16.04 & 0.041 & 0.122 & 0.089 & & & 0.231 & & 0.002 & & \\
\hline $346 a$ & 3.43 & 21.08 & 0.096 & 0.237 & $(0.05)$ & $(<0.001)$ & $(0.001)$ & $(0.01)$ & Sn $(C$ & $0.008)$ & $<0.001)$ & N 0.415 \\
\hline $348 a$ & 24.2 & 14.8 & 0.23 & 1.18 & 0.15 & 2.12 & 0.24 & $(0.07)$ & W (C & $0.07)$ & 0.0055 & $(55.2)$ \\
\hline 868 & 37.78 & $0.077 \quad 0$ & 0.077 & 0.014 & 16.1 & 1.48 & 0.99 & 2.99 & & 0.003 & 0.0078 & 40.5 \\
\hline
\end{tabular}

Values in parentheses are not certified, but are given for information only.

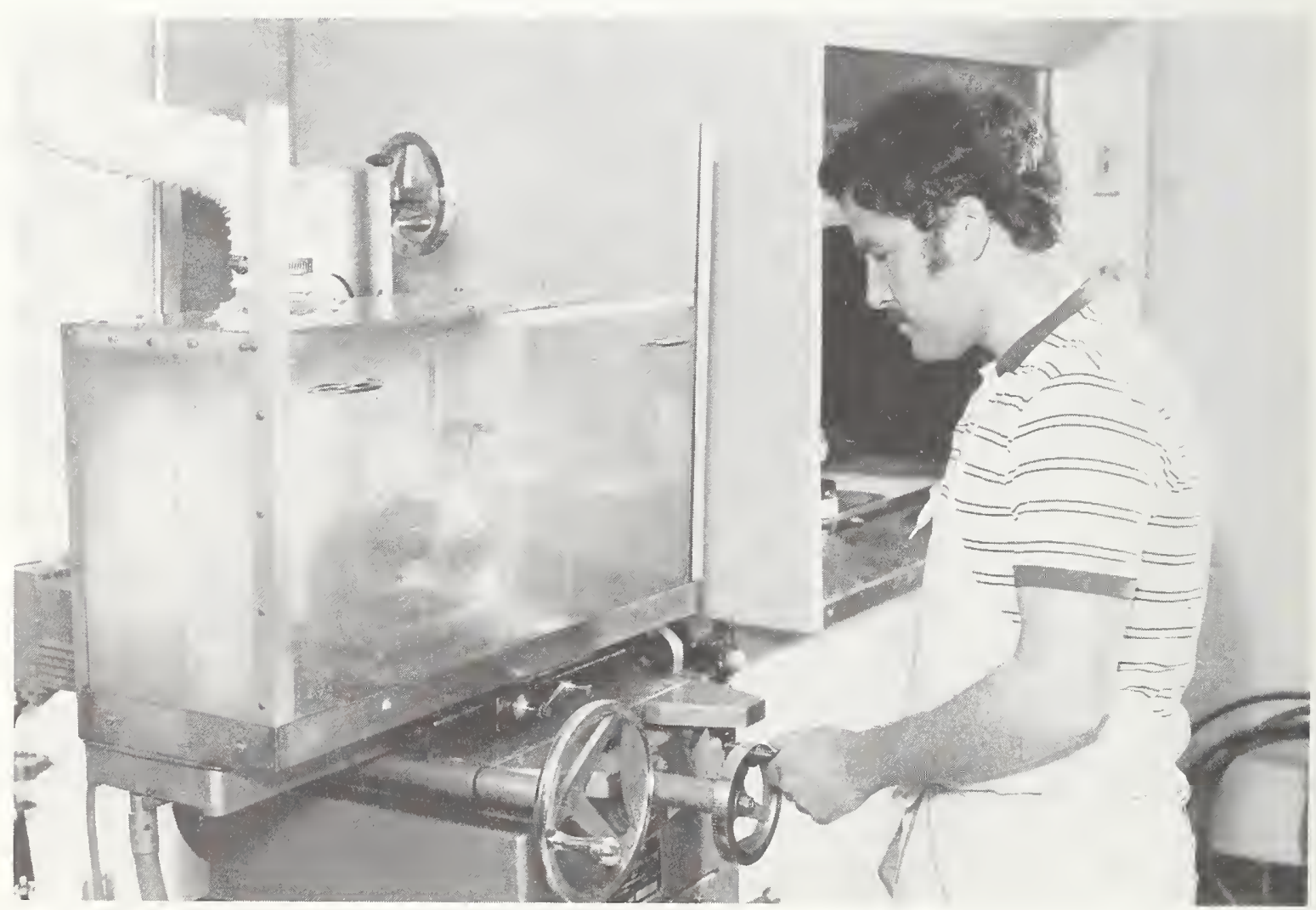

Curt Fales, a valuable member of the materials preparation staff because of his resourcefulness and attention to detail, is shown here using the diamond saw wafering machine. 
Stainless Steels

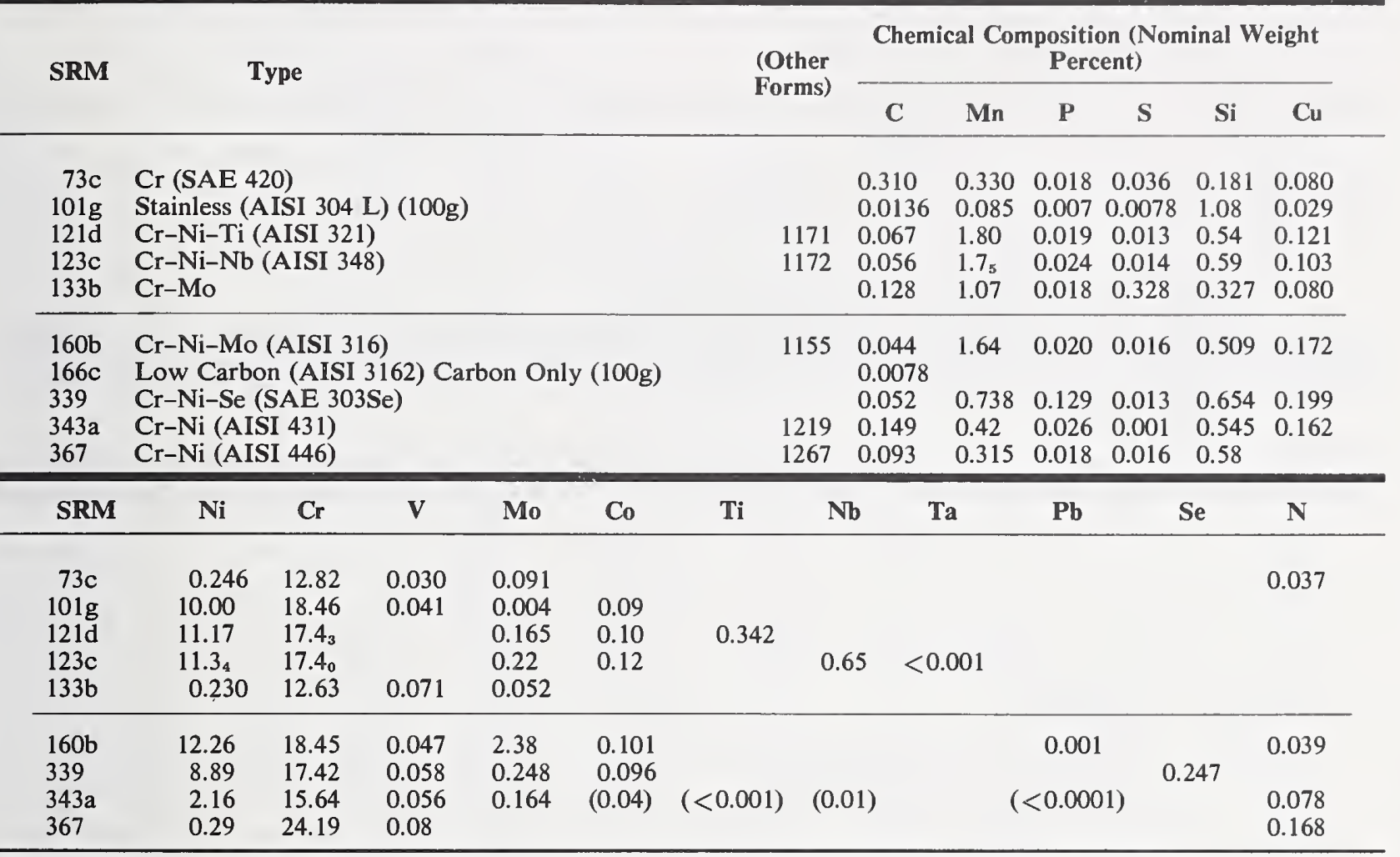

Values in parentheses are not certified, but are given for information only.

\section{Tool Steels}

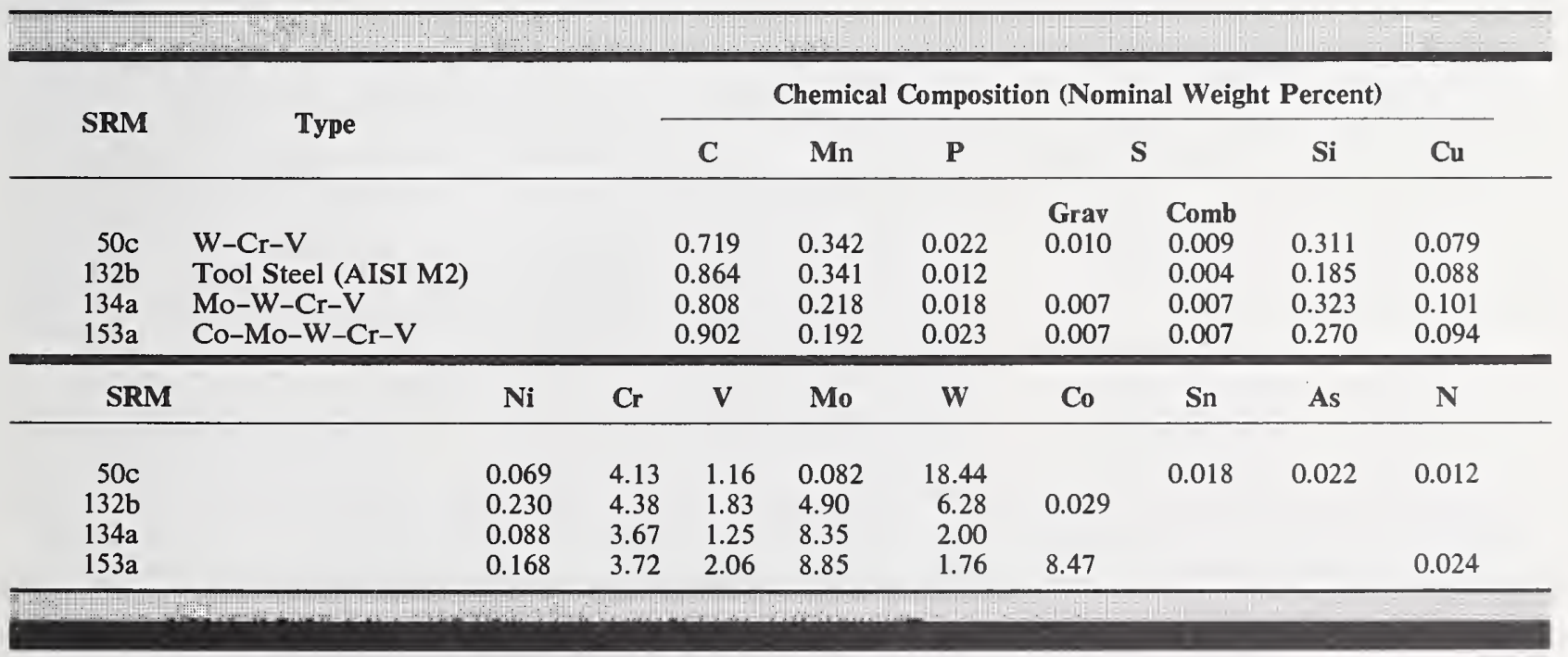




\section{Steels (Solid Form)}

These SRM's are furnished in various forms. The 600 series is for microchemical methods of analysis such as electron probe microanalysis, spark source mass spectrometric analysis, and laser probe analysis. The 1100 , 1200, and 1700 series are for optical emission and x-ray spectroscopic methods of analysis. These materials have been prepared to ensure high homogeneity.

NOTE: Values in parentheses are not certified, but are given for additional information on the chemical composition.

\section{Nominal Sizes for Solid Steel SRM's:}

600 Series: $3.2 \mathrm{~mm}(1 / 8 \mathrm{in})$ diameter, $51 \mathrm{~mm}(2 \mathrm{in})$ long.

1100,1200 , and 1700 Series: $31 \mathrm{~mm}\left(1 \frac{1 / 4}{\mathrm{in}}\right)$ diameter, $19 \mathrm{~mm}(3 / 4 \mathrm{in})$ thick.

$\mathrm{C}$ indicates a chill cast sample: $31 \mathrm{~mm}\left(1 \frac{1 / 4}{\mathrm{in}}\right)$ diameter, $19 \mathrm{~mm}(3 / 4 \mathrm{in})$ thick.

\section{Low-Alloy Steels}

\begin{tabular}{|c|c|c|c|c|c|c|c|}
\hline \multirow{2}{*}{ SRM } & \multirow{2}{*}{ Type } & \multirow{2}{*}{$\begin{array}{l}\text { (Other } \\
\text { Forms) }\end{array}$} & \multicolumn{5}{|c|}{$\begin{array}{c}\text { Chemical Composition (Nominal Weight } \\
\text { Percent) }\end{array}$} \\
\hline & & & C & Mn & $\mathbf{P}$ & $\mathbf{S}$ & $\mathbf{S i}$ \\
\hline 1134 & High-Silicon & $125 b$ & 0.026 & 0.277 & 0.028 & 0.009 & 2.89 \\
\hline 1135 & High-Silicon & 179 & 0.027 & 0.094 & 0.006 & 0.026 & 3.19 \\
\hline 1217 & Nickel & $33 \mathrm{e}$ & 0.186 & 0.525 & 0.005 & 0.009 & 0.262 \\
\hline 1218 & Low Carbon and Sulfur Silicon & $131 d$ & 0.0029 & 0.014 & $(0.002)$ & 0.0011 & $(3.2)$ \\
\hline C1221 & $\begin{array}{l}\text { Resulfurized/Rephosphorized, } \\
\text { (AISI 1211) }\end{array}$ & & 0.020 & 0.102 & 0.090 & 0.112 & 0.876 \\
\hline 1222 & Cr-Ni-Mo (AISI 8640) & $139 b$ & 0.43 & 0.78 & 0.013 & 0.022 & 0.24 \\
\hline 1224 & Carbon (AISI 4130) & & 0.75 & 0.41 & 0.009 & 0.039 & 0.173 \\
\hline 1225 & Low Alloy (AISI 4130) & & 0.274 & 0.48 & 0.007 & 0.014 & 0.221 \\
\hline 1226 & Low Alloy & & 0.085 & 0.274 & 0.0022 & 0.0044 & 0.231 \\
\hline 1227 & Basic Open Hearth, $1 \% \mathrm{C}$ & & 0.97 & 0.402 & 0.014 & 0.026 & 0.215 \\
\hline 1228 & Basic Open Hearth, $0.1 \% \mathrm{C}$ & & 0.072 & 0.365 & 0.004 & 0.018 & 0.007 \\
\hline 1254 & Ca in Low Alloy (Si) & & $(0.03)$ & $(0.28)$ & $(0.03)$ & $(0.008)$ & $(2.9)$ \\
\hline$* 661$ & AISI 4340 & & $0.39_{2}$ & 0.66 & 0.015 & 0.015 & 0.223 \\
\hline *662 & AISI 94B17 (Modified) & & 0.163 & 1.05 & 0.044 & 0.037 & 0.40 \\
\hline$* 663$ & $\mathrm{Cr}-\mathrm{V}$ (Modified) & & 0.57 & 1.50 & 0.029 & 0.0055 & 0.74 \\
\hline *664 & High Carbon (Modified) & & $0.87_{1}$ & $0.25_{s}$ & 0.010 & 0.025 & 0.066 \\
\hline *665 & Electrolytic Iron & & 0.008 & 0.0057 & $0.002_{5}$ & 0.0059 & 0.008 。 \\
\hline 1269 & Line Pipe Steel (AISI 1526, Mod) & & 0.298 & 1.35 & 0.012 & 0.0061 & 0.189 \\
\hline 1270 & Cr-Mo Low Alloy, A336 (F-22) & & 0.077 & 0.626 & 0.0065 & 0.0065 & 0.247 \\
\hline C1285 & Low Alloy (A242 Mod) & & 0.058 & 0.332 & 0.072 & 0.020 & 0.36 \\
\hline 1286 & Low Alloy (Hy 80) & & 0.196 & 0.152 & 0.008 & 0.017 & 0.130 \\
\hline 1761 & Low Alloy Steel & 2161 & 1.03 & 0.678 & 0.042 & 0.035 & 0.18 \\
\hline 1762 & Low Alloy Steel & 2162 & 0.337 & 2.00 & 0.034 & 0.030 & 0.35 \\
\hline 1763 & Low Alloy Steel & 2163 & 0.203 & 1.58 & 0.012 & 0.023 & 0.63 \\
\hline 1764 & Low Alloy Steel & 2164 & 0.592 & 1.21 & 0.021 & 0.012 & 0.057 \\
\hline 1765 & Low Alloy Steel & 2165 & 0.006 & 0.144 & 0.006 & 0.0038 & 0.004 \\
\hline 1766 & Low Alloy Steel & 2166 & 0.015 & 0.067 & 0.002 & 0.0024 & 0.010 \\
\hline 1767 & Low Alloy Steel & 2167 & 0.052 & 0.021 & 0.004 & 0.0090 & 0.026 \\
\hline
\end{tabular}

Values in parentheses are not certified, but are given for information only.

*SRM's 661, 662, 663, 664, and 665 are sold in a set only as SRM 668. 


\section{Low-Alloy Steels (Continued)}

\begin{tabular}{ccccccccc}
\hline SRM & Cu & Ni & Cr & V & Mo & W & Co & Ti \\
\hline 1134 & 0.070 & 0.038 & 0.019 & & 0.008 & & & \\
1135 & 0.056 & 0.050 & 0.022 & $<0.01$ & 0.014 & & & \\
1217 & 0.070 & 3.36 & 0.068 & $(0.001)$ & 0.224 & & $(0.06)$ & $(0.001)$ \\
1218 & 0.003 & $(0.002)$ & 0.006 & $(<0.001)$ & $(0.003)$ & & $(0.002)$ & $(0.004)$ \\
C1221 & 0.041 & 0.067 & 0.049 & $(0.0007)$ & 0.038 & & $(0.010)$ & $(0.0014)$ \\
\hline 1222 & 0.097 & 0.51 & 0.48 & 0.005 & 0.18 & & $(0.016)$ & $(0.002)$ \\
1224 & 0.072 & 0.054 & 0.071 & 0.002 & 0.013 & & & \\
1225 & & 0.018 & 0.91 & 0.004 & 0.166 & & & \\
1226 & 0.125 & 5.42 & 0.467 & 0.0018 & 0.446 & $(0.005)$ & 0.029 & 0.0021 \\
1227 & 0.006 & 0.007 & 0.019 & 0.002 & 0.003 & & 0.003 & $(0.0008)$ \\
\hline 1228 & 0.012 & 0.018 & 0.016 & $<0.001$ & 0.009 & & & \\
1254 & $(0.07)$ & $(0.04)$ & $(0.02)$ & & $(0.008)$ & & & \\
$* 661$ & 0.042 & 1.99 & 0.69 & 0.011 & 0.19 & 0.017 & 0.032 & 0.020 \\
$* 662$ & 0.51 & 0.60 & 0.30 & 0.04, & 0.07 & 0.21 & 0.30 & 0.084 \\
$* 663$ & 0.098 & 0.32 & 1.31 & 0.31 & 0.030 & 0.04 & 0.048 & 0.050 \\
\hline$* 664$ & 0.250 & 0.142 & $0.06_{6}$ & $0.10_{6}$ & 0.49 & $0.10_{2}$ & 0.15 & 0.23 \\
$* 665$ & 0.0058 & 0.041 & $0.007_{2}$ & 0.0006 & 0.0050 & $<1$ & 0.007 & 0.0006 \\
1269 & 0.095 & 0.108 & 0.201 & 0.004 & 0.036 & $(0.001)$ & $(0.014)$ & $(0.009)$ \\
1270 & 0.114 & 0.174 & 2.34 & 0.013 & 0.956 & $(0.003)$ & 0.038 & $(0.003)$ \\
C1285 & 0.37 & 1.17 & 0.80 & 0.150 & 0.164 & $(0.03)$ & 0.036 & Ce $(0.0021)$ \\
\hline 1286 & 0.043 & 2.81 & 1.53 & 0.0057 & 0.334 & $(0.13)$ & 0.116 & 0.040 \\
1761 & 0.30 & 1.99 & 0.220 & 0.053 & 0.103 & $(0.02)$ & $(0.028)$ & 0.18 \\
1762 & 0.120 & 1.15 & 0.92 & 0.200 & 0.35 & $(0.01)$ & 0.062 & 0.095 \\
1763 & 0.43 & 0.51 & 0.50 & 0.30 & 0.50 & $(0.03)$ & 0.095 & 0.31 \\
1764 & 0.51 & 0.202 & 1.48 & 0.106 & 0.200 & $(<0.01)$ & $(0.01)$ & 0.028 \\
\hline 1765 & $(0.0015)$ & 0.154 & 0.051 & 0.0040 & 0.005 & $(0.001)$ & $(0.001)$ & 0.0055 \\
1766 & 0.015 & 0.021 & 0.024 & 0.009 & 0.0035 & $(0.001)$ & 0.0020 & 0.0005 \\
1767 & 0.0014 & 0.002 & 0.002 & 0.033 & 0.021 & $(0.003)$ & 0.005 & 0.011 \\
\hline
\end{tabular}

Values in parentheses are not certified, but are given for information only.

*SRM's $661,662,663,664$, and 665 are sold in a set only as SRM 668.

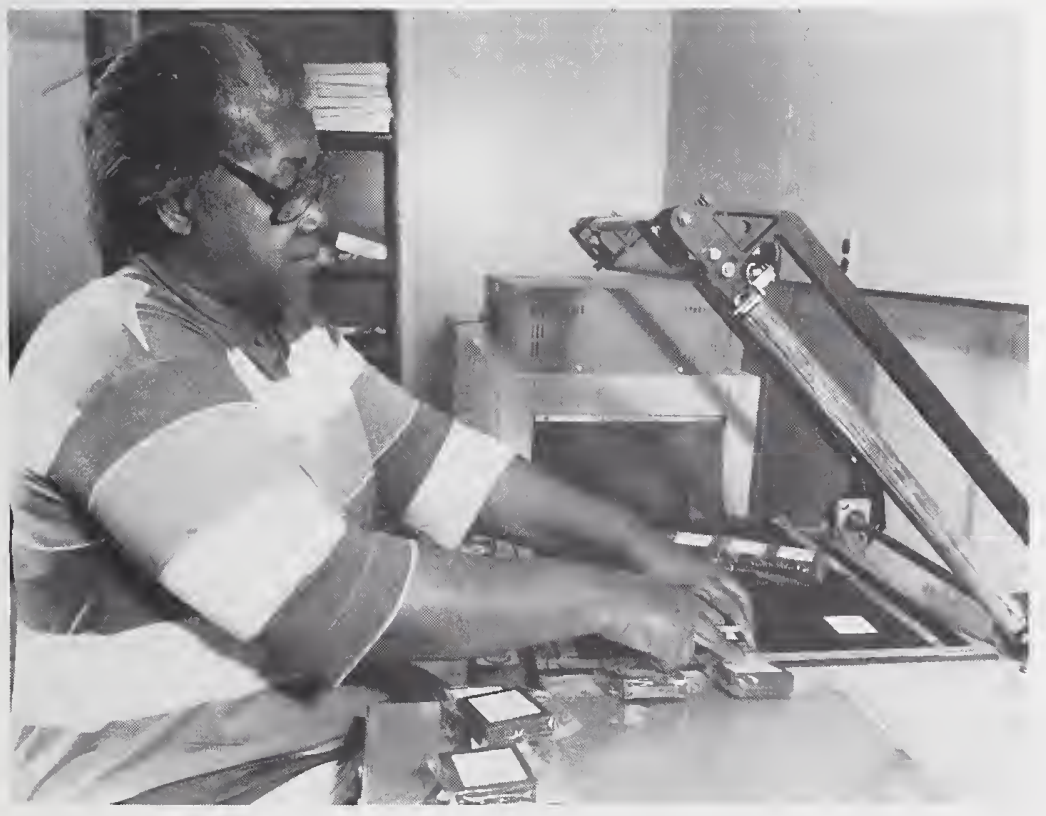

Helen Tyler, shown here applying heat-shrink packaging, ensures the integrity of packaging activities through her dependability and alertness to the details of packaging many different materials. 


\section{Low-Alloy Steels (Continued)}

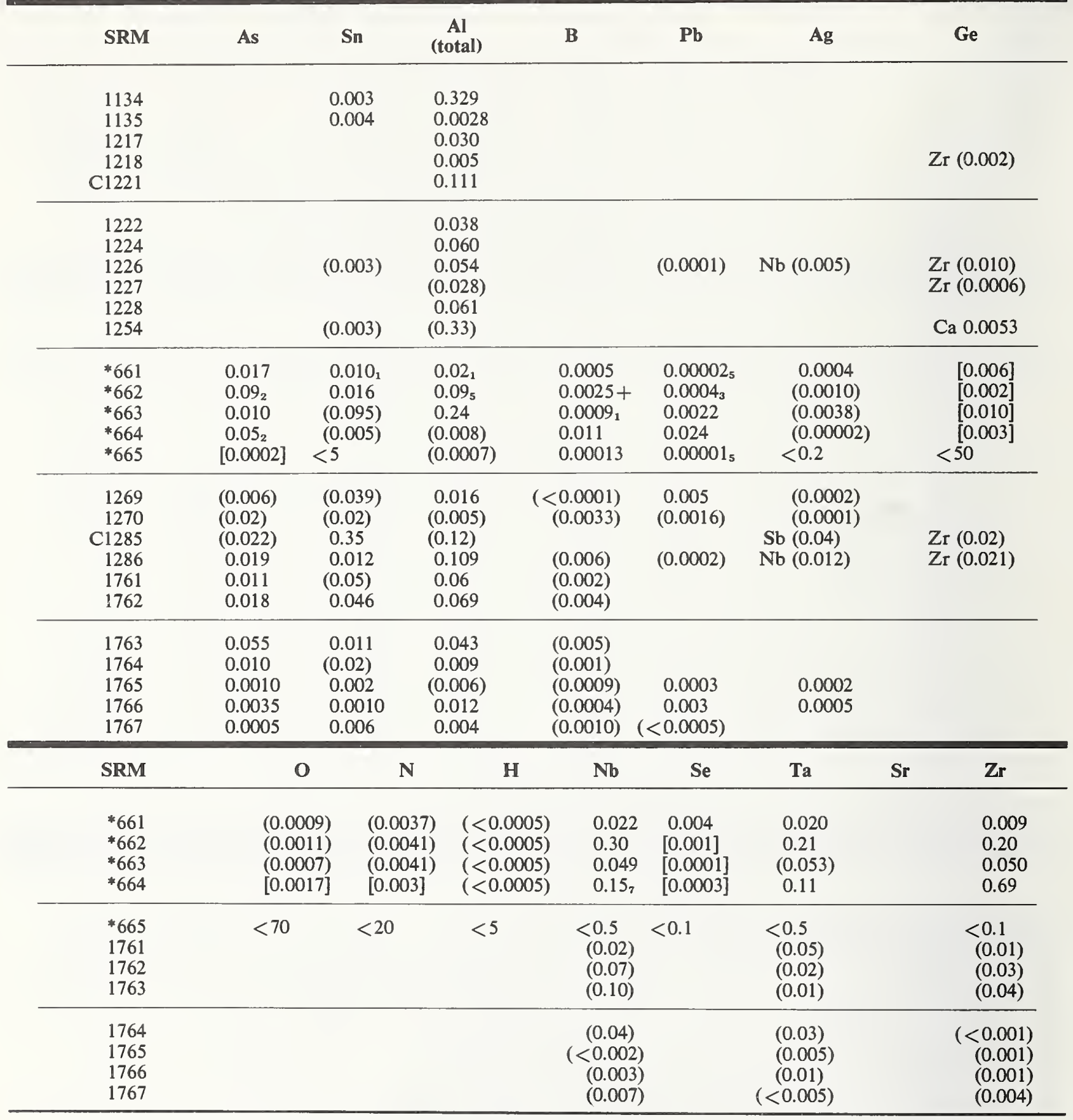

*SRM's 661, 662, 663, 664, and 665 are sold in a set only as SRM 668.

Values in parentheses are not certified, but are given for information only.

Values in brackets are approximate values from heat analysis and are given for information only. 


\section{Low-Alloy Steels (Continued)}

\begin{tabular}{|c|c|c|c|c|c|c|c|}
\hline SRM & $\mathbf{S b}$ & $\mathbf{B i}$ & $\mathrm{Ca}$ & \multicolumn{2}{|l|}{$\mathbf{M g}$} & \multicolumn{2}{|r|}{$\mathrm{Zn}$} \\
\hline $\begin{array}{l}* 661 \\
* 662 \\
* 663 \\
* 664 \\
* 665\end{array}$ & $\begin{array}{l}0.004_{2} \\
0.012 \\
(0.0007) \\
(0.035) \\
<0.5\end{array}$ & $\begin{array}{l}(0.002) \\
(0.0008) \\
(0.0009) \\
<0.5\end{array}$ & $\begin{array}{r}(<0.0001) \\
(0.0002) \\
(<0.0001) \\
(<0.0001) \\
<0.1\end{array}$ & \multicolumn{2}{|c|}{$\begin{array}{l}(0.0001) \\
(0.0006) \\
(0.0005) \\
(0.0001) \\
<0.2\end{array}$} & \multicolumn{2}{|c|}{$\begin{array}{l}(0.0001) \\
(0.0005) \\
(0.0004) \\
{[0.001]} \\
<3\end{array}$} \\
\hline SRM & Au & $\mathrm{Ce}$ & Hf & $\mathbf{L a}$ & Nd & $\operatorname{Pr}$ & $\mathrm{Fe}$ \\
\hline $\begin{array}{l}* 661 \\
* 662 \\
* 663 \\
* 664 \\
* 665\end{array}$ & $\begin{array}{c}(<0.0005) \\
(<0.00005) \\
0.0005 \\
0.0001 \\
<0.02\end{array}$ & $\begin{array}{l}0.001_{3} \\
(0.0011) \\
(0.0016) \\
<0.05\end{array}$ & $\begin{array}{l}{[0.0002]} \\
{[0.006]} \\
{[0.0015]} \\
{[0.005]} \\
<0.2\end{array}$ & $\begin{array}{l}0.0004 \\
0.0004 \\
0.0006 \\
0.00007 \\
<0.05\end{array}$ & $\begin{array}{c}0.0003 \\
(0.0005) \\
(0.0007) \\
(0.00012) \\
<0.05\end{array}$ & $\begin{array}{l}(0.00014) \\
(0.00012) \\
(0.00018) \\
(0.00003) \\
<0.05\end{array}$ & $\begin{array}{ll}\text { 4) } & (95.6) \\
\text { 2) } & (95.3) \\
\text { 3) } & (94.4) \\
\text { 3) } & (96.7) \\
& <99.9\end{array}$ \\
\hline
\end{tabular}

*SRM's 661, 662, 663, 664, and 665 are sold in a set only as SRM 668.

Values in parentheses are not certified, but are given for information only.

Values in brackets are approximate values from heat analysis and are given for information only.

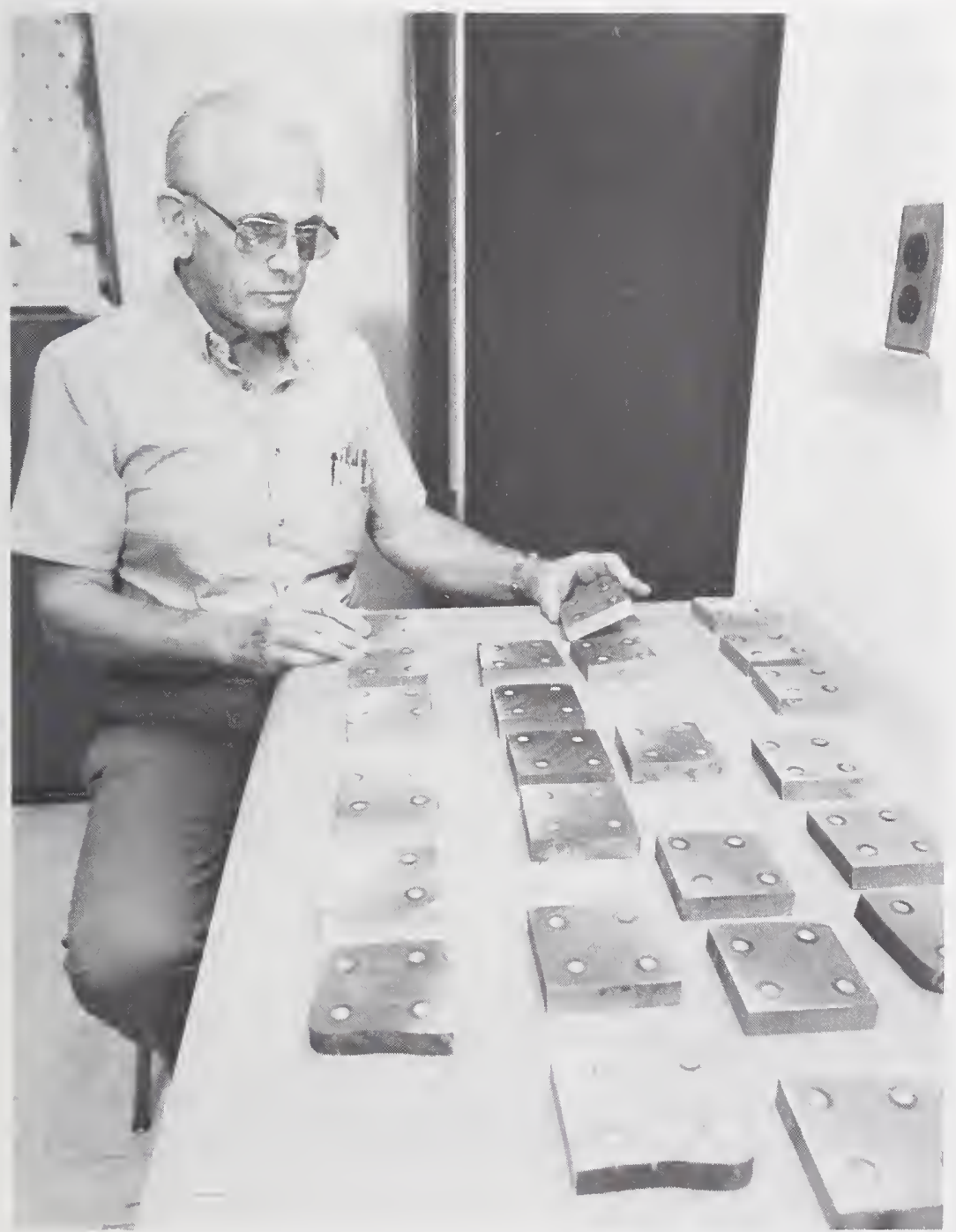

John Norris applies the knowledge and skill of many years experience to the inspection of steel samples that have been analyzed by spark discharge. 


\section{Stainless Steels}

\begin{tabular}{|c|c|c|c|c|c|c|c|c|c|c|c|c|c|}
\hline \multirow{2}{*}{ SRM } & \multirow{2}{*}{\multicolumn{4}{|c|}{ Type }} & \multirow{2}{*}{$\begin{array}{l}\text { Other } \\
\text { Forms }\end{array}$} & \multicolumn{8}{|c|}{ Chemical Composition (Nominal Weight Percent) } \\
\hline & & & & & & C & Mn & $\mathbf{P}$ & S & $\mathrm{Si}$ & $\mathbf{C u}$ & Ni & $\mathrm{Cr}$ \\
\hline C1151 & $\mathrm{Cr}-\mathrm{Ni}$ & & & & & 0.039 & 2.50 & 0.017 & 0.038 & 0.38 & 0.418 & 7.29 & 22.70 \\
\hline C1152 & $\mathrm{Cr}-\mathrm{Ni}$ & & & & & 0.148 & 0.96 & 0.021 & 0.0064 & 0.80 & 0.1021 & 10.88 & 17.81 \\
\hline C1153 & $\mathrm{Cr}-\mathrm{Ni}$ & & & & & 0.264 & 0.50 & 0.030 & 0.018 & 1.07 & 0.23 & 8.77 & 16.69 \\
\hline C1153a & $\mathrm{Cr}-\mathrm{Ni}$ & & & & & 0.225 & 0.544 & $0.03 C$ & 0.019 & 1.00 & 0.226 & 8.76 & 16.70 \\
\hline C1154 & $\mathrm{Cr}-\mathrm{Ni}$ & & & & & 0.086 & 1.42 & 0.06 & 0.053 & 0.50 & $0.40 \quad 1$ & 12.92 & 19.06 \\
\hline 1155 & \multirow{5}{*}{\multicolumn{4}{|c|}{$\begin{array}{l}\text { Cr-Ni-Mo (AISI 316) } \\
\text { Cr-Ni-Ti } \\
\text { Cr-Ni-Nb } \\
\text { Cr-Ni (AISI 431) } \\
\text { Chromium Steel }\end{array}$}} & $160 \mathrm{~b}$ & 0.046 & 1.63 & 0.020 & 0.018 & $0.50_{2}$ & $0.169 \quad 1$ & $12.1_{8}$ & $18.4_{5}$ \\
\hline 1171 & & & & & $121 d$ & 0.067 & 1.80 & $0.01 \varepsilon$ & $0.01_{3}$ & 0.54 & $0.121 \quad 1$ & 11.2 & 17.4 \\
\hline 1172 & & & & & $123 \mathrm{c}$ & 0.056 & $1.7_{6}$ & 0.02 & $0.01_{4}$ & 0.59 & $\begin{array}{ll}0.10_{5} & 1\end{array}$ & $11.3_{5}$ & $17.4_{0}$ \\
\hline 1219 & & & & & $343 a$ & 0.149 & 0.42 & 0.026 & 0.001 & 0.545 & 0.162 & 2.16 & 15.64 \\
\hline 1223 & & & & & $133 b$ & 0.127 & 1.08 & 0.018 & 0.329 & 0.327 & 0.081 & 0.232 & 12.64 \\
\hline 1267 & \multicolumn{4}{|c|}{ AISI 446} & 367 & 0.093 & \multirow{2}{*}{$\begin{array}{l}0.315 \\
1.66\end{array}$} & \multirow{2}{*}{$\begin{array}{l}0.018 \\
0.029\end{array}$} & 0.015 & 0.58 & & 0.29 & 24.14 \\
\hline C1287 & \multicolumn{4}{|c|}{$\begin{array}{l}\text { High-Alloy, ACI HK (AISI } \\
\text { 310, Mod.) }\end{array}$} & & 0.36 & & & 0.024 & 1.66 & 0.58 & 21.16 & 23.98 \\
\hline C1288 & \multicolumn{4}{|c|}{$\begin{array}{l}\text { High-Alloy, ACI CN-7M (A- } \\
\text { 743) }\end{array}$} & & 0.056 & 0.83 & 0.023 & 0.010 & 0.41 & 3.72 & 29.3 & 19.55 \\
\hline C1289 & \multicolumn{4}{|c|}{$\begin{array}{l}\text { High-Alloy, ACI CA-6NM } \\
\text { (AISI } 414 \text { Mod.) }\end{array}$} & & 0.014 & 0.35 & 0.017 & 0.021 & 0.156 & 0.205 & 4.13 & 12.12 \\
\hline SRM & v & Mo & Co & $\mathbf{T i}$ & $\mathbf{N}$ & Al & \multicolumn{2}{|c|}{$\mathrm{Nb}$} & $\mathrm{Ta}$ & $\mathbf{W}$ & $\mathbf{P b}$ & & $\mathrm{Zr}$ \\
\hline $\mathrm{C} 1151$ & 0.037 & 0.80 & & $(0.006)$ & $(0.23)$ & $(0.004)$ & \multirow{4}{*}{\multicolumn{2}{|c|}{$\begin{array}{l}(0.014) \\
(0.16) \\
(0.050) \\
(0.48)\end{array}$}} & \multirow{4}{*}{$\begin{array}{l}(0.006) \\
(0.001) \\
(0.032) \\
(0.03)\end{array}$} & & 0.0039 & \multirow{4}{*}{\multicolumn{2}{|c|}{$\begin{array}{l}(0.005) \\
(0.004) \\
(0.003) \\
(0.0001)\end{array}$}} \\
\hline C1152 & 0.030 & 0.43 & 0.22 & $(0.011)$ & $(0.055)$ & $(0.004)$ & & & & & 0.0047 & & \\
\hline C1153 & 0.18 & & 0.127 & $(0.014)$ & $(0.134)$ & $(0.003)$ & & & & & 0.0054 & & \\
\hline $\mathrm{C} 1153 \mathrm{a}$ & 0.176 & 0.24 & 0.127 & $(0.013)$ & $(0.11)$ & $(0.004)$ & & & & & 0.006 & & \\
\hline \multirow{5}{*}{$\begin{array}{r}\mathrm{C} 1154 \\
1155 \\
1171 \\
1172 \\
1219\end{array}$} & & 0.07 & 0.38 & $(0.004)^{\circ}$ & $(0.084)$ & $(0.004)$ & $(0.2$ & & $(0.075)$ & & 0.0178 & $(0.00$ & 04) \\
\hline & 0.047 & 2.38 & $0.10_{1}$ & & & & & & & & 0.001 & & \\
\hline & & $0.16_{5}$ & 0.10 & 0.34 & & & & & & & & & \\
\hline & & 0.22 & 0.12 & & & & 0.6 & & $<0.001$ & & & & \\
\hline & 0.056 & 0.164 & $(0.04)$ & $(<0.001)$ & 0.078 & $(0.001)$ & $\left(0 . c^{2}\right.$ & & Sn $(0.008)$ & $(0.02)$ & $(<0.0001)$ & B $(<$ & $<0.001)$ \\
\hline $\begin{array}{l}1223 \\
1267\end{array}$ & $\begin{array}{l}0.068 \\
0.08\end{array}$ & 0.053 & & & $(0.05)$ & $(<0.005)$ & & & Sn (0.004) & & $(0.0001)$ & & \\
\hline $\mathrm{C} 1287$ & $\begin{array}{l}0.08 \\
0.09\end{array}$ & 0.46 & 0.31 & 0.050 & $(0.034)$ & $(0.06)$ & (0.C & & $\mathrm{O}(0.017)$ & & 0.008 & $(0.00$ & 06) \\
\hline C1288 & 0.086 & 2.83 & 0.10 & 0.012 & $(0.028)$ & $(0.0025$ & 5) $(0.2$ & 2) & $\mathrm{O}(0.029)$ & $(0.2)$ & 0.0041 & $(0.00$ & 02) \\
\hline C1289 & 0.007 & 0.82 & 0.035 & 0.005 & $(0.017)$ & $(0.0016$ & 6) $(0.1$ & & $\mathrm{O}(0.027)$ & & 0.0005 & $(0.00$ & D1) \\
\hline
\end{tabular}

Values in parentheses are not certified, but are given for information only.

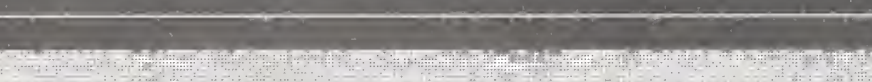

\section{Specialty Steels}

\begin{tabular}{|c|c|c|c|c|c|c|c|c|c|c|c|c|c|}
\hline \multirow{2}{*}{ SRM } & \multirow{2}{*}{ Type } & \multicolumn{12}{|c|}{ Chemical Composition (Nominal Weight Percent) } \\
\hline & & C & $\mathbf{M n}$ & $\mathbf{P}$ & $\mathbf{S}$ & Si & $\mathrm{Cu}$ & $\mathrm{Ni}$ & $\mathrm{Cr}$ & $\mathbf{V}$ & Mo & $\mathbf{w}$ & Co \\
\hline 1157 & Tool (AISI M2) & 0.836 & 0.34 & 0.011 & 0.004 & 0.18 & 0.088 & 0.228 & 4.36 & 1.82 & 4.86 & 6.28 & 0.028 \\
\hline 1158 & High-Nickel (Ni 36) & 0.025 & 0.468 & 0.004 & 0.005 & 0.194 & 0.039 & 36.03 & 0.062 & 0.001 & 0.010 & & 0.008 \\
\hline 1233 & Valve Steel & 0.502 & 9.16 & 0.031 & 0.002 & 0.219 & 0.375 & 3.43 & 2108 & 0.096 & 0.237 & $(0.01)$ & N 0.415 \\
\hline
\end{tabular}




\section{High-Temperature Alloys}

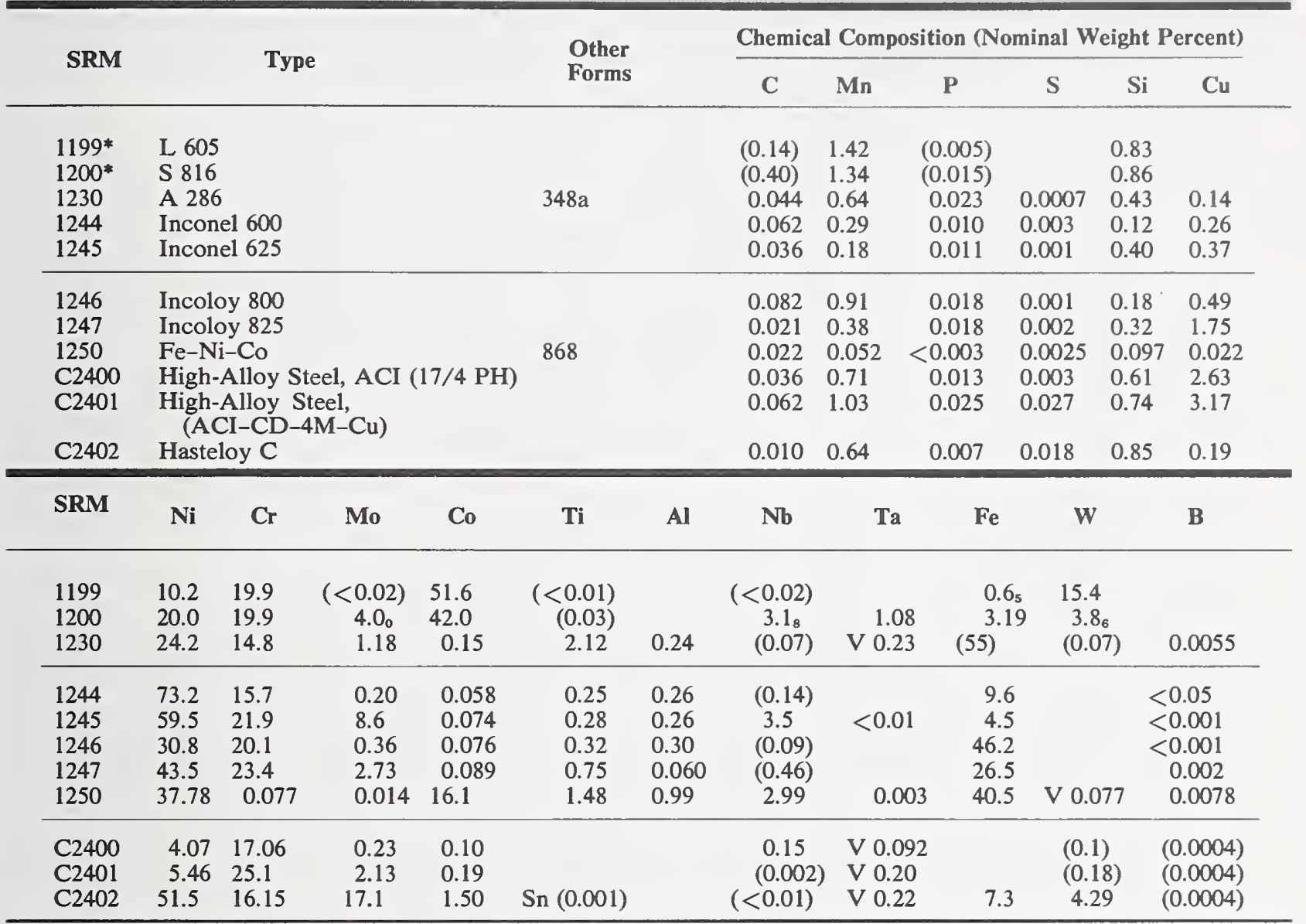

Values in parentheses are not certified, but are given for information only.

*SRM's 1199 and 1200 sold only in a set as S1199.

Bill Reed brings the qualitites of friendliness, resourcefulness and thoroughness to his new responsibility as the Acting Chief of the Office of Standard Reference Materials.

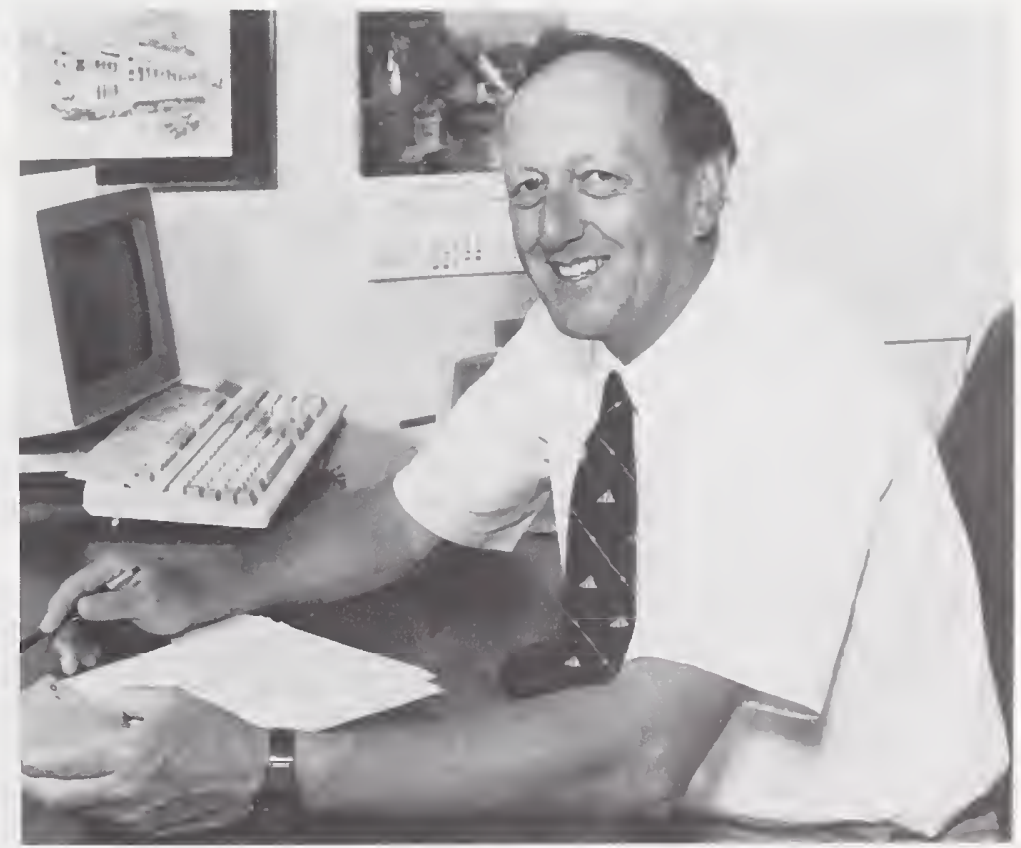




\section{Steelmaking Alloys}

These SRM's are for checking chemical methods of analysis for major constituents and for selected minor elements. They are furnished as fine powders (usually $<0.1 \mathrm{~mm}$ ).

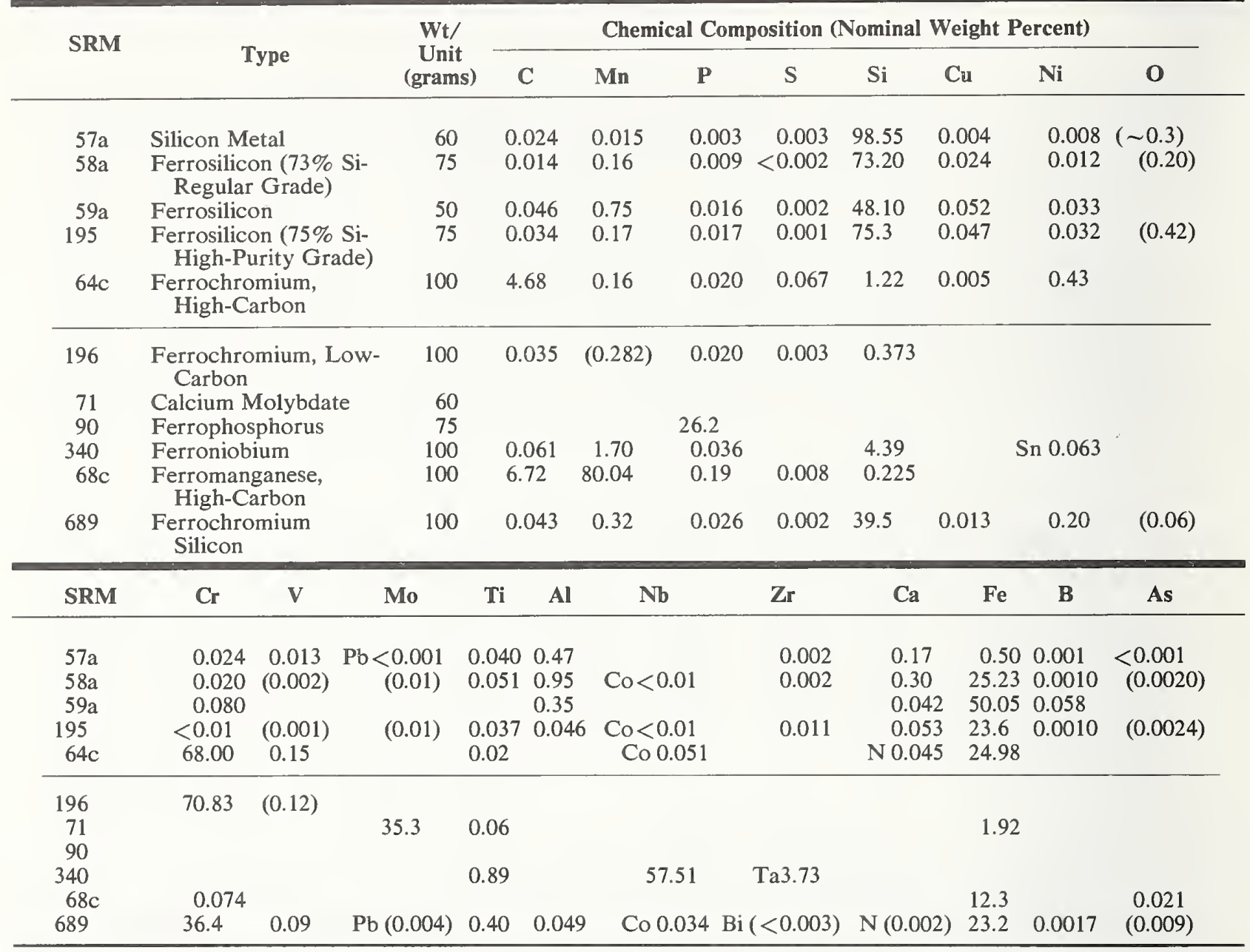

Values in parentheses are not certified, but are given for information only.

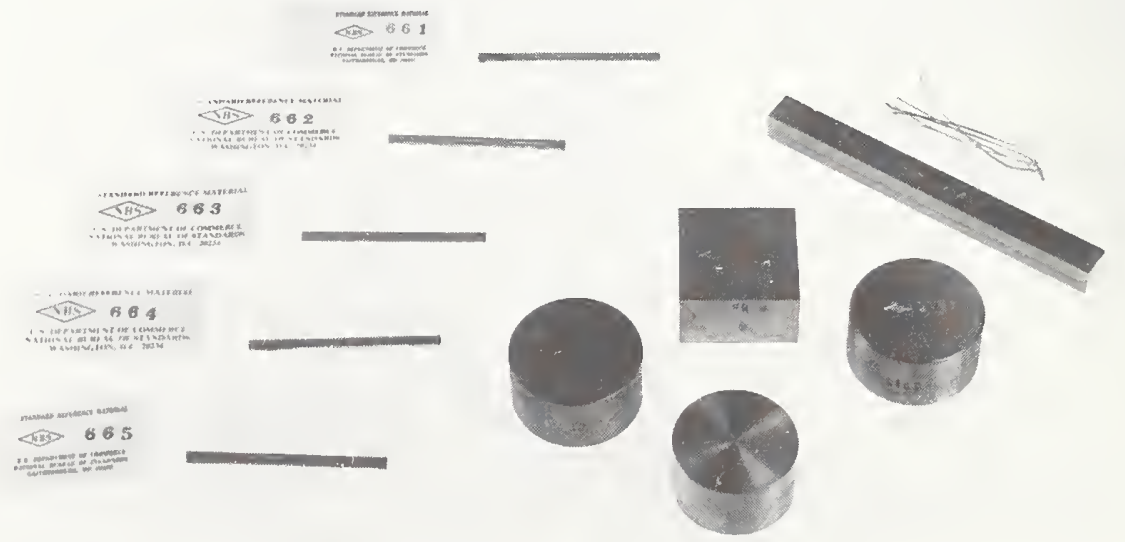

This group of steel SRM's illustrates the variety of forms of materials required to meet the SRM needs of the technical community. 


\section{Cast Irons (Chip Form)}

These SRM's are furnished in 150-g units (unless otherwise noted) for use in checking chemical methods of analysis.

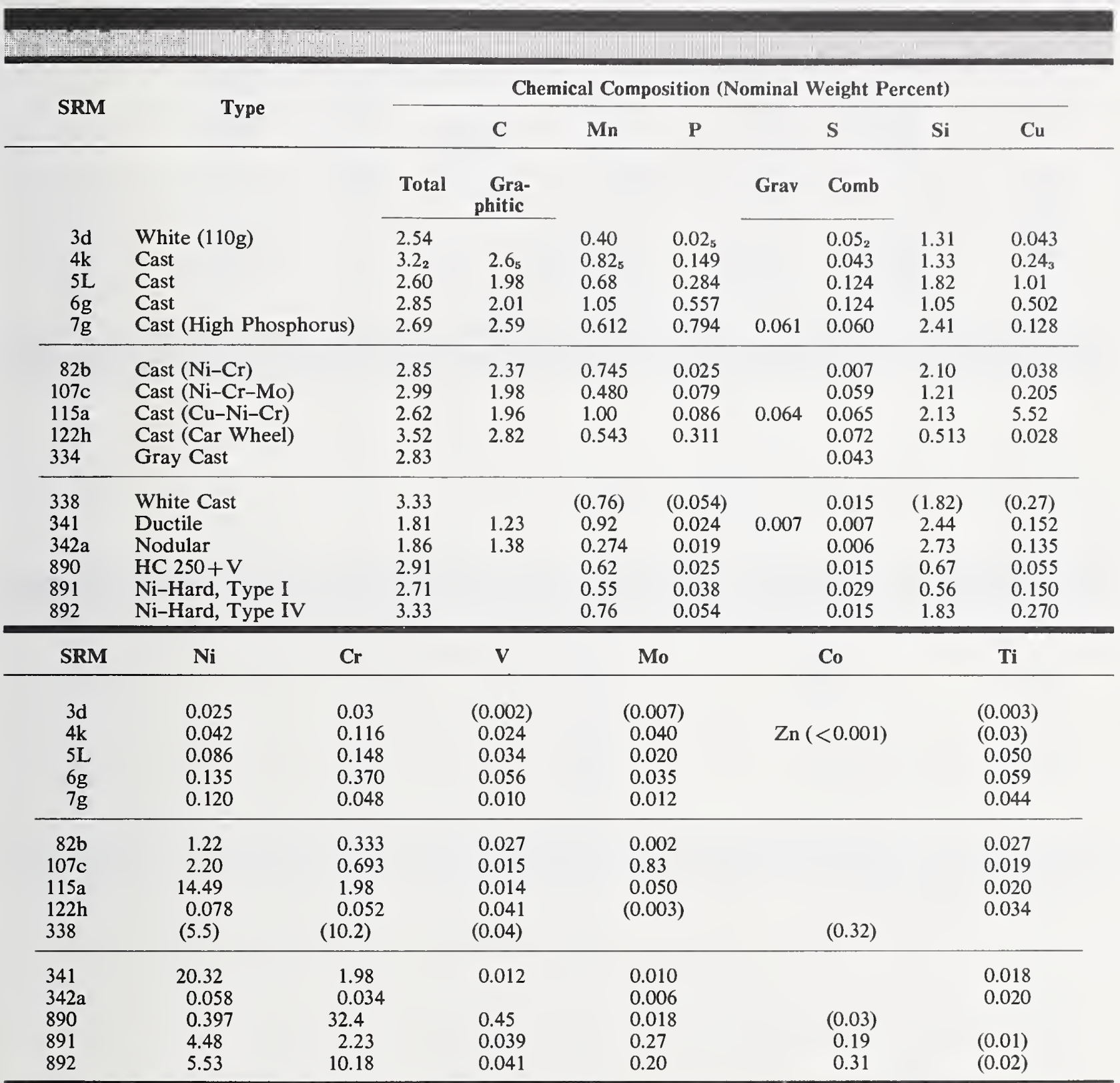

Values in parentheses are not certified, but are given for information only. 


\section{Cast Irons (Chip Form) (Continued)}

\begin{tabular}{|c|c|c|c|c|c|c|}
\hline SRM & As & Sn & Al (total) & $\mathbf{M g}$ & $\mathbf{N}$ & $\mathrm{Fe}$ \\
\hline $\begin{array}{r}4 \mathrm{k} \\
5 \mathrm{~L} \\
6 \mathrm{~g} \\
7 \mathrm{~g} \\
341\end{array}$ & $\begin{array}{l}(0.03) \\
0.042 \\
0.014\end{array}$ & $(0.004)$ & $(0.004)$ & $\begin{array}{r}\mathrm{Sb}(<0.001) \\
0.068\end{array}$ & $\begin{array}{l}(0.0016) \\
0.005 \\
0.005 \\
0.004\end{array}$ & $\mathrm{~Pb}(0.001)$ \\
\hline $\begin{array}{l}342 a \\
890 \\
891 \\
892\end{array}$ & $\begin{array}{l}(0.008) \\
(0.004) \\
(0.006)\end{array}$ & $\begin{array}{r}(<0.01) \\
(0.02)\end{array}$ & $\begin{array}{c}(<0.01) \\
(0.008) \\
(0.009)\end{array}$ & 0.070 & $\begin{array}{l}(0.089) \\
(0.012) \\
(0.019)\end{array}$ & $\begin{array}{l}(61.8) \\
(88.5) \\
(77.4)\end{array}$ \\
\hline
\end{tabular}

Values in parentheses are not certified, but are for information only.

\section{Cast Steels, White Cast Irons, Ductile Irons, and Blast Furnace Irons (Solid Form)}

These SRM's are for analysis of cast steels and cast irons by rapid instrumental methods.

\begin{tabular}{|c|c|c|c|c|c|c|c|c|c|}
\hline \multirow{2}{*}{ SRM } & \multirow{2}{*}{ Type } & \multicolumn{8}{|c|}{ Chemical Composition (Nominal Weight Percent) } \\
\hline & & $\mathbf{C}$ & Mn & $\mathbf{P}$ & $\mathbf{S}$ & Si & $\mathrm{Cu}$ & $\mathbf{N i}$ & $\mathrm{Cr}$ \\
\hline $\mathrm{C} 1137 \mathrm{a}$ & White Cast Iron & 2.86 & 0.52 & 0.087 & 0.017 & 1.15 & 0.192 & 2.17 & 0.643 \\
\hline $1138 \mathrm{a}$ & Cast Steel (No. 1) & $0.11_{8}$ & 0.35 & 0.035 & 0.056 & 0.25 & 0.09 & 0.10 & 0.13 \\
\hline $1139 \mathrm{a}$ & Cast Steel (No. 2) & $0.79_{0}$ & 0.92 & 0.012 & 0.013 & 0.80 & 0.47 & 0.98 & $2.1_{8}$ \\
\hline $1144 \mathrm{a}$ & Blast Furnace Iron (2) & 4.32 & 1.23 & $0.08_{4}$ & 0.083 & $0.18_{2}$ & 0.09 , & $0.06_{3}$ & 0.029 \\
\hline $\mathrm{C} 1145 \mathrm{a}$ & White Cast Iron & 2.92 & 0.187 & 0.215 & 0.191 & 0.271 & 0.46 & 0.62 & 0.63 \\
\hline $\mathrm{C} 1146 \mathrm{a}$ & White Cast Iron & 1.97 & 1.60 & 0.55 & 0.016 & 3.93 & 1.48 & 3.07 & 2.56 \\
\hline $\mathrm{C} 1150 \mathrm{a}$ & White Cast Iron & 3.32 & 0.77 & 0.078 & 0.065 & 1.35 & 0.112 & 0.097 & 0.155 \\
\hline $\mathrm{C} 1173$ & Cast Steel 3 & 0.453 & 0.174 & 0.031 & 0.092 & 1.38 & 0.204 & 4.04 & 2.63 \\
\hline 1173 & $\mathrm{Ni}-\mathrm{Cr}-\mathrm{Mo}-\mathrm{V}$ Steel & 0.423 & 0.19 & 0.033 & 0.092 & 1.28 & 0.204 & 4.06 & 2.70 \\
\hline C1290 & High Alloy $(\mathrm{HC}-250+\mathrm{V})$ & 3.04 & 0.66 & 0.030 & 0.013 & 0.971 & 0.065 & 0.917 & 30.5 \\
\hline C1291 & High Alloy (Ni-Hard, Type I) & 2.67 & 1.14 & 0.028 & 0.032 & 1.34 & 0.26 & 4.34 & 2.78 \\
\hline $\mathrm{C} 1292$ & High Alloy (Ni-Hard, Type IV) & 3.47 & 0.55 & 0.049 & 0.016 & 0.59 & 0.36 & 5.04 & 11.4 \\
\hline $\mathrm{C} 2423$ & Ductile Iron & 3.76 & 0.98 & 0.27 & $(0.0006)$ & 1.67 & 1.55 & 0.146 & 0.322 \\
\hline $\mathrm{C} 2423 \mathrm{a}$ & Ductile Iron & 3.66 & 0.91 & 0.246 & $(<0.001)$ & 1.59 & 1.61 & 0.147 & 0.322 \\
\hline $\mathrm{C} 2424$ & Ductile Iron & 2.68 & 0.268 & 0.041 & 0.024 & 3.37 & 0.125 & 0.061 & 0.13 \\
\hline $\mathrm{C} 2424 \mathrm{a}$ & Ductile Iron & 2.76 & 0.207 & 0.034 & 0.016 & 3.30 & 0.099 & 0.045 & 0.15 \\
\hline $\mathrm{C} 2425$ & Ductile Iron & 3.26 & 0.76 & 0.191 & 0.012 & 2.50 & 0.47 & 0.55 & 0.092 \\
\hline $\mathrm{C} 2425 \mathrm{a}$ & Ductile Iron & 3.30 & 0.72 & 0.188 & 0.010 & 2.38 & 0.47 & 0.57 & 0.085 \\
\hline
\end{tabular}




\section{Cast Steels, White Cast Irons, Ductile Irons, \\ and Blast Furnace Irons (Solid Form) (Continued)}

\begin{tabular}{|c|c|c|c|c|c|c|c|}
\hline SRM & v & Mo & $\mathbf{T i}$ & As & Al & Te & $\mathrm{Co}_{0}$ \\
\hline $\begin{array}{r}C 1137 a \\
1138 a \\
1139 a \\
1144 a \\
C 1145 a\end{array}$ & $\begin{array}{l}0.019 \\
0.02_{0} \\
0.26 \\
0.02_{5} \\
0.112\end{array}$ & $\begin{array}{c}0.86 \\
0.05 \\
0.51 \\
(0.007) \\
0.48\end{array}$ & $\begin{array}{l}(0.04) \\
(0.0012) \\
(0.004) \\
0.32 \\
0.012\end{array}$ & $\begin{array}{c}(<0.005) \\
(<0.005) \\
\quad(0.004) \\
(0.02)\end{array}$ & $\begin{array}{c}(0.007) \\
(0.067) \\
(0.13) \\
(<0.005) \\
(0.04)\end{array}$ & $\begin{array}{c}\mathrm{Mg} 0.032 \\
\mathrm{Fe}(98.7) \\
\mathrm{Fe}(93.0) \\
0.02_{2}\end{array}$ & Ce 0.016 \\
\hline $\begin{array}{c}\text { C1146a } \\
\text { C1150a } \\
\text { C1173 } \\
1173 \\
\text { C1290 }\end{array}$ & $\begin{array}{l}0.20 \\
0.040 \\
0.42 \\
0.42 \\
0.442\end{array}$ & $\begin{array}{l}1.52 \\
0.086 \\
1.46 \\
1.50 \\
(0.041)\end{array}$ & $\begin{array}{l}0.20 \\
0.040 \\
0.037 \\
(0.015)\end{array}$ & $\begin{array}{l}(0.16) \\
(0.017) \\
(0.02)\end{array}$ & $\begin{array}{l}(0.028) \\
(0.005) \\
(0.005)\end{array}$ & $\begin{array}{c}\mathrm{Pb} 0.0018 \\
\mathrm{~Pb} 0.001 \\
\mathrm{~Pb}(0.0006) \\
\mathrm{Nb}(0.045)\end{array}$ & $\begin{array}{l}0.13 \\
0.014 \\
0.064 \\
0.076\end{array}$ \\
\hline $\begin{array}{l}\text { C1291 } \\
\text { C1292 } \\
\text { C2423 } \\
\text { C2423a } \\
\text { C2424 }\end{array}$ & $\begin{array}{l}0.031 \\
0.041 \\
0.048 \\
0.043 \\
0.083\end{array}$ & $\begin{array}{l}0.32 \\
0.25 \\
0.155 \\
0.159 \\
0.019\end{array}$ & $\begin{array}{l}0.10 \\
0.10 \\
0.050\end{array}$ & & $\begin{array}{r}(0.09) \\
(0.08) \\
(<0.01)\end{array}$ & & $\begin{array}{l}(0.02) \\
(0.02) \\
(0.05)\end{array}$ \\
\hline $\begin{array}{l}\text { C2424a } \\
\text { C2425 } \\
\text { C2425a }\end{array}$ & $\begin{array}{l}0.081 \\
0.013 \\
0.013\end{array}$ & $\begin{array}{l}0.019 \\
0.30 \\
0.29\end{array}$ & $\begin{array}{l}0.045 \\
0.19 \\
0.20\end{array}$ & & $\begin{array}{r}(<0.01) \\
(0.02) \\
(0.02)\end{array}$ & & $\begin{array}{l}(0.05) \\
(0.02) \\
(0.03)\end{array}$ \\
\hline SRM & & $\mathbf{M g}$ & & $\mathrm{Ce}$ & & $\mathrm{La}$ & B \\
\hline $\begin{array}{l}\text { C2423 } \\
\text { C2423a } \\
\text { C2424 }\end{array}$ & & $\begin{array}{l}0.058 \\
0.076 \\
0.006\end{array}$ & & $\begin{array}{l}0.036 \\
0.031 \\
0.0046\end{array}$ & & $\begin{array}{l}011 \\
0042 \\
0011\end{array}$ & $\begin{array}{l}(0.01) \\
(0.01) \\
(0.002)\end{array}$ \\
\hline $\begin{array}{l}\text { C2424a } \\
\text { C2425 } \\
\text { C2425a }\end{array}$ & & $\begin{array}{l}0.014 \\
0.040 \\
0.047\end{array}$ & & $\begin{array}{l}0.0053 \\
0.0062 \\
0.023\end{array}$ & & $\begin{array}{l}0010 \\
0015 \\
0037\end{array}$ & $\begin{array}{l}(0.001) \\
(0.10) \\
(0.1)\end{array}$ \\
\hline
\end{tabular}

Values in parentheses are not certified, but are given for information only. 


\section{Aluminum-Base Alloys}

\begin{tabular}{|c|c|c|c|c|c|c|c|c|c|c|}
\hline \multirow{2}{*}{ SRM } & \multirow{2}{*}{\multicolumn{3}{|c|}{ Type }} & \multirow{2}{*}{$\begin{array}{c}\text { Wt/ } \\
\text { Unit } \\
\text { (grams) }\end{array}$} & \multicolumn{6}{|c|}{ Chemical Composition (Nominal Weight Percent) } \\
\hline & & & & & Mn & Si & $\mathrm{Cu}$ & $\mathbf{N i}$ & $\mathbf{C r}$ & $\mathbf{v}$ \\
\hline $87 a$ & \multirow{2}{*}{\multicolumn{3}{|c|}{ Al-Si (Chip) }} & 75 & 0.26 & 6.24 & 0.30 & 0.57 & 0.11 & $<0.01$ \\
\hline 853 & & & & \multirow{2}{*}{$\begin{array}{r}30 \\
\text { Disk }\end{array}$} & 1.26 & 0.18 & 0.15 & 0.004 & $<0.001$ & 0.017 \\
\hline 1240 & \multicolumn{3}{|c|}{ Alloy 3004} & & 1.26 & 0.18 & 0.15 & 0.004 & $<0.001$ & 0.017 \\
\hline $1240 \mathrm{a}$ & \multicolumn{3}{|l|}{ Alloy 3004} & Disk & 1.27 & 0.18 & 0.15 & 0.004 & $<0.001$ & 0.017 \\
\hline $1240 \mathrm{~b}$ & \multicolumn{3}{|l|}{ Alloy 3004} & Disk & 1.27 & 0.18 & 0.15 & 0.004 & $<0.001$ & 0.017 \\
\hline 854 & \multicolumn{3}{|c|}{ Alloy 5182 (Chip) } & 30 & 0.38 & 0.16 & 0.050 & 0.020 & 0.032 & 0.016 \\
\hline $1241 \mathrm{a}$ & \multicolumn{3}{|c|}{ Alloy 5182} & Disk & 0.38 & 0.16 & 0.050 & 0.020 & 0.032 & 0.016 \\
\hline $1241 \mathrm{~b}$ & \multirow{2}{*}{\multicolumn{3}{|c|}{$\begin{array}{l}\text { Alloy } 5182 \\
\text { Casting Alloy } 356 \text { (fine millings) }\end{array}$}} & \multirow{2}{*}{$\begin{array}{r}\text { Disk } \\
30\end{array}$} & 0.38 & 0.16 & 0.050 & 0.020 & 0.032 & \multirow[t]{2}{*}{0.016} \\
\hline 855 & & & & & 0.057 & 7.17 & 0.13 & 0.015 & 0.013 & \\
\hline $1255 \mathrm{a}$ & \multirow{2}{*}{\multicolumn{3}{|c|}{$\begin{array}{l}\text { Casting Alloy } 356 \\
\text { Casting Alloy } 380 \text { (fine millings) }\end{array}$}} & \multirow{2}{*}{$\begin{array}{r}\text { Disk } \\
30\end{array}$} & 0.053 & 7.22 & 0.12 & 0.017 & 0.012 & 0.024 \\
\hline 856 & & & & & 0.35 & 9.21 & 3.51 & 0.37 & 0.055 & \\
\hline $1256 a$ & \multicolumn{3}{|c|}{ Casting Alloy 380} & Disk & 0.38 & 9.18 & 3.51 & 0.41 & 0.055 & 0.018 \\
\hline $\mathrm{C} 1257$ & \multicolumn{3}{|c|}{ High Purity } & Disk & $<1.0$ & $<10$ & $<1.0$ & $<1.0$ & $<1.0$ & $<0.1$ \\
\hline 858 & $\begin{array}{l}\text { Alloy } 6011 \text { ( } \\
\text { ings) }\end{array}$ & (modified) & (fine mill- & 35 & 0.48 & 0.79 & 0.84 & 0.0006 & 0.0011 & 0.0030 \\
\hline 1258 & $\begin{array}{l}\text { Alloy } 6011 \\
\text { thick) }\end{array}$ & $(35 \mathrm{~mm}$ & $\mathrm{D} \times 19 \mathrm{~mm}$ & Disk & 0.48 & 0.78 & 0.84 & 0.0006 & 0.0011 & \\
\hline 859 & Alloy 7075 (fi & fine millin & & 35 & 0.078 & 0.17 & 1.59 & 0.063 & 0.176 & 0.0082 \\
\hline 1259 & $\begin{array}{l}\text { Alloy } 7075 \\
\text { thick) }\end{array}$ & $(35 \mathrm{~mm}$ & $\mathrm{D} \times 19 \mathrm{~mm}$ & Disk & 0.079 & 0.18 & 1.60 & 0.063 & 0.173 & \\
\hline SRM & $\mathbf{T i}$ & Sn & $\mathrm{Ga}$ & $\mathbf{F e}$ & $\mathbf{P b}$ & & $\mathbf{M g}$ & $\mathrm{Zn}$ & $\mathrm{Zr}$ & $\mathbf{B e}$ \\
\hline $87 a$ & 0.18 & 0.05 & 0.02 & 0.61 & 0.10 & & 0.37 & 0.16 & & \\
\hline 853 & 0.018 & & 0.018 & 0.50 & & & 1.11 & 0.052 & 0.002 & - \\
\hline 1240 & 0.022 & & 0.018 & 0.50 & & & 1.11 & 0.052 & 0.002 & \\
\hline $1240 \mathrm{a}$ & 0.022 & & 0.018 & 0.50 & & & 1.12 & 0.051 & 0.002 & \\
\hline $1240 \mathrm{~b}$ & 0.021 & & 0.018 & 0.50 & & & 1.11 & 0.051 & 0.002 & \\
\hline 854 & 0.030 & & 0.018 & 0.20 & & & 4.54 & 0.051 & 0.002 & \\
\hline $1241 \mathrm{a}$ & 0.032 & & 0.018 & 0.20 & & & 4.54 & 0.052 & 0.002 & \\
\hline $1241 \mathrm{~b}$ & 0.034 & & 0.018 & 0.20 & & & 4.54 & 0.051 & 0.002 & \\
\hline 855 & 0.15 & 0.010 & & 0.16 & 0.015 & & 0.37 & 0.083 & 0.002 & \\
\hline $1255 \mathrm{a}$ & 0.156 & 0.013 & & 0.14 & 0.017 & & 0.36 & 0.083 & Sr 0.02 & \\
\hline 856 & 0.068 & 0.10 & & 0.92 & 0.10 & & 0.061 & 0.96 & & \\
\hline $1256 a$ & 0.084 & 0.10 & & 0.90 & 0.10 & & 0.062 & 1.02 & Sr 0.020 & \\
\hline $\mathrm{C} 1257$ & $(<0.1)$ & $(<0.1)$ & $(<0.1)$ & 1.0 & $(<0.1)$ & & $<1.0$ & $(<0.1)$ & $(<0.1)$ & $(<0.1)$ \\
\hline 858 & 0.042 & & & 0.078 & & & 1.01 & 1.04 & & $<0.0001$ \\
\hline 1258 & $(0.04)$ & & $(0.010)$ & 0.079 & & & 0.98 & 1.03 & & $<0.0001$ \\
\hline 859 & 0.041 & & & 0.202 & & & 2.45 & 5.46 & & 0.0026 \\
\hline 1259 & $(0.04)$ & & $(0.022)$ & 0.205 & & & 2.48 & 5.44 & & 0.0025 \\
\hline
\end{tabular}

Values in parentheses are not certified, but are given for information only. 


\section{Copper-Base Alloys (Chip Form)}

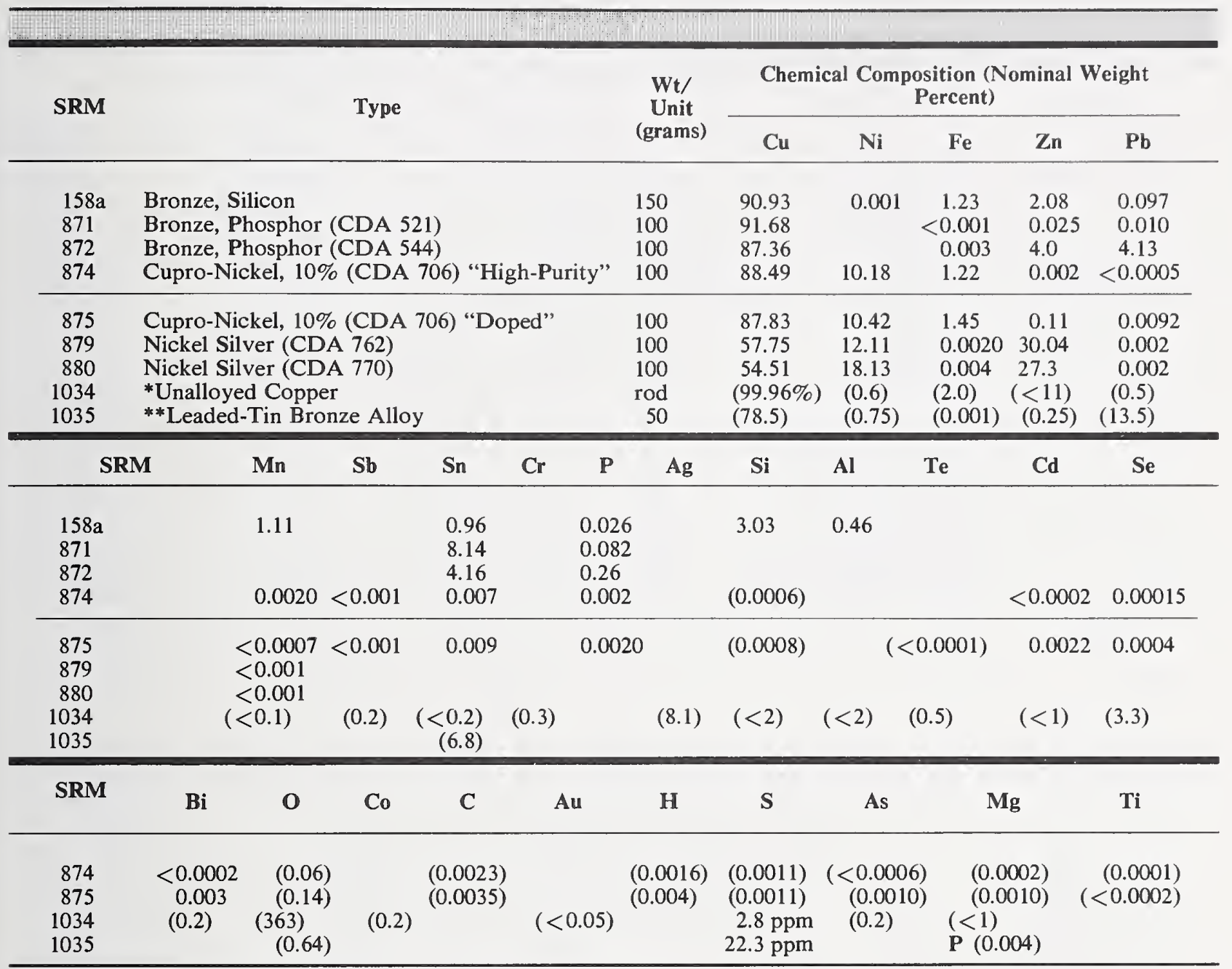

Values in parentheses are not certified, but are given for information only.

*Values for SRM 1034 are ppm by weight.

**Sulfur value for SRM 1035 is ppm by weight. 


\section{Copper-Base Alloys (Solid Form)}

The SRM's with "C" prefix are chill-cast blocks, $31 \mathrm{~mm}$ square, $19 \mathrm{~mm}$ thick; the others are wrought disks, $31 \mathrm{~mm}$ in diameter and $19 \mathrm{~mm}$ thick. Both forms have nearly identical chemical compositions.

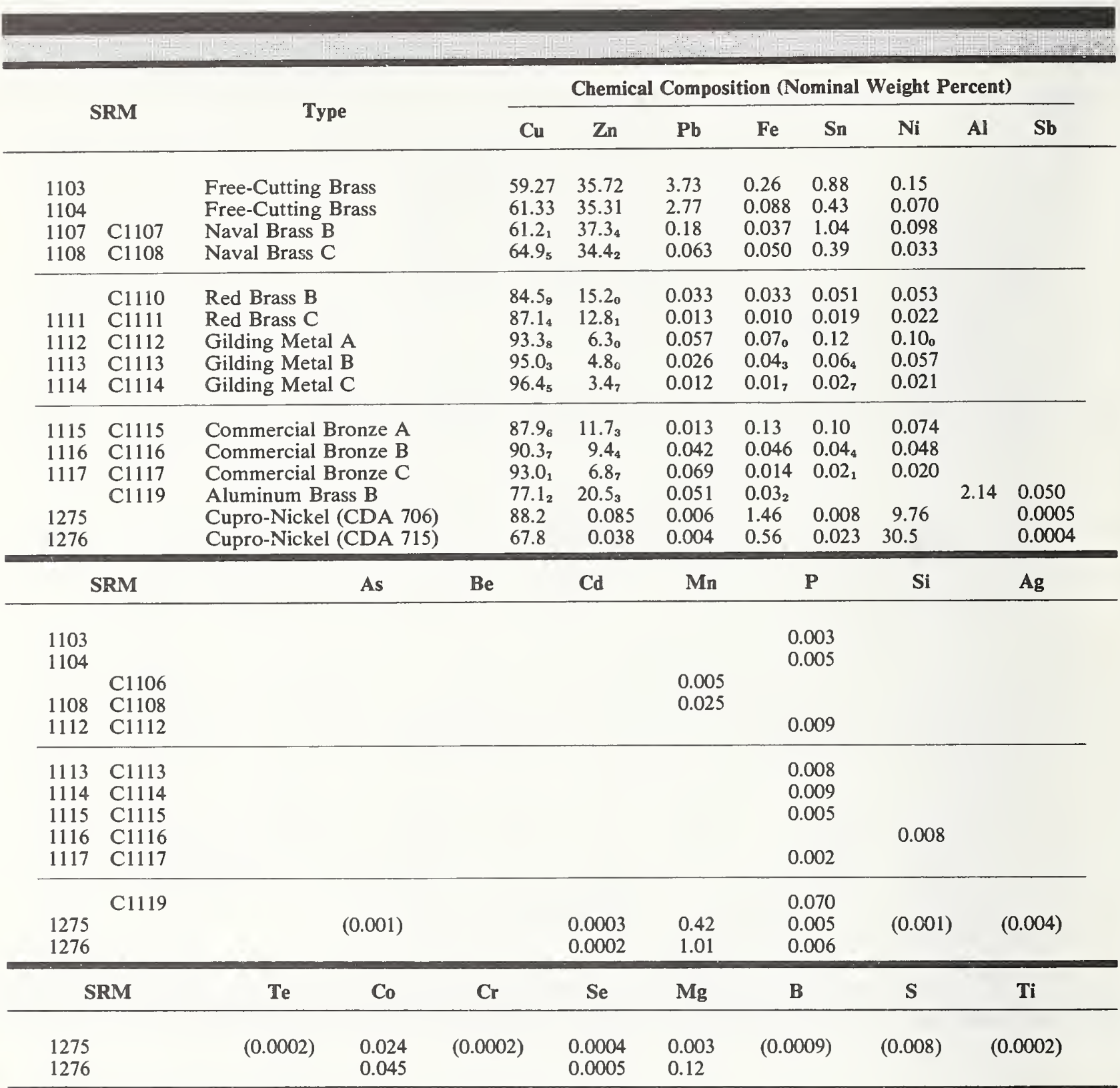

Values in parentheses are not certified, but are given for information only. 


\section{Copper "Benchmark"}

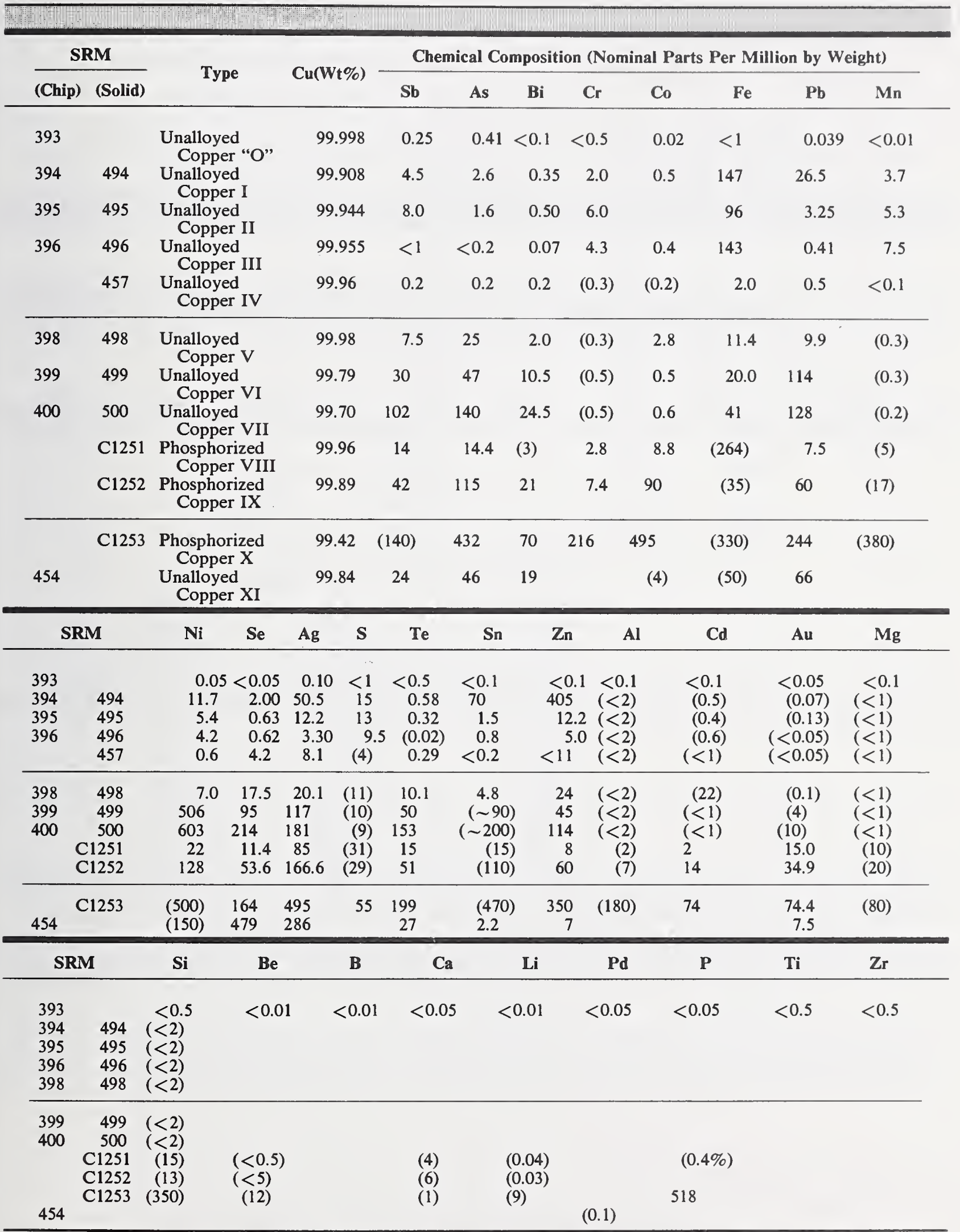

Values in parentheses are not certified, but are given for information only. 


\section{Lead-Base Alloys}

\begin{tabular}{|c|c|c|c|c|c|c|c|c|c|c|}
\hline \multicolumn{2}{|c|}{ SRM } & \multirow{2}{*}{ Type } & \multicolumn{8}{|c|}{ Chemical Composition (Nominal Weight Percent) } \\
\hline Chip & Disk & & $\mathrm{Cu}$ & $\mathbf{N i}$ & As & Sn & $\mathrm{Sb}$ & $\mathbf{B i}$ & $\mathrm{Ag}$ & $\mathbf{F e}$ \\
\hline 1129 & & Solder $63 \mathrm{Sn}-37 \mathrm{~Pb}$ & 0.16 & 0.010 & 0.055 & 62.7 & 0.13 & 0.13 & 0.075 & \\
\hline $127 \mathrm{~b}$ & 1131 & Solder $60 \mathrm{~Pb}-40 \mathrm{Sn}$ & 0.011 & 0.012 & 0.01 & 39.3 & 0.43 & 0.06 & 0.01 & \\
\hline $53 e$ & 1132 & Bearing Metal( $\mathrm{Pb}-\mathrm{Sb}-\mathrm{Sn})$ & 0.054 & 0.003 & 0.057 & 5.84 & 10.26 & 0.052 & & $<0.001$ \\
\hline
\end{tabular}

\section{Lead-Base Material}

\begin{tabular}{|c|c|c|c|}
\hline SRM & C2416 & C2417 & C2418 \\
\hline Type & Bullet Lead & Lead-Base Alloy & High-Purity Lead \\
\hline Size & $50 \mathrm{~mm}$ & $50 \mathrm{~mm}$ & $50 \mathrm{~mm}$ \\
\hline
\end{tabular}

Chemical Composition (Nominal Weight Percent)

\begin{tabular}{lccr}
\hline $\mathrm{Sb}$ & 0.79 & 0.010 & $(<0.0001)$ \\
$\mathrm{As}$ & 0.056 & 0.011 & $(<0.0001)$ \\
$\mathrm{Bi}$ & 0.10 & 0.010 & $(<0.0005)$ \\
$\mathrm{Cu}$ & 0.065 & 0.010 & $(<0.0001)$ \\
$\mathrm{S}$ & 0.0015 & $(<0.0005)$ & - \\
$\mathrm{Ag}$ & 0.0044 & 0.010 & 0.0001 \\
$\mathrm{Sn}$ & 0.09 & $(<0.010)$ & $(<0.0005)$ \\
$\mathrm{Al}$ & $(<0.0001)$ & $(<0.0001)$ & $(<0.0001)$ \\
$\mathrm{Cd}$ & $(0.0002)$ & $(<0.0002)$ & $(<0.0003$ \\
$\mathrm{Ca}$ & $(<0.001)$ & $(<0.0002)$ & $(<0.0005)$ \\
$\mathrm{Co}$ & $(<0.0002)$ & $(<0.0003)$ & $(<0.0005)$ \\
$\mathrm{Fe}$ & $(<0.0005)$ & $(<0.0003)$ & $(<0.0005)$ \\
$\mathrm{Mn}$ & $(<0.0005)$ & $(<0.0005)$ & $(<0.0005)$ \\
$\mathrm{Ni}$ & $(<0.0005)$ & $(<0.0005)$ & $(<0.0005)$ \\
$\mathrm{Te}$ & $(<0.0005)$ & $(<0.0005)$ & $(<0.0005)$ \\
$\mathrm{Zn}$ & $(<0.0005)$ & &
\end{tabular}

These lead-base materials are issued in the form of disks $50 \mathrm{~mm}$ in diameter and $16 \mathrm{~mm}$ thick. They are intended for use with optical emission spectrometric methods of analysis. 


\section{Nickel-Base Alloys}

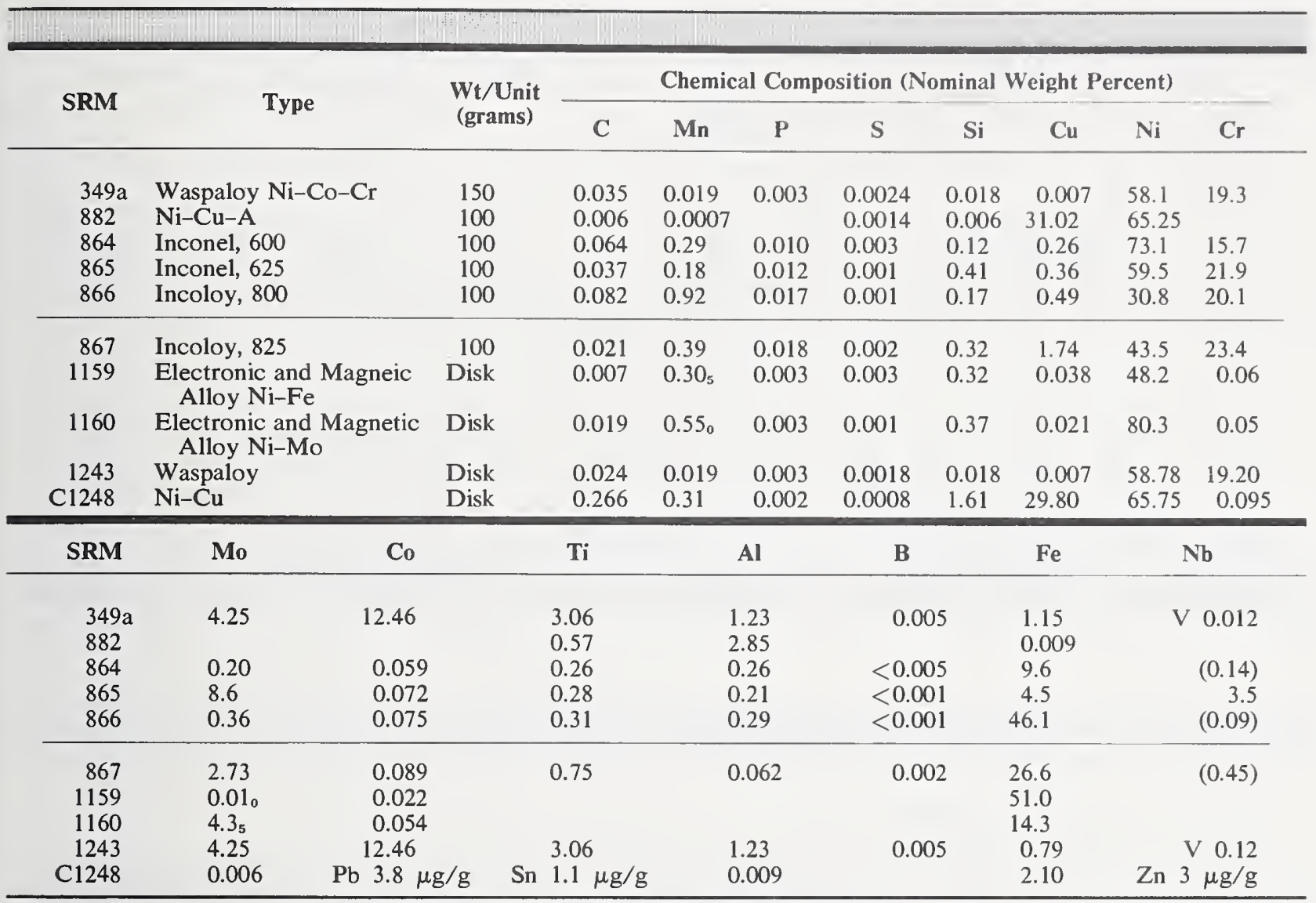

Values in parentheses are not certified, but are given for information only.

\section{Carbon Modified Silicon}

SRM 1216-This SRM is intended for the calibration of instruments used to measure total elemental carbon. The SRM consists of three, one gram bottles of chemically modified microparticulate silica certified at the carbon levels of $0.70,9.06$, and $17.04 \%$ carbon, respectively.

\begin{tabular}{|c|c|c|c|}
\hline SRM & Type & Bottle & Percent Carbon \\
\hline 1216 & Carbon Modified Silicon & $\begin{array}{c}\text { I } \\
\text { II } \\
\text { III }\end{array}$ & $\begin{array}{c}0.070 \\
9.06 \\
17.04\end{array}$ \\
\hline
\end{tabular}




\section{Trace Elements in Nickel-Base Superalloys (Chip Form)}

\begin{tabular}{|c|c|c|c|c|c|c|c|c|c|c|c|c|}
\hline \multirow{2}{*}{ SRM } & \multirow{2}{*}{\multicolumn{3}{|c|}{ Type }} & & \multirow{2}{*}{\multicolumn{2}{|c|}{$\begin{array}{l}\text { Wt/Unit } \\
\text { (grams) }\end{array}$}} & \multicolumn{6}{|c|}{$\begin{array}{c}\text { Nominal Trace Composition (Parts Per Million by } \\
\text { Weight) }\end{array}$} \\
\hline & & & & & & & $\mathbf{P b}$ & $\mathbf{B i}$ & & & $\mathrm{Te}$ & Tl \\
\hline 897 & \multicolumn{3}{|c|}{ "Tracealloy" A } & & \multicolumn{2}{|c|}{35} & 11.7 & $(0.5)$ & \multicolumn{2}{|c|}{9.1} & 1.05 & 0.51 \\
\hline 898 & \multicolumn{3}{|c|}{ "Tracealloy" B } & & \multicolumn{2}{|c|}{35} & 2.5 & (1.0) & \multicolumn{2}{|c|}{2.00} & 0.54 & 2.75 \\
\hline 899 & & raceallo & & & \multicolumn{2}{|c|}{35} & 3.9 & $(0.3)$ & \multicolumn{2}{|c|}{9.5} & 5.9 & 0.252 \\
\hline \multirow{2}{*}{ SRM } & \multicolumn{12}{|c|}{ Approximate Base Composition (Weight Percent) } \\
\hline & C & $\mathrm{Cr}$ & Co & $\mathbf{N i}$ & $\mathbf{w}$ & $\mathbf{N b}$ & Al & Ti & $\mathbf{B}$ & $\mathbf{Z r}$ & Ta & Hf \\
\hline 897 & $(0.12)$ & $(12.0)$ & $(8.5)$ & (Bal) & $(1.75)$ & $(0.9)$ & $(2.0)$ & $(2.0)$ & $(0.010)$ & $(0.10)$ & (1.75) & (1.2) \\
\hline 898 & $(0.12)$ & (12.0) & (8.5) & $(\mathrm{Bal})$ & $(1.75)$ & $(0.9)$ & $(2.0)$ & (2.0) & $(0.010)$ & $(0.10)$ & $(1.75)$ & (1.2) \\
\hline 899 & $(0.12)$ & (12.0) & $(8.5)$ & $(\mathrm{Bal})$ & (1.75) & (0.9) & $(2.0)$ & (2.0) & $(0.010)$ & $(0.10)$ & $(1.75)$ & (1.2) \\
\hline
\end{tabular}

Values in parentheses are not certified, but are given for information only.

\section{Nickel Oxides (Powder Form)}

\begin{tabular}{|c|c|c|c|c|c|c|c|c|c|c|c|c|c|c|}
\hline \multirow{2}{*}{ SRM } & \multirow{2}{*}{\multicolumn{2}{|c|}{ Type }} & & \multirow{2}{*}{\multicolumn{2}{|c|}{$\begin{array}{l}\text { Wt/Unit } \\
\text { (grams) }\end{array}$}} & \multicolumn{9}{|c|}{ Chemical Composition (Nominal Weight Percent) } \\
\hline & & & & & & Mn & Si & $\mathrm{Cu}$ & $\mathbf{C r}$ & Co & $\mathrm{Ti}$ & Al & $\mathrm{Fe}$ & $\mathbf{M g}$ \\
\hline 671 & Oxi & le 1 & & & 5 & 0.13 & 0.047 & 0.20 & 0.025 & 0.31 & 0.024 & 0.009 & 0.39 & 0.030 \\
\hline 672 & Oxi & le 2 & & & 5 & 0.095 & 0.11 & 0.018 & 0.003 & 0.55 & 0.009 & 0.004 & 0.079 & 0.020 \\
\hline 673 & Oxi & le 3 & & & 5 & 0.0037 & 0.006 & 0.002 & 0.0003 & 0.016 & 0.003 & 0.001 & 0.029 & 0.003 \\
\hline \multirow{2}{*}{ SRM } & \multicolumn{14}{|c|}{ Nominal Trace Composition (Parts Per Million by Weight) } \\
\hline & $\mathbf{P b}$ & Se & $\mathbf{B i}$ & As & Sn & & b & $\mathrm{Cd}$ & Ga & Ag & $\mathrm{Te}$ & & [1 & $\mathrm{Zn}$ \\
\hline 671 & 16 & 2.0 & 0.07 & (59) & $(2.7)$ & & $0.4)$ & $(0.7)$ & $(0.8)$ & $(0.5)$ & $(<0.2)$ & & $0.1)$ & (160) \\
\hline 672 & 38 & 0.40 & 0.3 & (74) & (4) & & $0.5)$ & (1.7) & $(0.4)$ & $(0.3)$ & $(<0.2)$ & & $0.1)$ & (140) \\
\hline 673 & 3.5 & 0.2 & 0.06 & $(0.4)$ & $(<0.5)$ & & $0.5)$ & $(0.05)$ & $(<0.1)$ & $(<0.1)$ & $(0.4)$ & & $0.1)$ & (1.7) \\
\hline
\end{tabular}

Values in parentheses are not certified, but are given for information only. 


\section{Titanium-Base Alloys}

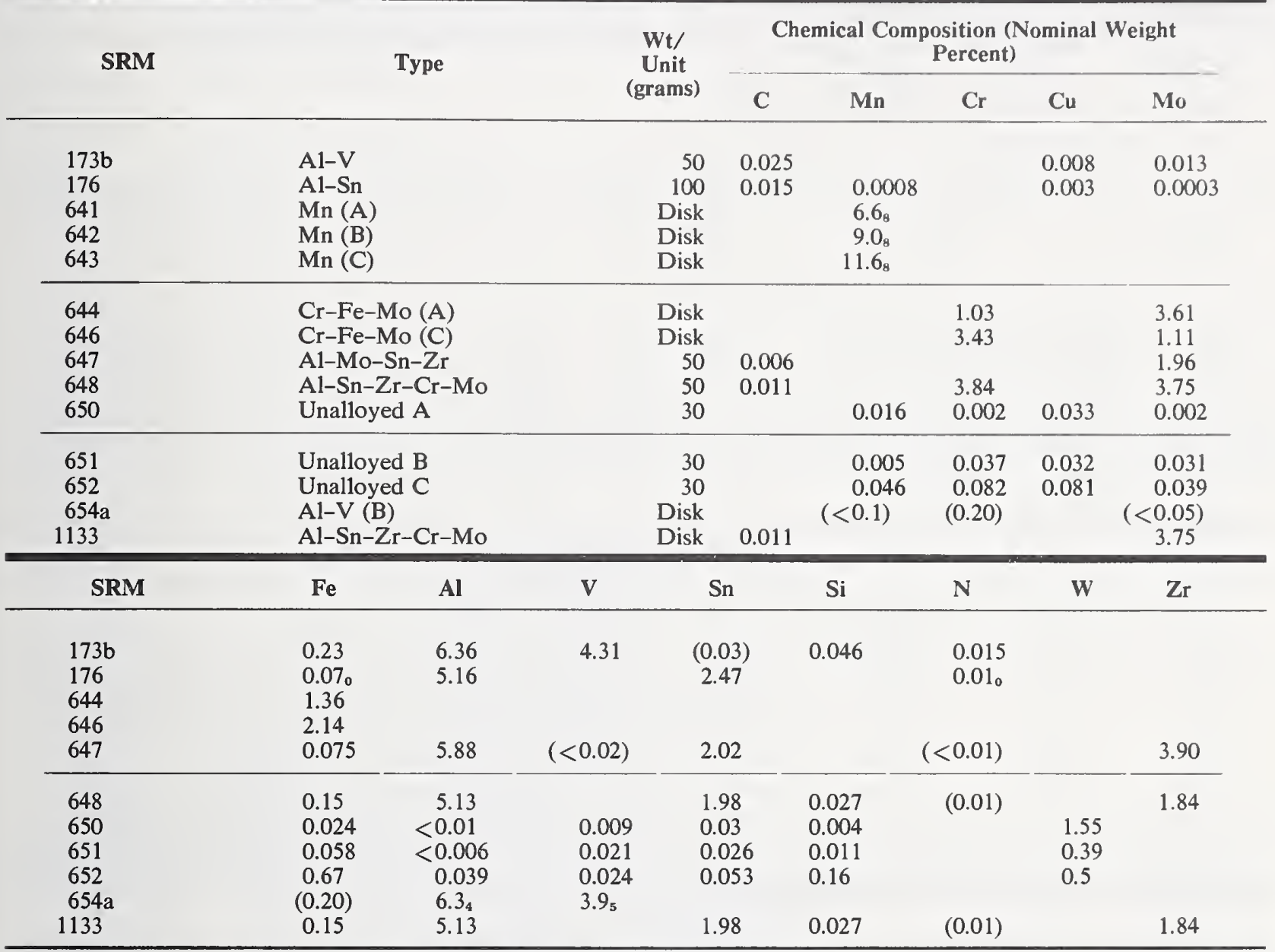

Values in parentheses are not certified, but are given for information only. 


\section{Zinc-Base Alloys}

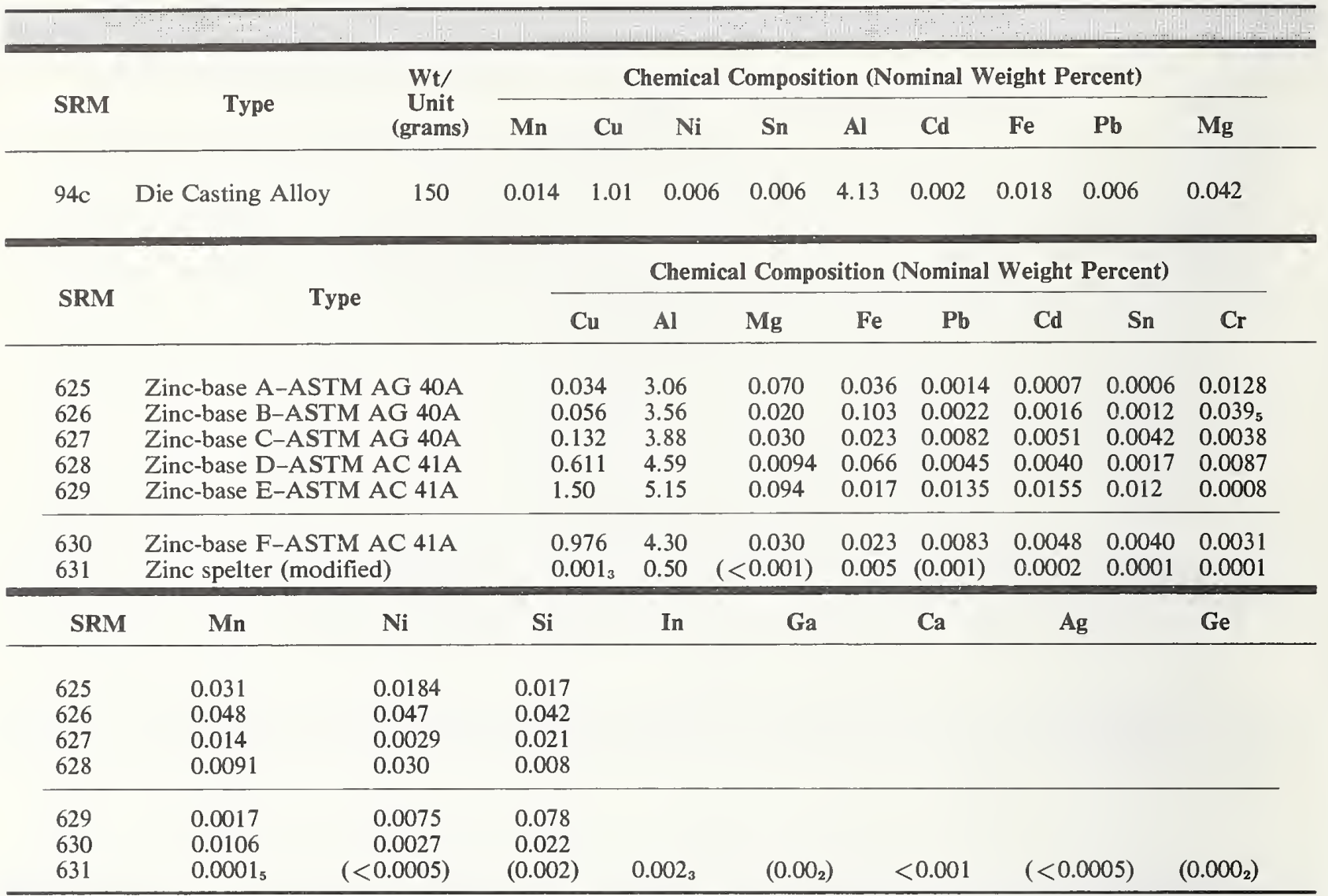

Values in parentheses are not certified, but are given for information only.

\section{Zirconium-Base Alloys}

\begin{tabular}{|c|c|c|c|c|c|c|c|c|c|c|c|c|c|c|}
\hline \multirow{2}{*}{ SRM } & \multirow{2}{*}{ Type } & \multirow{2}{*}{$\begin{array}{c}\text { Wt/Unit } \\
\text { (grams) }\end{array}$} & \multicolumn{12}{|c|}{ Chemical Composition (Nominal Weight Percent) } \\
\hline & & & C & Mn & Hf & $\mathrm{Cu}$ & $\mathrm{Ni}$ & $\mathrm{Cr}$ & $\mathbf{T i}$ & Sn & & $\mathrm{Fe}$ & $\mathbf{N}$ & Al \\
\hline $360 \mathrm{~b}$ & Zircaloy -4 & 100 & 0.011 & 0.0010 & 0.008 & 0.002 & 0.0025 & 0.10 & 0.002 & 1.55 & & $0.21 \mathrm{C}$ & 0.0045 & 0.004 \\
\hline \multirow{2}{*}{\multicolumn{2}{|c|}{$\begin{array}{c}\text { SRM } \\
31 \mathrm{~mm} \mathrm{D \times} \\
9.5 \mathrm{~mm} \text { thick }\end{array}$}} & \multirow{2}{*}{ Type } & \multicolumn{12}{|c|}{ Chemical Composition (Nominal Parts Per Million) } \\
\hline & & & Hf & $\mathbf{C}$ & & $\mathrm{Cu}$ & $\mathbf{F e}$ & Mn & Mo & $\mathbf{N i}$ & $\mathbf{N}$ & $\mathbf{S i}$ & $\mathbf{T i}$ & w \\
\hline \multirow{2}{*}{\multicolumn{2}{|c|}{$\begin{array}{l}1234 \\
123.7\end{array}$}} & conium A & $46 \quad(8$ & (80) & 55) & $<10)$ & $(240)$ & (10) & (2) & $(20)$ & (14) & $(40)$ & $(20)$ & $(25)$ \\
\hline & & & $31 \quad(10$ & 00) (151 & & $<10)$ & (1650) & (10) & $(<10)$ & (40) & (19) & (35) & (30) & (25) \\
\hline
\end{tabular}

Values in parentheses are not certified, but are given for information only. 


\section{Gases in Metals}

These SRM's are for determining hydrogen, oxygen, and nitrogen by vacuum fusion, inert gas fusion, and neutron activation methods. SRM's 1095 to 1099 are sold only in a set as SRM 1089.

\begin{tabular}{|c|c|c|c|c|c|}
\hline SRM & Type & Form & $\begin{array}{c}\text { Oxygen } \\
\text { (ppm) }\end{array}$ & $\begin{array}{c}\text { Hydrogen } \\
\text { (ppm) }\end{array}$ & $\begin{array}{c}\text { Nitrogen } \\
\text { (ppm) }\end{array}$ \\
\hline $352 \mathrm{c}$ & Unalloyed titanium for hydrogen & Platelets & & (IN PREP) & \\
\hline 1090 & Ingot iron & Rod & $(491)$ & & $(60)$ \\
\hline 1094 & Maraging steel & Rod & 4.5 & & (71) \\
\hline 1089 & Set of 5: 1095, 1096, 1097, 1098, and 1099 & Rods & & & \\
\hline 1754 & Low-Alloy Steel, AISI 4320 & Rod & 24 & & 81 \\
\hline
\end{tabular}

Values in parentheses are not certified, but are given for information only.

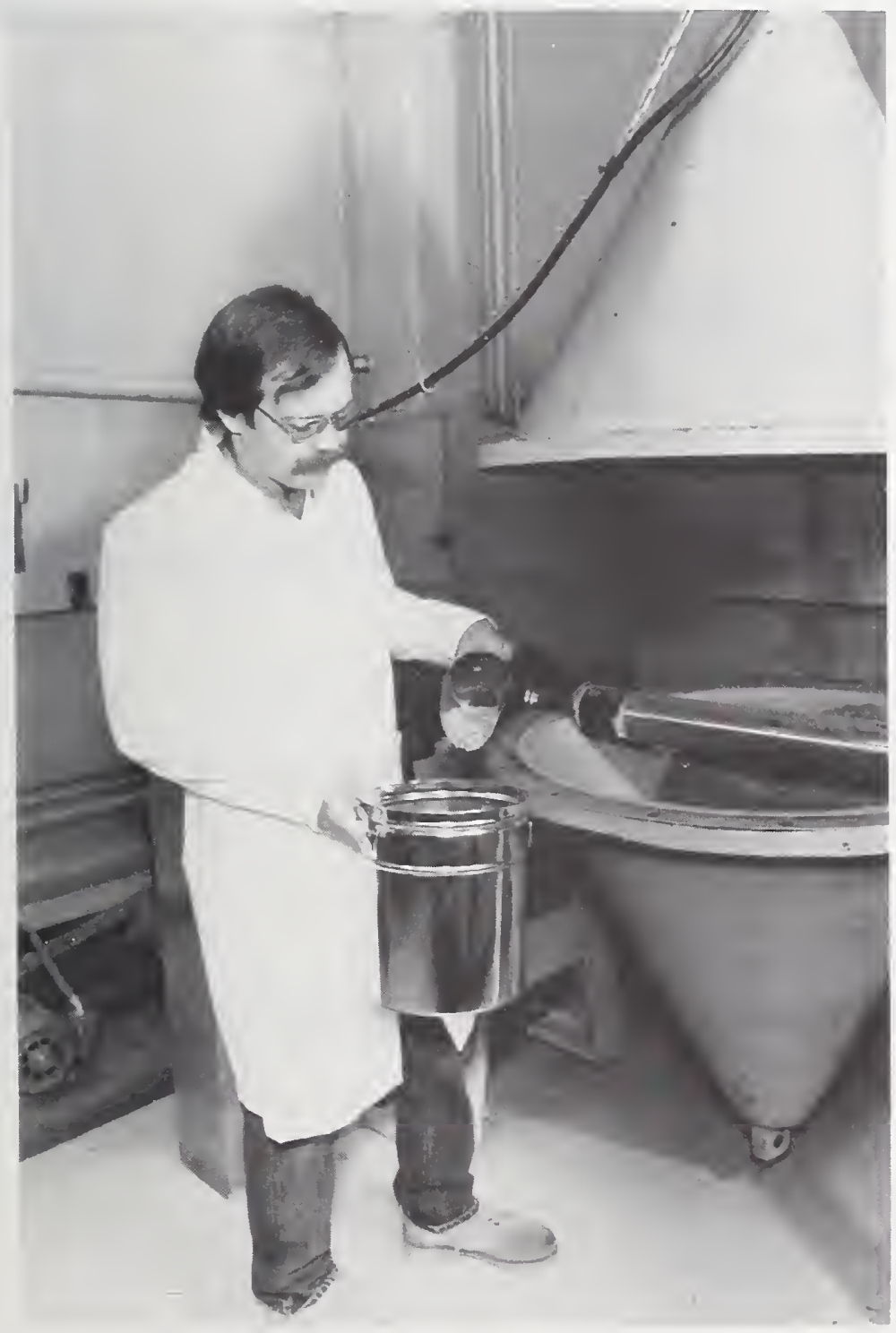

Dale Friend removes some material from the large cone blender for homogeneity analysis. Dale's dedication and availability as supervisor of the preparation and packaging activities is a valued asset of the program. 


\section{High-Purity Metals}

These SRM's are for determining impurity elements in high-purity metals. (See also specific metals.)

\begin{tabular}{|c|c|c|c|c|c|c|c|c|c|c|c|}
\hline \multirow{2}{*}{ SRM } & \multirow{2}{*}{\multicolumn{3}{|c|}{ Type }} & \multirow{2}{*}{\multicolumn{3}{|c|}{ Unit Size }} & \multicolumn{5}{|c|}{$\begin{array}{l}\text { Chemical Composition (Nominal Parts Per } \\
\text { Million by Weight) }\end{array}$} \\
\hline & & & & & & & $\mathrm{Cu}$ & $\mathrm{Ni}$ & $\mathrm{Sn}$ & $\mathbf{P b}$ & $\mathbf{Z r}$ \\
\hline $685 W^{*}$ & \multicolumn{3}{|c|}{ High-Purity Gold (Wire) } & \multirow{2}{*}{\multicolumn{3}{|c|}{$\begin{array}{l}1.4 \mathrm{~mm} \mathrm{D} \times 102 \mathrm{~mm} \text { long } \\
5.9 \mathrm{~mm} \mathrm{D} \times 25 \mathrm{~mm} \text { long }\end{array}$}} & 0.1 & $(<0.05)$ & $(<0.07)$ & & \\
\hline $685 \mathrm{R}^{*}$ & \multicolumn{3}{|c|}{ High-Purity Gold (Rod) } & & & & 0.1 & $(<0.05)$ & $(<0.07)$ & & \\
\hline $680 \mathrm{a}$ & \multicolumn{3}{|c|}{$\begin{array}{l}\text { High-Purity Platinum } \\
\text { (Wire) }\end{array}$} & \multicolumn{3}{|c|}{$\begin{array}{l}0.51 \mathrm{~mm} \mathrm{D}: \mathrm{L} 1(10 \mathrm{~cm}) \\
\mathrm{L} 2(1 \mathrm{~m})\end{array}$} & 0.1 & $<1$ & & $<1$ & $<0.1$ \\
\hline 681 & \multicolumn{3}{|c|}{ Doped-Platinum (Wire) } & \multicolumn{3}{|c|}{$\begin{array}{l}0.51 \mathrm{~mm} \mathrm{D:} \text { L1 }(10 \mathrm{~cm}) \\
\text { L2 }(1 \mathrm{~m})\end{array}$} & 5.0 & 0.5 & & 12 & 11 \\
\hline $682^{*}$ & \multicolumn{3}{|c|}{ High-Purity Zinc } & \multirow{3}{*}{\multicolumn{3}{|c|}{$\begin{array}{l}\text { Semicirc } 57 \mathrm{~mm} \mathrm{D} \\
\text { Semicirc } 57 \mathrm{~mm} \mathrm{D} \\
\text { Shot, } 450 \mathrm{~g}\end{array}$}} & 0.042 & $2(<0.1)$ & $(0.02)$ & & \\
\hline $683^{*}$ & \multirow{2}{*}{\multicolumn{3}{|c|}{$\begin{array}{l}\text { Zinc Metal } \\
\text { Zinc, Intermediate } \\
\text { Purity }\end{array}$}} & & & & 5.9 & & $(0.02)$ & 11.1 & \\
\hline 728 & & & & & & & 5.7 & & $(0.02)$ & 11.1 & \\
\hline 726 & \multicolumn{3}{|c|}{$\begin{array}{l}\text { Selenium, Intermediate } \\
\text { Purity }\end{array}$} & \multicolumn{3}{|c|}{ Shot, $450 \mathrm{~g}$} & $<1$ & & & & \\
\hline $\mathrm{C} 1257$ & \multicolumn{3}{|c|}{ Aluminum, High Purity } & \multicolumn{3}{|c|}{ Disk } & $(<0.1)$ & $(<0.1)$ & $(<0.1)$ & $(<0.1)$ & $(<0.1)$ \\
\hline SRM & Ag & $\mathrm{Mg}$ & In & $\mathrm{Fe}$ & O & Pd & Au & $\mathbf{R h}$ & Ir & $\mathrm{Cd}$ & Tl \\
\hline $685 W^{*}$ & {$[0.1]$} & $(<0.2)$ & $0.00_{7}$ & 0.3 & [2] & & & & & & \\
\hline $685 R^{*}$ & {$[0.1]$} & $(<0.2)$ & $0.00_{7}$ & 0.2 & {$[<2]$} & & & & & & \\
\hline $680 \mathrm{a}$ & $<0.1$ & $<1$ & & 1.3 & 4 & 0.2 & $<1$ & $<0.2$ & $<0.01$ & & \\
\hline 681 & 2.0 & 12 & & 5 & 7 & 6 & 9 & 9 & 11 & & \\
\hline $682^{*}$ & $(0.02)$ & $(<0.1)$ & & $(0.1)$ & $(<0.5)$ & & & & & $(0.1)$ & \\
\hline $683^{*}$ & 1.3 & & & 2.2 & & & & & & 1.1 & $(0.2)$ \\
\hline 728 & 1.1 & & & 2.7 & & & & & & $1.1_{5}$ & $(0.2)$ \\
\hline 726 & $<1$ & $<1$ & S 12 & 1 & $\mathrm{Cr}<1$ & Mo $<0.3$ & $\mathrm{Te} 0.3$ & As $<2$ & $\mathrm{~A} 1<1$ & $\mathbf{B}<1$ & $\mathrm{Ca}<1$ \\
\hline $\mathrm{C} 1257$ & Si 2.0 & 5.0 & & 1.0 & $\mathrm{Cr}(<0.1)$ & & & & & $(<0.1)$ & $\mathrm{Ca}(<0.1)$ \\
\hline
\end{tabular}

*Certificate gives upper limits for other elements found to be present.

Values in parentheses are not certified, but are given for information only.

Values in brackets are subject to greater error since only one method of analysis was employed.

\section{RM 1R-Ultra-Purity Aluminum Polycrystalline Rods}

These rods are intended for use in research on the mechanical and physical properties of extremely pure aluminum; e.g., in the determination of resistivity as a function of strain at cryogenic temperatures to facilitate the design of cryogenic magnets, or superconductor stabilizing elements. Unit of issue: $4.2 \mathrm{~mm}$ in diameter and $25.4 \mathrm{~mm}$ long. 


\section{Microanalytical}

These SRM's provide a highly homogeneous material at microscopic spatial resolution. They are intended primarily for use in calibration of quantitative electron probe, secondary ion mass spectrometry, spark source mass spectrometry, and laser probe microanalytical techniques.

\begin{tabular}{cll}
\hline \multicolumn{1}{c}{ Type } & \\
\hline SRM & \multicolumn{1}{c}{ Unit Size } \\
\hline & & \\
470 & Mineral Glasses (K-411 \& K-412) & 2 Rods: $1 \times 1 \times 15 \mathrm{~mm}$ \\
480 & Tungsten-20\% Mo Alloy & $\begin{array}{l}\text { Rod: } 1 \mathrm{~mm} \mathrm{D,} \mathrm{mm} \text { long } \\
\text { 6 Wire: } 0.5 \mathrm{~mm} \mathrm{D}, 50 \mathrm{~mm} \text { long }\end{array}$ \\
481 & Au-Ag Set & 6 Wire: $0.5 \mathrm{~mm} \mathrm{D}, 50 \mathrm{~mm}$ long \\
\hline 482 & Au-Cu Set & 3 Rods: $2 \times 2 \times 20 \mathrm{~mm}$ \\
1871 & Glasses (K-456, K-493, \& K-523) & 3 Rods: $2 \times 2 \times 20 \mathrm{~mm}$ \\
1872 & Glasses (K-453, K-491, \& K-968) & 3 Rods: $2 \times 2 \times 20 \mathrm{~mm}$ \\
1873 & Glasses (K-458, K-489 \& K-963) & 3 Rods: $2 \times 2 \times 20 \mathrm{~mm}$ \\
1874 & Glasses (K-495, K-490, \& K-546) & 3 Rods: $2 \times 2 \times 20 \mathrm{~mm}$ \\
1875 & Glasses (K-496, K-497, \& K-1013) & 3 mm diameter film \\
\hline 2063 & Thin Film Mg-Si-Ca-Fe & 3 mm diameter film \\
\hline 2064 & Thin Film Al-Si-Ca-Ti-Mn-Zn & \\
\hline
\end{tabular}

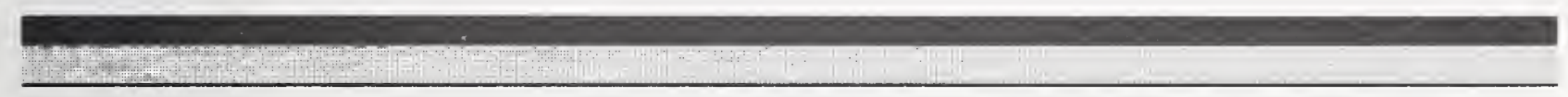

\section{Metals for Microanalysis}

\begin{tabular}{|c|c|c|c|c|c|c|}
\hline \multirow{2}{*}{ SRM } & \multirow{2}{*}{ Type } & \multicolumn{5}{|c|}{$\begin{array}{c}\text { Chemical Composition (Nominal Weight } \\
\text { Percent) }\end{array}$} \\
\hline & & $\mathrm{Au}$ & $\mathrm{Cu}$ & Ag & $\mathbf{W}$ & Mo \\
\hline 480 & Tungsten-20\% Mo Alloy & & & & 78.5 & 21.5 \\
\hline 481 & $\begin{array}{l}\mathrm{Au} 100 \mathrm{~A} \\
\mathrm{Au}-20 \% \mathrm{Ag} \mathrm{B} \\
\mathrm{Au}-40 \% \mathrm{Ag} \mathrm{C} \\
\mathrm{Au}-60 \% \mathrm{Ag} \mathrm{D} \\
\mathrm{Au}-80 \% \mathrm{Ag} \mathrm{E} \\
\mathrm{Ag} 100 \mathrm{~F}\end{array}$ & $\begin{array}{r}100.0_{0} \\
80.0_{5} \\
60.0_{5} \\
40.0_{3} \\
22.4_{3}\end{array}$ & & $\begin{array}{r}19.9_{6} \\
39.9_{2} \\
59.9_{3} \\
77.5_{8} \\
100.0_{0}\end{array}$ & & \\
\hline 482 & $\begin{array}{l}\mathrm{Au} 100 \mathrm{~A} \\
\mathrm{Au}-20 \% \mathrm{Cu} \text { B } \\
\mathrm{Au}-40 \% \mathrm{Cu} \mathrm{C} \\
\mathrm{Au}-60 \% \mathrm{Cu} D \\
\mathrm{Au}-80 \% \mathrm{Cu} \text { E } \\
\mathrm{Cu} 100 \mathrm{~F}\end{array}$ & $\begin{array}{r}100.0_{0} \\
80.1_{5} \\
60.3_{6} \\
40.1_{0} \\
20.1_{2}\end{array}$ & $\begin{array}{r}19.8_{3} \\
39.6_{4} \\
59.9_{2} \\
79.8_{5} \\
100.0_{0}\end{array}$ & & & \\
\hline
\end{tabular}

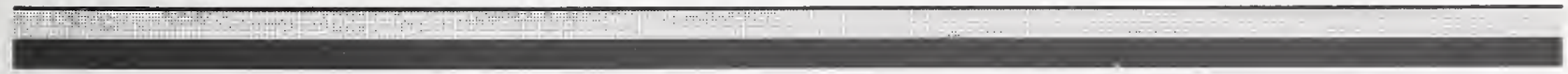




\section{Mineral Glasses for Microanalysis}

\begin{tabular}{|c|c|c|c|c|c|}
\hline $\mathrm{K}-411$ & 54.30 & 14.42 & 14.67 & 15.47 & - \\
\hline $\mathrm{K}-412$ & 45.35 & 9.96 & 19.33 & 15.25 & 9.27 \\
\hline
\end{tabular}

\section{Glasses for Microchemical Analysis}

\begin{tabular}{|c|c|c|c|c|c|c|c|c|c|c|c|c|c|c|c|}
\hline & \multicolumn{3}{|c|}{ SRM 1871} & \multicolumn{3}{|c|}{ SRM 1872} & \multicolumn{3}{|c|}{ SRM 1873} & \multicolumn{3}{|c|}{ SRM 1874} & \multicolumn{3}{|c|}{ SRM 1875} \\
\hline & \multicolumn{3}{|c|}{ Glass } & \multicolumn{3}{|c|}{ Glass } & \multicolumn{3}{|c|}{ Glass } & \multicolumn{3}{|c|}{ Glass } & \multicolumn{3}{|c|}{ Glass } \\
\hline & $K-456$ & $K-493$ & $\mathrm{~K}-523$ & $K-453$ & $K-491$ & $K-968$ & $\mathrm{~K}-\mathbf{4 5 8}$ & $\mathrm{K}-489$ & $K-963$ & $K-495$ & $\mathrm{~K}-490$ & K-546 & $K-496$ & $\mathrm{~K}-497$ & K-1013 \\
\hline \multicolumn{16}{|c|}{ Composition (Nominal Weight Percent) } \\
\hline $\mathrm{Pb}$ & 65.67 & 63.28 & 63.10 & 54.21 & 54.69 & 54.74 & - & $(1.32)$ & - & - & $(1.47)$ & - & - & $(0.86)$ & - \\
\hline $\mathrm{Si}$ & 13.37 & (13.09) & (12.94) & - & $(0.11)$ & - & 23.05 & $(22.23)$ & (21.96) & - & (0.19) & - & - & (0.13) & - \\
\hline $\mathrm{Ge}$ & - & - & $(0.20)$ & 28.43 & 26.10 & 25.93 & - & - & $(0.47)$ & - & - & $(0.50)$ & - & - & $(0.34)$ \\
\hline $\mathrm{Ba}$ & - & - & $(0.61)$ & - & - & $(0.46)$ & 41.79 & 39.53 & 39.21 & - & - & $(0.99)$ & - & - & $(0.52)$ \\
\hline $\mathrm{Zn}$ & - & - & - & - & - & - & 3.01 & 2.93 & 2.95 & - & - & - & - & - & - \\
\hline $\mathbf{P}$ & - & - & $(0.24)$ & - & - & $(0.21)$ & - & - & $(0.33)$ & - & - & $(0.42)$ & 32.98 & 31.59 & 32.26 \\
\hline $\mathrm{Mg}$ & - & - & $(0.12)$ & - & - & $(0.22)$ & - & - & $(0.34)$ & - & - & $(0.17)$ & 6.65 & 6.49 & 5.86 \\
\hline $\mathrm{A} 1$ & - & $(0.13)$ & - & - & $(0.10)$ & - & - & $(0.11)$ & - & 10.89 & $(10.2)$ & $(10.1)$ & 6.47 & 5.97 & 6.08 \\
\hline B & - & - & - & - & - & - & - & - & - & $(23.0)$ & (21.5) & (21.6) & - & {$[0.05]$} & - \\
\hline $\mathrm{Zr}$ & - & $(0.38)$ & $(0.33)$ & - & $(0.26)$ & $(0.48)$ & - & $(0.40)$ & $(0.61)$ & - & $(0.53)$ & $(0.52)$ & - & $(0.34)$ & $(0.45)$ \\
\hline $\mathrm{Ti}$ & - & $(0.20)$ & $(0.21)$ & - & $(0.14)$ & $(0.16)$ & - & $(0.27)$ & $(0.32)$ & - & $(0.31)$ & $(0.39)$ & - & $(0.22)$ & $(0.21)$ \\
\hline $\mathrm{Ce}$ & - & $(0.53)$ & - & - & $(0.59)$ & - & - & {$[0.80]$} & - & - & $(1.46)$ & - & - & $(0.94)$ & - \\
\hline $\mathrm{Ta}$ & - & $(0.64)$ & - & - & $(0.52)$ & - & - & $(0.95)$ & - & - & (1.02) & - & - & $(0.71)$ & - \\
\hline $\mathrm{Fe}$ & - & $(0.25)$ & - & - & $(0.17)$ & - & - & $(0.35)$ & - & - & $(0.38)$ & - & - & $(0.26)$ & - \\
\hline $\mathrm{Li}$ & - & - & - & - & - & - & - & - & - & $(2.3)$ & $(2.2)$ & $(2.2)$ & - & {$[0.0005]$} & - \\
\hline $\mathrm{Ni}$ & - & - & $(0.25)$ & - & - & $(0.20)$ & - & - & $(0.33)$ & - & - & $(0.39)$ & - & - & $(0.31)$ \\
\hline Eu & - & - & $(0.73)$ & - & - & $(0.64)$ & - & - & $(0.95)$ & - & - & $(1.21)$ & - & - & $(0.53)$ \\
\hline $\mathrm{U}$ & - & - & $(0.23)$ & - & - & $(0.05)$ & - & - & $(0.16)$ & - & - & $(0.24)$ & - & - & $(0.15)$ \\
\hline Th & - & - & $(0.08)$ & - & - & $(0.12)$ & - & - & $(0.06)$ & - & - & $(0.16)$ & - & - & $(0.10)$ \\
\hline $\mathrm{Cr}$ & - & - & $(0.20)$ & - & - & $(0.19)$ & - & - & $(0.31)$ & - & - & $(0.14)$ & - & - & $(0.14)$ \\
\hline 0 & $(20.35)$ & $(20.58)$ & $(20.80)$ & (16.73) & (16.45) & (16.67) & (31.86) & $(31.70)$ & $(32.00)$ & (63.49) & $(60.74)$ & (61.36) & $(53.90)^{*}$ & $(52.46)^{\circ}$ & $(53.05)^{*}$ \\
\hline Total & $(99.39)$ & $(99.08)$ & (100.19) & (99.37) & (99.13) & $(100.07)$ & $(99.71)$ & $(100.59)$ & $(100.00)$ & $(99.68)$ & $(100.01)$ & $(100.39)$ & $(100.00)$ & $(100.00)$ & $(100.00)$ \\
\hline
\end{tabular}

Values in parentheses are for information only, they are not certified.

Values in brackets were calculated from the weight of material added to the melt, they are not certified.

* Oxygen values in SRM 1875 were calculated by difference, not by the stoichiometry of the oxides as was done for the other glasses. 


\section{Thin Film for X-Ray Spectrometry}

This SRM is for standardizing chemical analysis by $x$-ray spectrometry and energy loss spectrometry on the analytical electron microscope

\begin{tabular}{ccccccccc}
\hline & & & & & \\
\hline
\end{tabular}

Values in parentheses are not certified, but are given for information only.

Values in

Glass Fibers for Microanalysis-RM 8531

\begin{tabular}{|c|c|c|c|c|c|c|c|c|c|c|}
\hline & K-456 & K-493 & K-453 & K-491 & $\mathrm{K}-\mathbf{4 5 8}$ & K-489 & K-495 & K-490 & K-496 & K-497 \\
\hline \multicolumn{11}{|c|}{ Chemical Composition (Nominal Weight Percent) } \\
\hline $\mathrm{SiO}_{2}$ & 28.77 & 27.89 & - & 0.19 & 49.38 & 46.76 & - & 0.42 & - & 0.27 \\
\hline $\mathrm{PbO}$ & 71.23 & 69.08 & 58.72 & 59.35 & - & 1.28 & - & 1.55 & - & 0.99 \\
\hline $\mathrm{GeO}_{2}$ & - & - & 41.28 & 37.98 & - & - & - & - & - & - \\
\hline $\mathrm{BaO}$ & - & - & - & - & 46.80 & 43.88 & - & - & - & - \\
\hline $\mathrm{ZnO}$ & - & - & - & - & 3.82 & 3.72 & - & - & - & - \\
\hline $\mathrm{P}_{2} \mathrm{O}_{5}$ & - & - & - & - & - & - & - & - & 79.54 & 76.03 \\
\hline $\mathrm{MgO}$ & - & - & 一 & - & - & - & - & - & 9.03 & 8.64 \\
\hline $\mathrm{Al}_{2} \mathrm{O}_{3}$ & - & 0.20 & - & 0.16 & - & 0.29 & 20.00 & 18.68 & 11.43 & 10.92 \\
\hline $\mathrm{B}_{2} \mathrm{O}_{3}$ & - & 0.14 & - & 0.11 & - & 0.20 & 75.00 & 70.00 & - & 0.15 \\
\hline $\mathrm{ZrO}_{2}$ & - & 0.49 & - & 0.40 & 一 & 0.70 & 一 & 0.85 & 一 & 0.54 \\
\hline $\mathrm{TiO}_{2}$ & - & 0.32 & - & 0.26 & - & 0.46 & - & 0.55 & - & 0.35 \\
\hline $\mathrm{CeO}_{2}$ & - & 0.68 & - & 0.56 & - & 0.98 & - & 1.19 & - & 0.76 \\
\hline $\mathrm{Ta}_{2} \mathrm{O}_{5}$ & - & 0.88 & - & 0.72 & - & 1.26 & - & 1.53 & - & 0.98 \\
\hline $\mathrm{Fe}_{2} \mathrm{O}_{3}$ & - & 0.32 & - & 0.26 & - & 0.046 & - & 0.55 & - & 0.35 \\
\hline $\mathrm{Li}_{2} \mathrm{O}$ & - & 0.001 & - & 0.001 & - & 0.002 & 5.00 & 4.67 & - & 0.001 \\
\hline
\end{tabular}




\section{Primary, Working, and Secondary Chemicals}

These SRM's are high-purity chemicals defined as primary, working, and secondary standards in accordance with recommendations of the Analytical Chemistry Section of the International Union of Pure and Applied Chemistry [Ref. Analyst 90, 251 (1965)]. These definitions are as follows:

Primary Standard:

a commercially available substance of purity $100 \pm 0.02$ percent (Purity $99.98+$ percent).

Working Standard:

a commercially available substance of purity $100 \pm 0.05$ percent (Purity $99.95+$ percent).

Secondary Standard:

a substance of lower purity which can be standardized against a primary grade standard.

\begin{tabular}{|c|c|c|c|c|}
\hline SRM & Type & $\begin{array}{l}\text { Wt/Unit } \\
\text { (grams) }\end{array}$ & Certified Use & $\begin{array}{c}\text { Purity } \\
\text { Stoichiometric }\end{array}$ \\
\hline $17 \mathrm{~d}$ & Sucrose & 60 & Polarimetric Value & $(99.9)^{\mathrm{a}}$ \\
\hline $40 \mathrm{~h}$ & Sodium Oxalate & 60 & Reductometric Value & 99.972 \\
\hline $41 c$ & Dextrose (D-Glucose) & 70 & Reductometric Value & 99.9 \\
\hline $83 \mathrm{~d}$ & Arsenic Trioxide & 60 & Reductometric Value & 99.9926 \\
\hline $84 \mathbf{j}$ & Potassium Hydrogen Phthalate & 60 & Acidimetric Value & 99.996 \\
\hline $136 \mathrm{e}$ & Potassium Dichromate & 60 & Oxidimetric Value & 99.984 \\
\hline $350 \mathrm{a}$ & Benzoic Acid & 30 & Acidimetric Value & 99.9958 \\
\hline $723 a$ & Tris(hydroxymethyl)aminomethane & 50 & Basimetric Value & 99.9703 \\
\hline 951 & Boric Acid & 100 & $\begin{array}{l}\text { Acidimetric and Boron Isotopic } \\
\text { Value }\end{array}$ & 100.00 \\
\hline 987 & Strontiuin Carbonate & 1 & Assay and Isotopic & 99.98 \\
\hline 999 & Potassium Chloride & 60 & $\begin{array}{l}\text { Assay Standard for: } \\
\text { Potassium } \\
\text { Chloride }\end{array}$ & $\begin{array}{l}99.98_{1} \\
99.99\end{array}$ \\
\hline
\end{tabular}

${ }^{\text {a Sucrose }}=$ Moisture $<0.02$ percent, Ash $<0.005$ percent.

\section{Microchemical}

\begin{tabular}{|c|c|c|c|c|c|c|c|c|c|c|}
\hline \multirow{2}{*}{ SRM } & \multirow{2}{*}{ Type } & \multirow{2}{*}{$\begin{array}{c}\text { Wt/ } \\
\text { Unit } \\
\text { (grams) }\end{array}$} & \multicolumn{8}{|c|}{ Chemical Composition (Nominal Weight Percent) } \\
\hline & & & C & $\mathbf{H}$ & $\mathbf{N}$ & $\mathbf{B r}$ & $\mathrm{Cl}$ & $\mathbf{F}$ & $\mathbf{S}$ & $\mathrm{CH}_{3} \mathrm{O}-$ \\
\hline $141 \mathrm{c}$ & Acetanilide & 2 & 71.09 & 6.71 & 10.36 & & & & & \\
\hline 142 & Anisic Acid & 2 & & & & & & & & 20.40 \\
\hline $143 c$ & Cystine & 2 & 29.99 & 5.03 & 11.66 & & & & 26.69 & \\
\hline 148 & Nicotinic Acid & 2 & 58.54 & 4.09 & 11.38 & & & & & \\
\hline 2141 & Urea & 2 & & & 46.63 & & & & & \\
\hline 2142 & o-Bromobenzoic Acid & 2 & & & & 39.80 & & & & \\
\hline 2143 & p-Fluorobenzoic Acid & 2 & & & & & & 13.54 & & \\
\hline 2144 & m-Chlorobenzoic Acid & 2 & & & & & 22.62 & & & \\
\hline
\end{tabular}




\section{Spectrometric Solutions}

These SRM's are intended as standard stock solutions for use in atomic absorption spectrometry, optical emission (plasma) spectrometry, or any other analytical technique that requires aqueous solutions for calibrating instruments. Each SRM is a single element solution of $50 \mathrm{~mL}$ with a concentration of $10 \mathrm{mg} / \mathrm{mL}$, except where noted.

\begin{tabular}{|c|c|c|}
\hline SRM & Element & Acid Concentration \\
\hline $\begin{array}{l}3101 \\
3102 \\
3103 \\
3104 \\
3105\end{array}$ & $\begin{array}{l}\text { Aluminum } \\
\text { Antimony } \\
\text { Arsenic } \\
\text { Barium } \\
\text { Beryllium }\end{array}$ & $\begin{array}{l}\mathrm{HCl} 10 \% \\
\mathrm{HCl} 50 \% \\
\mathrm{HCl} 15 \% \\
\mathrm{HCl} 10 \% \\
\mathrm{HCl} 10 \%\end{array}$ \\
\hline $\begin{array}{l}3106 \\
3107 \\
3108 \\
3109 \\
3110\end{array}$ & $\begin{array}{l}\text { Bismuth } \\
\text { Boron (5.00) } \\
\text { Cadmium } \\
\text { Calcuim } \\
\text { Cerium }\end{array}$ & $\begin{array}{l}\mathrm{HNO}_{3} 10 \% \\
\text { Water } \\
\mathrm{HNO}_{3} 10 \% \\
\mathrm{HCl} 10 \% \\
\mathrm{HNO}_{3} 10 \%\end{array}$ \\
\hline $\begin{array}{l}3111 \\
3112 \\
3113 \\
3114 \\
3115\end{array}$ & $\begin{array}{l}\text { Cesium } \\
\text { Chromium } \\
\text { Cobalt } \\
\text { Copper } \\
\text { Dysprosium }\end{array}$ & $\begin{array}{l}\mathrm{HCl} 1 \% \\
\mathrm{HCl} 10 \% \\
\mathrm{HNO}_{3} 10 \% \\
\mathrm{HNO}_{3} 10 \% \\
\mathrm{HCl} 10 \%\end{array}$ \\
\hline $\begin{array}{l}3116 \\
3117 \\
3118 \\
3119 \\
3120\end{array}$ & $\begin{array}{l}\text { Erbium } \\
\text { Europium } \\
\text { Gadolinium } \\
\text { Gallium } \\
\text { Germanium }\end{array}$ & $\begin{array}{l}\mathrm{HCl} 10 \% \\
\mathrm{HCl} 10 \% \\
\mathrm{HCl} 10 \% \\
\mathrm{HCl} 10 \% \\
\text { Oxalic Acid } 10 \%\end{array}$ \\
\hline $\begin{array}{l}3121 \\
3122 \\
3123 \\
3124 \\
3125\end{array}$ & $\begin{array}{l}\text { Gold } \\
\text { Hafnium } \\
\text { Holmium } \\
\text { Indium } \\
\text { Iridium }\end{array}$ & $\begin{array}{l}\mathrm{HC} 110 \% \\
\mathrm{HNO}_{3} 10 \%+\mathrm{HF} 2 \% \\
\mathrm{HC} 110 \% \\
\mathrm{HC} 110 \% \\
\text { IN PREP }\end{array}$ \\
\hline $\begin{array}{l}3126 \\
3127 \\
3128 \\
3129 \\
3130\end{array}$ & $\begin{array}{l}\text { Iron } \\
\text { Lanthanum } \\
\text { Lead } \\
\text { Lithium } \\
\text { Lutetium }\end{array}$ & $\begin{array}{l}\mathrm{HCl} 10 \% \\
\mathrm{HCl} 10 \% \\
\mathrm{HNO}_{3} 10 \% \\
\mathrm{HCl} 1 \% \\
\mathrm{HCl} 10 \%\end{array}$ \\
\hline $\begin{array}{l}3131 \\
3132 \\
3133 \\
3134 \\
3135\end{array}$ & $\begin{array}{l}\text { Magnesium } \\
\text { Manganese } \\
\text { Mercury } \\
\text { Molybdenum } \\
\text { Neodymium }\end{array}$ & $\begin{array}{l}\mathrm{HCl} 10 \% \\
\mathrm{HNO}_{3} 10 \% \\
\mathrm{HNO}_{3} 10 \% \\
\mathrm{HCl} 10 \% \\
\mathrm{HCl} 10 \%\end{array}$ \\
\hline $\begin{array}{l}3136 \\
3137 \\
3138 \\
3139 \\
3140\end{array}$ & $\begin{array}{l}\text { Nickel } \\
\text { Niobium } \\
\text { Palladium } \\
\text { Phosphorus } \\
\text { Platinum }\end{array}$ & $\begin{array}{l}\mathrm{HNO}_{3} 10 \% \\
5 \% \mathrm{HNO}_{3}+2 \% \mathrm{HF} \\
\mathrm{HCl} 10 \% \\
\mathrm{HCl} 0.05 \% \\
\mathrm{HCl} 10 \%\end{array}$ \\
\hline $\begin{array}{l}3141 \\
3142 \\
3143 \\
3144 \\
3145\end{array}$ & $\begin{array}{l}\text { Potassium } \\
\text { Praseodymium } \\
\text { Rhenium } \\
\text { Rhodium } \\
\text { Rubidium }\end{array}$ & $\begin{array}{l}\mathrm{HCl} 1 \% \\
\mathrm{HCl} 10 \% \\
\mathrm{HNO}_{3} 10 \% \\
\text { IN PREP } \\
\mathrm{HCl} 1 \%\end{array}$ \\
\hline
\end{tabular}




\section{Spectrometric Solutions (Continued)}

\begin{tabular}{|c|c|c|}
\hline SRM & Element & Acid Concentration \\
\hline 3146 & Ruthenium & IN PREP \\
\hline 3147 & Samarium & $\mathrm{HC} 110 \%$ \\
\hline 3148 & Scandium & $\mathrm{HCl} 10 \%$ \\
\hline 3149 & Selenium & $\mathrm{HNO}_{3} 10 \%$ \\
\hline 3150 & Silicon & Water \\
\hline 3151 & Silver & $\mathrm{HNO}_{3} 10 \%$ \\
\hline 3152 & Sodium & $\mathrm{HCl} 1 \%$ \\
\hline 3153 & Strontium & HCl $10 \%$ \\
\hline 3154 & Sulfur & $\mathrm{H}_{2} \mathrm{SO}_{4} 0.1 \%$ \\
\hline 3155 & Tantalum & $5 \% \mathrm{HNO}_{3}+2 \% \mathrm{HF}$ \\
\hline 3156 & Tellurium & HC1 10\% \\
\hline 3157 & Terbium & $\mathrm{HCl} 10 \%$ \\
\hline 3158 & Thallium & $\mathrm{HNO}_{3} 10 \%$ \\
\hline 3159 & Thorium & $\mathrm{HNO}_{3} 10 \%$ \\
\hline 3160 & Thulium & $\mathrm{HCl} 10 \%$ \\
\hline 3161 & Tin & $\mathrm{HCl} 60 \%$ \\
\hline 3162 & Titanium & $\mathrm{HCl} 40 \%$ \\
\hline 3163 & Tungsten & $7 \% \mathrm{HNO}_{3}+4 \% \mathrm{HF}$ \\
\hline 3164 & Uranium & $\mathrm{HNO}_{3} 10 \%$ \\
\hline 3165 & Vanadium (5.00) & $\mathrm{HNO}_{3} 10 \%$ \\
\hline 3166 & Ytterbium & HC1 $10 \%$ \\
\hline 3167 & Yttrium & HC1 $10 \%$ \\
\hline 3168 & Zinc & $\mathrm{HCl} 10 \%$ \\
\hline 3169 & Zirconium & $10 \% \mathrm{HNO}_{3}+2 \% \mathrm{HF}$ \\
\hline
\end{tabular}

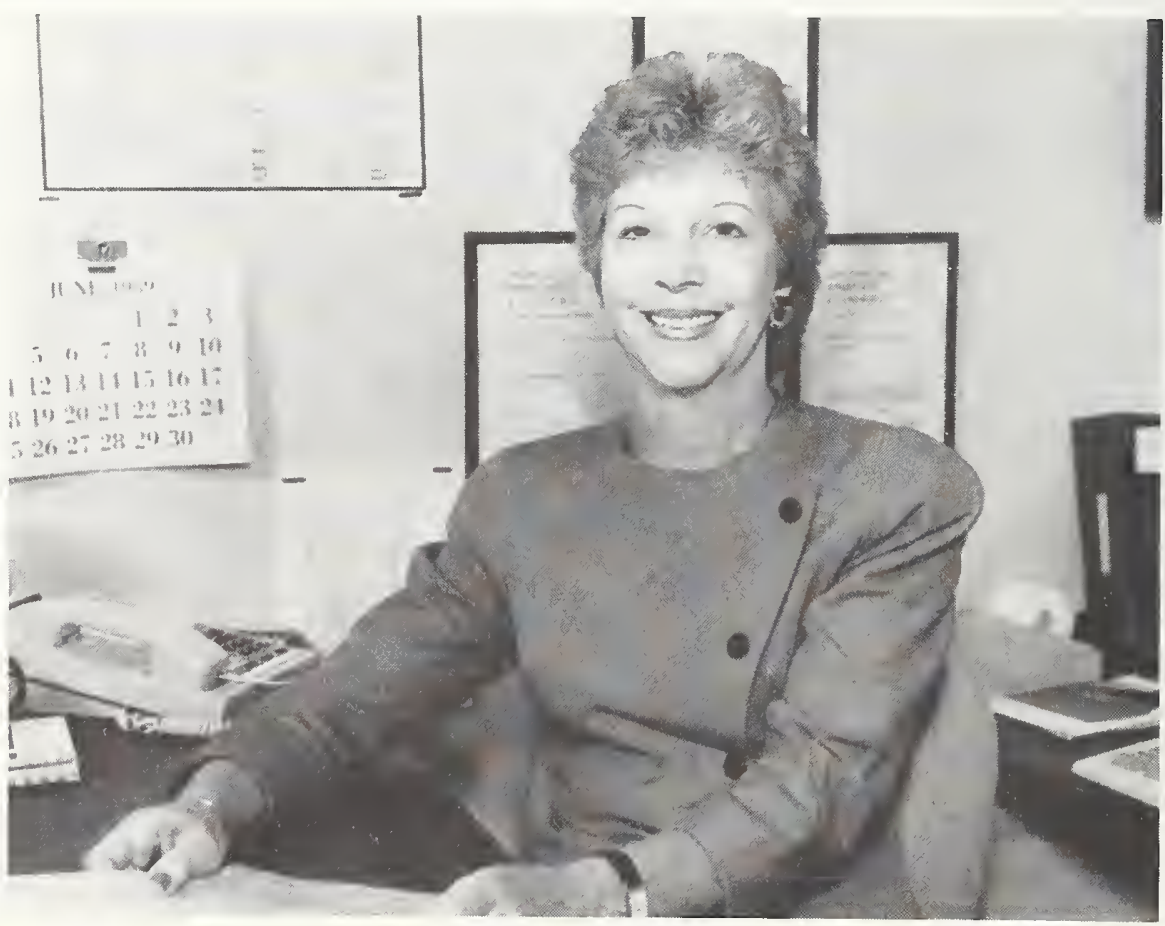

Dolly Downs helps implement the technical and editorial review process for all certificates in addition to performing many other reporting and documentation activities that help keep track of the many certification projects. Her sincerity and neatness are instrumental in keeping all these activities functioning smoothly. 


\section{Multielement Spectrometric Solutions}
Element
Source, Purity
Concentration, $\mu \mathrm{g} / \mathrm{mL}$

SRM 3171 Multielement Mix A Standard Solution

$\begin{array}{ll}\text { Aluminum } & \text { Metal, (99.99+) } \\ \text { Beryllium } & \text { Metal, (99.9) } \\ \text { Cadmium } & \text { Metal, (99.99+) } \\ \text { Chromium } & \text { Metal, (99.99+) } \\ \text { Iron } & \text { Metal, (99.99+) } \\ \text { Magnesium } & \text { Metal, (99.999) } \\ \text { Manganese } & \text { Metal, (99.99) } \\ \text { Nickel } & \text { Metal, (99.999) } \\ \text { Potassium } & \text { KCl, (99.98) } \\ \text { Sodium } & \text { NaCl, }(99.98)\end{array}$

$100.0 \pm 0.5$
$10.0 \pm 0.1$
$100.0 \pm 0.5$
$100.0 \pm 0.5$
$100.0 \pm 0.5$
$100.0 \pm 0.5$
$100.0 \pm 0.5$
$100.0 \pm 0.5$
$500.0 \pm 2.5$
$100.0 \pm 0.5$

SRM 3172 Multielement Mix B Standard Solution

$\begin{array}{ll}\text { Arsenic } & \mathrm{A}_{\mathrm{s} 2} \mathrm{O}_{3},(99.9926) \\ \text { Barium } & \mathrm{BaCO}_{3},(99.99) \\ \text { Calcium } & \mathrm{CaCO}_{3},(99.99) \\ \text { Cobalt } & \mathrm{Metal},(99.99) \\ \text { Copper } & \text { Metal, (99.998) } \\ \text { Lead } & \text { Metal, (99.99) } \\ \text { Selenium } & \text { Metal, (99.99) } \\ \text { Silver } & \mathrm{Metal}^{(99.999+)} \\ \text { Strontium } & \mathrm{SrCO},(99.99) \\ \text { Zinc } & \text { Metal, }(99.99+)\end{array}$

$200.0 \pm 1.0$

$10.0 \pm 0.1$

$10.0 \pm 0.1$

$100.0 \pm 0.5$

$100.0 \pm 0.5$

$100.0 \pm 0.5$

$500.0 \pm 2.5$

$100.0 \pm 0.5$

$10.0 \pm 0.1$

$100.0 \pm 0.5$

SRM 3173 Multielement Mix C Standard Solution

IN PREP

SRM 3174 Multielement Mix D Standard Solution

Aluminum

Beryllium

Boron

Cadmium

Gold

Hafnium

Iron

Lead

Titanium

Zirconium
Metal, $(99.99+)$

Metal, (99.9)

$\mathrm{H}_{3} \mathrm{BO}_{3},(99.99)$

Metal, (99.999)

Metal, (99.999)

Metal, (99.95)

Metal, (99.99)

Metal, (99.995)

Metal, (99.99)

Metal, (99.84)
$100.0 \pm 0.5$

$100.0 \pm 0.5$

$100.0 \pm 0.5$

$100.0 \pm 0.5$

$100.0 \pm 0.5$

$100.0 \pm 0.5$

$100.0 \pm 0.5$

$100.0 \pm 0.5$

$50.0 \pm 0.25$

$100.0 \pm 1.0$ 


\section{Anion Ion Chromatographic Solutions}

These SRM's are single-component solutions prepared gravimetrically for use in anion ion chromatography, or any other technique that requires aqueous standard solutions for calibration on control materials.

\begin{tabular}{clccc}
\hline SRM & Anion & $\begin{array}{c}\text { Wt/Unit } \\
(\mathrm{mL})\end{array}$ & $\begin{array}{c}\text { Concentration } \\
(\mu \mathrm{g} / \mathrm{g})\end{array}$ \\
\hline & & & 50 & 1000 \\
3181 & Sulfate & & 50 & 1000 \\
3182 & Chloride & 50 & 1000 \\
3184 & Fluoride & IN PREP & 50 & \\
3185 & Bromide & IN PREP & 50 & \\
3186 & Nitrate & IN PREP & 50 \\
\hline
\end{tabular}

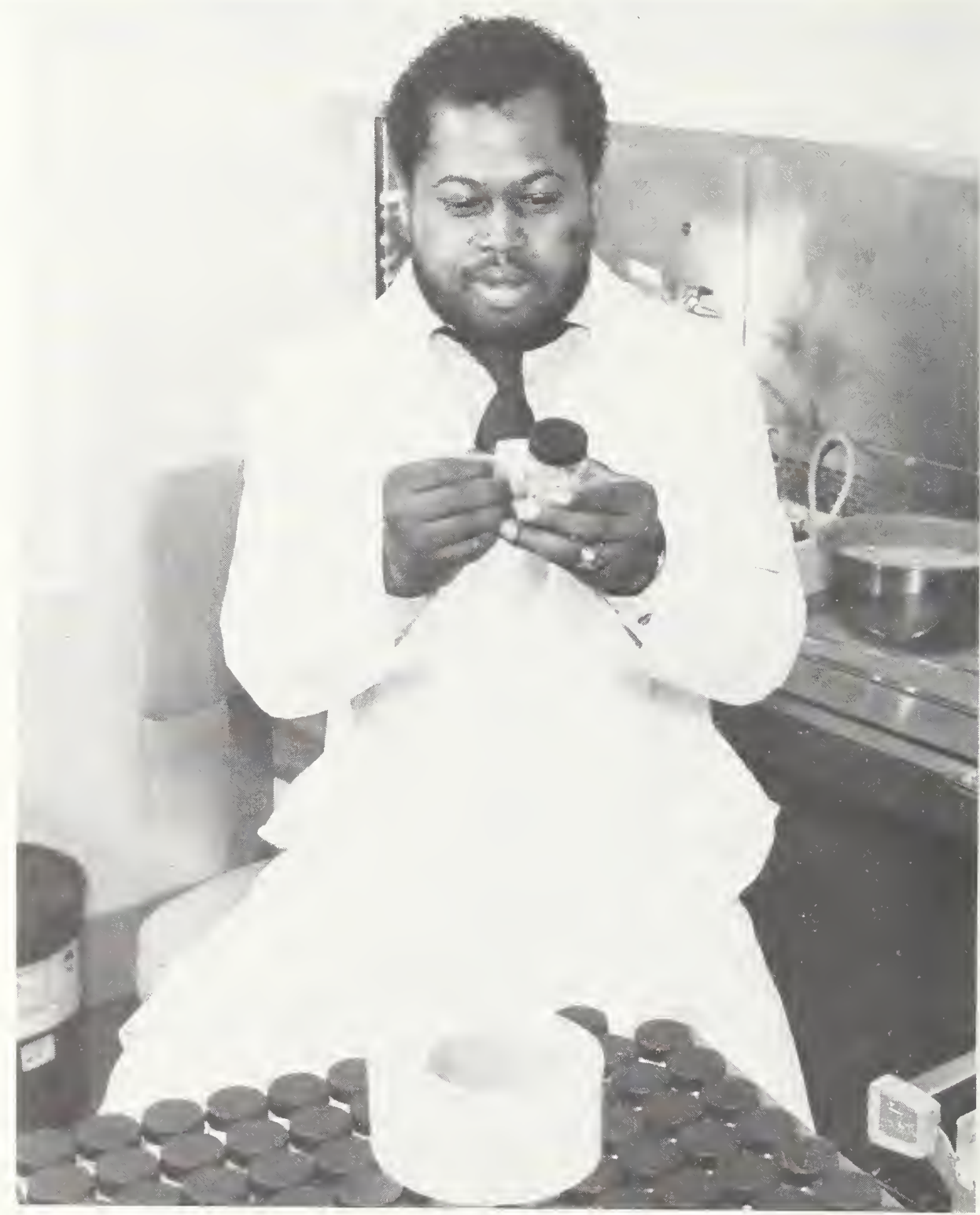

John Savoy, shown here placing labels on bottles of a new SRM, performs his responsibilities in packaging materials with the consistency and orderliness necessary to accurately package and label a large number of different materials. 
Clinical Laboratory

These SRM's are for calibrating apparatus and validating analytical methods used in clinical and pathology laboratories. See also: Spectrophotometric SRM's and Temperature SRM's.

\begin{tabular}{|c|c|c|c|c|}
\hline SRM & Type & $\begin{array}{l}\text { Associated } \\
\text { Publications }\end{array}$ & Purity \% & Wt/Unit \\
\hline 900 & $\begin{array}{l}\text { Antiepilepsy Drug Level Assay (phenyto- } \\
\text { in, ethosuximide, phenobarbital, and pri- } \\
\text { midone) }\end{array}$ & & $\begin{array}{l}4 \text { drugs } \\
3 \text { levels }\end{array}$ & Set of 4 vials \\
\hline 909 & Human Serum & & \# & Set of 6 vials \\
\hline 910 & Sodium Pyruvate & & 98.7 & $25 \mathrm{~g}$ \\
\hline $911 \mathrm{~b}$ & Cholesterol & & 99.8 & $2 \mathrm{~g}$ \\
\hline $912 a$ & Urea & & 99.9 & $25 \mathrm{~g}$ \\
\hline 913 & Uric Acid & \multirow{5}{*}{ SP $260-36$} & 99.7 & $10 \mathrm{~g}$ \\
\hline $914 a$ & Creatinine & & 99.7 & $10 \mathrm{~g}$ \\
\hline 915 & Calcium Carbonate & & 99.9 & $20 \mathrm{~g}$ \\
\hline $916 \mathrm{a}$ & Bilirubin & & 98.3 & $100 \mathrm{mg}$ \\
\hline $917 \mathrm{a}$ & D-Glucose (Dextrose) & & 99.9 & $25 \mathrm{~g}$ \\
\hline 918 & Potassium Chloride & \multirow{5}{*}{$\begin{array}{l}\text { SP } 260-63 \\
\text { SP } 260-60\end{array}$} & 99.9 & $30 \mathrm{~g}$ \\
\hline $919 \mathrm{a}$ & Sodium Chloride & & IN PREP & $30 \mathrm{~g}$ \\
\hline 920 & D-Mannitol & & 99.8 & $50 \mathrm{~g}$ \\
\hline 921 & Cortisol (Hydrocortisone) & & 98.9 & $1 \mathrm{~g}$ \\
\hline 922 & Tris(hydroxymethyl) aminomethane & & 99.99 & $25 \mathrm{~g}$ \\
\hline 923 & Tris(hydroxymethyl) aminomethane $\mathrm{HCl}$ & \multirow{5}{*}{ SP 260-69 } & 99.69 & $35 \mathrm{~g}$ \\
\hline 924 & Lithium Carbonate & & 100.0 & $30 \mathrm{~g}$ \\
\hline 925 & $\begin{array}{l}\text { VMA (4-hydroxy-3-methoxymandelic } \\
\text { acid) }\end{array}$ & & 99.4 & $1 \mathrm{~g}$ \\
\hline 926 & Bovine Serum Albumin (Powder) & & * & $5 \mathrm{~g}$ \\
\hline $927 a$ & Bovine Serum Albumin (7\% Solution) & & * & 10 vials, $2.15 \mathrm{~mL}$ ea. \\
\hline 928 & \multirow{6}{*}{\multicolumn{2}{|c|}{$\begin{array}{l}\text { Lead Nitrate } \\
\text { Magnesium Gluconate Dihydrate } \\
\text { Iron Metal } \\
\text { 4-Nitrophenol } \\
\text { Electrolytes in Serum for ISE } \\
\text { Fat Soluble Vitamins in Human Serum }\end{array}$}} & 100.00 & $30 \mathrm{~g}$ \\
\hline 929 & & & $(100.1)$ & $5 \mathrm{~g}$ \\
\hline 937 & & & 99.90 & $50 \mathrm{~g}$ \\
\hline 938 & & & (99.75) & $15 \mathrm{~g}$ \\
\hline 956 & & & IN PREP & \\
\hline 968 & & & 3 levels & Set of 6 vials \\
\hline 998 & & 94.1 & $0.5 \mathrm{mg}$ \\
\hline 1589 & \multicolumn{2}{|l|}{$\begin{array}{l}\text { Polychlorinated Biphenyls (PCB's) } \\
\text { in Human Serum (as Aroclor 1260) }\end{array}$} & - & Set of 3 bottles \\
\hline 1595 & \multirow{2}{*}{\multicolumn{2}{|c|}{$\begin{array}{l}\text { Tripalmitin } \\
\text { Inorganic Constituents in Bovine Serum }\end{array}$}} & 99.5 & $2 \mathrm{~g}$ \\
\hline 1598 & & & & Set of 2 vials \\
\hline 1599 & \multicolumn{2}{|l|}{$\begin{array}{l}\text { Anticonvulsant Drug Level Assay } \\
\text { (valproic acid and carbamazepine) }\end{array}$} & $\begin{array}{l}2 \text { drugs/ } \\
3 \text { levels }\end{array}$ & Set of 4 vials \\
\hline $1700 \mathrm{a}$ & \multicolumn{2}{|l|}{ Blood Gas: $\mathrm{CO}_{2}-10 \%$, Bal $\mathrm{N}_{2}$} & - & $\begin{array}{l}\text { Cylinder, } 20 \text { cubic } \\
\text { feet }\end{array}$ \\
\hline $1701 \mathrm{a}$ & \multicolumn{2}{|l|}{ Blood Gas: $\mathrm{CO}_{2}-5 \%, \mathrm{O}_{2}-12 \%$, Bal $\mathrm{N}_{2}$} & - & Cylinder, 20 cubic \\
\hline $1702 a$ & \multicolumn{2}{|l|}{ Blood Gas: $\mathrm{CO}_{2}-5 \%, \mathrm{O}_{2}-20 \%$, Bal $\mathrm{N}_{2}$} & - & $\begin{array}{l}\text { Cylinder, } 20 \text { cubic } \\
\text { feet }\end{array}$ \\
\hline $1703 a$ & \multicolumn{2}{|l|}{ Blood Gas: $\mathrm{CO}_{2}-10 \%, \mathrm{O}_{2}-7 \%$, Bal $\mathrm{N}_{2}$} & - & Cylinder, 20 cubic \\
\hline 1951a & \multicolumn{2}{|l|}{ Cholesterol in Human Serum (Frozen) } & IN PREP & Set of 6 bottles \\
\hline $1952 a$ & \multirow{2}{*}{\multicolumn{2}{|c|}{$\begin{array}{l}\text { Cholesterol in Human Serum (Freez } \\
\text { dried) } \\
\text { Aspartate Aminotransferase (AST) } \\
\text { Human Erythrocyte Source }\end{array}$}} & IN PREP & Set of 3 bottles \\
\hline RM 8430 & & & - & Set of 3 bottles \\
\hline
\end{tabular}

*Conforms to NCCLS specification ACC-1.

\# Electrolytes, selected organics. 


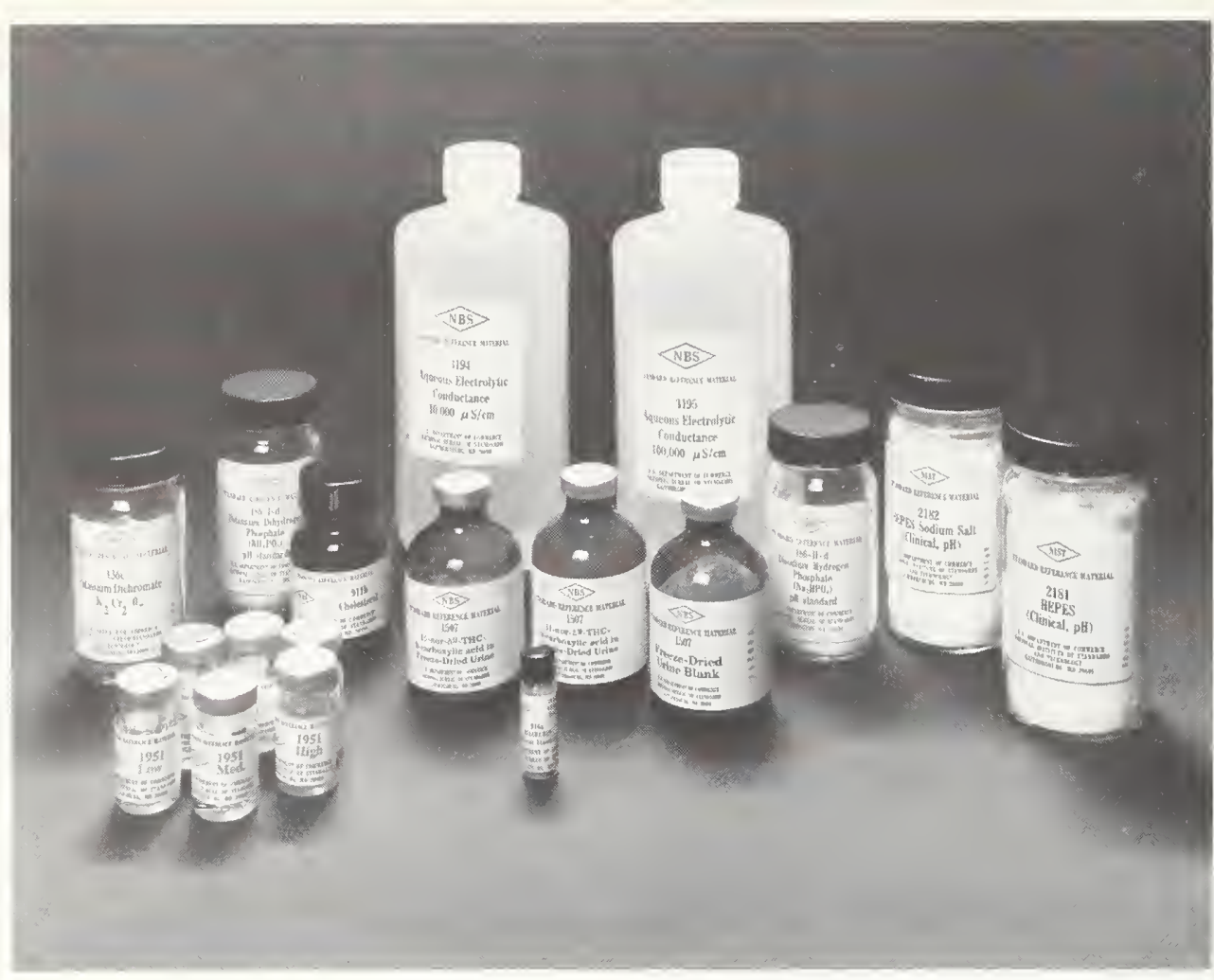

The SRM program has continually expanded in response to needs for certified reference materials. The SRM's shown here have primary relevance to clinical and health related areas of analysis.

\section{Serum Reference Materials}

These materials are for calibrating instrumentation and evaluating the reliability of analytical methods for the determination of major, minor, and trace constituents in blood serum, plasma, and similar biological fluids.

\section{Concentrations}

\section{Constituent}

SRM 909 (Procedure A)

SRM 909 (Procedure B)

\begin{tabular}{|c|c|c|c|c|}
\hline Cadmium & 1.46 & $\mathrm{ng} / \mathrm{mL} / \mathrm{g}$ & 1.24 & $\mathrm{ng} / \mathrm{mL}$ \\
\hline Calcium & 3.560 & $\mathrm{mmo} 1 / \mathrm{L} / \mathrm{g}$ & 3.013 & $\mathrm{mmo} 1 / \mathrm{L}$ \\
\hline Chloride & 128.0 & $\mathrm{mmol} / \mathrm{L} / \mathrm{g}$ & 108.4 & $\mathrm{mmo} 1 / \mathrm{L}$ \\
\hline Chromium & 108 & $\mathrm{ng} / \mathrm{mL} / \mathrm{g}$ & 91.3 & $\mathrm{ng} / \mathrm{mL}$ \\
\hline Cholesterol & 4.326 & $\mathrm{mmol} / \mathrm{L} / \mathrm{g}$ & 3.66 & $\mathrm{mmo} 1 / \mathrm{L}$ \\
\hline Copper & 1.29 & $\mu \mathrm{g} / \mathrm{mL} / \mathrm{g}$ & 1.10 & $\mu \mathrm{g} / \mathrm{mL}$ \\
\hline Creatinine & 0.179 & $\mathrm{mmol} / \mathrm{L} / \mathrm{g}$ & 0.152 & $\mathrm{mmol} / \mathrm{L}$ \\
\hline Glucose & 7.38 & $\mathrm{mmol} / \mathrm{L} / \mathrm{g}$ & 6.25 & $\mathrm{mmol} / \mathrm{L}$ \\
\hline Iron & 2.34 & $\mu \mathrm{g} / \mathrm{mL} / \mathrm{g}$ & 1.98 & $\mu \mathrm{g} / \mathrm{mL}$ \\
\hline Lead & 23.7 & $\mathrm{ng} / \mathrm{mL} / \mathrm{g}$ & 20.0 & $\mathrm{ng} / \mathrm{mL}$ \\
\hline Lithium & 1.945 & $\mathrm{mmol} / \mathrm{L} / \mathrm{g}$ & 1.65 & $\mathrm{mmol} / \mathrm{L}$ \\
\hline Magnesium & 1.425 & $\mathrm{mmol} / \mathrm{L} / \mathrm{g}$ & 1.21 & $\mathrm{mmol} / \mathrm{L}$ \\
\hline Potassium & 4.155 & $\mathrm{mmol} / \mathrm{L} / \mathrm{g}$ & 3.52 & $\mathrm{mmol} / \mathrm{L}$ \\
\hline Sodium & 158.4 & $\mathrm{mmol} / \mathrm{L} / \mathrm{g}$ & 134.1 & $\mathrm{mmol} / \mathrm{L}$ \\
\hline Urea & 11.39 & $\mathrm{mmol} / \mathrm{L} / \mathrm{g}$ & 9.64 & $\mathrm{mmol} / \mathrm{L}$ \\
\hline Uric Acid & 0.567 & $\mathrm{mmol} / \mathrm{L} / \mathrm{g}$ & 0.480 & $\mathrm{mmol} / \mathrm{L}$ \\
\hline Vanadium & 3.19 & $\mathrm{ng} / \mathrm{mL} / \mathrm{g}$ & 2.70 & $\mathrm{ng} / \mathrm{mL}$ \\
\hline
\end{tabular}




\section{Biological Materials}

These SRM's are intended for use in the calibration of apparatus and methods used in the analysis of biological materials for major, minor, and trace constituents.

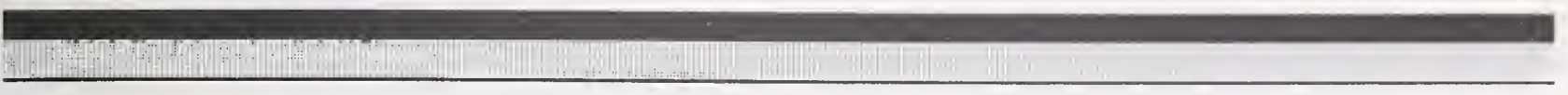

\section{Food and Beverage}

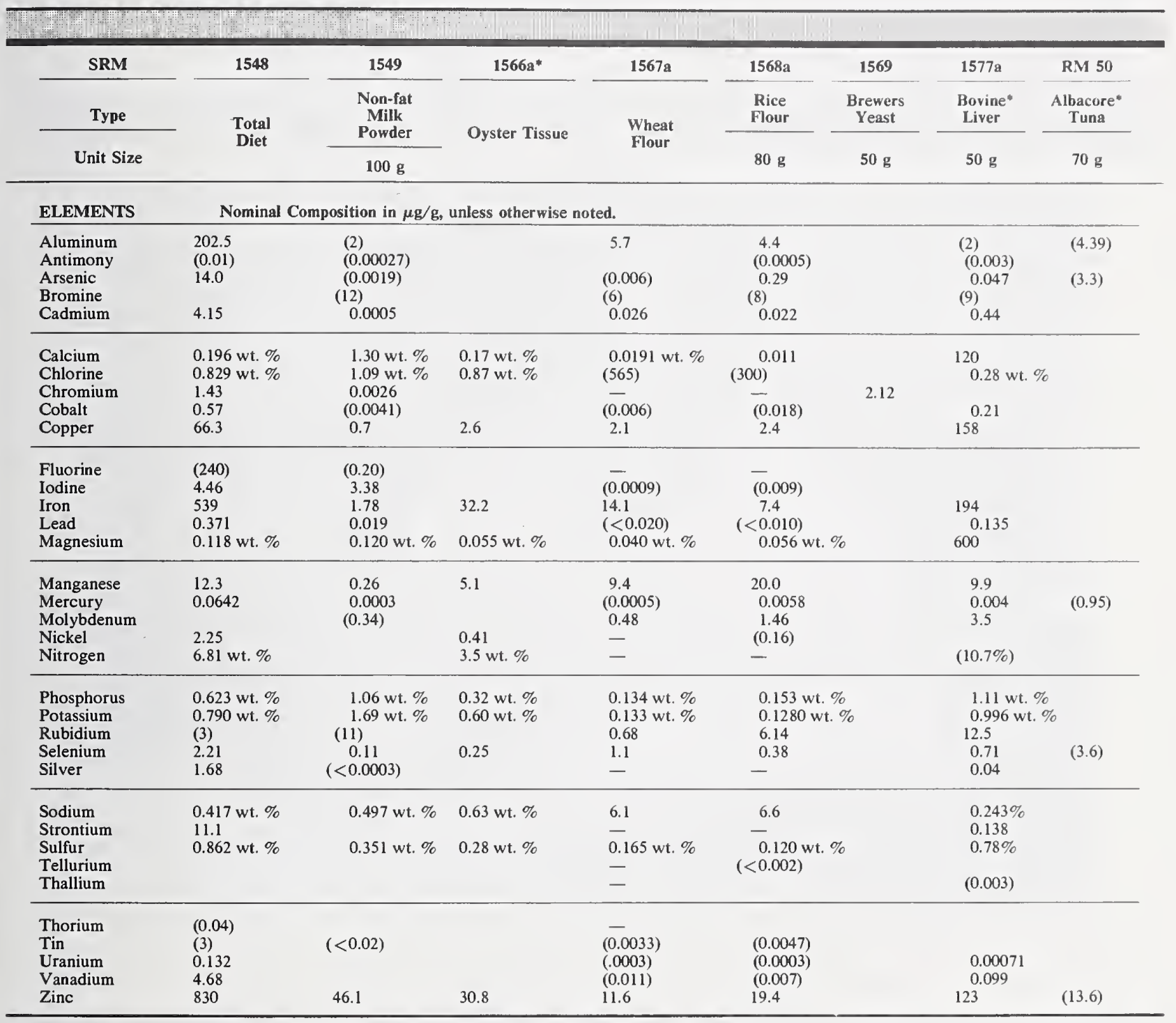

Values in parentheses are not certified, but are given for information only.

*Indicates freeze-dried. 


\section{Food and Beverage (Continued)}

SRM $\quad 1845$

Type Cholesterol in Whole Egg Powder

Unit Size 3 bottles, $8 \mathrm{~g}$ each

Cholesterol $19.0 \mathrm{mg} / \mathrm{g}$
1563

Cholesterol and Fat Soluble Vitamins in Coconut Oil

Set of 10

IN PREP

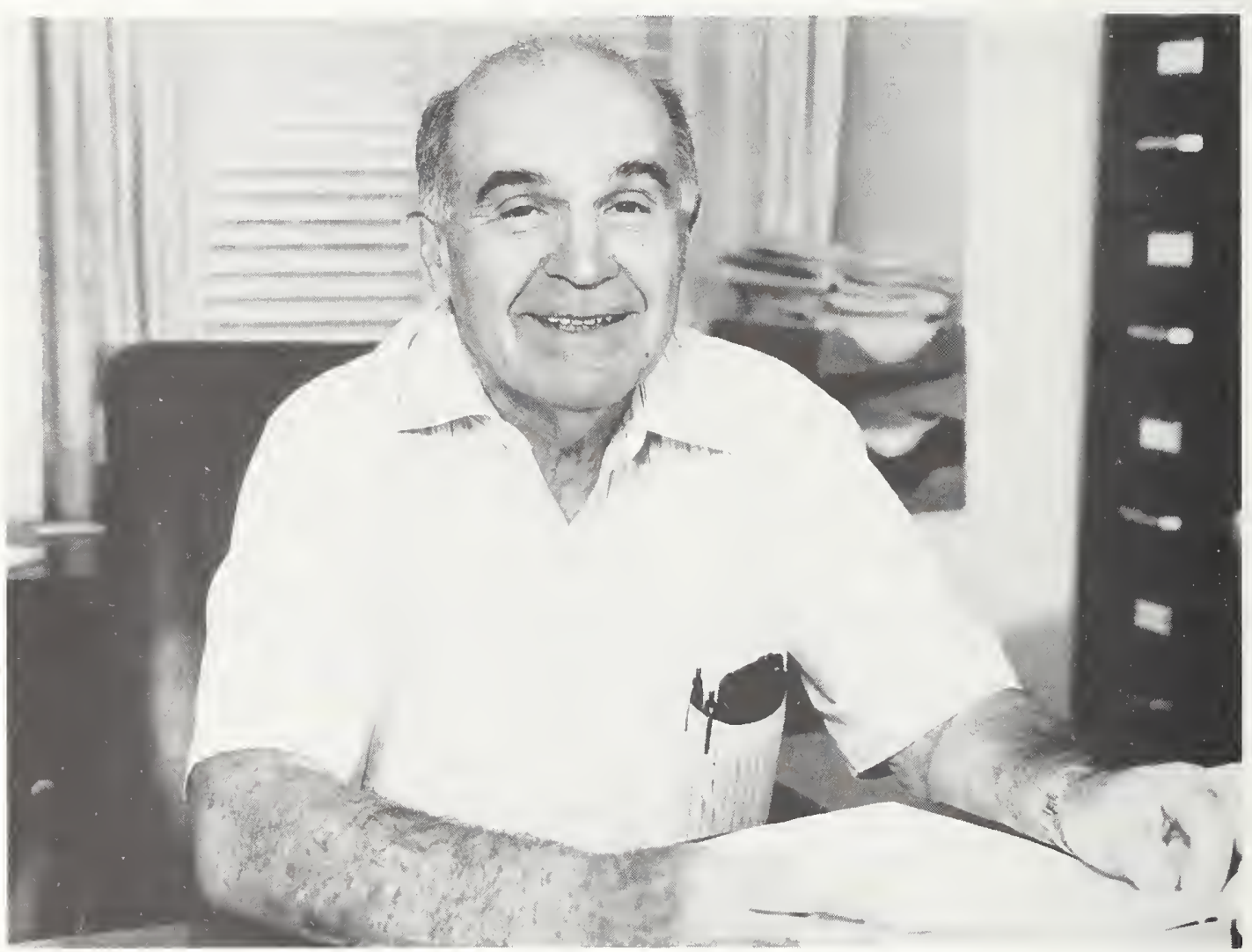

Bob Alvarez, a project manager for OSRM, friendly and outgoing, provides a rich background of experience and knowledge in the SRM program. Bob provides leadership for many of the organic, clinical, chemical and environmental SRM's.

\section{Ethanol Solutions See also: Alcohol in Reference Fuels}

\begin{tabular}{cllcc}
\hline & Type & Certified Constituent & Wt/Unit \\
\hline \multirow{2}{*}{ SRM } & & & & Set of 10, 10-mL vials \\
1590 & Stabilized Wine & Ethanol: $18.57 \%$ by volume & Set: $1,15-\mathrm{mL}$ vial & $2,3-\mathrm{mL}$ vials \\
& Ethanol-Water & Ethanol: 95.629 wt\% & 3-mL vials \\
\hline
\end{tabular}




\section{Agricultural}

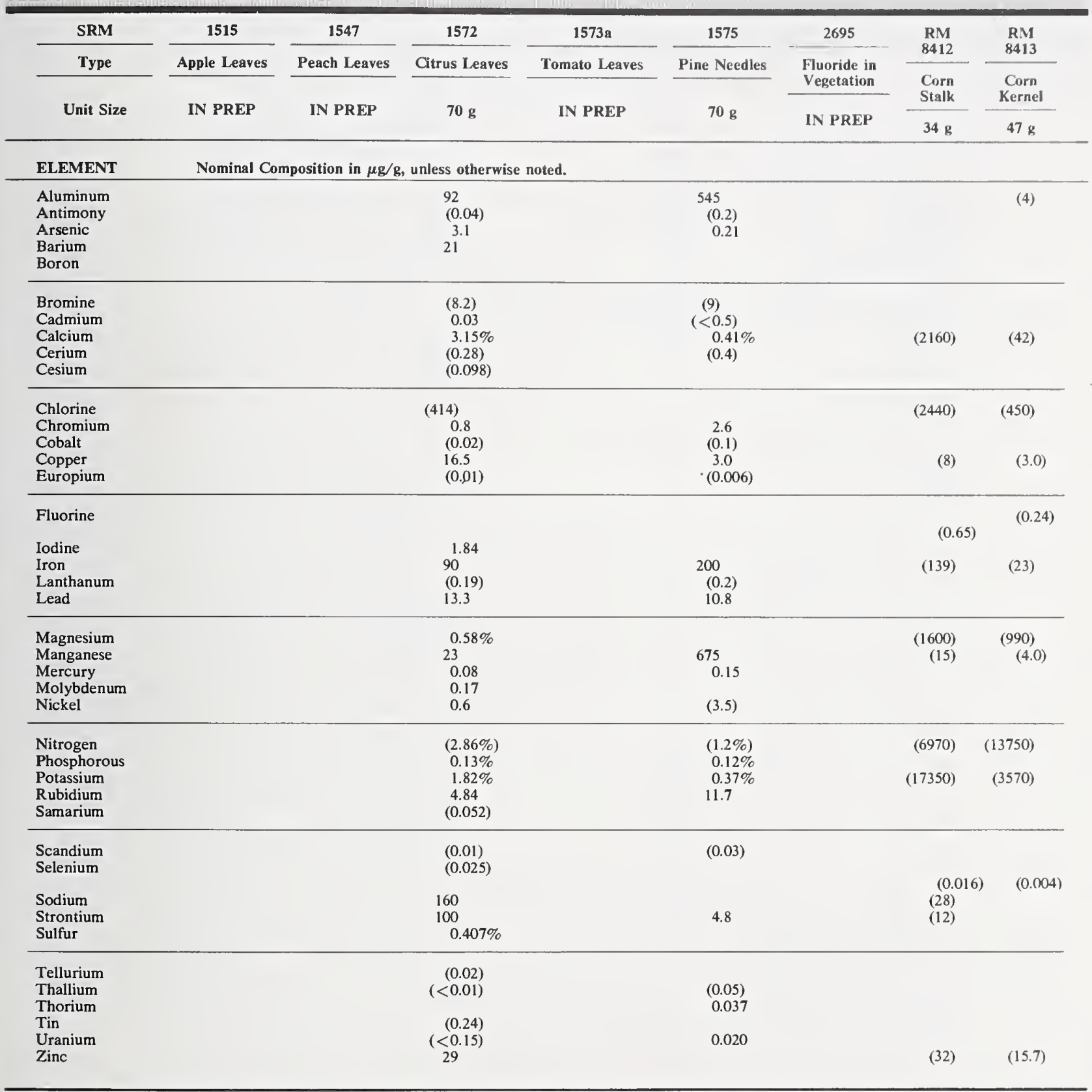

Values in parentheses are not certified, but are given for information only. 


\section{Environmental Materials}

\section{Analyzed Gases}

These SRM's are for calibrating apparatus used to measure various components of gas mixtures and atmospheric pollutants. All cylinders conform to the appropriate DOT specifications.

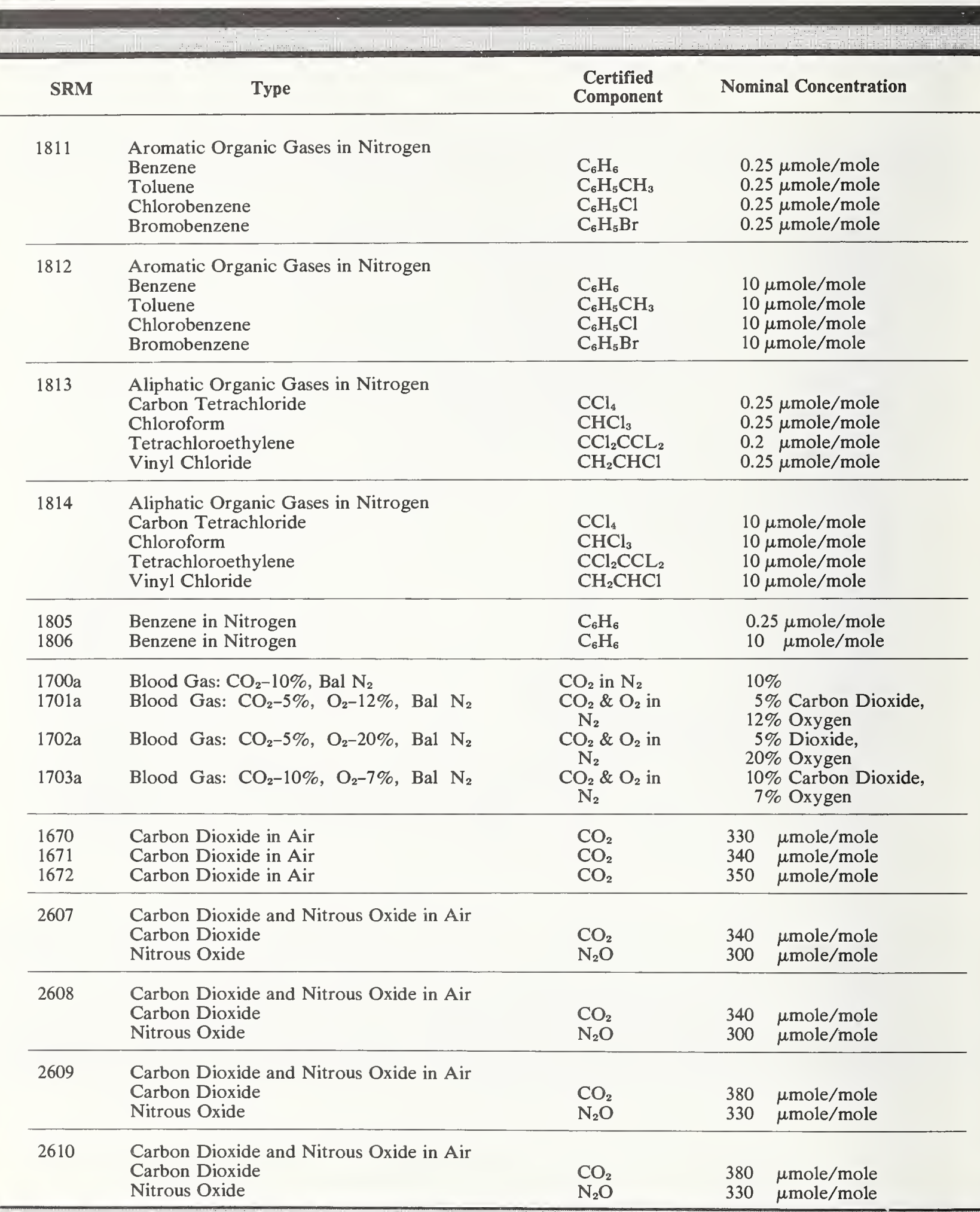




\section{Analyzed Gases (Continued)}

\begin{tabular}{|c|c|c|c|c|}
\hline SRM & Type & $\begin{array}{c}\text { Certified } \\
\text { Component }\end{array}$ & Nom & Concentration \\
\hline $\begin{array}{l}1674 b \\
1675 b \\
2619 a \\
2620 a \\
2621 a \\
2622 a \\
2623 a \\
2624 a \\
2625 a \\
2626 a\end{array}$ & $\begin{array}{l}\text { Carbon Dioxide in Nitrogen } \\
\text { Carbon Dioxide in Nitrogen } \\
\text { Carbon Dioxide in Nitrogen } \\
\text { Carbon Dioxide in Nitrogen } \\
\text { Carbon Dioxide in Nitrogen } \\
\text { Carbon Dioxide in Nitrogen } \\
\text { Carbon Dioxide in Nitrogen } \\
\text { Carbon Dioxide in Nitrogen } \\
\text { Carbon Dioxide in Nitrogen } \\
\text { Carbon Dioxide in Nitrogen }\end{array}$ & $\begin{array}{l}\mathrm{CO}_{2} \\
\mathrm{CO}_{2} \\
\mathrm{CO}_{2} \\
\mathrm{CO}_{2} \\
\mathrm{CO}_{2} \\
\mathrm{CO}_{2} \\
\mathrm{CO}_{2} \\
\mathrm{CO}_{2} \\
\mathrm{CO}_{2} \\
\mathrm{CO}_{2}\end{array}$ & $\begin{array}{r}7.0 \\
14.0 \\
0.5 \\
1.0 \\
1.5 \\
2.0 \\
2.5 \\
3.0 \\
3.5 \\
4.0\end{array}$ & $\begin{array}{l}\text { mole percent } \\
\text { mole percent } \\
\text { mole percent } \\
\text { mole percent } \\
\text { mole percent } \\
\text { mole percent } \\
\text { mole percent } \\
\text { mole percent } \\
\text { mole percent } \\
\text { mole percent }\end{array}$ \\
\hline $\begin{array}{l}2612 a \\
2613 a \\
2614 a\end{array}$ & $\begin{array}{l}\text { Carbon Monoxide in Air } \\
\text { Carbon Monoxide in Air } \\
\text { Carbon Monoxide in Air }\end{array}$ & $\begin{array}{l}\mathrm{CO} \\
\mathrm{CO} \\
\mathrm{CO}\end{array}$ & $\begin{array}{l}10 \\
20 \\
45\end{array}$ & $\begin{array}{l}\mu \mathrm{mole} / \mathrm{mole} \\
\mu \mathrm{mole} / \mathrm{mole} \\
\mu \mathrm{mole} / \mathrm{mole}\end{array}$ \\
\hline $\begin{array}{l}1677 c \\
1678 c \\
1679 c \\
1680 b \\
1681 b\end{array}$ & $\begin{array}{l}\text { Carbon Monoxide in Nitrogen } \\
\text { Carbon Monoxide in Nitrogen } \\
\text { Carbon Monoxide in Nitrogen } \\
\text { Carbon Monoxide in Nitrogen } \\
\text { Carbon Monoxide in Nitrogen }\end{array}$ & $\begin{array}{l}\mathrm{CO} \\
\mathrm{CO} \\
\mathrm{CO} \\
\mathrm{CO} \\
\mathrm{CO}\end{array}$ & $\begin{array}{r}10 \\
50 \\
100 \\
500 \\
1000\end{array}$ & $\begin{array}{l}\mu \text { mole } / \text { mole } \\
\mu \text { mole } / \text { mole } \\
\mu \text { mole } / \text { mole } \\
\mu \text { mole } / \text { mole } \\
\mu \text { mole } / \text { mole }\end{array}$ \\
\hline $\begin{array}{l}2635 a \\
2636 a \\
2637 a \\
2638 a \\
2639 a\end{array}$ & $\begin{array}{l}\text { Carbon Monoxide in Nitrogen } \\
\text { Carbon Monoxide in Nitrogen } \\
\text { Carbon Monoxide in Nitrogen } \\
\text { Carbon Monoxide in Nitrogen } \\
\text { Carbon Monoxide in Nitrogen }\end{array}$ & $\begin{array}{l}\mathrm{CO} \\
\mathrm{CO} \\
\mathrm{CO} \\
\mathrm{CO} \\
\mathrm{CO}\end{array}$ & $\begin{array}{r}25 \\
250 \\
2500 \\
5000 \\
1\end{array}$ & $\begin{array}{l}\mu \mathrm{mole} / \mathrm{mole} \\
\mu \mathrm{mole} / \mathrm{mole} \\
\mu \mathrm{mole} / \mathrm{mole} \\
\mu \mathrm{mole} / \mathrm{mole} \\
\text { mole percent }\end{array}$ \\
\hline $\begin{array}{l}2640 \\
2641 \\
2642 a \\
2725 \\
2726 \\
2727 \\
\\
2728\end{array}$ & $\begin{array}{l}\text { Carbon Monoxide in Nitrogen } \\
\text { Carbon Monoxide in Nitrogen } \\
\text { Carbon Monoxide in Nitrogen } \\
\text { Carbon Monoxide and Propane in } \\
\text { Nitrogen } \\
\text { Carbon Monoxide and Propane in } \\
\text { Nitrogen } \\
\text { Carbon Monoxide, Propane and } \\
\text { Carbon Dioxide in Nitrogen } \\
\text { Carbon Monoxide, Propane and } \\
\text { Carbon Dioxide in Nitrogen }\end{array}$ & $\begin{array}{l}\mathrm{CO} \\
\mathrm{CO} \\
\mathrm{CO} \\
\mathrm{CO} \\
\mathrm{C}_{3} \mathrm{H}_{8} \\
\mathrm{CO} \\
\mathrm{C}_{3} \mathrm{H}_{8} \\
\mathrm{CO} \\
\mathrm{C}_{3} \mathrm{H}_{8} \\
\mathrm{CO}_{2} \\
\mathrm{CO} \\
\mathrm{C}_{3} \mathrm{H}_{8} \\
\mathrm{CO}_{2}\end{array}$ & $\begin{array}{c}2 \\
4 \\
8 \\
1.6 \\
600 \\
8 \\
3000 \\
1.6 \\
600 \\
11 \\
8 \\
3000 \\
14\end{array}$ & $\begin{array}{l}\text { mole percent } \\
\text { mole percent } \\
\text { mole percent } \\
\text { mole percent } \\
\mu \text { mole/mole } \\
\text { mole percent } \\
\mu \text { mole/mole } \\
\text { mole percent } \\
\mu \text { mole/mole } \\
\text { mole percent } \\
\text { mole percent } \\
\mu \text { mole/mole } \\
\text { mole percent }\end{array}$ \\
\hline $\begin{array}{l}1658 \mathrm{a} \\
1659 \mathrm{a} \\
1660 \mathrm{a}\end{array}$ & $\begin{array}{l}\text { Methane in Air } \\
\text { Methane in Air } \\
\text { Methane-Propane in Air }\end{array}$ & $\begin{array}{l}\mathrm{CH}_{4} \\
\mathrm{CH}_{4} \\
\mathrm{CH}_{4} \\
\mathrm{C}_{3} \mathrm{H}_{8}\end{array}$ & $\begin{array}{r}1 \\
10 \\
4 \\
1\end{array}$ & $\begin{array}{l}\mu \mathrm{mole} / \mathrm{mole} \\
\mu \mathrm{mole} / \mathrm{mole} \\
\mu \mathrm{mole} / \mathrm{mole} \\
\mu \mathrm{mole} / \mathrm{mole}\end{array}$ \\
\hline $\begin{array}{l}1683 \mathrm{~b} \\
1684 \mathrm{~b} \\
1685 \mathrm{~b} \\
1686 \mathrm{~b} \\
1687 \mathrm{~b}\end{array}$ & $\begin{array}{l}\text { Nitric Oxide in Nitrogen } \\
\text { Nitric Oxide in Nitrogen } \\
\text { Nitric Oxide in Nitrogen } \\
\text { Nitric Oxide in Nitrogen } \\
\text { Nitric Oxide in Nitrogen }\end{array}$ & $\begin{array}{l}\text { NO } \\
\text { NO } \\
\text { NO } \\
\text { NO } \\
\text { NO }\end{array}$ & $\begin{array}{r}50 \\
100 \\
250 \\
500 \\
1000\end{array}$ & $\begin{array}{l}\mu \mathrm{mole} / \mathrm{mole} \\
\mu \mathrm{mole} / \mathrm{mole} \\
\mu \mathrm{mole} / \mathrm{mole} \\
\mu \mathrm{mole} / \mathrm{mole} \\
\mu \mathrm{mole} / \mathrm{mole}\end{array}$ \\
\hline $\begin{array}{l}2627 a \\
2628 a \\
2629 a\end{array}$ & $\begin{array}{l}\text { Nitric Oxide in Nitrogen } \\
\text { Nitric Oxide in Nitrogen } \\
\text { Nitric Oxide in Nitrogen }\end{array}$ & $\begin{array}{l}\text { NO } \\
\text { NO } \\
\text { NO }\end{array}$ & $\begin{array}{r}5 \\
10 \\
20\end{array}$ & $\begin{array}{l}\mu \mathrm{mole} / \mathrm{mole} \\
\mu \mathrm{mole} / \mathrm{mole} \\
\mu \mathrm{mole} / \mathrm{mole}\end{array}$ \\
\hline
\end{tabular}




\section{Analyzed Gases (Continued)}

\begin{tabular}{|c|c|c|c|c|}
\hline SRM & Type & $\begin{array}{c}\text { Certified } \\
\text { Component }\end{array}$ & \multicolumn{2}{|c|}{ Nominal Concentration } \\
\hline 2630 & Nitric Oxide in Nitrogen & NO & 1500 & $\mu \mathrm{mole} / \mathrm{mole}$ \\
\hline 2631 & Nitric Oxide in Nitrogen & NO & 3000 & $\mu \mathrm{mole} / \mathrm{mole}$ \\
\hline 2654 & Nitrogen Dioxide in Air & $\mathrm{NO}_{2}$ & 500 & $\mu \mathrm{mole} / \mathrm{mole}$ \\
\hline 2655 & Nitrogen Dioxide in Air & $\mathrm{NO}_{2}$ & 1000 & $\mu \mathrm{mole} / \mathrm{mole}$ \\
\hline 2656 & Nitrogen Dioxide in Air & $\mathrm{NO}_{2}$ & 2500 & $\mu \mathrm{mole} / \mathrm{mole}$ \\
\hline $2657 a$ & Oxygen in Nitrogen & $\mathrm{O}_{2}$ & 2 & mole percent \\
\hline $2658 \mathrm{a}$ & Oxygen in Nitrogen & $\mathrm{O}_{2}$ & 10 & mole percent \\
\hline $2659 a$ & Oxygen in Nitrogen & $\mathrm{O}_{2}$ & 21 & mole percent \\
\hline $1665 \mathrm{~b}$ & Propane in Air & $\mathrm{C}_{3} \mathrm{H}_{8}$ & 3 & $\mu \mathrm{mole} / \mathrm{mole}$ \\
\hline $1666 \mathrm{~b}$ & Propane in Air & $\mathrm{C}_{3} \mathrm{H}_{8}$ & 10 & $\mu \mathrm{mole} / \mathrm{mole}$ \\
\hline $1667 \mathrm{~b}$ & Propane in Air & $\mathrm{C}_{3} \mathrm{H}_{8}$ & 50 & $\mu \mathrm{mole} / \mathrm{mole}$ \\
\hline $1668 b$ & Propane in Air & $\mathrm{C}_{3} \mathrm{H}_{8}$ & 100 & $\mu \mathrm{mole} / \mathrm{mole}$ \\
\hline $1669 b$ & Propane in Air & $\mathrm{C}_{3} \mathrm{H}_{8}$ & 500 & $\mu \mathrm{mole} / \mathrm{mole}$ \\
\hline $2645 a$ & Propane in Nitrogen & $\mathrm{C}_{3} \mathrm{H}_{8}$ & 500 & $\mu \mathrm{mole} / \mathrm{mole}$ \\
\hline $2646 a$ & Propane in Nitrogen & $\mathrm{C}_{3} \mathrm{H}_{8}$ & 1000 & $\mu \mathrm{mole} / \mathrm{mole}$ \\
\hline $2647 a$ & Propane in Nitrogen & $\mathrm{C}_{3} \mathrm{H}_{8}$ & 2500 & $\mu \mathrm{mole} / \mathrm{mole}$ \\
\hline $2648 a$ & Propane in Nitrogen & $\mathrm{C}_{3} \mathrm{H}_{8}$ & 5000 & $\mu \mathrm{mole} / \mathrm{mole}$ \\
\hline 2649 & Propane in Nitrogen & $\mathrm{C}_{3} \mathrm{H}_{8}$ & 1 & mole percent \\
\hline 2650 & Propane in Nitrogen & $\mathrm{C}_{3} \mathrm{H}_{8}$ & 2 & mole percent \\
\hline 2651 & $\begin{array}{l}\text { Propane in Nitrogen and } \\
\text { Oxygen }\end{array}$ & $\mathrm{C}_{3} \mathrm{H}_{8}$ & $\begin{array}{l}0.01 \\
50\end{array}$ & mole percent \\
\hline \multirow[t]{2}{*}{2652} & Propane in Nitrogen and & $\mathrm{C}_{3} \mathrm{H}_{8}$ & 0.01 & mole percent \\
\hline & Oxygen & $\mathrm{O}_{2}$ & 10.0 & mole percent \\
\hline $1661 \mathrm{a}$ & Sulfur Dioxide in Nitrogen & $\mathrm{SO}_{2}$ & 500 & $\mu \mathrm{mole} / \mathrm{mole}$ \\
\hline $1662 a$ & Sulfur Dioxide in Nitrogen & $\mathrm{SO}_{2}$ & 1000 & $\mu \mathrm{mole} / \mathrm{mole}$ \\
\hline $1663 a$ & Sulfur Dioxide in Nitrogen & $\mathrm{SO}_{2}$ & 1500 & $\mu \mathrm{mole} / \mathrm{mole}$ \\
\hline $1664 a$ & Sulfur Dioxide in Nitrogen & $\mathrm{SO}_{2}$ & 2500 & $\mu \mathrm{mole} / \mathrm{mole}$ \\
\hline $1693 a$ & Sulfur Dioxide in Nitrogen & $\mathrm{SO}_{2}$ & 50 & $\mu \mathrm{mole} / \mathrm{mole}$ \\
\hline $1694 a$ & Sulfur Dioxide in Nitrogen & $\mathrm{SO}_{2}$ & 100 & $\mu \mathrm{mole} / \mathrm{mole}$ \\
\hline 1696 & Sulfur Dioxide in Nitrogen & $\mathrm{SO}_{2}$ & 3500 & $\mu \mathrm{mole} / \mathrm{mole}$ \\
\hline 1804 & $\begin{array}{l}\text { A.mbient Toxic Organics in } \mathrm{Ni}- \\
\text { trogen }\end{array}$ & (Fifteen co & ents, call & or details.) \\
\hline 1808 & Tetrachloroethylene in Nitro- & $\mathrm{C}_{2} \mathrm{Cl}_{4}$ & 0.25 & $\mu \mathrm{mole} / \mathrm{mole}$ \\
\hline 1809 & $\begin{array}{l}\text { Tetrachloroethylene in Nitro- } \\
\text { gen }\end{array}$ & $\mathrm{C}_{2} \mathrm{Cl}_{4}$ & 10 & $\mu \mathrm{mole} / \mathrm{mole}$ \\
\hline 2730 & Hydrogen Sulfide in Nitrogen & $\mathrm{H}_{2} \mathrm{~S}$ & 5 & $\mu \mathrm{mole} / \mathrm{mole}$ \\
\hline 2731 & Hydrogen Sulfide in Nitrogen & $\mathrm{H}_{2} \mathrm{~S}$ & 20 & $\mu \mathrm{mole} / \mathrm{mole}$ \\
\hline
\end{tabular}




\section{Permeation Devices}

These SRM's are for calibrating air pollution monitoring apparatus, and may be used to verify air pollution analytical methods and procedures. Each tube is individually certified.

SRM's 1625,1626 , and 1627 are certified over the temperature range of 20 to $30^{\circ} \mathrm{C}$. SRM $1629 \mathrm{a}$ is calibrated at $25.0^{\circ} \mathrm{C}$ only; cannot be shipped by air.

\begin{tabular}{|c|c|c|c|c|c|c|}
\hline \multirow{2}{*}{ SRM } & \multirow{2}{*}{ Type } & \multirow{2}{*}{$\begin{array}{l}\text { Tube } \\
\text { Length } \\
\text { (cm) }\end{array}$} & \multirow{2}{*}{$\begin{array}{l}\text { Permeation } \\
\text { Rate }(\mu \mathrm{g} / \\
\mathrm{min}) \text { at } 25^{\circ} \mathrm{C}\end{array}$} & \multicolumn{3}{|c|}{$\begin{array}{l}\text { Typical Concentrations (ppm) } \\
\text { Flow Rates (liters per minute) }\end{array}$} \\
\hline & & & & 1 & 5 & 10 \\
\hline 1625 & Sulfur Dioxide Permeation Tube & 10 & 2.8 & 1.07 & 0.214 & 0.107 \\
\hline 1626 & Sulfur Dioxide Permeation Tube & 5 & 1.4 & 0.535 & 0.107 & 0.0535 \\
\hline 1627 & Sulfur Dioxide Permeation Tube & 2 & 0.56 & 0.214 & 0.0428 & 0.0214 \\
\hline
\end{tabular}

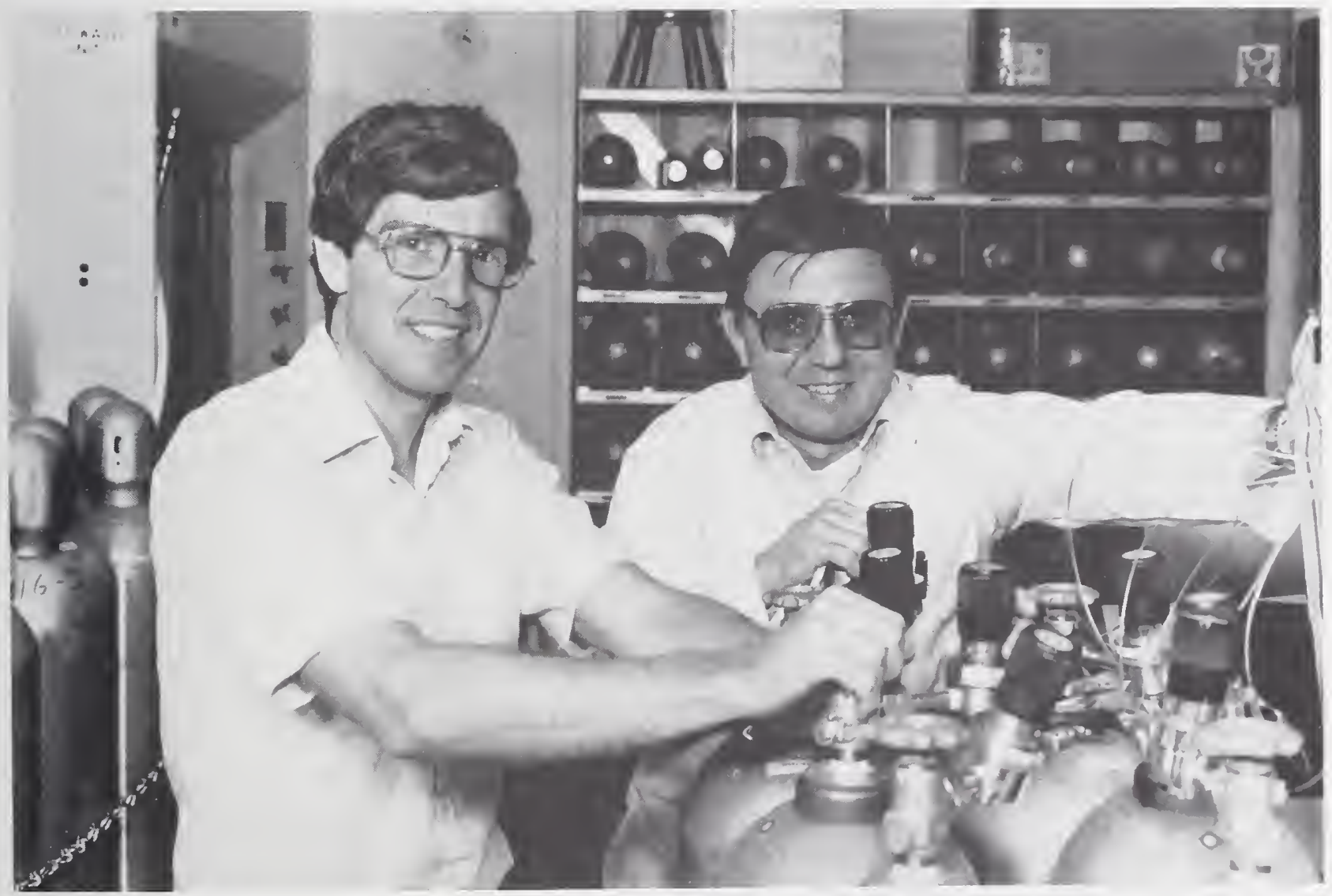

Gerald Roderick (left) and Bill Dorko, of the Gas and Particulate Science Division, connect some gas cylinders to a sampling manifold in preparation for certification measurements. 


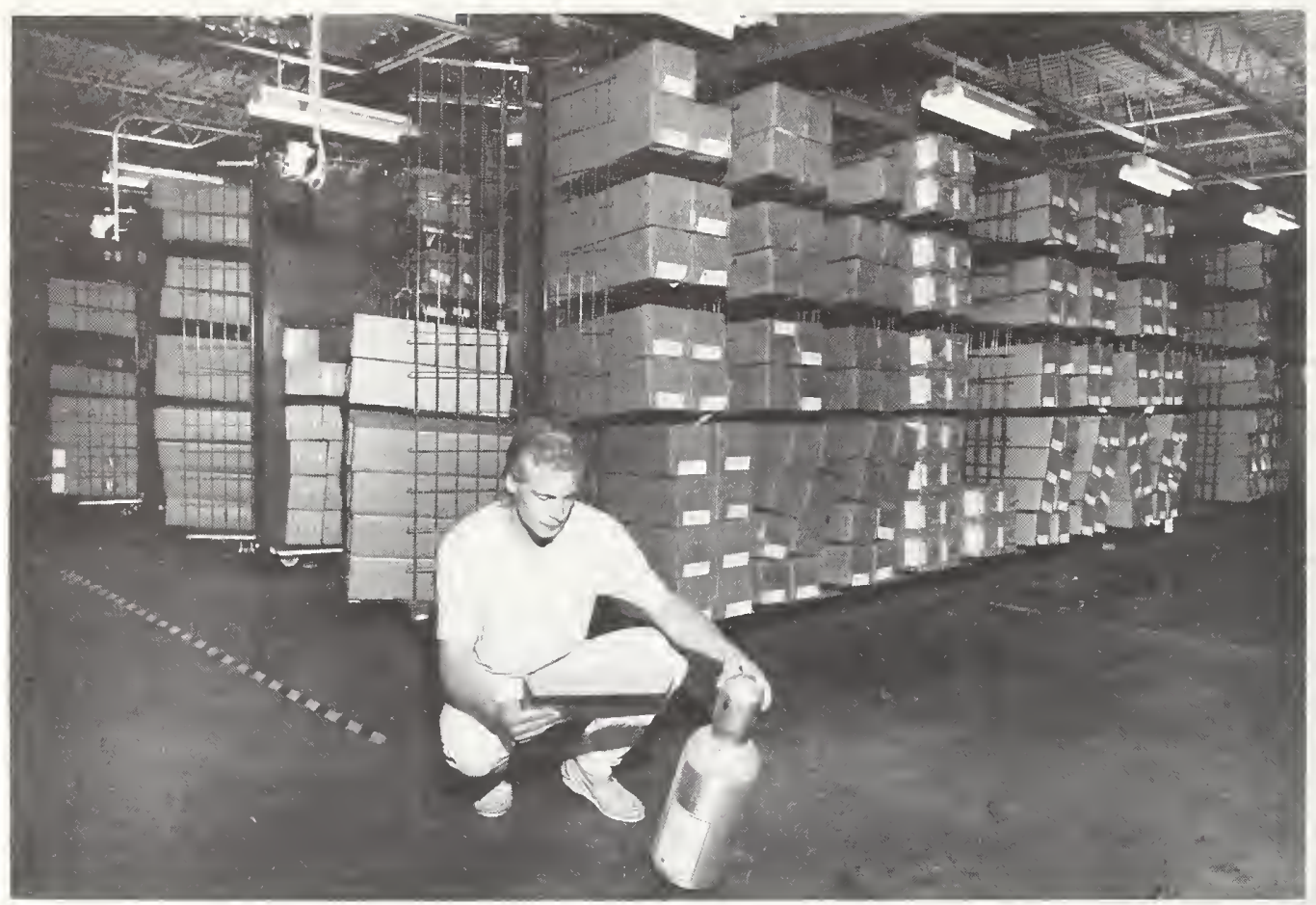

Mark Cronise checks the certificate and supplemental documentation for a gas composition SRM against the SRM and serial numbers in preparation for shipment to a customer. Mark's initiative in implementing inventory control and processing procedures have benefited the SRM program.

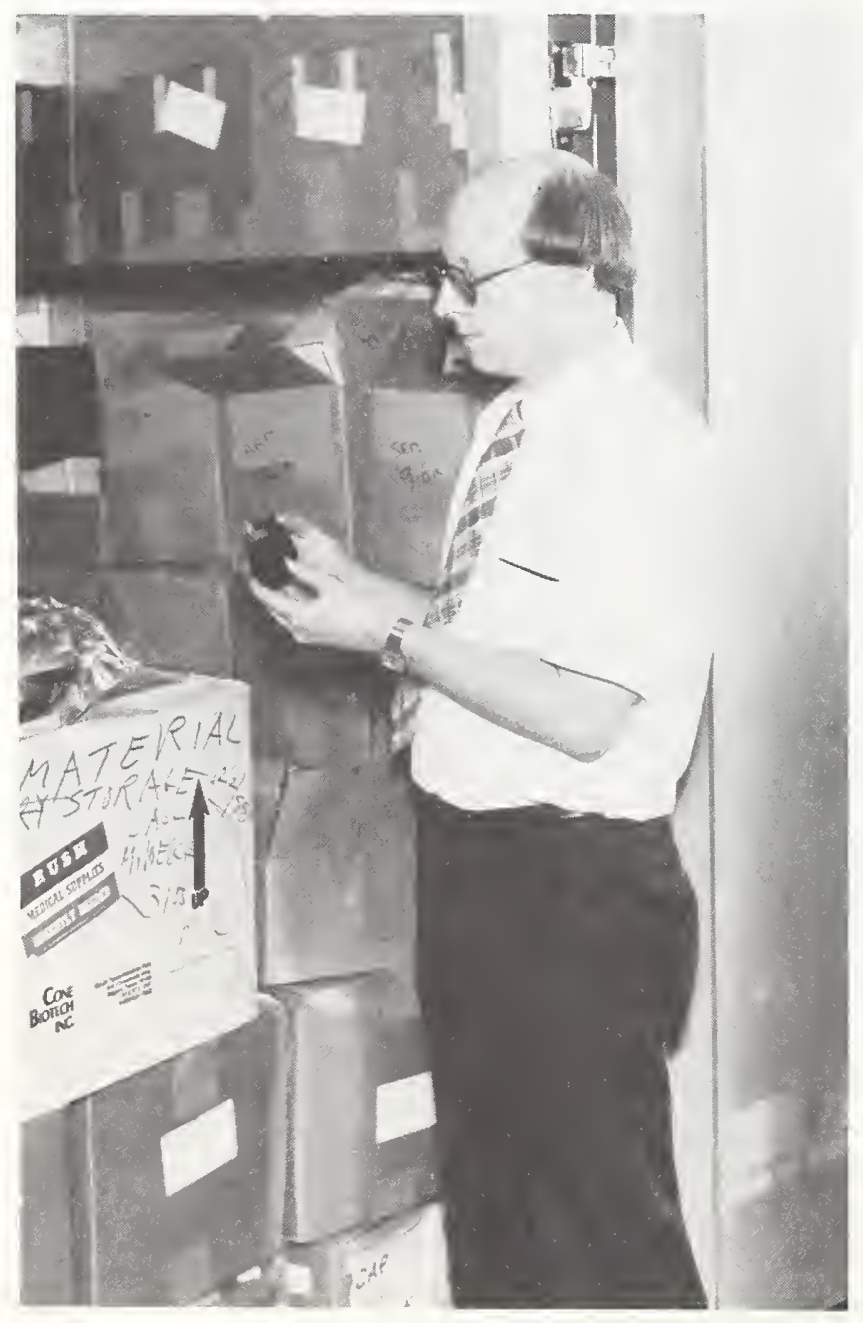

Wayne Wolf, a Research Associate with OSRM, is providing initiative and resourcefulness in the development and management of food and nutritional SRM's. Here he examines some materials that are to be distributed to the technical divisions for certification analyses. 


\section{Analyzed Liquids and Solids}

These SRM's are for analysis of materials for constituents of interest in health or environmental problems.

See also: Clinical SRM's and Industrial Hygiene SRM's.

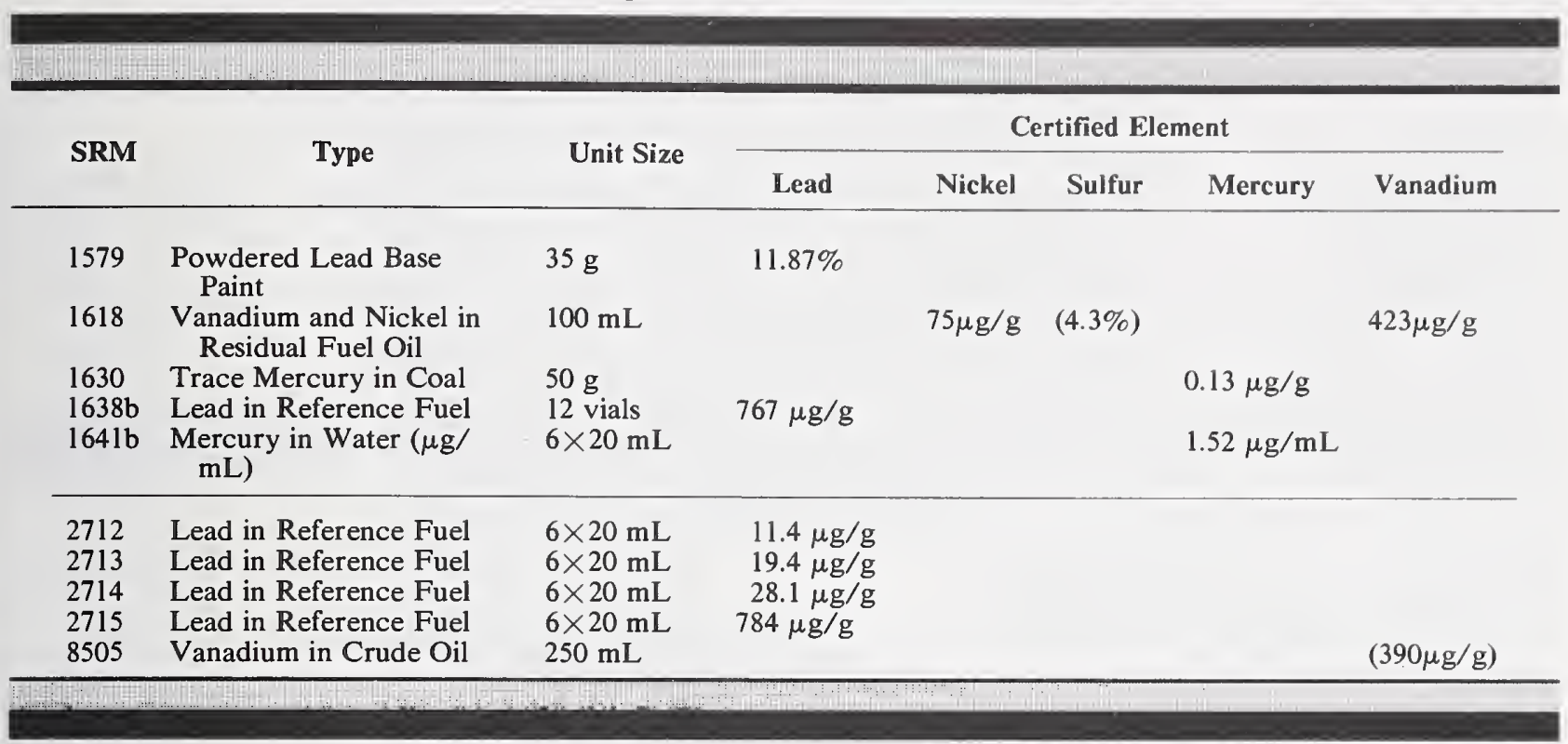

\section{Simulated Rainwaters}

These materials were developed to aid in the analysis of acidic rainwater by providing stable, homogeneous material as control standards at two levels of acidity.

NOTE: Values in parentheses are not certified.

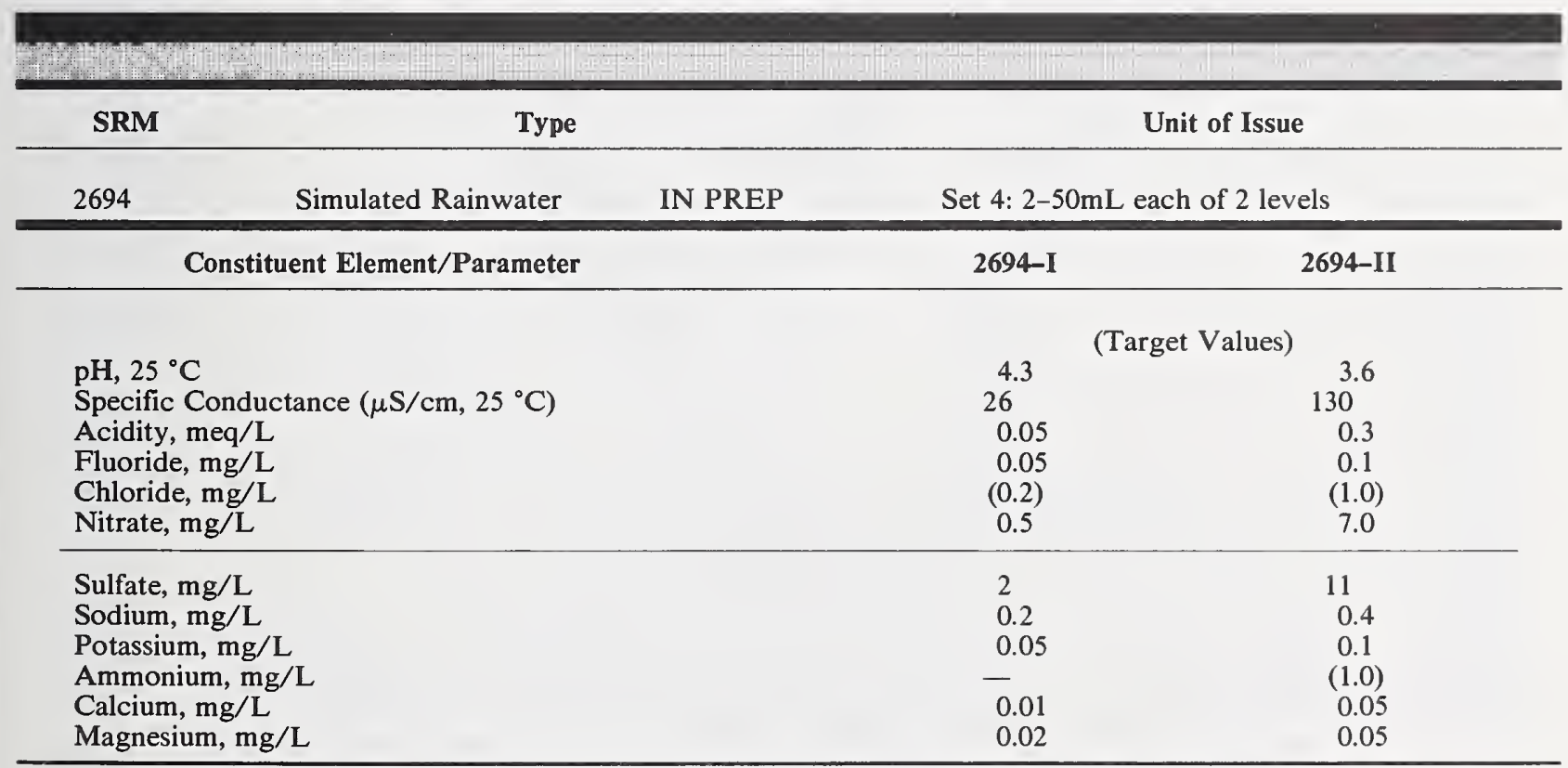

Values in parentheses are not certified, but are given for information only. 


\section{Alcohols in Reference Fuels}

These SRM's are for calibrating instruments and validating methods used to determine various alcohols in gasoline. Each SRM is issued as a set of sealed $20-\mathrm{mL}$ ampoules.

\begin{tabular}{|c|c|c|c|c|c|}
\hline \multirow[b]{2}{*}{ SRM } & \multirow[b]{2}{*}{ Type } & \multicolumn{4}{|c|}{ Nominal Concentration in Weight Percent } \\
\hline & & Wt/Unit & Methanol & Ethanol & $\begin{array}{l}\text { Methanol and } \\
\text { t-Butanol }\end{array}$ \\
\hline 1829 & Alcohols in Reference Fuel & Set (6) & 0.335 & 11.39 & $10.33+6.63$ \\
\hline 1837 & Methanol and t-Butanol & Set (5) & & & $10.33+6.63$ \\
\hline 1838 & Ethanol & Set (5) & & 11.39 & \\
\hline 1839 & Methanol & Set (5) & 0.335 & & \\
\hline
\end{tabular}

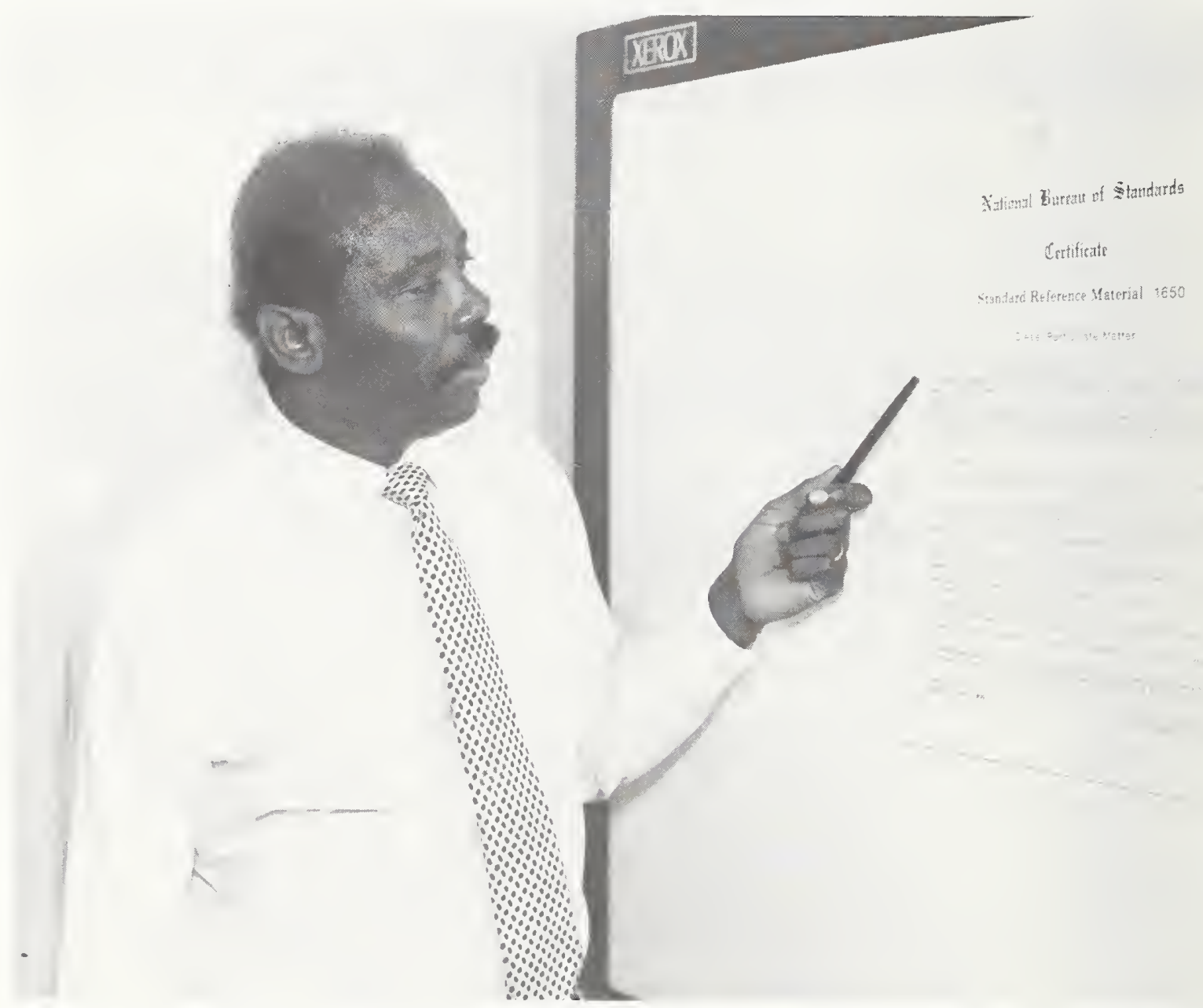

Tom Gills, Program Manager for Production and Certification, has implemented many improvements in the production and certification procedures and documentation through his initiative and determination to achieve these objectives. 


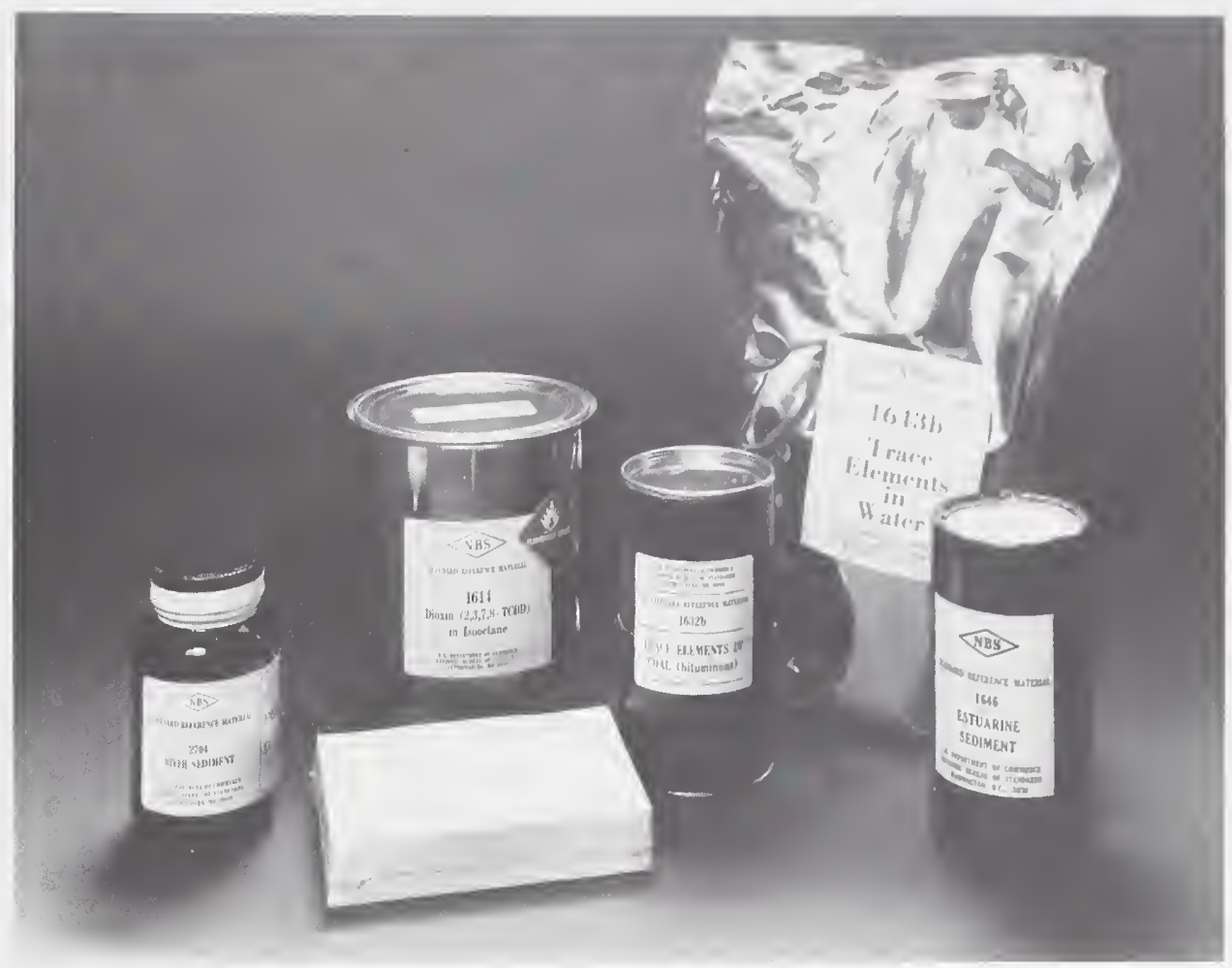

These SRM's represent the large number of environmental SRM's that are now available.

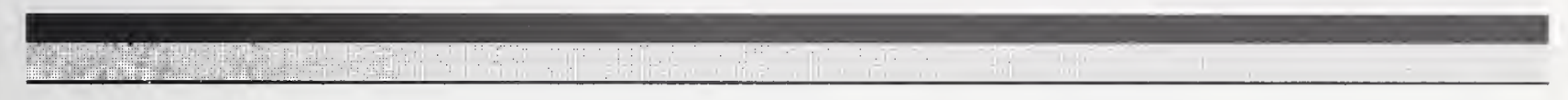

\section{Sulfur in Fossil Fuels}

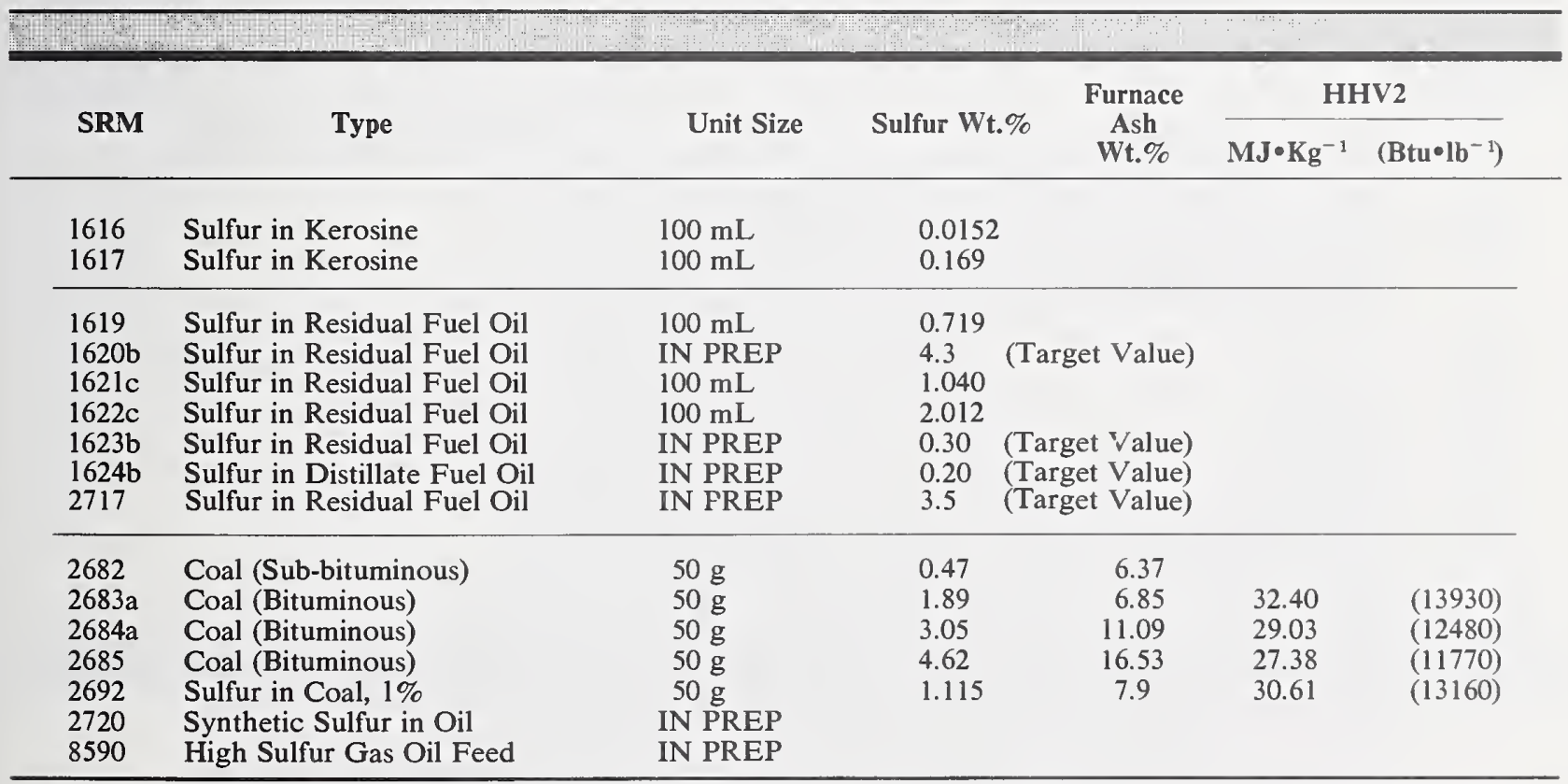

NOTE: The calorific values (MJ. $\mathrm{Kg}^{-1}$ ) may decrease upon the aging or normal oxidation of the coals. NIST will continue to monitor these calorific values and report any substantive change to the purchaser. 


\section{Trace Elements}

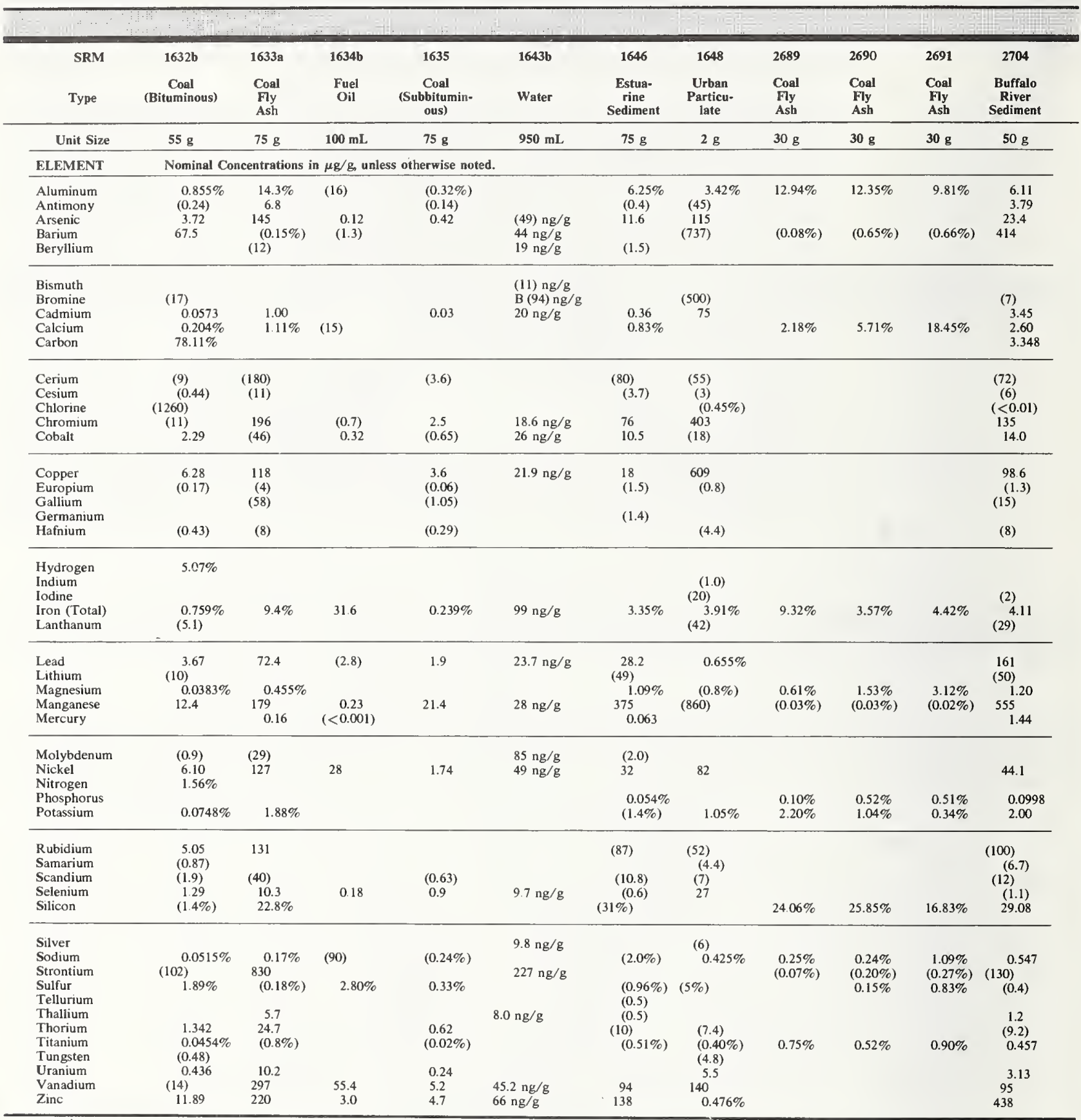

Values in parentheses are not certified, but are given for information only. 


\section{Organic Constituents}

$\begin{array}{lll}\text { SRM Type Unit of Issue } & \text { Ty }\end{array}$

\begin{tabular}{lll}
1491 & Aromatic Hydrocarbons in Hexane & 5 ampoules \\
1492 & Chlorinated Pesticides in Hexane & 5 ampoules \\
1493 & Polychlorinated Biphenyls in 2,2,4 Trimethylpentane & 5 ampoules \\
\hline
\end{tabular}

\section{$1580 \quad$ Shale Oil}

1581 Polychlorinated Biphenyls in Oil

1582 Petroleum Crude Oil

1583 Chlorinated Pesticides in Isooctane

1584 Phenols in Methanol

1585 Chlorinated Biphenyls

1586 Isotopically Labelled Priority Pollutants

1587 Nitro PAH in Methanol

1588 Organics in Cod Liver Oil

1589

1596

1597

1614

1639

Polychlorinated Biphenyls in Human Serum (as Aroclor 1260)

Dinitropyrene Isomers and 1-Nitropyrene in Methylene Chloride

Complex Mixture of Polycyclic Aromatic Hydrocarbons

Dioxin (2,3,7,8 TCDD) in Isooctane

Halocarbons (in Methanol)

1647a Priority Pollutant PAH (in Acetonitrile)

1649 Urban Dust/Organics

1650 Diesel Particulate Matter

1939 Polychlorinated Biphenyls in Sediments

1940 Polychlorinated Biphenyls in Sediments

1941 Organics in Marine Sediment

1974 Organics in Mussel Tissue

1975 Diesel Particulate Bioassay
Set of $5,2 \mathrm{~mL} /$ ampoules

Set of $8,5 \mathrm{~mL} /$ ampoules

Set of $5,2 \mathrm{~mL} /$ ampoules

Set of $6,2 \mathrm{~mL} /$ ampoules

Set of $5,2 \mathrm{~mL} /$ ampoules

Set of $5,1.2 \mathrm{~mL} /$ ampoules

Set of $6,2 \mathrm{~mL} /$ ampoules

Set of $4,1 \mathrm{~mL} /$ ampoules

Set of $5,1.2 \mathrm{~mL} /$ ampoules

Set of 3

Set of $5,1.3 \mathrm{~mL} /$ ampoules

Set of $4,1.2 \mathrm{~mL} /$ ampoules

Set of $6,1.2 \mathrm{~mL} /$ ampoules

Set of $5,1.5 \mathrm{~mL} /$ ampoules

Set of $5,1.2 \mathrm{~mL} /$ ampoules

10 grams

Set of $5,100 \mathrm{mg} /$ ampoules

IN PREP

IN PREP

IN PREP

IN PREP

IN PREP

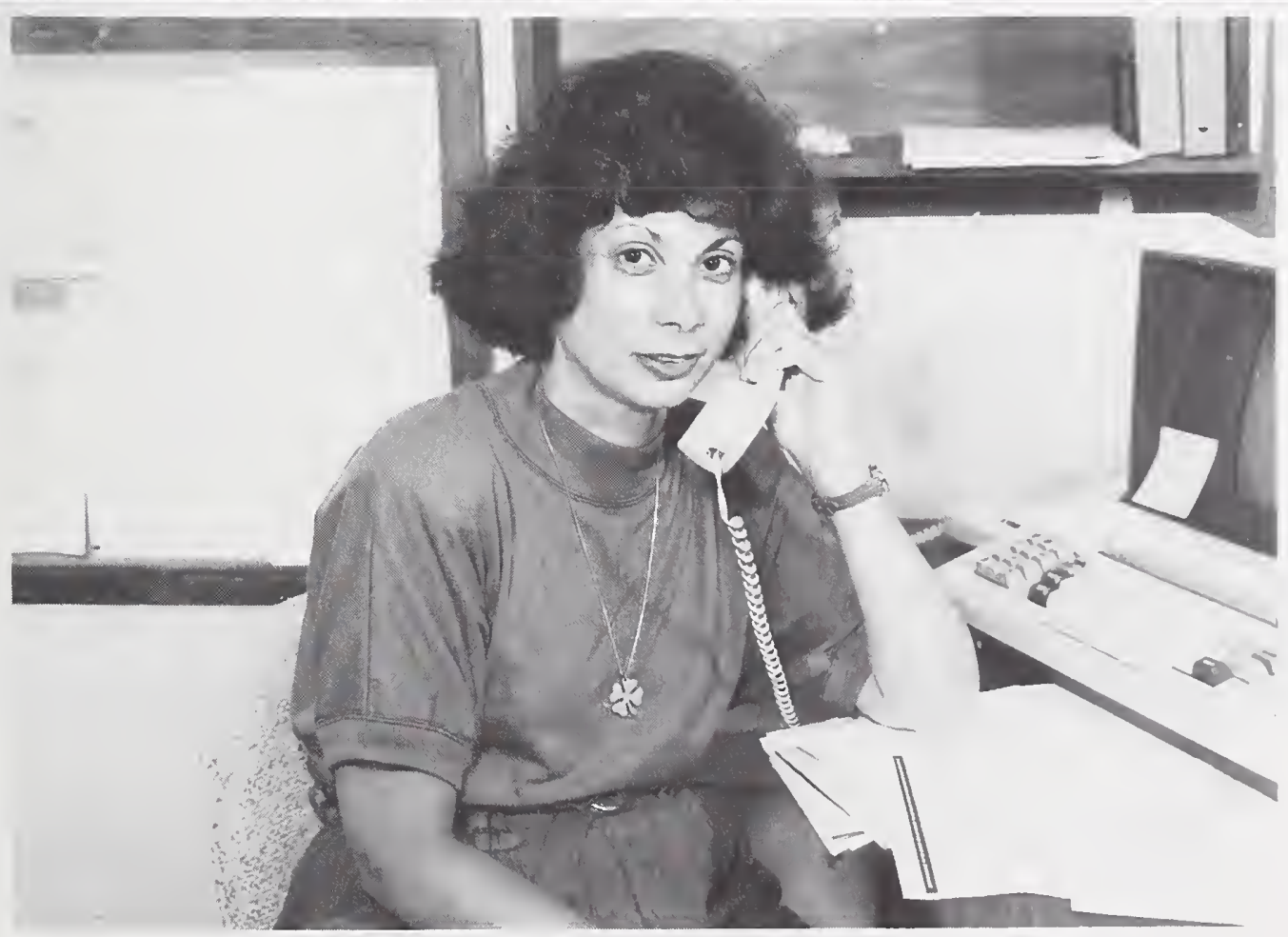

Anna Carroll, punctual and accurate, receives and processes customers orders by phone and mail. 


\section{Organic Constituents (Continued)}

\begin{tabular}{|c|c|c|c|c|c|c|c|c|}
\hline SRM & 1491 & 1580 & 1582 & & 1597 & 1647 & 1649 & 1650 \\
\hline Constituents & $(\mu \mathrm{g} / \mathrm{g})$ & $(\mu \mathrm{g} / \mathrm{g})$ & $(\mu \mathrm{g} / \mathrm{g})$ & $(\mu \mathrm{g})$ & $(\mu \mathrm{g} / \mathrm{mL})$ & $(\mu \mathrm{g} / \mathrm{mL})$ & $(\mu \mathrm{g} / \mathrm{g})$ & $(\mu \mathrm{g} / \mathrm{g})$ \\
\hline Anthracene & 11.69 & & & 101 & 87.4 & 3.29 & & \\
\hline Benz[a]anthracene & 5.37 & & 3.0 & 98.6 & 85.3 & 5.03 & 2.6 & 6.5 \\
\hline Benzo[a]pyrene & 10.14 & 21 & 1.1 & 95.8 & 82.9 & 5.30 & 2.9 & 1.2 \\
\hline Benzo[e]pyrene & 8.40 & 18 & & & & & & (10) \\
\hline Fluoranthene & 8.84 & 54 & 2.5 & & & 10.1 & 7.1 & 51 \\
\hline o-Cresol & & 385 & & & & & & \\
\hline Phenol & & 407 & & & & & & \\
\hline Perylene & 10.65 & 3.4 & 31 & 26.1 & 22.6 & & & $(0.13)$ \\
\hline Pyrene & 8.81 & 104 & & 235 & 204 & 9.84 & & 48 \\
\hline 2,6-Dimethylphenol & & 175 & & & & & & \\
\hline Benzo[f]quinoline & & 16 & & & & & & \\
\hline $\begin{array}{l}\text { (5,6-Benzoquinoline) } \\
\text { Naphthalene }\end{array}$ & 10.30 & & & 1160 & 1000 & 22.5 & & \\
\hline Acenaphthylene & 10.40 & & & & & 19.1 & & \\
\hline Acenaphthene & 10.89 & & & & & 21.0 & & \\
\hline 1-Nitropyrene & & & & & & & & 19 \\
\hline Fluorene & 10.87 & & & & & 4.92 & & \\
\hline Phenanthrene & 10.48 & & 101 & 462 & 400 & 5.06 & & (71) \\
\hline Chrysene & 10.50 & & & 71.7 & 62.0 & 4.68 & & (22) \\
\hline Benzo[b]fluoranthene & 7.85 & & & & & 5.11 & & \\
\hline Benzo[k]fluoranthene & 8.33 & & & & & 5.02 & & (2.1) \\
\hline Benzo[ghi]perylene & 7.90 & & & 53.7 & 46.5 & 4.01 & 4.5 & 2.4 \\
\hline Dibenz[a,h]anthracene & 7.74 & & & & & 3.68 & & \\
\hline Indeno[ $[1,2,3-\mathrm{cd}]$ pyrene & 9.40 & & & 602 & 52.1 & 4.06 & 3.3 & $(0.23)$ \\
\hline Dibenzothiophene & & & 33 & & & & & \\
\hline Fluoranthrene & & & & 322 & 278 & & & \\
\hline Tryphenylene & & & & 12.1 & 10.5 & & & \\
\hline 1-Methylnaphthalene & 12.4 & & & & & & & \\
\hline Biphenyl & 10.46 & & & & & & & \\
\hline 2,6-Dimethylnaphthalene & 10.8 & & & & & & & \\
\hline 2,3,5-Trimethylnaphthalene & 9.9 & & & & & & & \\
\hline 1-Methylphenanthrene & 10.4 & & & & & & & \\
\hline
\end{tabular}

Values in parentheses are not certified, but are given for information only.

\section{SRM 1639-Certified Concentration of Halocarbons at $23 \pm 3{ }^{\circ} \mathrm{C}$.}

Compound

Concentration, $\mathrm{ng} / \mu \mathrm{L}$

Chloroform

Chlorodibromomethane

Bromodichloromethane

Bromoform

124.6

389.9

Carbon Tetrachloride

86.5

Trichloroethylene

157.0

Tetrachloroethylene 
Organic Constituents (Continued)

SRM 1492 Chlorinated Pesticides in Hexane

Pesticide

Hexachlorobenzene

gamma-HCH

Heptachlor

Aldrin

Heptachlor epoxide

cis-Chlordane

trans-Nonachlor

Dieldrin

Mirex

2,4'-DDE

4, $4^{\prime}$-DDE

$2,4^{\prime}$-DDD

4,4'-DDD

$2,4^{\prime}$-DDT

4,4'-DDT

\section{Concentration $(\mathrm{ng} / \mathrm{g})$}

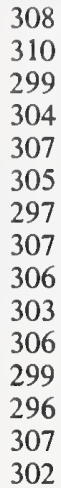

308

(304

307

305

297

307

306

303

306

299

296

SRM 1581 Polychlorinated Biphenyls in Oils

Matrix

Aroclor Type

1242

1260

1242

1260
Concentration $(\mu \mathrm{g} / \mathrm{g})$

$\begin{array}{lrr}\text { Motor Oil } & 1242 & 100 \\ \text { Motor Oil } & 1260 & 100 \\ \text { Transformer Oil } & 1242 & 100 \\ \text { Transformer Oil } & 1260 & 100\end{array}$

SRM 1583 Chlorinated Pesticides in 2,2,4-Trimethylpentane

Pesticide

Concentrations

$(\mu \mathrm{g} / \mathrm{g}) \quad\left(\mu \mathrm{g} / \mathrm{mL}, 23^{\circ} \mathrm{C}\right)$

Y-BHC (Lindane)

1.11

0.77

d-BHC

0.76

0.53

Aldrin

0.86

0.59

Heptachlor Epoxide

$(0.997)$

4,4'-DDE (p, $\left.\mathrm{p}^{\prime}-\mathrm{DDE}\right)$

1.23

0.85

$4,4^{\prime}-\mathrm{DDT}\left(\mathrm{p}, \mathrm{p}^{\prime}-\mathrm{DDT}\right)$

1.90

1.31

SRM 1584 Priority Pollutant Phenols in Methanol

\section{Compound}

Concentration $\left(\mu \mathrm{g} / \mathrm{mL}, 23^{\circ} \mathrm{C}\right)$

2-Chlorophenol

64.4

Phenol

29.7

2-Nitrophenol

25.2

2,4-Dimethylphenol

2,4-Dichlorophenol

51.6

4-Chloro-m-cresol

2,4,6-Trichlorophenol

4-Nitrophenol

4,6-Dinitro-o-cresol

Pentachlorophenol

2,4-Dinitrophenol 


\section{Organic Constituents (Continued)}

SRM 1585 Chlorinated Biphenyls in 2,2,4-Trimethylpentane (Iso octane)

Chlorinated Biphenyl

\begin{tabular}{cc} 
Concentration \\
\hline$\mu \mathrm{g} / \mathrm{g}$ & $\mu \mathrm{g} / \mathrm{mL}$ at $23.0^{\circ} \mathrm{C}$
\end{tabular}

4-Chlorobiphenyl

4,4'-Dichlorobiphenyl

43.3

29.9

$2,4,4^{\prime}$-Trichlorobiphenyl

9.53

6.57

$2,2^{\prime}, 5,5^{\prime}$-Tetrachlorobiphenyl

$3,3^{\prime}, 4,4^{\prime}$ - Tetrachlorobiphenyl

3.70

2.55

$2,2^{\prime}, 4,5,5^{\prime}$-Pentachlorobiphenyl

$2,2^{\prime}, 3,4,4^{\prime}, 5^{\prime}$-Hexachlorobiphenyl

7.72

5.32

6.62

5.24

2.37

4.56

$2,2^{\prime}, 4,4^{\prime}, 5,5^{\prime}$-Hexachlorobiphenyl

3.06

3.61

1.63

2.11

SRM 1586 Isotopically Labeled and Unlabeled Priority Pollutants in Methanol

Compound

Concentrations $(\mu \mathrm{g} / \mathrm{g})$

1586-1 (unlabeled) 1586-2 (labeled)

Carbon tetrachloride

Benzene

Chlorobenzene

Phenol

Nitrobenzene

2-Nitrophenol

2,4-Dichlorophenol

Naphthalene

Bis(2-ethylhexyl)phthalate

Benzo[a]pyrene

\begin{tabular}{rr}
128.5 & 124.4 \\
101.1 & 99.0 \\
133.0 & 144.0 \\
117.0 & 116.0 \\
126.0 & 134.5 \\
\hline 103.6 & 101.9 \\
102.5 & 82.2 \\
126.5 & 126.6 \\
63.9 & 60.4 \\
49.2 & 44.1
\end{tabular}

SRM 1587 Nitrated Polycyclic Aromatic Hydrocarbons in Methanol

Compound

\section{Concentrations}

$(\mu \mathrm{g} / \mathrm{g}) \quad\left(\mu \mathrm{g} / \mathrm{mL}, 23^{\circ} \mathrm{C}\right)$

2-Nitrofluorene

9-Nitroanthracene

3-Nitrofluoranthene

9.24

7.30

1-Nitropyrene

7-Nitrobenz[a]anthracene

8.95

9.27

7.07

6-Nitrochrysene

8.13

7.32

6-Nitrobenzo[a]pyrene

(6.1)

(4.8) 


\section{Organic Constituents (Continued)}

SRM 1588 Organics in Cod Liver Oil

Compound

Concentration (ng/g)

Hexachlorobenzene

alpha- $\mathrm{HCH}$

148

trans-Chlordane

86

cis-Chlordane

50

trans-Nonachlor

158

Dieldrin

209

4,4'-DDD

150

277

4,4'-DDE

641

2,4'-DDT

156

4,4'-DDT

PCB 101

PCB 138

PCB 170

PCB 180

SRM 1589 PCB's in Human Serum

Compound

Concentrations

(ng/g)

(ng/mL)

Aroclor 1260

106.0

107.9

SRM 1596 Dinitropyrene Isomers and 1-Nitropyrene in Methylene Chloride

Compound

Concentration

$\mu \mathrm{g} / \mathrm{g} \quad \mu \mathrm{g} / \mathrm{mL}$

1-Nitropyrene

4.38

2.10

4.82

5.81

1,3-Dinitropyrene

6.39

1,8-Dinitropyrene

7.90

10.48

SRM 1614 Dioxin (2,3,7,8-TCDD in Isooctane)

Compound

Concentrations

(ng/g) $\quad\left(\mathrm{ng} / \mathrm{mL}, 23^{\circ} \mathrm{C}\right)$

2,3,7,8-TCDD

98.3

67.8

2,3,7,8-TCDD- ${ }^{13} \mathrm{C}$

95.6

65.9

\section{GC/MS System Performance}

These SRM's are for evaluating the sensitivity of gas chromatographic/mass spectrometry (GC/MS) instrumentation. They consist of two concentrations each of methyl stearate and benzophenone.

\begin{tabular}{ccccc}
\hline \multirow{2}{*}{ SRM } & Type & \multicolumn{2}{c}{ Concentrations $(\mathrm{ng} / \mu \mathrm{L})$} & \multicolumn{2}{c}{ Unit Size } \\
\cline { 3 - 5 } & & $\begin{array}{c}\text { Methyl } \\
\text { Stearate }\end{array}$ & Benzophenone \\
\hline & & & & \\
1543 & GC/MS System Performance & $0.99 ; 4.98$ & $1.01 ; 5.01$ & 1 Set, 4 vials \\
\hline
\end{tabular}




\section{Industrial Hygiene}

These SRM's were developed for industrial hygiene analyses to provide reference materials for toxicology research and for monitoring human exposure to selected toxic elements.

\section{Freeze-Dried Urine}

SRM's 2670, 2671a, and 2672a consist of freeze-dried urine in $30 \mathrm{~mL}$ serum bottles. The freeze-dried urine SRM's are to be reconstituted by the addition of $20 \mathrm{~mL}$ of pure water to each bottle. Each unit contains a set of four bottles, two bottles each at normal and elevated levels.

\begin{tabular}{|c|c|c|c|c|c|c|c|c|}
\hline SRM & \multicolumn{4}{|c|}{2670} & \multicolumn{2}{|c|}{ 2671a } & \multicolumn{2}{|c|}{$2672 \mathrm{a}$} \\
\hline \multirow[t]{2}{*}{ Type } & \multicolumn{4}{|c|}{ Toxic Metals } & \multicolumn{2}{|c|}{ Fluoride } & \multicolumn{2}{|c|}{ Mercury } \\
\hline & $\begin{array}{l}\text { Lor } \\
\text { Leve }\end{array}$ & $\begin{array}{l}\text { w } \\
\text { vel }\end{array}$ & & $\begin{array}{l}\text { evated } \\
\text { Level }\end{array}$ & $\begin{array}{l}\text { Low } \\
\text { Level }\end{array}$ & $\begin{array}{c}\text { Elevated } \\
\text { Level }\end{array}$ & $\begin{array}{l}\text { Low } \\
\text { Level }\end{array}$ & $\begin{array}{c}\text { Elevated } \\
\text { Level }\end{array}$ \\
\hline $\begin{array}{l}\text { Aluminum } \\
\text { Arsenic } \\
\text { Beryllium } \\
\text { Cadmium } \\
\text { Calcium }\end{array}$ & $\begin{array}{c}(0.18) \\
(0.06) \\
(\leqslant 0.0005) \\
(0.00040) \\
0.105 \\
\end{array}$ & $\begin{array}{l}\mu \mathrm{g} / \mathrm{mL} \\
\mu \mathrm{g} / \mathrm{mL} \\
\mu \mathrm{g} / \mathrm{mL} \\
\mu \mathrm{g} / \mathrm{mL} \\
\mathrm{mg} / \mathrm{mL}\end{array}$ & $\begin{array}{c}(0.18) \\
0.48 \\
(0.033) \\
0.088 \\
0.105\end{array}$ & $\begin{array}{l}\mu \mathrm{g} / \mathrm{mL} \\
\mu \mathrm{g} / \mathrm{mL} \\
\mu \mathrm{g} / \mathrm{mL} \\
\mu \mathrm{g} / \mathrm{mL} \\
\mathrm{mg} / \mathrm{mL}\end{array}$ & & & & \\
\hline $\begin{array}{l}\text { Chloride } \\
\text { Chromium } \\
\text { Copper } \\
\text { Gold } \\
\text { Lead }\end{array}$ & $\begin{array}{l}(4.4) \\
(0.013) \\
0.13 \\
(0.008) \\
(0.01)\end{array}$ & $\begin{array}{l}\mathrm{mg} / \mathrm{mL} \\
\mu \mathrm{g} / \mathrm{mL} \\
\mu \mathrm{g} / \mathrm{mL} \\
\mathrm{ng} / \mathrm{mL} \\
\mu \mathrm{g} / \mathrm{mL}\end{array}$ & $\begin{array}{l}(4.4) \\
0.085 \\
0.37 \\
(0.24) \\
0.109\end{array}$ & $\begin{array}{l}\mathrm{mg} / \mathrm{mL} \\
\mu \mathrm{g} / \mathrm{mL} \\
\mu \mathrm{g} / \mathrm{mL} \\
\mu \mathrm{g} / \mathrm{mL} \\
\mu \mathrm{g} / \mathrm{mL}\end{array}$ & $0.55 \mathrm{mg} / \mathrm{L}$ & $5.7 \mathrm{mg} / \mathrm{L}$ & & \\
\hline $\begin{array}{l}\text { Magnesium } \\
\text { Manganese } \\
\text { Mercury } \\
\text { Nickel } \\
\text { Platinum }\end{array}$ & $\begin{array}{l}0.063 \\
(0.03) \\
(0.002) \\
(0.07) \\
(0.008)\end{array}$ & $\begin{array}{l}\mathrm{mg} / \mathrm{mL} \\
\mu \mathrm{g} / \mathrm{mL} \\
\mu \mathrm{g} / \mathrm{mL} \\
\mu \mathrm{g} / \mathrm{mL} \\
\mathrm{ng} / \mathrm{mL}\end{array}$ & $\begin{array}{c}0.063 \\
(0.33) \\
0.105 \\
(0.30) \\
(0.12)\end{array}$ & $\begin{array}{l}\mathrm{mg} / \mathrm{mL} \\
\mu \mathrm{g} / \mathrm{mL} \\
\mu \mathrm{g} / \mathrm{mL} \\
\mu \mathrm{g} / \mathrm{mL} \\
\mu \mathrm{g} / \mathrm{mL}\end{array}$ & & & $(0.002) \mathrm{mg} / \mathrm{L}$ & $0.105 \mathrm{mg} / \mathrm{L}$ \\
\hline $\begin{array}{l}\text { Potassium } \\
\text { Selenium } \\
\text { Sodium } \\
\text { Sulfate } \\
\text { Vanadium }\end{array}$ & $\begin{array}{l}(1.5) \\
0.030 \\
2.62 \\
(1.3)\end{array}$ & $\begin{array}{l}\mathrm{mg} / \mathrm{mL} \\
\mu \mathrm{g} / \mathrm{mL} \\
\mathrm{mg} / \mathrm{mL} \\
\mathrm{mg} / \mathrm{mL}\end{array}$ & $\begin{array}{c}(1.5) \\
0.46 \\
2.62 \\
(1.3) \\
(0.12)\end{array}$ & $\begin{array}{c}\mathrm{mg} / \mathrm{mL} \\
\mu \mathrm{g} / \mathrm{mL} \\
\mathrm{mg} / \mathrm{mL} \\
\mathrm{mg} / \mathrm{mL} \\
\mu \mathrm{g} / \mathrm{mL}\end{array}$ & & & & \\
\hline
\end{tabular}

Values in parentheses are not certified, but are given for information only.

\section{Freeze-Dried Urine (Continued)}

\begin{tabular}{|c|c|c|c|}
\hline SRM & 1507 & 1508 & RM 8444 \\
\hline Type & THC in Urine & Cocaine in Urine & Cotinine in Urine \\
\hline Unit Size & 3 bottles & IN PREP & 4 vials \\
\hline Certified Concentration & $20 \mathrm{ng} / \mathrm{mL}$ & & $\begin{array}{c}\text { Blank level } 0.8 \mathrm{ng} / \mathrm{g} \\
\text { Low level } 54 \mathrm{ng} / \mathrm{g} \\
\text { High level } 488 \mathrm{ng} / \mathrm{g}\end{array}$ \\
\hline
\end{tabular}




\section{Thin Films for X-ray Fluorescence}

These SRM's are for standardizing x-ray spectrometers. They may be useful in elemental analysis of particulate matter collected on filter media, and where x-ray spectrometer calibration functions are determined using thin film standards. Each SRM is individually certified and consists of a silica-base glass film $(0.5 \mu \mathrm{m}$ thick) deposited on a $47 \mathrm{~mm}$ diameter polycarbonate filter, mounted on an aluminum ring.

\begin{tabular}{|c|c|c|c|c|c|c|c|c|c|c|c|c|c|}
\hline \multirow{2}{*}{ SRM } & \multirow{2}{*}{ Type } & \multicolumn{12}{|c|}{ Chemical Composition (Nominal $\mu \mathrm{g} / \mathrm{cm}^{2}$ ) } \\
\hline & & Al & $\mathrm{Ca}$ & Co & $\mathrm{Cu}$ & $\mathrm{Fe}$ & $\mathrm{Pb}$ & $\mathbf{K}$ & $\mathrm{Mn}$ & $\mathrm{Si}$ & $\mathrm{Ti}$ & V & $\mathrm{Zn}$ \\
\hline $\begin{array}{l}1832 \\
1833\end{array}$ & $\begin{array}{l}\text { Thin-Glass Film } \\
\text { Thin-Glass Film }\end{array}$ & 15 & 20 & 1 & 2 & 15 & 17 & 18 & 5 & $\begin{array}{l}36 \\
35\end{array}$ & 14 & 5 & 4 \\
\hline
\end{tabular}

\section{Materials on Filter Media}

These SRM's consist of potentially hazardous materials deposited on filters to be used to determine the levels of these materials in industrial atmospheres.

\begin{tabular}{|c|c|c|c|c|c|c|c|}
\hline \multirow{2}{*}{ SRM } & \multirow{2}{*}{ Type } & \multirow{2}{*}{ Unit Size } & \multirow{2}{*}{$\begin{array}{l}\text { Material } \\
\text { Certified }\end{array}$} & \multicolumn{4}{|c|}{ Quantity Certified ( $\mu \mathrm{g} /$ filter) } \\
\hline & & & & I & II & III & IV \\
\hline $2676 c$ & Metals on Filter Media & Set of 8 & $\begin{array}{l}\text { Cadmium } \\
\text { Lead } \\
\text { Manganese } \\
\text { Zinc }\end{array}$ & $\begin{array}{l}0.954 \\
7.47 \\
2.11 \\
9.99\end{array}$ & $\begin{array}{r}2.83 \\
14.92 \\
9.92 \\
49.68\end{array}$ & $\begin{array}{l}10.09 \\
29.81 \\
19.85 \\
99.28\end{array}$ & $\begin{array}{l}(<0.01) \\
(<0.01) \\
(<0.01) \\
(<0.01)\end{array}$ \\
\hline 2677 & $\begin{array}{l}\text { Beryllium and Arsenic } \\
\text { on Filter Media }\end{array}$ & 2 sets of 4 & $\begin{array}{l}\text { Beryllium } \\
\text { Arsenic }\end{array}$ & $\begin{array}{l}0.052 \\
0.103\end{array}$ & $\begin{array}{l}0.256 \\
1.07\end{array}$ & $\begin{array}{l}1.03 \\
10.5\end{array}$ & $\begin{array}{l}<0.001 \\
<0.002\end{array}$ \\
\hline $2679 a$ & Quartz on Filter Media & Set of 4 & $\begin{array}{l}\text { Quartz } \\
\text { Clay }\end{array}$ & $\begin{array}{c}<2 \\
(370)\end{array}$ & $\begin{array}{l}30.8 \\
(370)\end{array}$ & $\begin{array}{l}80.2 \\
(370)\end{array}$ & $\begin{array}{l}202.7 \\
(370)\end{array}$ \\
\hline
\end{tabular}

Values in parentheses are not certified, but are given for information only.

\section{Blank Filters}

“These SRM's, except for SRM's 2678-81, consist of potentially hazardous materials deposited on filter media to be used to determine the levels of these materials in industrial atmospheres. SRM's 2678-81 are blank filters that have been analyzed at NIST and are to be used in the assessment of system blank and in the evaluation of detection limits."

\begin{tabular}{ccccc}
\hline SRM & Type & Diameter & Pore Size & Nominal \\
\hline 2678 & $\begin{array}{l}\text { Cellulose Acetate } \\
\text { Membrane } \\
\text { Cellulose Acetate } \\
\text { Membrane } \\
\text { Ashless }\end{array}$ & $47 \mathrm{~mm}$ & 0.45 micron & 0.09 \\
2680 & $37 \mathrm{~mm}$ & 0.80 micron & 0.05 \\
\hline
\end{tabular}



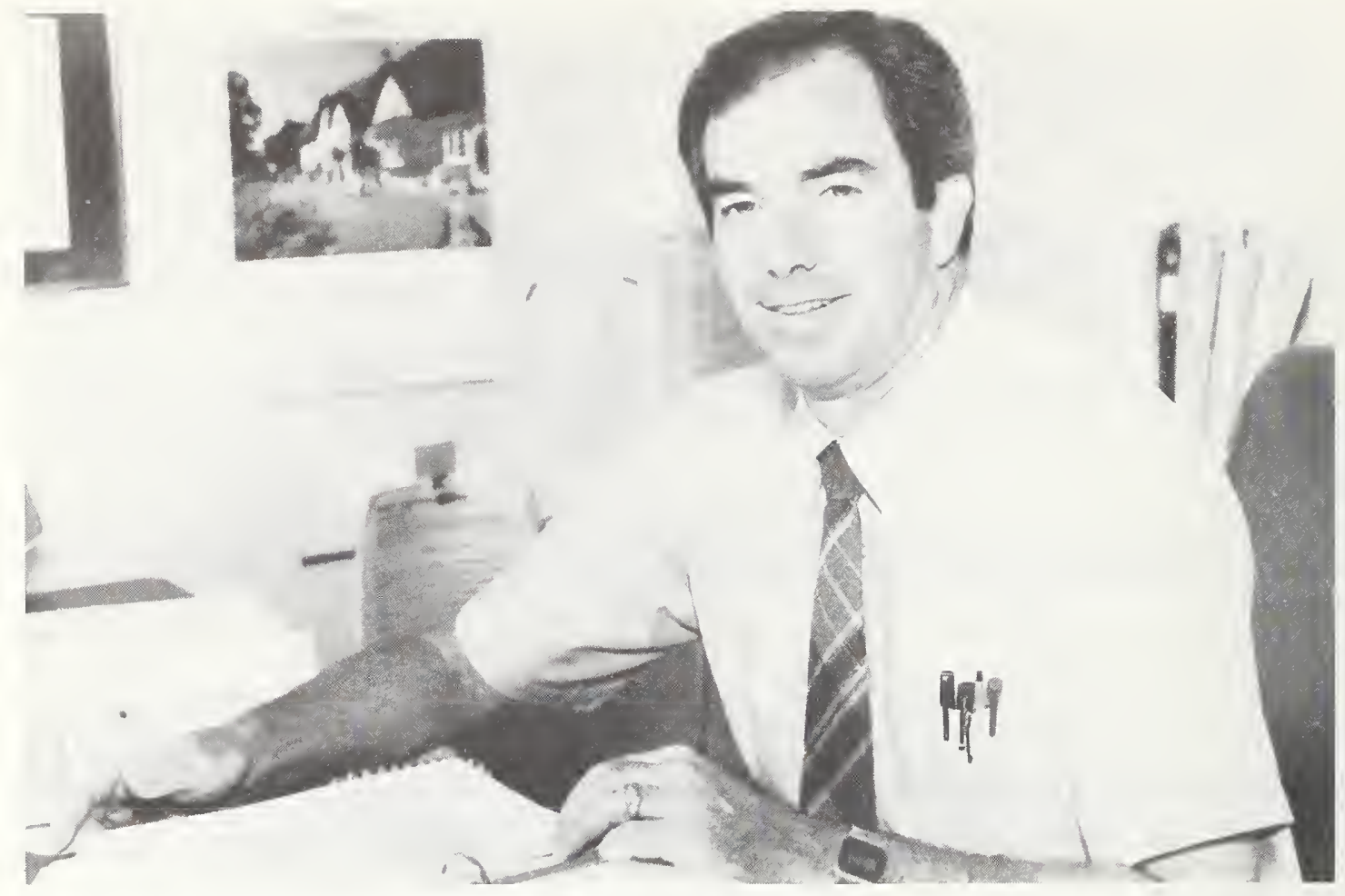

Ray McKenzie, a project manager for OSRM, provides responsive, creative leadership for a variety of physical properties and engineering SRM's.

\section{Respirable Quartz}

These SRM's consists of quartz powders that are in the respirable size range. They are intended for use in determining the level of quartz in an industrial atmosphere by $x$-ray diffraction.

\begin{tabular}{cccc}
\hline SRM & Type & Constituent Certified & Amount \\
\hline \multirow{2}{*}{1878} & Alpha Quartz & $95.5 \%$ Crystalline $\alpha$-quartz & $5 \mathrm{~g}$ \\
1879 & Cristobalite & $98.0 \%$ Crystalline Cristobalite & $5 \mathrm{~g}$ \\
\hline
\end{tabular}




\section{Asbestos}

These SRM's consist of four $3 \times 3 \mathrm{~mm}$ sections of a $0.4 \mathrm{~mm}$ pore size polycarbonate filter containing chrysotile fibers mixed with an urban dust or with grunerite fibers. They are intended for use in evaluating the techniques used to count and identify chrysotile and grunerite asbestos fibers in filter samples by transmission electron microscopy.

\begin{tabular}{|c|c|c|}
\hline SRM & Type & Fiber Loading \\
\hline $\begin{array}{l}1876 a \\
8410 \\
8411\end{array}$ & $\begin{array}{l}\text { Chrysotile Asbestos } \\
\text { Chrysotile Asbestos Research Filter } \\
\text { Mixed asbestos } \\
\text { Research Filter }\end{array}$ & $\begin{array}{l}37 \text { fibers } / 0.01 \mathrm{~mm}^{2} \\
7.9 \text { fibers } / 0.01 \mathrm{~mm}^{2} \\
138 \text { fibers } / 0.01 \mathrm{~mm} \text { Chrysotile } \\
43 \text { fibers } / 0.01 \mathrm{~mm} \text { Grunerite }\end{array}$ \\
\hline
\end{tabular}

SRM 1866, Bulk Asbestos-Common, is a set of the three common bulk mine-grade asbestos materials and one synthetic glass fiber sample. There are 4-5 grams of each sample in the set of materials. The three asbestos types are Chrysotile, Grunerite (Amosite), and Riebeckite (Crocidolite). The optical properties of each of these materials observed by polarized light microscopy have been characterized so that these samples may serve as primary calibration standards for the identification of asbestos types in building material. SRM 1867, Bulk Assbestos-Uncommon, will contain three materials: Anthophylite, Actinolite, and Tremolite and will be used in the same manner and for the same purpose as SRM 1866.

\begin{tabular}{lll} 
& & \\
\hline SRM & Type & \\
\hline
\end{tabular}

1866 Bulk Asbestos-Common

1867 Bulk Asbestos-Uncommon (IN PREP)

Chrysotile, Grunerite, Riebeckite Anthophylite, Actinolite, Tremolite

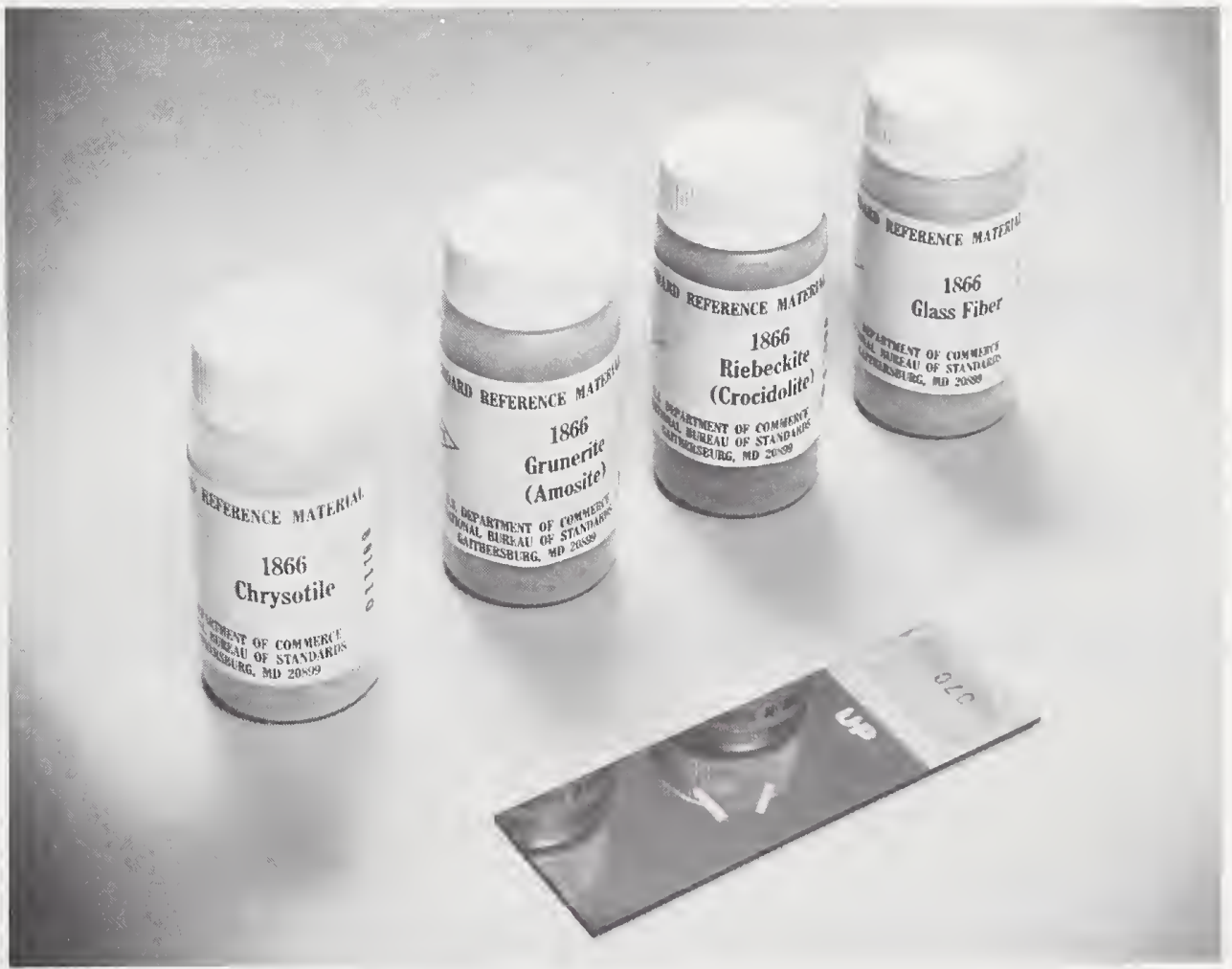

SRM 1866, Bulk Asbestos (a set of four materials), our newest asbestos SRM, joins our other available asbestos SRM's - Chrysotile Filter (SRM 1876a) and Research Filters (8410 and 8411). 


\section{Lubricating Materials}

\section{Metallo-Organic Compounds}

These SRM's are for preparing solutions in oils of known and reproducible concentrations of metals. Certificates give directions for preparing a solution of known concentration in lubricating oil.

\begin{tabular}{|c|c|c|c|c|}
\hline \multirow[b]{2}{*}{ SRM } & \multirow[b]{2}{*}{ Туре } & \multicolumn{3}{|c|}{ Constituent Certified } \\
\hline & & Element & $\begin{array}{c}\text { (Wt. } \\
\text { percent) }\end{array}$ & $\begin{array}{l}\text { Wt/Unit } \\
\text { (grams) }\end{array}$ \\
\hline $1075 \mathrm{a}$ & Aluminum 2-ethylhexanoate & Aluminum & 8.07 & 5 \\
\hline $1051 b$ & Barium cyclohexanebutyrate & Barium & 28.7 & 5 \\
\hline $1053 a$ & Cadmium cyclohexanebutyrate & Cadmium & 24.8 & 5 \\
\hline $1078 b$ & Tris (1-phenyl-1,3-butanediono)chromium (III) & Chromium & 9.6 & 5 \\
\hline $1080 \mathrm{a}$ & Bis(1-phenyl-1,3-butanediono)copper (II) & Copper & 16.37 & 5 \\
\hline $1079 b$ & Tris (1-phenyl-1,3-butanediono)iron (III) & Iron & 10.45 & 5 \\
\hline $1059 c$ & Lead cyclohexanebutyrate & Lead & 37.5 & 5 \\
\hline $1060 \mathrm{a}$ & Lithium cyclohexanebutyrate & Lithium & 4.1 & 5 \\
\hline $1061 \mathrm{c}$ & Magnesium cyclohexanebutyrate & Magnesium & 6.45 & 5 \\
\hline $1065 b$ & Nickel cyclohexanebutyrate & Nickel & 13.89 & 5 \\
\hline $1071 \mathrm{~b}$ & Triphenyl phosphate & Phosphorus & 9.48 & 5 \\
\hline $1066 \mathrm{a}$ & Octaphenylcyclotetrasiloxane & Silicon & 14.14 & 5 \\
\hline $1077 \mathrm{a}$ & Silver 2-ethylhexanoate & Silver & 42.60 & 5 \\
\hline $1069 b$ & Sodium cyclohexanebutyrate & Sodium & 12.0 & 5 \\
\hline $1070 \mathrm{a}$ & Strontium cyclohexanebutyrate & Strontium & 20.7 & 5 \\
\hline $1057 \mathrm{~b}$ & Dibutyltin bis (2-ethylhexanoate) & Tin & 22.95 & 5 \\
\hline $1052 b$ & Bis(1-phenyl-1,3-butanediono)oxovanadium (IV) & Vanadium & 13.01 & 5 \\
\hline $1073 b$ & Zinc cyclohexanebutyrate & Zinc & 16.66 & 5 \\
\hline
\end{tabular}

\section{Lubricating Base Oils}

Each of these SRM's consists of a series of five concentrations (5 bottles, $20 \mathrm{~g}$ each) of a single element in a base oil.

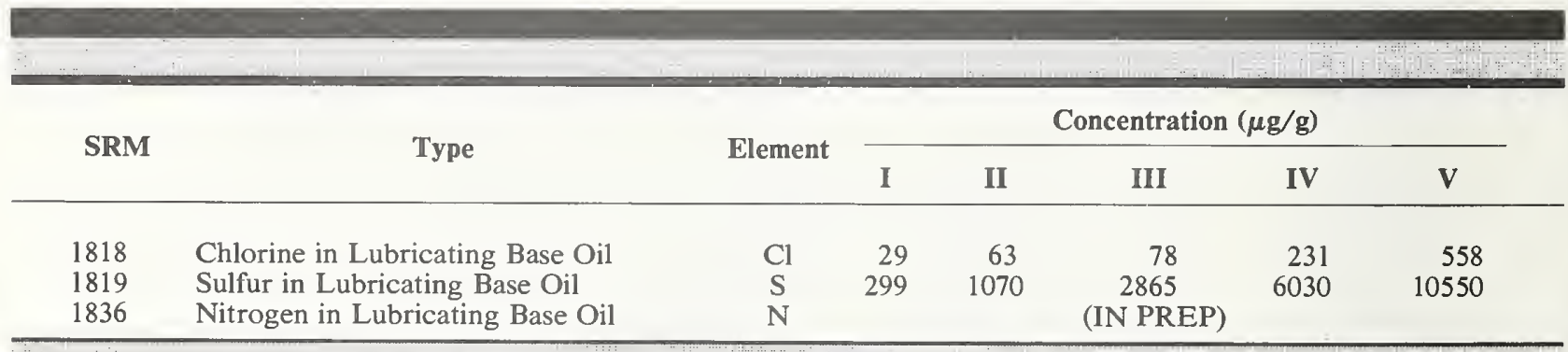




\section{Catalyst Package for Lubricant Oxidation}

SRM $1817 \mathrm{~b}$ is intended primarily for use in evaluating the oxidation stability of lubricating oils, i.e., automative crankcase lubricants. The SRM contains: (1) an oxidized/nitrated fuel fraction, (2) a metal naphthenate mixture, and (3) distilled water. The metal naphthenate mixture has the following weight percentages of metal naphthenates: lead-82, iron-7, copper-4, manganese-3.5, and tin-3.5. SRM 1817b is available as a kit of 5 ampoules of each of the three components. The fuel and metal catalysts are sealed under inert atmosphere to ensure their stabilities.

\begin{tabular}{|l|l|l|l|}
\hline \\
\hline
\end{tabular}

\section{Wear-Metals in Oil}

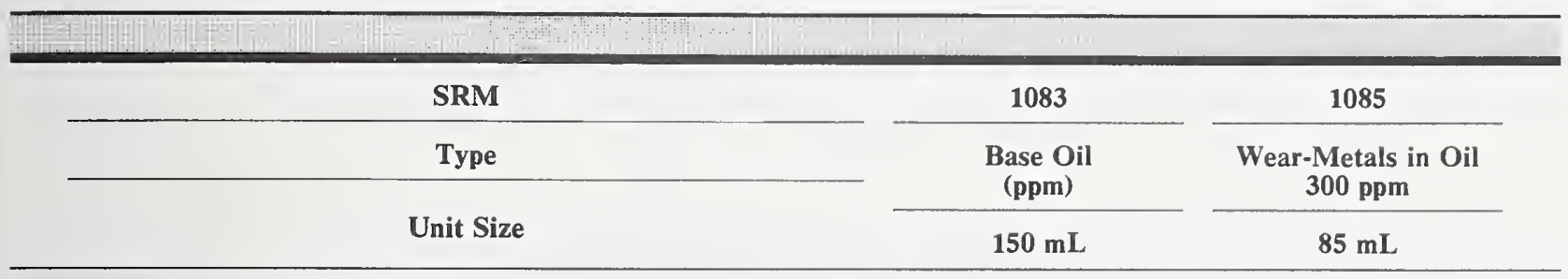

ELEMENT (Values in $\mu \mathrm{g} / \mathrm{g}$ )

\begin{tabular}{llc} 
Aluminum & $(<0.5)$ & 296 \\
Chromium & $(<0.02)$ & 298 \\
Copper & $(<0.5)$ & 295 \\
Iron & $(<1)$ & 300 \\
\hline Lead & $(<0.04)$ & $(305)$ \\
Magnesium & $(<0.1)$ & 297 \\
Molybdenum & $(<0.01)$ & 292 \\
Nickel & $(<0.4)$ & 303 \\
Silicon & $(<1)$ & $(308)$ \\
\hline Silver & $(<0.05)$ & $(291)$ \\
Sulfur & $(980)$ & $(4806)$ \\
Tin & $(<0.4)$ & 296
\end{tabular}

Values in parentheses are not certified, but are given for information only.

Varm

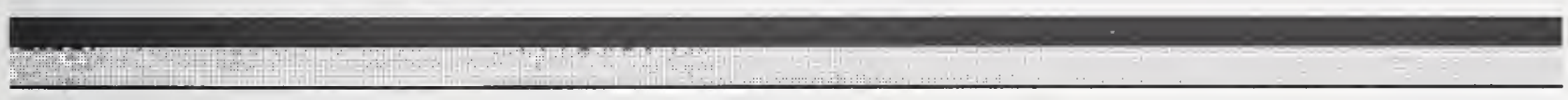

\section{Catalyst Characterization Materials}

\begin{tabular}{lll}
\hline SRM & \multicolumn{1}{c}{ Type } & Wt/Unit (grams) \\
\hline & & $25 \mathrm{gm}$ \\
RM8580 & Nickel on Fresh Alumina (NiO 2.92 Wt \%) & $25 \mathrm{gm}$ \\
RM8581 & Nickel on Fresh Alumina (NiO 14.35 Wt \%) & $25 \mathrm{gm}$ \\
RM8582 & Nickel on Fresh Alumina (NiO 54.97 Wt \%) & $50 \mathrm{gm}$ \\
RM8583 & Platinum on Fresh Alumina (Pt 0.5760 Wt \%) & $50 \mathrm{gm}$ \\
RM8584 & Platinum on Fresh Alumina (Pt 0.540 Wt \%) & $25 \mathrm{gm}$ \\
RM8586 & FCC Catalyst & $10 \mathrm{gm}$ \\
RM8587 & FCC Catalyst & $25 \mathrm{gm}$ \\
RM8588 & Faujasite Y & Set of $6,50 \mathrm{gm}$ each \\
RM8589 & FCC Catalysts & \\
\hline
\end{tabular}




\section{Fertilizers}

These SRM's are intended for use in the fertilizer industry as working standards for the determination of the certified constituents.

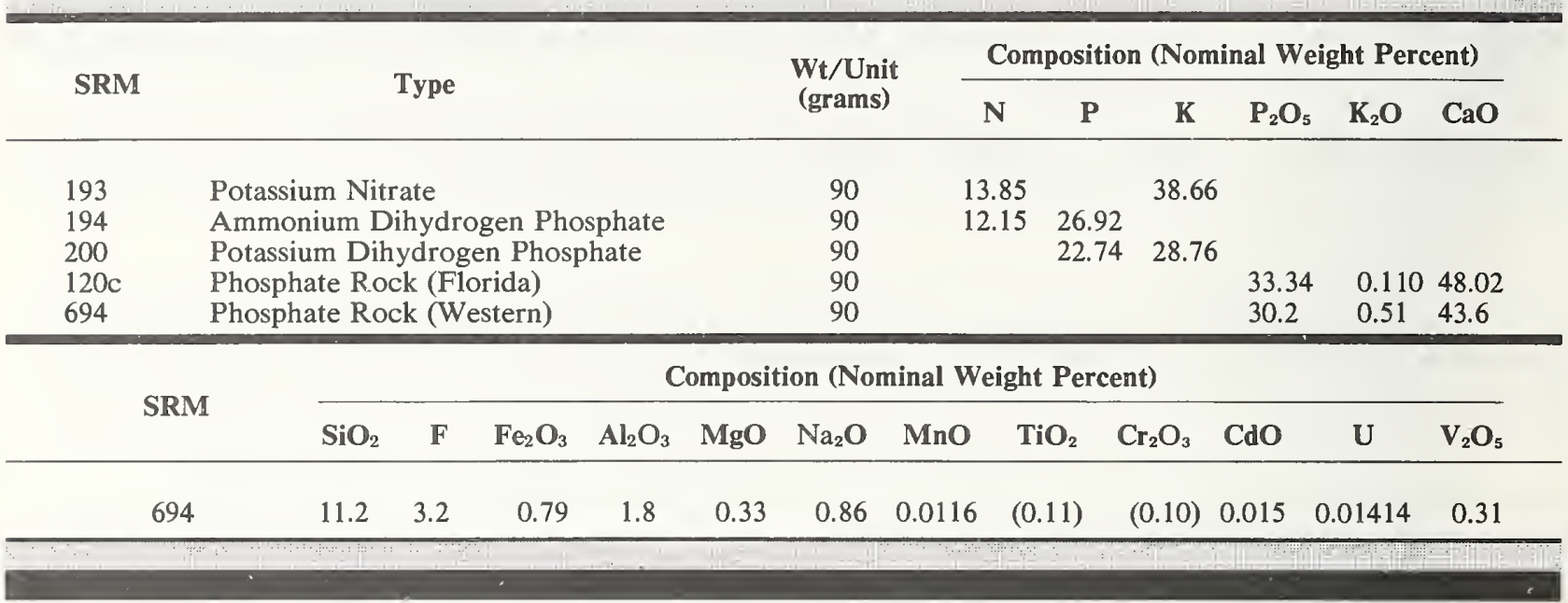

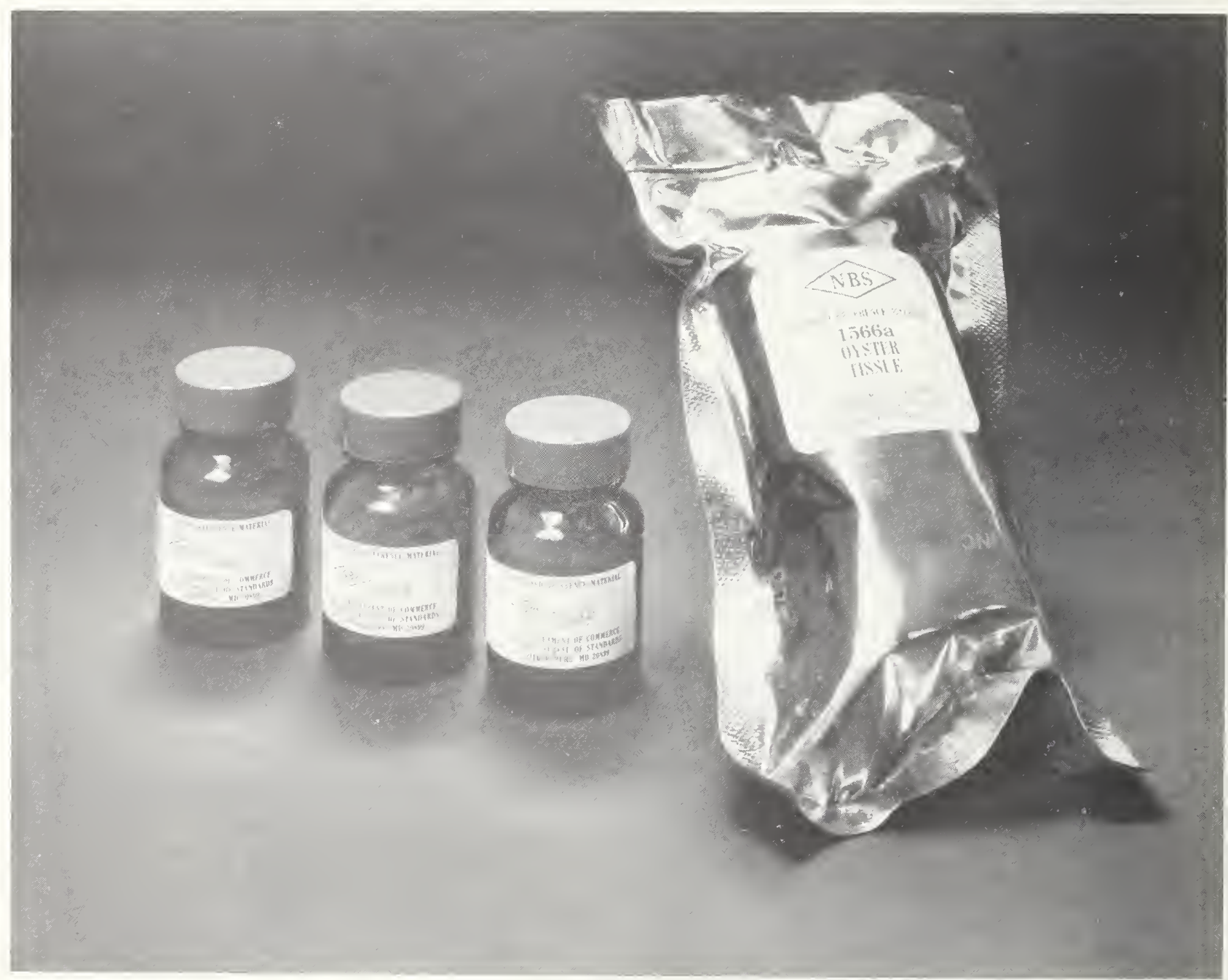

SRM's 1845, Cholesterol in Egg Powder and 1566a, Oyster Tissue are two of the important and popular food related SRM's now available. 


\section{Ores}

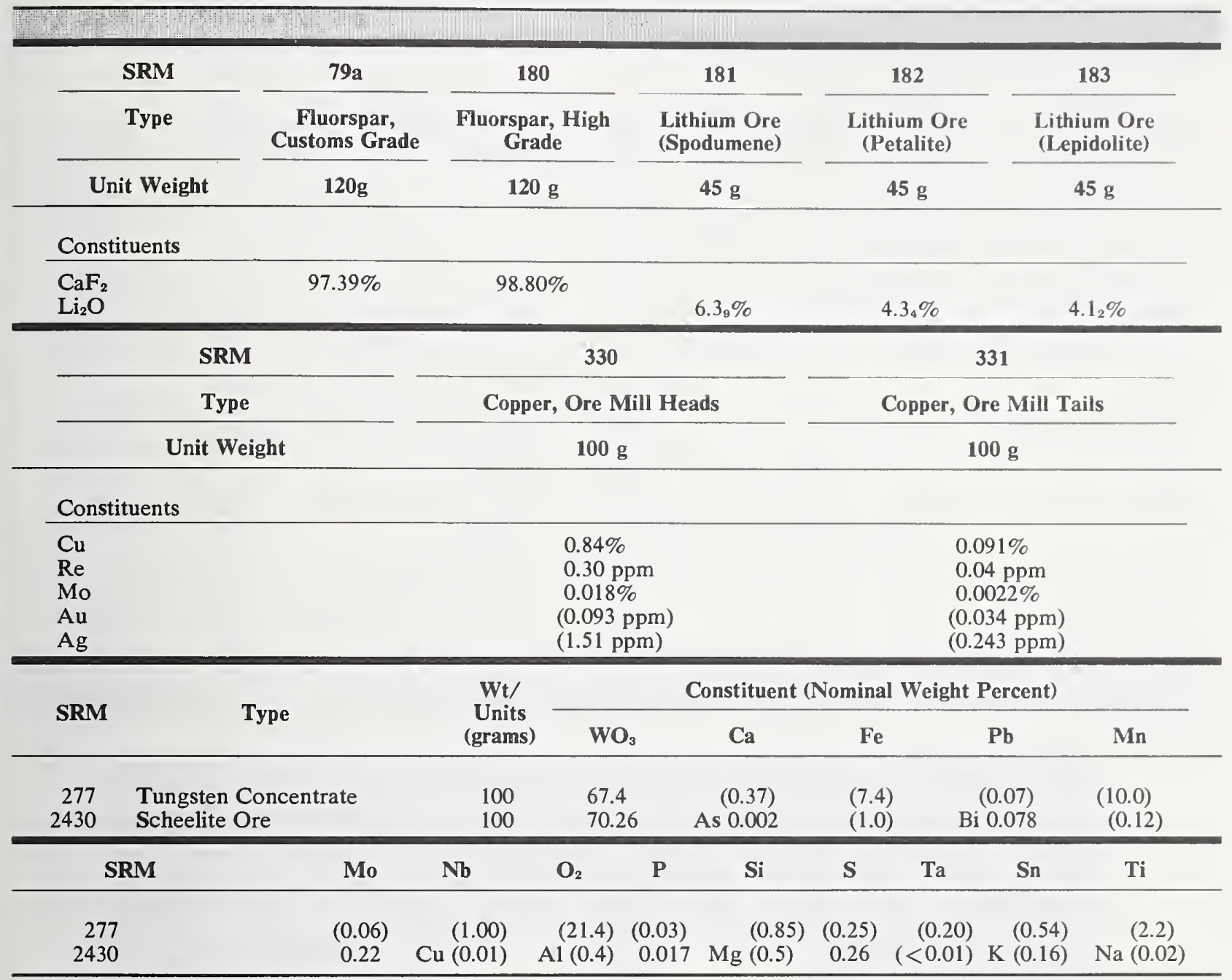

Values in parentheses are not certified, but are given for information only. 


\section{Ores (Continued)}

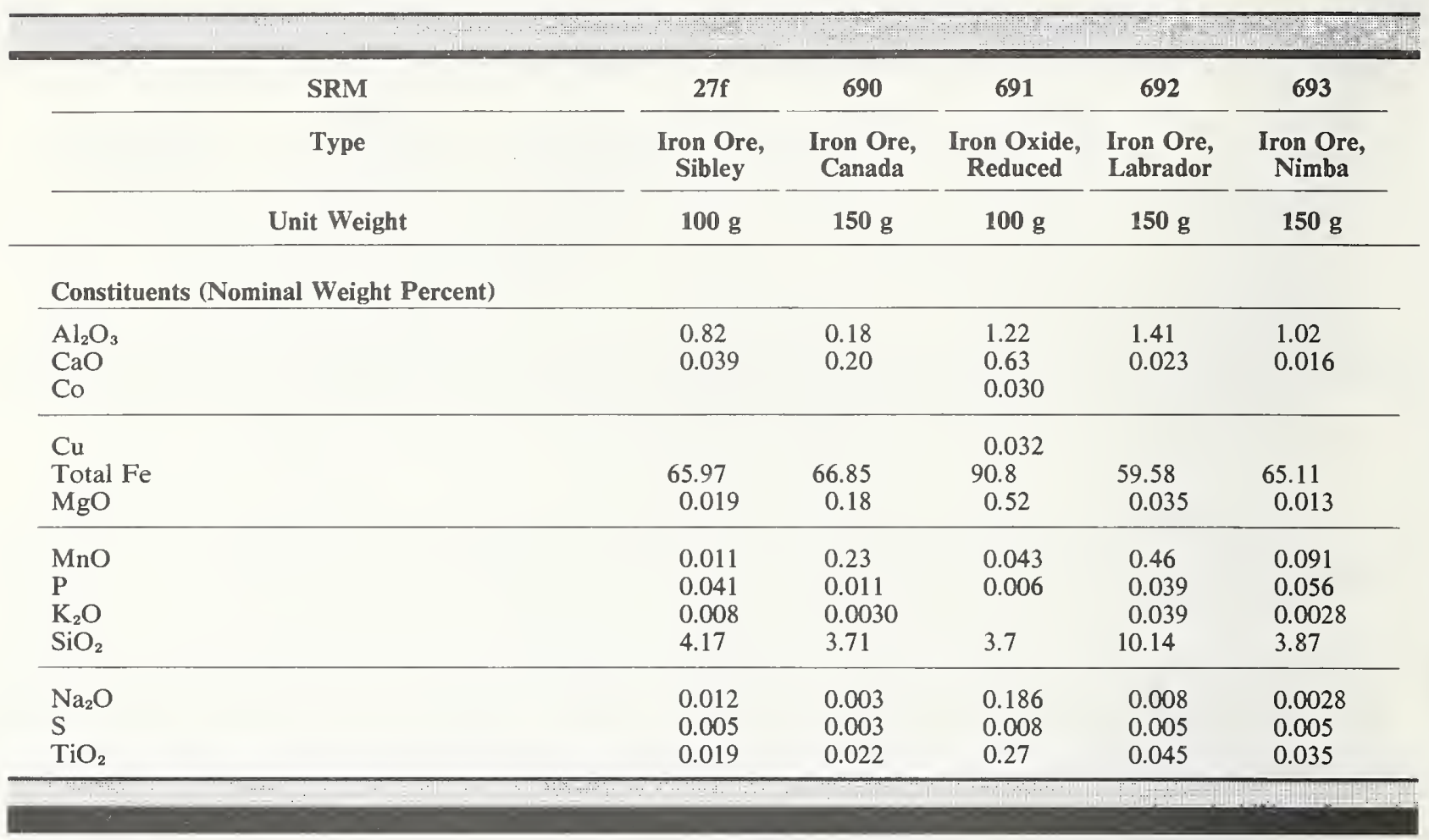

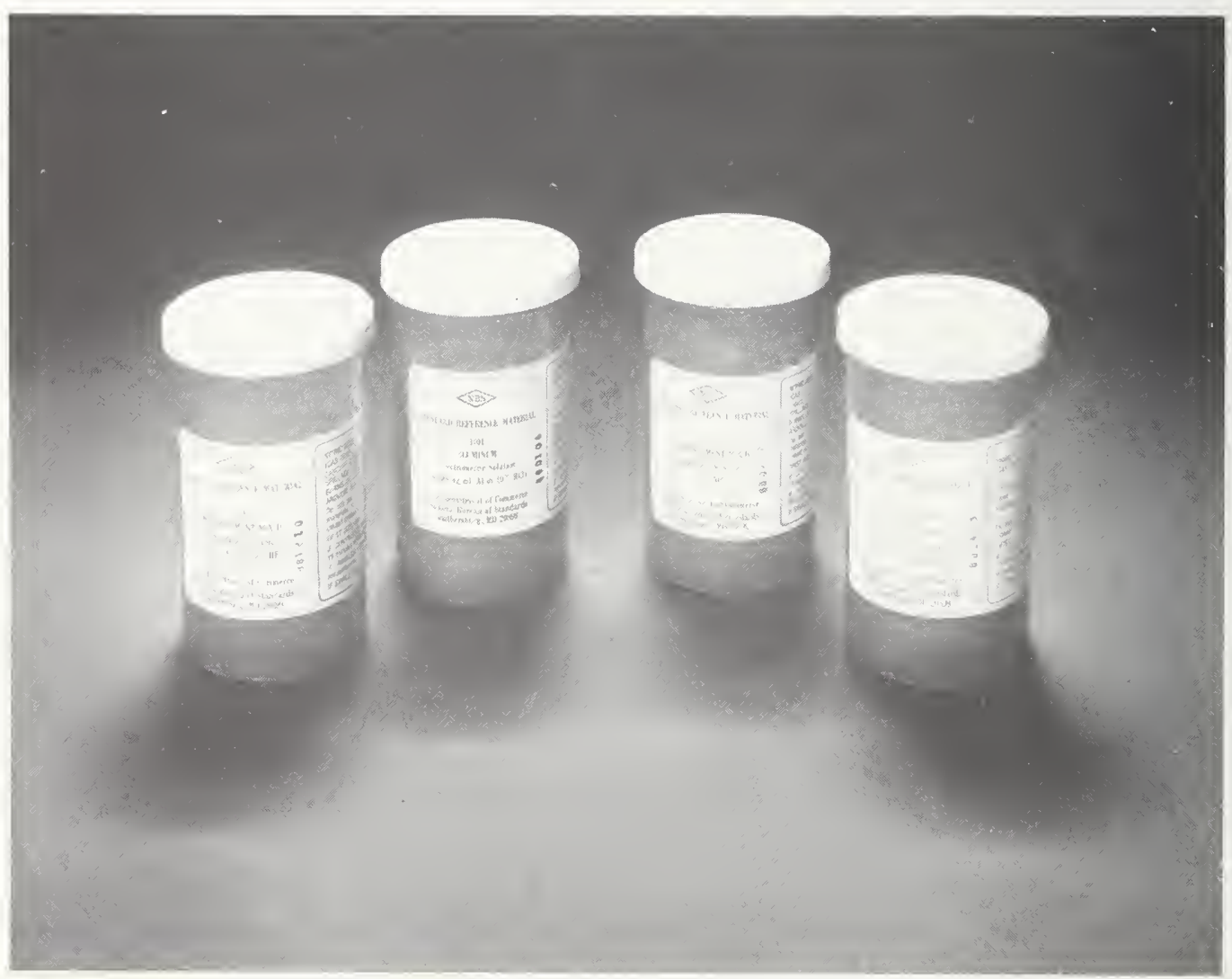

Pictured here are four of the more than 70 spectrometric standard solutions now available either as single element or multielement solutions. 


\section{Ores (Continued)}

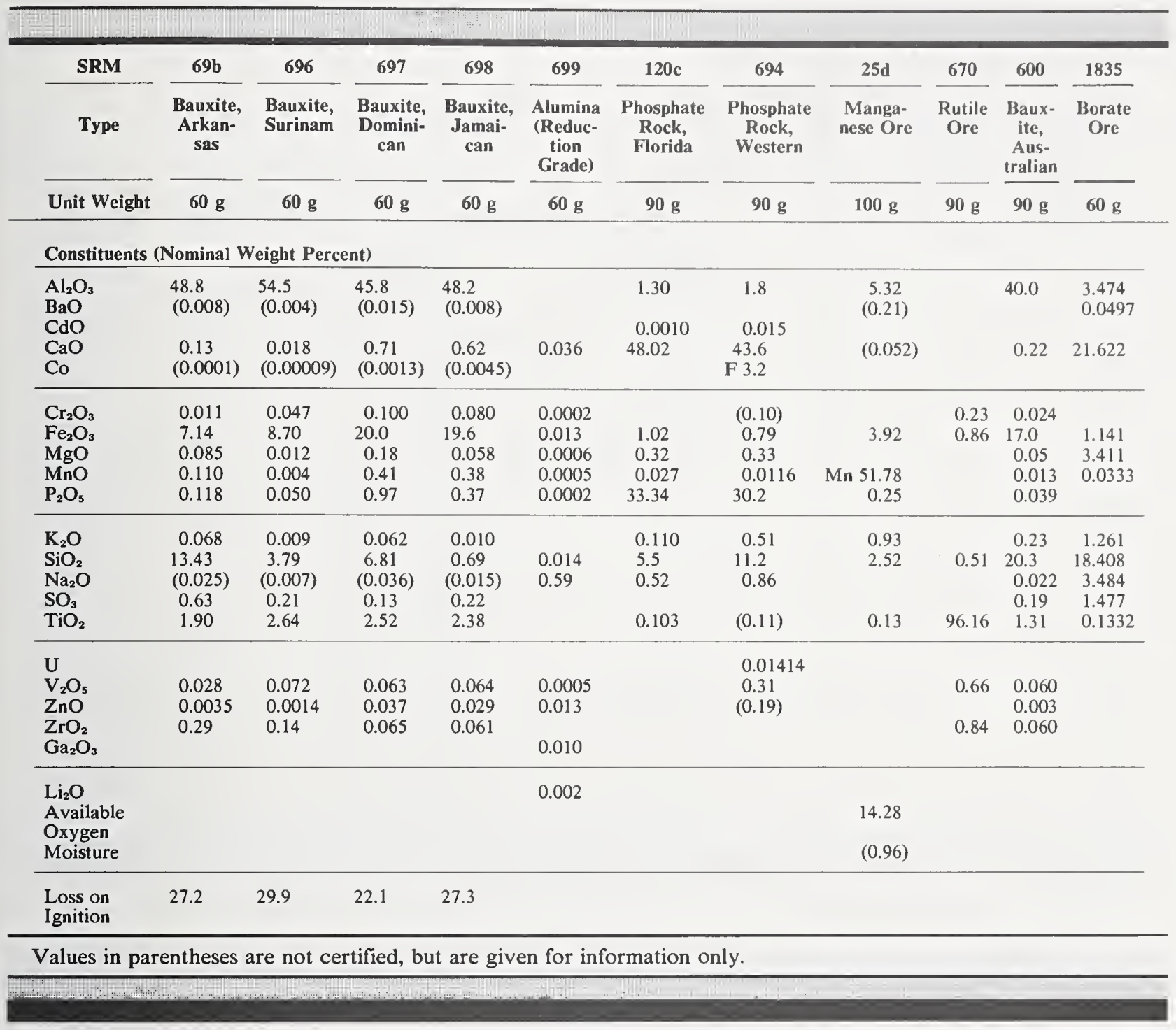




\section{Clays}

\begin{tabular}{|c|c|c|c|}
\hline SRM & $97 \mathrm{~b}$ & $98 \mathrm{~b}$ & 679 \\
\hline Type & Flint Clay & Plastic Clay & Brick Clay \\
\hline Unit Weight & $60 \mathrm{~g}$ & $60 \mathrm{~g}$ & $75 \mathrm{~g}$ \\
\hline $\begin{array}{l}\text { Element } \\
\mathrm{Al} \\
\mathrm{Ba} \\
\mathrm{Ca} \\
\mathrm{Ce} \\
\mathrm{Cs}\end{array}$ & $\begin{array}{l}\text { Nominal Co } \\
20.76 \text { wt. \% } \\
(0.018) \text { wt. \% } \\
0.0249 \text { wt. \% } \\
\text { (3.4) }\end{array}$ & $\begin{array}{l}\text { ons }(\mu \mathrm{g} / \mathrm{g} \text {, unless } \mathrm{n} \\
14.30 \text { wt. } \% \\
(0.07) \text { wt. } \% \\
0.0759 \text { wt } \% \\
(16.5)\end{array}$ & $\begin{array}{l}\text { herwise) } \\
\qquad \begin{array}{l}11.01 \text { wt. \% } \\
0.1628 \text { wt. \% } \\
(105) \\
(9.6)\end{array}\end{array}$ \\
\hline $\begin{array}{l}\mathrm{Cr} \\
\mathrm{Co} \\
\mathrm{Eu} \\
\mathrm{Hf} \\
\mathrm{Fe}\end{array}$ & $\begin{array}{l}227 \\
(3.8) \\
(0.84) \\
(13) \\
0.831 \text { wt. \% }\end{array}$ & $\begin{array}{l}119 \\
(16.3) \\
(1.3) \\
(7.2) \\
1.18 \text { wt. \% }\end{array}$ & $\begin{array}{l}109.7 \\
(26) \\
(1.9) \\
(4.6) \\
9.05 \text { wt. \% } \\
\end{array}$ \\
\hline $\begin{array}{l}\mathrm{Li} \\
\mathrm{Mg} \\
\mathrm{Mn} \\
\mathrm{P} \\
\mathrm{K}\end{array}$ & $\begin{array}{l}550 \\
0.113 \text { wt. \% } \\
47 \\
(200) \\
0.513 \text { wt. \% }\end{array}$ & $\begin{array}{l}215 \\
0.358 \text { wt. } \% \\
116 \\
(300) \\
2.81 \text { wt. } \%\end{array}$ & $\begin{array}{l}71.7 \\
0.7552 \text { wt. \% } \\
(1730) \\
(750) \\
2.433 \text { wt. \% }\end{array}$ \\
\hline $\begin{array}{l}\mathrm{Rb} \\
\mathrm{Sc} \\
\mathrm{Si} \\
\mathrm{Na} \\
\mathrm{Sr}\end{array}$ & $\begin{array}{l}(33) \\
(22) \\
19.81 \text { wt. } \% \\
0.0492 \text { wt. \% } \\
84\end{array}$ & $\begin{array}{l}(180) \\
(22) \\
26.65 \text { wt. \% } \\
0.1496 \text { wt. \% } \\
189\end{array}$ & $\begin{array}{l}(190) \\
(22.5) \\
24.34 \text { wt. \% } \\
0.1304 \text { wt. \% } \\
73.4 \\
\end{array}$ \\
\hline $\begin{array}{l}\mathrm{Th} \\
\mathrm{Ti} \\
\mathrm{Zn} \\
\mathrm{Zr} \\
\mathrm{Sb}\end{array}$ & $\begin{array}{l}(36) \\
1.43 \text { wt. \% } \\
(87) \\
(0.05) \text { wt. \% } \\
(2.2)\end{array}$ & $\begin{array}{l}(21) \\
0.809 \text { wt. \% } \\
(110) \\
(0.022) \text { wt. \% } \\
(1.6)\end{array}$ & $\begin{array}{l}(14) \\
0.577 \text { wt. \% } \\
(150)\end{array}$ \\
\hline $\begin{array}{l}\text { Loss on } \\
\text { Ignition }\end{array}$ & (13.3 wt. \%) & (7.5 wt. \%) & \\
\hline
\end{tabular}

Values in parentheses are not certified, but are given for information only.

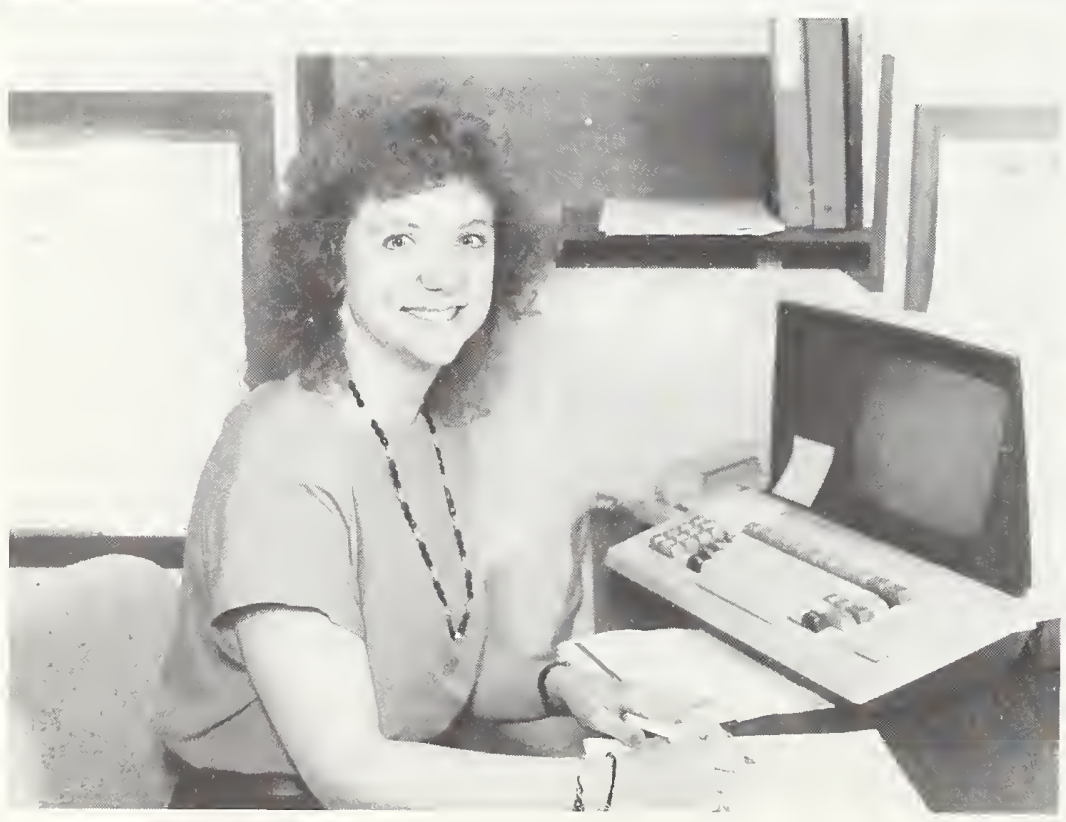

Karen Applestein provides the alertness and attention to detail that ensures that each customers order or service request is handled punctually and accurately. 


\section{Rocks, Minerals, and Refractories}

\begin{tabular}{|c|c|c|c|c|c|c|c|c|c|}
\hline SRM & 1c & $88 b$ & $70 \mathbf{a}$ & $99 \mathrm{a}$ & $81 a$ & $165 a$ & 1413 & 2709 & 2710 \\
\hline Type & $\begin{array}{c}\text { Lime- } \\
\text { stone, } \\
\text { argilla- } \\
\text { ceous } \\
\end{array}$ & $\begin{array}{l}\text { Limestone, } \\
\text { dolomitic }\end{array}$ & $\begin{array}{l}\text { Feld- } \\
\text { spar, } \\
\text { potash }\end{array}$ & $\begin{array}{l}\text { Feld- } \\
\text { spar, } \\
\text { soda }\end{array}$ & $\begin{array}{l}\text { Glass } \\
\text { sand }\end{array}$ & $\begin{array}{l}\text { Glass } \\
\text { sand } \\
\text { (low } \\
\text { iron) }\end{array}$ & $\begin{array}{l}\text { Glass } \\
\text { sand } \\
\text { (high } \\
\text { alumina) }\end{array}$ & $\begin{array}{l}\text { Agricultur- } \\
\text { al Soil } \\
\text { (Baseline } \\
\text { Levels) }\end{array}$ & $\begin{array}{l}\text { Agricultur- } \\
\text { al Soil } \\
\text { (Elevated } \\
\text { Levels) }\end{array}$ \\
\hline $\begin{array}{l}\text { Unit } \\
\text { Weight }\end{array}$ & $50 \mathrm{~g}$ & $75 \mathrm{~g}$ & $40 \mathrm{~g}$ & $40 \mathrm{~g}$ & $75 \mathrm{~g}$ & $75 \mathrm{~g}$ & $75 \mathrm{~g}$ & IN PREP & IN PREP \\
\hline
\end{tabular}

Constituents (Nominal Weight Percent)

\begin{tabular}{|c|c|c|c|c|c|c|c|}
\hline $\begin{array}{l}\mathrm{Al}_{2} \mathrm{O}_{3} \\
\mathrm{BaO} \\
\mathrm{CaO} \\
\mathrm{Cr}_{2} \mathrm{O}_{3} \\
\mathrm{Fe}_{2} \mathrm{O}_{3}\end{array}$ & $\begin{array}{l}1.30 \\
50.3 \\
0.55\end{array}$ & 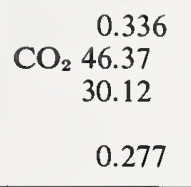 & $\begin{array}{c}17.9 \\
0.02 \\
0.11 \\
\\
0.07_{5}\end{array}$ & $\begin{array}{c}20.5 \\
0.26 \\
2.14 \\
\\
0.06_{5}\end{array}$ & $\begin{array}{l}0.66 \\
46 \mu \mathrm{g} / \mathrm{g} \\
0.082\end{array}$ & $\begin{array}{l}\text { (1) } \mu \mathrm{g} / \mathrm{g} \\
0.012\end{array}$ & $\begin{array}{l}9.90 \\
0.12 \\
0.74 \\
\\
0.24\end{array}$ \\
\hline $\begin{array}{l}\mathrm{MgO} \\
\mathrm{MnO} \\
\mathrm{P}_{2} \mathrm{O}_{5} \\
\mathrm{~K}_{2} \mathrm{O}\end{array}$ & $\begin{array}{l}0.42 \\
0.025 \\
0.04 \\
0.28\end{array}$ & $\begin{array}{l}21.03 \\
0.0160 \\
0.0044 \\
0.1030\end{array}$ & 11.8 & $\begin{array}{l}0.02 \\
\\
0.02 \\
5.2\end{array}$ & & & 0.06 \\
\hline $\begin{array}{l}\mathrm{Rb}_{2} \mathrm{O} \\
\mathrm{SiO}_{2} \\
\mathrm{Na}_{2} \mathrm{O} \\
\mathrm{SrO} \\
\mathrm{TiO}_{2} \\
\mathrm{ZrO}_{2}\end{array}$ & $\begin{array}{l}6.84 \\
0.02 \\
0.030 \\
0.07\end{array}$ & $\begin{array}{l}1.13 \\
0.0290 \\
0.0076 \\
(0.016)\end{array}$ & $\begin{array}{c}0.06 \\
67.1 \\
2.5_{5} \\
\\
0.01\end{array}$ & $\begin{array}{l}65.2 \\
6.2 \\
0.007\end{array}$ & $\begin{array}{l}0.12 \\
0.034\end{array}$ & $\begin{array}{l}0.011 \\
0.006\end{array}$ & $\begin{array}{r}82.77 \\
1.75\end{array}$ \\
\hline
\end{tabular}

\begin{tabular}{|c|c|c|}
\hline Coss on & 39.9 & $(46.98)$ \\
\hline
\end{tabular}
Ignition

Values in parentheses are not certified, but are given for information only.

\section{Mercury in Soil}

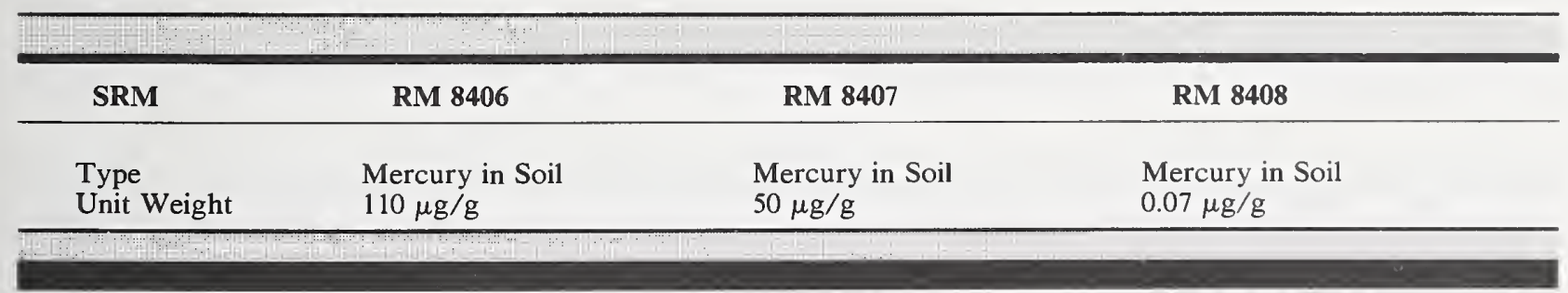




\section{Rocks, Minerals, and Refractories (Continued)}

\begin{tabular}{|c|c|c|c|c|c|c|}
\hline SRM & $154 b$ & 278 & 688 & $76 \mathrm{a}$ & $77 \mathrm{a}$ & $78 \mathrm{a}$ \\
\hline Type & $\begin{array}{l}\text { Titanium } \\
\text { Dioxide }\end{array}$ & Obsidian Rock & $\begin{array}{l}\text { Basalt } \\
\text { Rock }\end{array}$ & $\begin{array}{c}\text { Burnt } \\
\text { Refractory } \\
\left(\mathrm{Al}_{2} \mathrm{O}_{3}-40 \%\right) \\
\end{array}$ & $\begin{array}{c}\text { Burnt } \\
\text { Refractory } \\
\left(\mathrm{Al}_{2} \mathrm{O}_{3}-60 \%\right) \\
\end{array}$ & $\begin{array}{c}\text { Burnt } \\
\text { Refractory } \\
\left(\mathrm{Al}_{2} \mathrm{O}_{3}-70 \%\right) \\
\end{array}$ \\
\hline $\begin{array}{c}\text { Unit } \\
\text { Weight }\end{array}$ & $90 \mathrm{~g}$ & $35 \mathrm{~g}$ & $60 \mathrm{~g}$ & $75 \mathrm{~g}$ & $75 \mathrm{~g}$ & $75 \mathrm{~g}$ \\
\hline \multicolumn{7}{|c|}{ Constituents (Nominal Weight Percent) } \\
\hline $\begin{array}{l}\mathrm{Al}_{2} \mathrm{O}_{3} \\
\mathrm{CaO} \\
\mathrm{Cr} \\
\mathrm{Cu} \\
\mathrm{FeO}\end{array}$ & $(\sim 0.01)$ & $\begin{array}{l}14.15 \\
0.983 \\
\\
5.9 \mu \mathrm{g} / \mathrm{g} \\
1.36\end{array}$ & $\begin{array}{l}17.36 \\
(12.17) \\
332 \mu \mathrm{g} / \mathrm{g} \\
7.64\end{array}$ & $\begin{array}{c}38.7 \\
0.22\end{array}$ & $\begin{array}{c}60.2 \\
0.05\end{array}$ & $\begin{array}{c}71.7 \\
0.11\end{array}$ \\
\hline $\begin{array}{l}\mathrm{Fe}_{2} \mathrm{O}_{3} \\
\mathrm{~Pb} \\
\mathrm{Li}_{2} \mathrm{O} \\
\mathrm{MgO} \\
\mathrm{MnO} \\
\mathrm{Ni}\end{array}$ & $\begin{array}{r}(0.006) \\
(\sim 0.01)\end{array}$ & $\begin{array}{l}2.04 \\
16.4 \mu \mathrm{g} / \mathrm{g} \\
\\
(0.23) \\
0.052 \\
3.6 \mu \mathrm{g} / \mathrm{g}\end{array}$ & $\begin{array}{l}10.35 \\
3.3 \mu \mathrm{g} / \mathrm{g} \\
(8.4) \\
0.167\end{array}$ & $\begin{array}{l}1.60 \\
0.042 \\
0.52\end{array}$ & $\begin{array}{l}1.0_{0} \\
0.02_{5} \\
0.38\end{array}$ & $\begin{array}{l}1.2 \\
0.12 \\
0.70\end{array}$ \\
\hline $\begin{array}{l}\mathrm{P}_{2} \mathrm{O}_{5} \\
\mathrm{~K}_{2} \mathrm{O} \\
\mathrm{Rb} \\
\mathrm{SiO}_{2}\end{array}$ & $\begin{array}{l}(0.04) \\
(0.01)\end{array}$ & $\begin{array}{c}0.036 \\
4.16 \\
127.5 \mu \mathrm{g} / \mathrm{g} \\
73.05\end{array}$ & $\begin{array}{l}0.134 \\
0.187 \\
1.91 \mu \mathrm{g} / \mathrm{g} \\
48.4\end{array}$ & $\begin{array}{l}0.12_{0} \\
1.33 \\
54.9\end{array}$ & $\begin{array}{l}0.092 \\
0.090 \\
35.0\end{array}$ & $\begin{array}{c}1.3 \\
1.22 \\
19.4\end{array}$ \\
\hline $\begin{array}{l}\mathrm{Na}_{2} \mathrm{O} \\
\mathrm{Sr} \\
\mathrm{SrO} \\
\mathrm{Th} \\
\mathrm{TiO}_{2}\end{array}$ & 99.74 & $\begin{array}{l}4.84 \\
63.5 \mu \mathrm{g} / \mathrm{g} \\
12.4 \mu \mathrm{g} / \mathrm{g} \\
0.245\end{array}$ & $\begin{array}{l}2.15 \\
169.2 \mu \mathrm{g} / \mathrm{g} \\
0.33 \mu \mathrm{g} / \mathrm{g} \\
1.17\end{array}$ & $\begin{array}{l}0.07 \\
0.037 \\
2.0_{3}\end{array}$ & $\begin{array}{l}0.037 \\
0.009 \\
2.6_{6}\end{array}$ & $\begin{array}{l}0.078 \\
0.25 \\
3.22\end{array}$ \\
\hline $\begin{array}{l}\text { T1 } \\
\text { U } \\
\text { Loss on } \\
\text { Ignition }\end{array}$ & & $\begin{array}{l}0.54 \mu \mathrm{g} / \mathrm{g} \\
4.58 \mu \mathrm{g} / \mathrm{g}\end{array}$ & & $(0.34)$ & $(0.22)$ & $(0.42)$ \\
\hline
\end{tabular}

Values in parentheses are not certified, but are given for information only. 


\section{Rocks, Minerals, and Refractories (Continued)}

\begin{tabular}{|c|c|c|c|}
\hline SRM & $103 a$ & 198 & 199 \\
\hline Type & Chrome Refractory & Silica Brick & Silica Brick \\
\hline Unit Weight & $60 \mathrm{~g}$ & $45 \mathrm{~g}$ & $45 \mathrm{~g}$ \\
\hline \multicolumn{4}{|c|}{ Constituents (Nominal Weight Percent) } \\
\hline $\begin{array}{l}\mathrm{Al}_{2} \mathrm{O}_{3} \\
\mathrm{CaO} \\
\mathrm{Cr}_{2} \mathrm{O}_{3} \\
\mathrm{FeO} \\
\mathrm{Fe}_{2} \mathrm{O}_{3} \\
\end{array}$ & $\begin{array}{r}29.96 \\
0.69 \\
32.06 \\
12.43\end{array}$ & $\begin{array}{l}0.16 \\
2.71 \\
\\
0.66\end{array}$ & $\begin{array}{l}0.48 \\
2.41\end{array}$ \\
\hline $\begin{array}{l}\mathrm{Li}_{2} \mathrm{O} \\
\mathrm{MgO} \\
\mathrm{MnO} \\
\mathrm{P}_{2} \mathrm{O}_{5} \\
\mathrm{~K}_{2} \mathrm{O}\end{array}$ & $\begin{array}{r}18.54 \\
0.11 \\
0.01\end{array}$ & $\begin{array}{l}0.001 \\
0.07 \\
0.008 \\
0.022 \\
0.017\end{array}$ & $\begin{array}{l}0.002 \\
0.13 \\
0.007 \\
0.015 \\
0.094\end{array}$ \\
\hline $\begin{array}{l}\mathrm{SiO}_{2} \\
\mathrm{Na}_{2} \mathrm{O} \\
\mathrm{TiO}_{2} \\
\mathrm{ZrO}_{2} \\
\text { Loss on Ignition }\end{array}$ & $\begin{array}{l}4.63 \\
\\
0.22 \\
0.01\end{array}$ & $\begin{array}{l}0.012 \\
0.02 \\
0.21\end{array}$ & $\begin{array}{l}0.015 \\
0.06 \\
0.17\end{array}$ \\
\hline
\end{tabular}

Values in parentheses are not certified, but are given for information only.

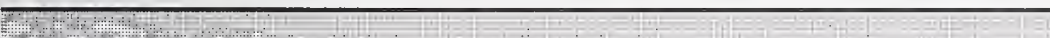
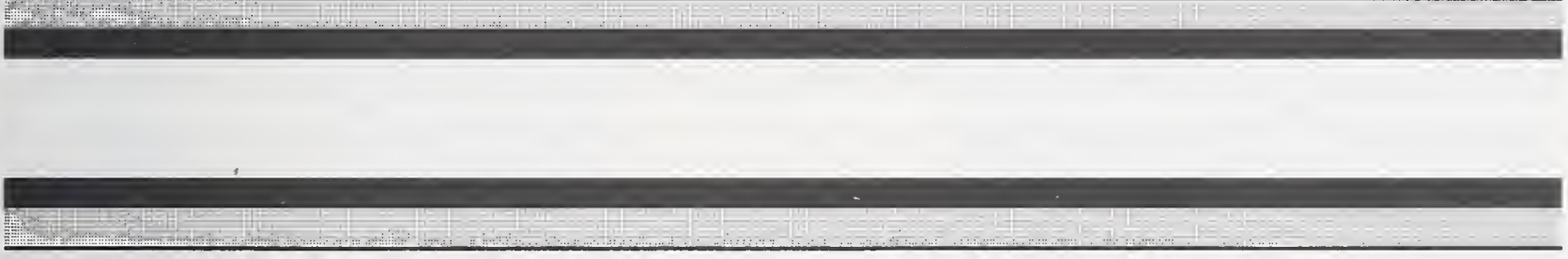

\section{Carbides}

\begin{tabular}{|c|c|c|c|c|c|c|c|c|}
\hline \multirow{2}{*}{ SRM } & \multirow{2}{*}{ Type } & \multirow{2}{*}{$\begin{array}{l}\text { Wt/Unit } \\
\text { (grams) }\end{array}$} & \multicolumn{6}{|c|}{ Chemical Composition (Nominal Weight Percent) } \\
\hline & & & $\mathrm{SiC}$ & Total C & Free C & $\mathbf{F e}$ & $\mathbf{O}_{2}$ & $\mathbf{N}_{2}$ \\
\hline $\begin{array}{l}112 b \\
276 a\end{array}$ & $\begin{array}{l}\text { Silicon Carbide } \\
\text { Tungsten Carbide }\end{array}$ & $\begin{array}{l}80 \\
75\end{array}$ & 97.37 & $\begin{array}{r}29.43 \\
6.11\end{array}$ & $\begin{array}{c}0.26 \\
(0.02)\end{array}$ & 0.13 & $(0.03)$ & $(0.003)$ \\
\hline
\end{tabular}




\section{Cemented Carbides}

SRM's 887-9 are fine powders prepared from sintered Tungsten Carbide bare materials.

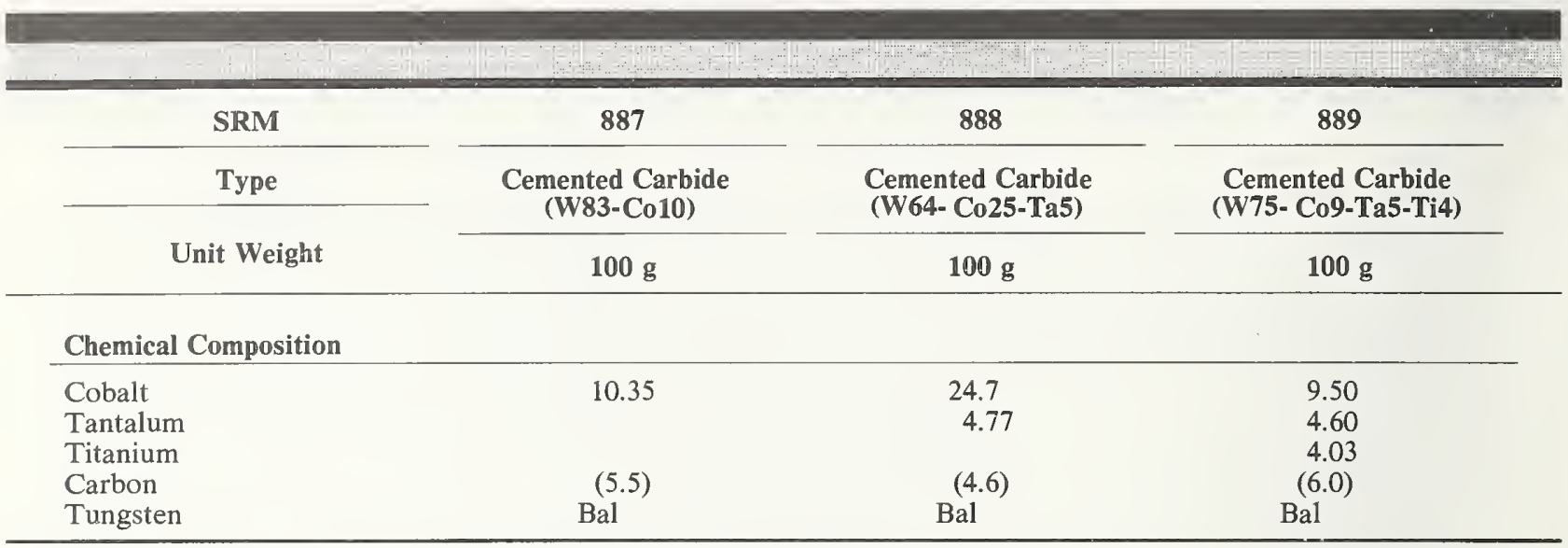

Values in parentheses are not certified, but are given for information only.

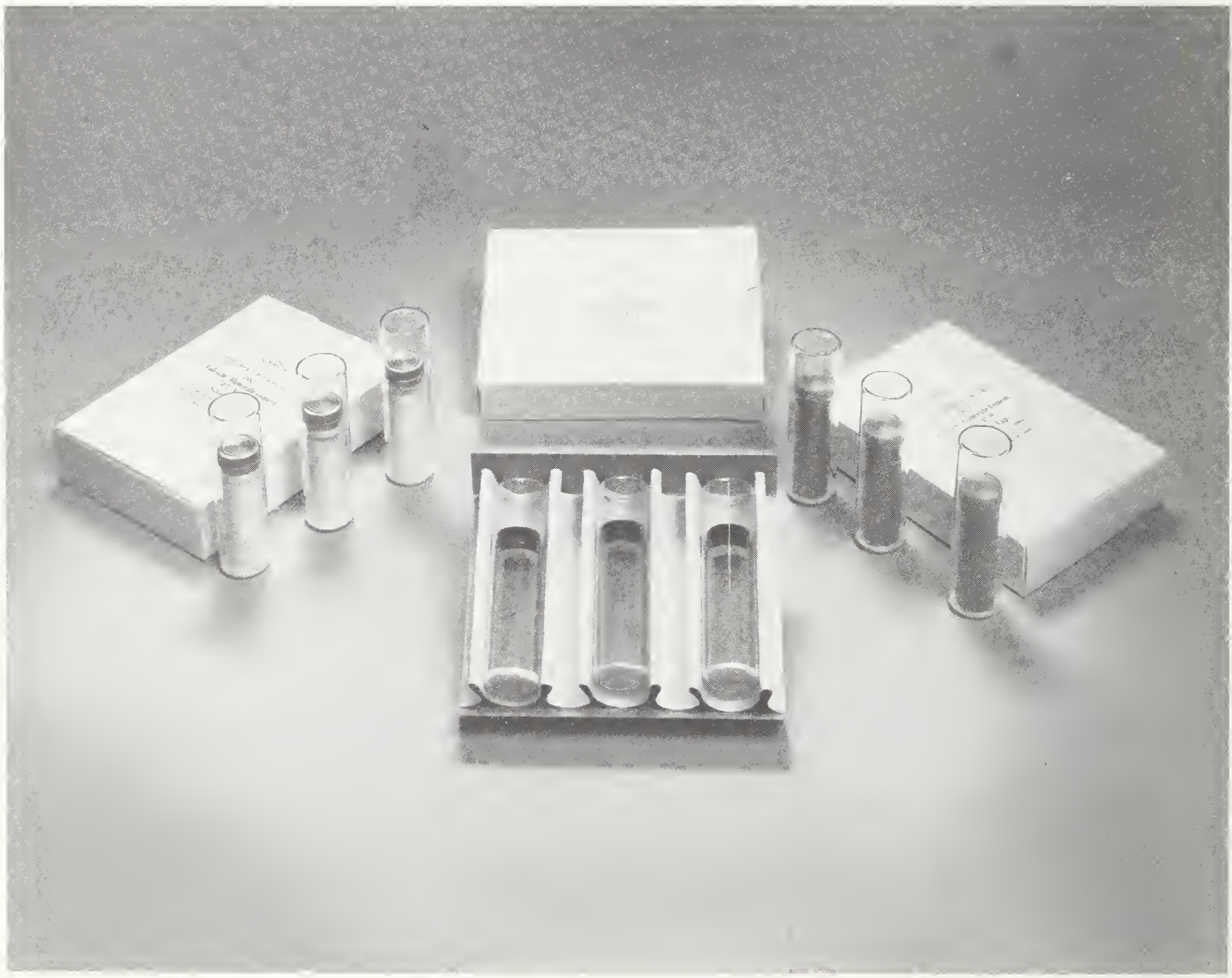

The portland and calcium aluminate SRM's pictured here are recent additions to very popular "630" portland cement composition SRM's. A new series-SRM's 1884-1889-are now available to replace the "630" series as those supplies are exhausted. 


\section{Glasses}

\begin{tabular}{|c|c|c|c|c|c|c|c|c|c|c|c|}
\hline SRM & 89 & 91 & 92 & $93 \mathbf{a}$ & 620 & 621 & 1411 & 1412 & 1830 & 1831 & 1834 \\
\hline \multirow[b]{2}{*}{ Unit Size } & $\begin{array}{c}\text { Lead- } \\
\text { Barium }\end{array}$ & $\begin{array}{c}\text { Opal } \\
\text { Powder }\end{array}$ & $\begin{array}{l}\text { Low- } \\
\text { Boron } \\
\text { Soda- } \\
\text { Lime } \\
\text { Powder }\end{array}$ & $\begin{array}{l}\text { High- } \\
\text { Boron } \\
\text { Boro- } \\
\text { silicate }\end{array}$ & $\begin{array}{l}\text { Soda- } \\
\text { Lime, } \\
\text { Flat }\end{array}$ & $\begin{array}{l}\text { Soda- } \\
\text { Lime, } \\
\text { Con- } \\
\text { tainer }\end{array}$ & $\begin{array}{c}\text { Soft } \\
\text { Boro- } \\
\text { silicate }\end{array}$ & $\begin{array}{c}\text { Multi } \\
\text { Compo- } \\
\text { nent }\end{array}$ & $\begin{array}{l}\text { Soda- } \\
\text { Lime, } \\
\text { Float }\end{array}$ & $\begin{array}{l}\text { Soda- } \\
\text { Lime, } \\
\text { Sheet }\end{array}$ & $\begin{array}{c}\text { Fused } \\
\text { Ore } \\
\text { Glass }\end{array}$ \\
\hline & $45 \mathrm{~g}$ & $45 \mathrm{~g}$ & $45 \mathrm{~g}$ & $\begin{array}{c}\text { Wafer } \\
32 \mathrm{~mm} \\
\mathrm{D} \times 6 \\
\mathrm{~mm}\end{array}$ & $\begin{array}{c}3 \text { platelets } \\
35 \times 35 \times 3 \\
\mathrm{~mm}\end{array}$ & $\begin{array}{c}3 \text { disks } \\
38 \mathrm{~mm} \\
\mathrm{D} \times 5 \\
\mathrm{~mm}\end{array}$ & $\begin{array}{l}10 \\
\text { plate- } \\
\text { lets }\end{array}$ & $\begin{array}{c}8 \\
\text { platelets }\end{array}$ & $\begin{array}{c}3 \\
\text { platelets } \\
38 \times 38 \times \\
6 \mathrm{~mm}\end{array}$ & $\begin{array}{c}3 \\
\text { platelets } \\
37 \times 37 \times \\
3 \mathrm{~mm}\end{array}$ & $\begin{array}{c}\text { IN } \\
\text { PREP }\end{array}$ \\
\hline \multicolumn{12}{|c|}{ Constituent (Nominal Weight Percent) } \\
\hline $\begin{array}{l}\mathrm{SiO}_{2} \\
\mathrm{PbO}\end{array}$ & $\begin{array}{l}65.35 \\
17.50\end{array}$ & $\begin{array}{c}67.5 \\
0.10\end{array}$ & $(75.0)$ & 80.8 & 72.08 & 71.13 & 58.04 & $\begin{array}{r}42.38 \\
4.40\end{array}$ & 73.07 & 73.08 & \\
\hline $\mathrm{Al}_{2} \mathrm{O}_{3}$ & 0.18 & 6.01 & & $2.2_{8}$ & 1.80 & 2.76 & 5.68 & 7.52 & 0.12 & 1.21 & \\
\hline $\begin{array}{l}\mathrm{Fe}_{2} \mathrm{O}_{3} \\
\mathrm{ZnO}\end{array}$ & 0.049 & $\begin{array}{l}0.079 \\
0.08\end{array}$ & $(0.2)$ & 0.028 & 0.043 & 0.040 & $\begin{array}{l}0.050 \\
3.85\end{array}$ & $\begin{array}{c}(0.031) \\
4.48\end{array}$ & 0.121 & 0.087 & \\
\hline $\begin{array}{l}\mathrm{CdO} \\
\mathrm{MnO}\end{array}$ & 0.088 & $(0.008)$ & & & & & & 4.38 & & & \\
\hline $\mathrm{TiO}_{2}$ & 0.01 & 0.019 & & $0.01_{4}$ & 0.018 & 0.014 & 0.02 & & 0.011 & 0.019 & \\
\hline $\mathrm{ZrO}_{2}$ & 0.005 & 0.009 & & $0.04_{2}$ & & 0.007 & & & & & \\
\hline $\mathrm{CaO}$ & 0.21 & 10.49 & $(8.3)$ & 0.01 & 7.11 & 10.71 & 2.18 & 4.53 & 8.56 & 8.20 & \\
\hline $\begin{array}{l}\mathrm{BaO} \\
\mathrm{Li}_{2} \mathrm{O}\end{array}$ & 1.40 & & & & & 0.12 & 5.00 & $\begin{array}{c}4.67 \\
(4.50)\end{array}$ & & • & \\
\hline $\mathrm{MgO}$ & 0.03 & $(0.008)$ & $(0.1)$ & $0.00_{5}$ & 3.69 & 0.27 & 0.33 & (4.69) & 3.90 & 3.51 & \\
\hline $\mathrm{K}_{2} \mathrm{O}$ & 8.40 & 3.24 & $(0.6)$ & $0.01_{4}$ & 0.41 & 2.01 & 2.97 & 4.14 & 0.04 & 0.33 & \\
\hline $\mathrm{Na}_{2} \mathrm{O}$ & 5.70 & 8.47 & (13.1) & $3.9_{8}$ & 14.39 & 12.74 & 10.14 & 4.69 & 13.75 & 13.32 & \\
\hline $\begin{array}{l}\mathrm{B}_{2} \mathrm{O}_{3} \\
\mathrm{P}_{2} \mathrm{O}_{5}\end{array}$ & 0.23 & 0.023 & 0.70 & $12.5_{6}$ & & & 10.94 & 4.53 & & & \\
\hline $\mathrm{As}_{2} \mathrm{O}_{5}$ & 0.36 & 0.10 & & & & & & & & & \\
\hline $\mathrm{As}_{2} \mathrm{O}_{3}$ & 0.03 & 0.09 & & & 0.056 & 0.030 & & & & & \\
\hline $\mathrm{SO}_{3}$ & 0.03 & & & & 0.28 & 0.13 & & & 0.26 & 0.25 & \\
\hline $\mathrm{Cl}$ & 0.05 & 0.015 & & $0.06_{0}$ & & & & & & & \\
\hline SrO & & & & & & & 0.09 & 4.55 & & & \\
\hline $\begin{array}{l}\text { F } \\
\text { Loss on }\end{array}$ & & 5.73 & & & & & & & & & \\
\hline Ignition & 0.32 & & $(0.42)$ & & & & & & & & \\
\hline
\end{tabular}

Values in parentheses are not certified, but are given for information only. 


\section{Cements}

These SRM's are for X-ray spectroscopic and chemical analysis of portland cements and related materials. Each unit consists of three sealed vials each containing approximately $5 \mathrm{~g}$ of material.

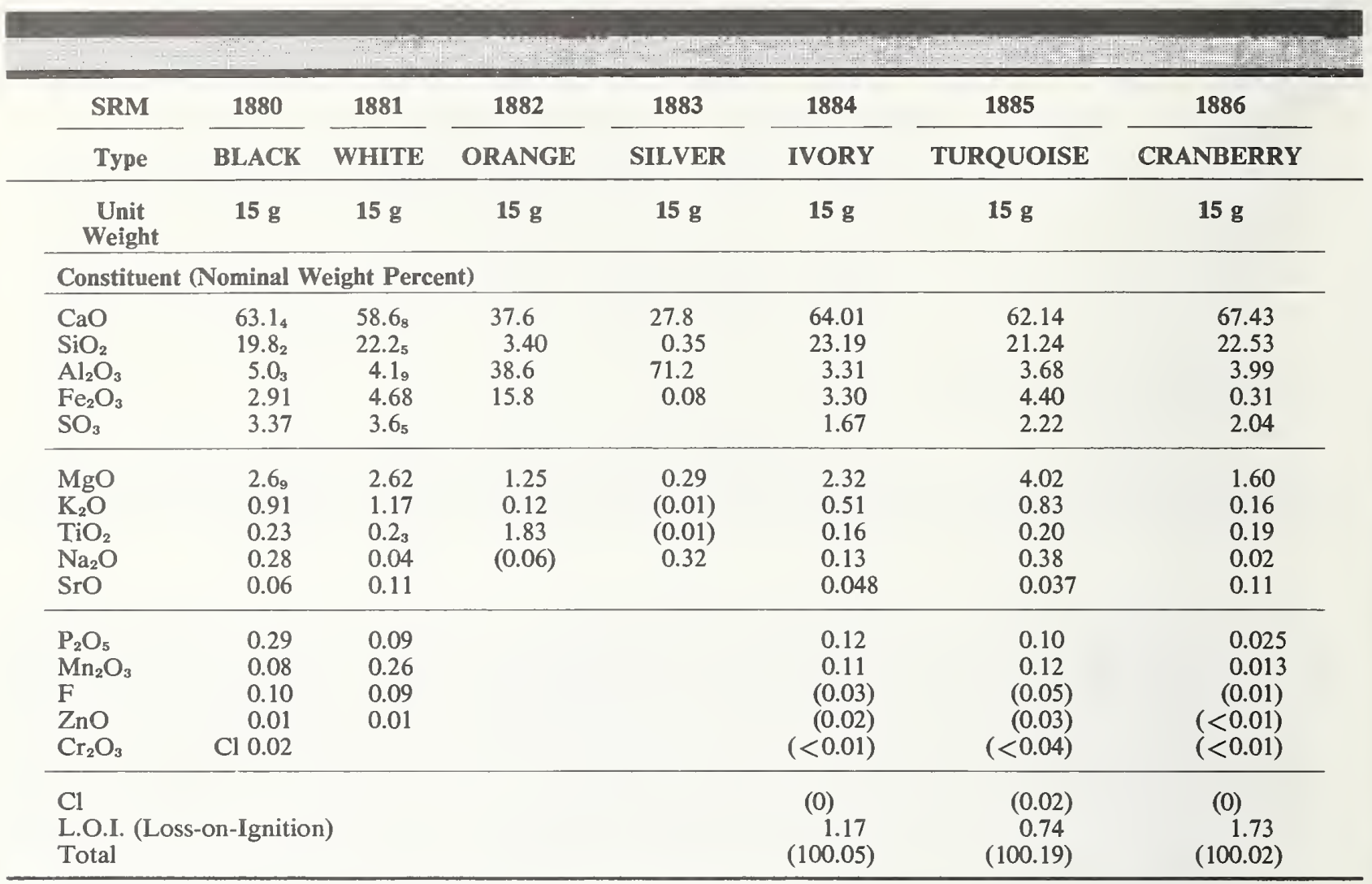

Values in parentheses are not certified, but are given for information only. 


\section{Cements (continued)}

\begin{tabular}{|c|c|c|c|c|c|c|}
\hline SRM & 1887 & 1888 & 1889 & 8486 & 8487 & 8488 \\
\hline Type & BROWN & PURPLE & GRAY & $\begin{array}{l}\text { Portland } \\
\text { Cement } \\
\text { Clinker }\end{array}$ & $\begin{array}{l}\text { Portland } \\
\text { Cement } \\
\text { Clinker }\end{array}$ & $\begin{array}{c}\text { Portland } \\
\text { Cement } \\
\text { Clinker }\end{array}$ \\
\hline Unit Weight & $15 \mathrm{~g}$ & $15 \mathrm{~g}$ & $15 \mathrm{~g}$ & $3 \times 10 \mathrm{~g}$ & $3 \times 10 \mathrm{~g}$ & $3 \times 10 \mathrm{~g}$ \\
\hline \multicolumn{7}{|c|}{ Constituent (Nominal Weight Percent) } \\
\hline $\begin{array}{l}\mathrm{CaO} \\
\mathrm{SiO}_{2} \\
\mathrm{Al}_{2} \mathrm{O}_{3} \\
\mathrm{Fe}_{2} \mathrm{O}_{3} \\
\mathrm{SO}_{3}\end{array}$ & $\begin{array}{r}62.88 \\
19.98 \\
5.59 \\
2.16 \\
4.61 \\
\end{array}$ & $\begin{array}{r}63.78 \\
20.86 \\
5.35 \\
3.18 \\
3.16\end{array}$ & $\begin{array}{r}65.08 \\
20.44 \\
5.61 \\
2.67 \\
2.68\end{array}$ & $\begin{array}{r}(63.36) \\
(22.48) \\
(4.70) \\
(3.60) \\
(0.27)\end{array}$ & $\begin{array}{r}(67.20) \\
(21.43) \\
(5.53) \\
(1.98) \\
(0.88)\end{array}$ & $\begin{array}{r}(66.50) \\
(22.68) \\
(4.90) \\
(4.07) \\
(0.31)\end{array}$ \\
\hline $\begin{array}{l}\mathrm{MgO} \\
\mathrm{K}_{2} \mathrm{O} \\
\mathrm{TiO}_{2} \\
\mathrm{Na}_{2} \mathrm{O} \\
\mathrm{SrO}\end{array}$ & $\begin{array}{l}1.26 \\
1.27 \\
0.27 \\
0.10 \\
0.07\end{array}$ & $\begin{array}{l}0.71 \\
0.56 \\
0.29 \\
0.14 \\
0.07\end{array}$ & $\begin{array}{l}1.38 \\
0.32 \\
0.21 \\
0.11 \\
0.20\end{array}$ & $\begin{array}{l}(4.73) \\
(0.42) \\
(0.25) \\
(0.10) \\
(0.05)\end{array}$ & $\begin{array}{l}(1.48) \\
(0.72) \\
(0.27) \\
(0.14) \\
(0.11)\end{array}$ & $\begin{array}{l}(0.98) \\
(0.35) \\
(0.24) \\
(0.11) \\
(0.13)\end{array}$ \\
\hline $\begin{array}{l}\mathrm{P}_{2} \mathrm{O}_{5} \\
\mathrm{Mnn}_{2} \mathrm{O}_{3} \\
\mathrm{~F} \\
\mathrm{ZnO} \\
\mathrm{Cr}_{2} \mathrm{O}_{3}\end{array}$ & $\begin{array}{c}0.075 \\
0.072 \\
(0.11) \\
(0.01) \\
(<0.01)\end{array}$ & $\begin{array}{l}0.085 \\
0.025 \\
(0.02) \\
(0.01) \\
(0.01)\end{array}$ & $\begin{array}{r}0.15 \\
0.24 \\
(0.04) \\
(<0.01) \\
(0.01)\end{array}$ & $\begin{array}{l}(0.06) \\
(0.10)\end{array}$ & $\begin{array}{l}(0.29) \\
(0.04)\end{array}$ & $\begin{array}{l}(0.08) \\
(0.03)\end{array}$ \\
\hline $\begin{array}{l}\mathrm{Cl} \\
\text { L.O.I. (Loss) }\end{array}$ & $(0.007)$ & $(0.015)$ & $(0.002)$ & & & \\
\hline $\begin{array}{l}\text { on-Ignition) } \\
\text { Total }\end{array}$ & $\begin{array}{c}1.49 \\
(99.908)\end{array}$ & $\begin{array}{c}1.79 \\
(100.05)\end{array}$ & $\begin{array}{c}0.92 \\
(100.04)\end{array}$ & $\begin{array}{r}(0.16) \\
(100.28)\end{array}$ & $\begin{array}{r}(0.17) \\
(100.20)\end{array}$ & $\begin{array}{r}(0.21) \\
(100.60)\end{array}$ \\
\hline
\end{tabular}

Values in parentheses are not certified, but are given for information only.

\section{Portland Cement Clinkers}

These RM's are intended primarily for use in the determination of the abundance of major phases in cement clinkers, i.e., the percentages of alite $\left(C_{3} S\right)^{*}$, belite $\left(C_{3} S\right)^{*}$, aluminate $\left(C_{3} A\right)^{*}$, and ferrite $\left(\left(C_{2}(A, F)\right)^{*}\right.$.

[*Note: Cement chemist's notation: $\mathrm{C}=\mathrm{CaO}, \mathrm{S}=\mathrm{SiO}_{2}, \mathrm{~A}=\mathrm{Al}_{2} \mathrm{O}_{3}, \mathrm{~F}=\mathrm{Fe}_{2} \mathrm{O}_{3}$.]

\begin{tabular}{lrrrr}
\hline \multicolumn{1}{c}{$\mathbf{R M}$} & & \multicolumn{3}{c}{ Concentration (wt. \%) } \\
\cline { 1 - 1 } Phase & $\mathbf{8 4 8 6}$ & $\mathbf{8 4 8 7}$ & $\mathbf{8 4 8 8}$ \\
\hline Alite & & $(58.47)$ & $(73.39)$ & $(64.97)$ \\
Belite & $(23.18)$ & $(7.75)$ & $(18.51)$ \\
Aluminate & $(1.15)$ & $(12.09)$ & $(4.34)$ \\
Ferrite & $(0.18)$ & $(3.27)$ & $(12.12)$ \\
Free CaO & $(3.21)$ & $(2.45)$ & $(0.00)$ \\
Periclase & $(0.14)$ & $(0.98)$ & $(0.05)$ \\
Alkali Sulfate & $(100.01)$ & $(100.02)$ & $(100.02)$ \\
Total & & & \\
\hline
\end{tabular}

Values in parentheses are not certified, but are given for information only. 


\section{Trace Elements}

The SRM's are for trace chemical analysis, specifically for calibrating instruments and evaluating analytical techniques used to determine trace elements in inorganic matrices.

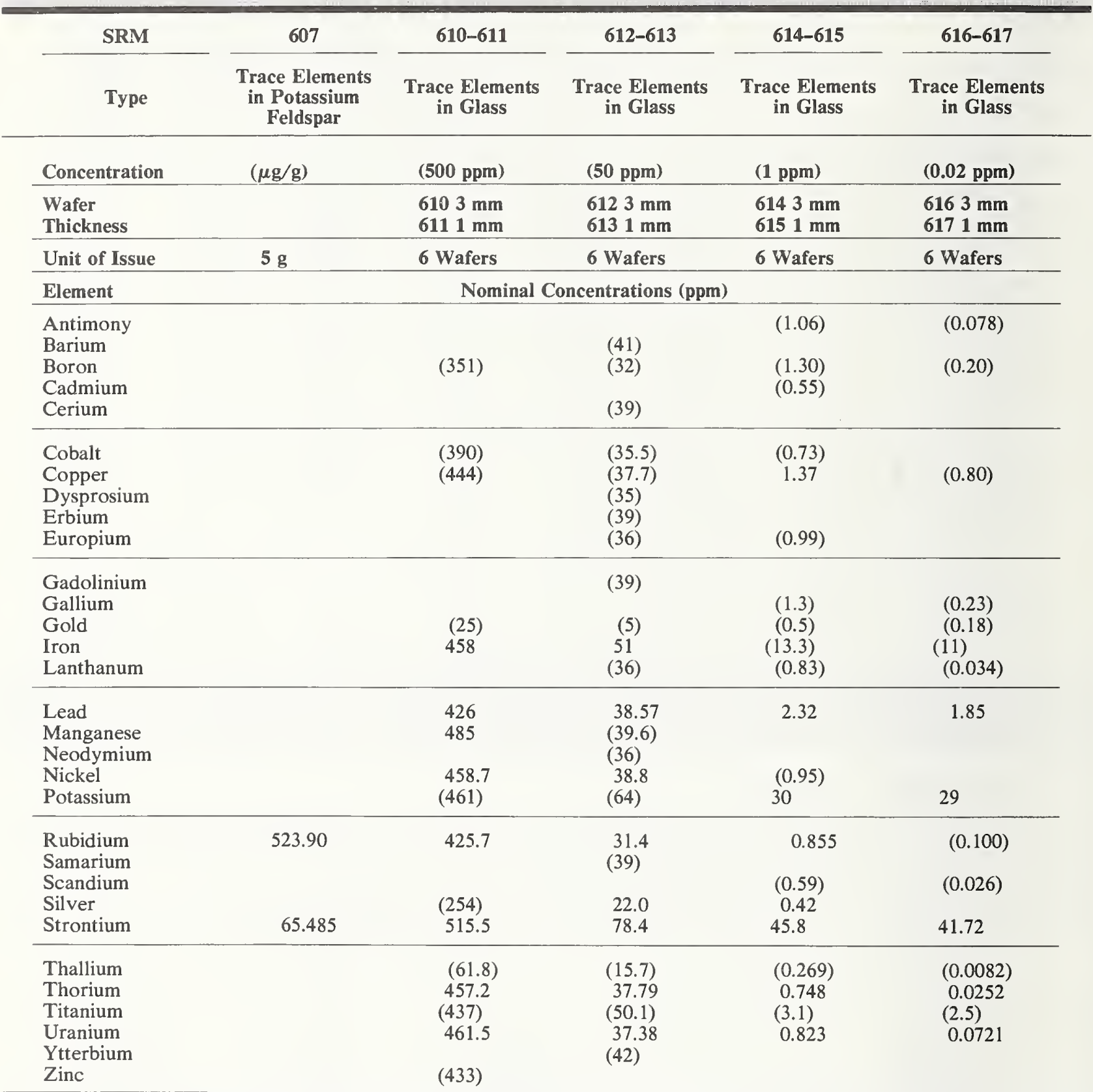

In addition to the elements listed above, the glass SRM's contain the following 25 elements: $\mathrm{As}, \mathrm{Be}, \mathrm{Bi}, \mathrm{Cs}, \mathrm{Cl}, \mathrm{F}, \mathrm{Ge}$, $\mathrm{Hf}, \mathrm{Hg}, \mathrm{Li}, \mathrm{Lu}, \mathrm{Mg}, \mathrm{Nb}, \mathrm{P}, \mathrm{Pr}, \mathrm{Se}, \mathrm{S}, \mathrm{Te}, \mathrm{Tb}, \mathrm{Tm}, \mathrm{Sn}, \mathrm{W}, \mathrm{V}, \mathrm{Y}$, and $\mathrm{Zr}$.

NOTE: Glass-Nominal Composition: $72 \% \mathrm{SiO}_{2}, 12 \% \mathrm{CaO}, 14 \% \mathrm{Na}_{2} \mathrm{O}$, and $2 \% \mathrm{Al}_{2} \mathrm{O}_{3}$.

Values in parentheses are not certified, but are given for information only. 


\section{Nuclear Radiation Monitoring}

\section{Radiation Dosimetry}

This SRM is a cobalt-in-aluminum alloy wire $0.5 \mathrm{~mm}$ in diameter and 1 meter long for use as a neutron density monitor standard.

\begin{tabular}{ccc}
\hline SRM & Identification (Batch Name) & $\begin{array}{c}\text { Cobalt Content (Weight } \\
\text { Percent) }\end{array}$ \\
\hline 953 & Neutron density monitor wire (Co in A1) & 0.116 \\
\hline
\end{tabular}

\section{Fission Track Glass}

This SRM contains uranium at three concentration levels, and will aid laboratories performing fission track analyses in interlaboratory comparisons of data and in monitoring neutron fluences. The material was irradiated in the NIST 10 Megawatt Research Reactor, at two different neutron energies.

The SRM unit contains four unirradiated glass wafers and two irradiated wafers.

\begin{tabular}{|c|c|c|c|c|c|}
\hline SRM & $\begin{array}{l}\text { Uranium Content } \\
\text { (ng/g) }\end{array}$ & U (Atom Percent) & $\begin{array}{l}\text { Reactor } \\
\text { Position }\end{array}$ & Cu Foil & Au Foil \\
\hline \multirow{3}{*}{$963 a$} & \multirow{3}{*}{0.823} & \multirow{3}{*}{0.2792} & & \multicolumn{2}{|c|}{ Neutron Fluence $\left(\times 10^{14} \mathrm{n} \cdot \mathrm{cm}^{-2}\right.$} \\
\hline & & & RT-3: & 41.2 & 45.8 \\
\hline & & & RT-4: & 39.5 & 43.0 \\
\hline
\end{tabular}




\section{Stable Isotopic Materials}

The isotopic composition of these SRM's has been determined by mass spectrometry.

\begin{tabular}{llll}
\hline \multicolumn{1}{c}{ Isotopic Reference Standards } & & \\
\hline SRM & \multicolumn{1}{c}{ Element Certified } & Wt/Unit (grams) \\
\hline 951 & Boric Acid & & \\
952 & Boric Acid, $95 \%$ Enriched ${ }^{10} \mathrm{~B}$ & Boron & 100 \\
975 & Sodium Chloride & Boron & 0.25 \\
976 & Copper Metal & Chlorine & 0.25 \\
977 & Sodium Bromide & Copper & 0.25 \\
\hline $978 \mathrm{a}$ & Silver Nitrate & Bromine & 0.25 \\
979 & Chromium Nitrate & Silver & 0.25 \\
980 & Magnesium Metal & Chromium & 0.25 \\
$* 981$ & Lead Metal, Natural & Magnesium & 0.25 \\
$* 982$ & Lead Metal, Equal Atom (206/208) & Lead & 1.0 \\
\hline$* 983$ & Lead Metal, Radiogenic (92\%-206) & Lead & 1.0 \\
984 & Rubidium Chloride, assay and isotopic & Lead & 1.0 \\
985 & Potassium Chloride, assay and isotopic & Rubidium & 0.25 \\
986 & Nickel & Potassium & 1.0 \\
987 & Strontium Carbonate, assay and isotopic & Nickel & 1.0 \\
\hline 989 & Rhenium, assay and isotopic & Strontium & 1.0 \\
990 & Silicon, assay and isotopic & Rhenium & pkg. (50) \\
991 & Lead-206 Spike, assay and isotopic & Silicon & wafer, 3 cm $\times 0.2 \mathrm{~cm}$ \\
994 & Gallium Metal, isotopic & Lead & 15 \\
997 & Thallium Metal, isotopic & Gallium & 0.25 \\
\hline
\end{tabular}

*Sold as a set containing SRM 981, 982, and 983.

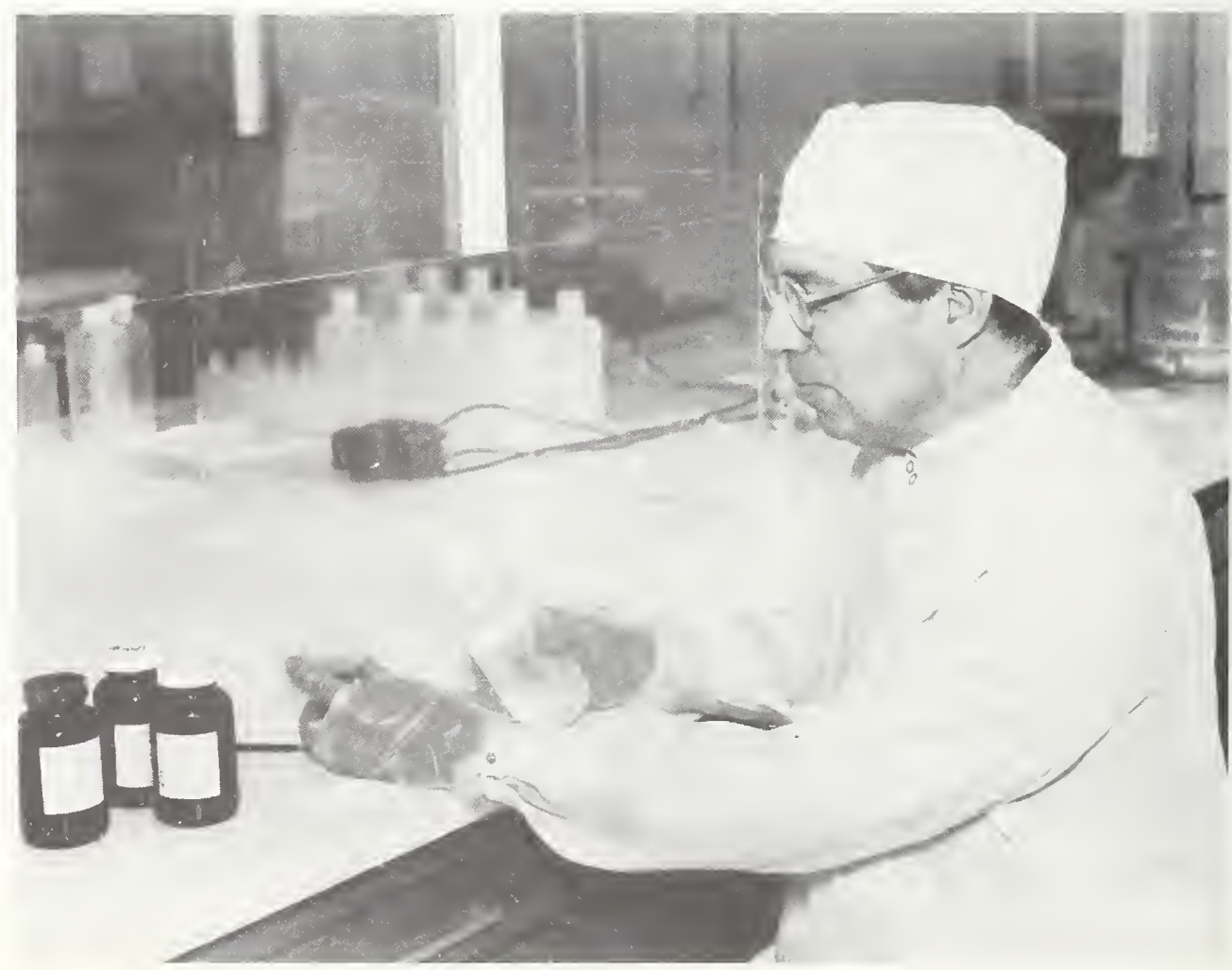

John Moody, a research chemist in the Inorganic Analytical Research Division, prepares some botanical materials in the clean room for trace analysis. 


\section{Physical Properties}

\section{ION ACTIVITY}

pH

These SRM's are used to prepare solutions of known hydrogen ion concentration to calibrate commercial pH instruments. SRM's 186Id and 186IId, 191a and 192a, and 922 and 923 are certified for use as admixtures only. SRM's 186Id and 186IId may be used to prepare a solution with a pH of 6.863 at $25^{\circ} \mathrm{C}$, or a physiological buffer solution with a $\mathrm{pH}$ of 7.41 at $25^{\circ} \mathrm{C}$.

\begin{tabular}{|c|c|c|c|}
\hline SRM & Type & $\begin{array}{l}\mathrm{pH}(\mathrm{S}) \text { Values } \\
\left(\text { at } 25^{\circ} \mathrm{C}\right)\end{array}$ & $\begin{array}{l}\text { Wt/Unit } \\
\text { (grams) }\end{array}$ \\
\hline $185 \mathrm{f}$ & Potassium hydrogen phthalate & 4.006 & 60 \\
\hline $\begin{array}{l}186 \mathrm{Id} \\
186 \mathrm{IId}\end{array}$ & $\begin{array}{l}\text { Potassium dihydrogen phosphate } \\
\text { Disodium hydrogen phosphate }\end{array}$ & (see above) & $\begin{array}{l}30 \\
30\end{array}$ \\
\hline $187 \mathrm{c}$ & Sodium tetraborate decahydrate (Borax) & 9.180 & 30 \\
\hline 188 & Potassium hydrogen tartrate & 3.557 & 60 \\
\hline $189 a$ & Potassium tetroxalate & 1.681 & 65 \\
\hline $\begin{array}{l}191 \mathrm{a} \\
192 \mathrm{a} \\
\end{array}$ & $\begin{array}{l}\text { Sodium bicarbonate } \\
\text { Sodium carbonate }\end{array}$ & 10.011 & $\begin{array}{l}25 \\
30\end{array}$ \\
\hline $\begin{array}{l}922 \\
923\end{array}$ & $\begin{array}{l}\text { Tris(hydroxymethyl)aminomethane } \\
\text { Tris(hydroxymethyl)aminomethane hydrochloride }\end{array}$ & 7.699 & $\begin{array}{l}25 \\
35\end{array}$ \\
\hline
\end{tabular}

\section{$p D$}

These SRM's are for the preparation of solutions of known deuterium-ion concentration to calibrate $\mathrm{pH}$ indicating equipment to indicate pD data. SRM's $2186 \mathrm{I}$ and 2186II, and 2191a and 2192a are certified for use as admixtures only.

\begin{tabular}{|c|c|c|c|}
\hline SRM & Type & $\mathrm{pD}(\mathrm{S})$ Values (at $25^{\circ} \mathrm{C}$ ) & $\begin{array}{l}\text { Wt/Unit } \\
\text { (grams) }\end{array}$ \\
\hline $\begin{array}{l}2181 \\
2182\end{array}$ & $\begin{array}{l}\text { Hepes/pH Buffer } \\
\text { Hepes/pH Buffer }\end{array}$ & $\begin{array}{l}7.503 \\
7.516 \\
\end{array}$ & $\begin{array}{l}60 \\
60\end{array}$ \\
\hline 2185 & Potassium hydrogen phthalate & 4.518 & 60 \\
\hline $\begin{array}{l}2186 \mathrm{I} \\
2186 \mathrm{II}\end{array}$ & $\begin{array}{l}\text { Potassium dihydrogen phosphate } \\
\text { Disodium hydrogen phosphate }\end{array}$ & 7.428 & $\begin{array}{l}30 \\
30 \\
\end{array}$ \\
\hline $\begin{array}{l}2191 \mathrm{a} \\
2192 \mathrm{a}\end{array}$ & $\begin{array}{l}\text { Sodium bicarbonate } \\
\text { Sodium carbonate }\end{array}$ & 10.732 & $\begin{array}{l}30 \\
30\end{array}$ \\
\hline
\end{tabular}




\section{Ion-Selective Electrodes}

These SRM's are certified for the calibration of ion-selective electrodes and have conventional ionic activities based on the Stokes-Robinson hydration theory for ionic strengths greater than 0.1 mole per liter.

\begin{tabular}{rlcc}
\hline SRM & Type & Certified Property & $\begin{array}{c}\text { Wt/Unit } \\
\text { (grams) }\end{array}$ \\
\hline & Sodium Chloride & $\mathrm{pNa}, \mathrm{pCl}$ & 125 \\
2201 & Potassium Chloride & $\mathrm{pK}, \mathrm{pCl}$ & 160 \\
2202 & Potassium Fluoride & $\mathrm{pF}$ & 125 \\
\hline
\end{tabular}

\section{METROLOGY}

\section{Scanning Electron Microscope (SEM)}

These SRM's are for calibrating the magnification scale and evaluating the performance of Scanning Electron Microscopes. SRM 484f has spacings of $0.5,1,2,5,10,30$, and $50 \mu \mathrm{m}$ and can be used to calibrate the magnification scale of an SEM from 1000 to $20,000 \mathrm{X}$ to an accuracy of 5 percent or better. SRM 2069a consists of graphitized natural fibers with smooth and uniform edges on an SEM specimen mount.

\begin{tabular}{|c|c|c|}
\hline SRM & Type & Size \\
\hline $484 \mathrm{f}$ & SEM Magnification Standard & $11 \mathrm{~mm} \mathrm{D}, 0.65 \mathrm{~mm}$ high \\
\hline $2069 a$ & SEM Performance Standard & $12 \mathrm{~mm} \mathrm{D}, 3 \mathrm{~mm}$ peg \\
\hline
\end{tabular}

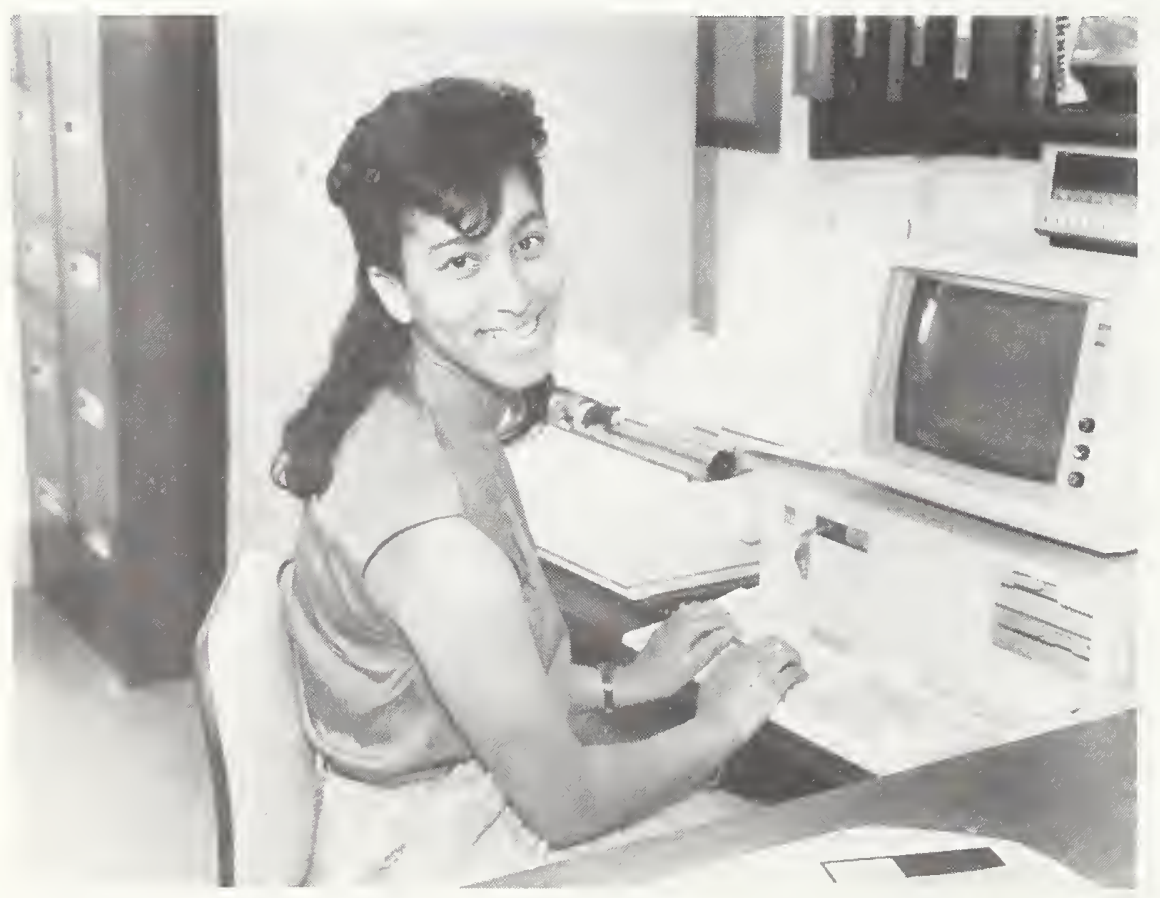

Robbin Frazer creates a warm, friendly atmosphere in the certification and production office where she helps perform the documentation and records keeping for SRM certification projects. 


\section{Optical Microscope Linewidth-Measurement}

These SRM's are for use in calibrating optical microscopes used to measure the widths of opaque lines and clear spaces on integrated-circuit photomasks. They can also be used to calibrate line spacings and line-tospace ratios. The accuracy of a measured linewidth or line spacing is $\pm 0.05 \mu \mathrm{m}$ or better. They are not for use with partially transmitting materials, in reflected light with opaque materials, or in a scanning electron microscope. SRM 475 is made with anti-reflective chromium on a borosilicate glass substrate. SRM 476 is made with bright chromium.

\begin{tabular}{|c|c|c|c|}
\hline SRM & Type & Spacings & Size \\
\hline 473 & Optical Linewidth Standard IN PREP & & \\
\hline 475 & Linewidth Measurement Standard & 0.5 to $12 \mu \mathrm{m}$ & $6.35 \times 6.35 \times 0.15 \mathrm{~cm}$ \\
\hline 476 & Linewidth Measurement Standard IN PREP & 0.5 to $12 \mu \mathrm{m}$ & $6.35 \times 6.35 \times 0.15 \mathrm{~cm}$ \\
\hline
\end{tabular}

\section{Depth Profiling}

This SRM is for calibrating equipment used to measure sputtered depth and erosion rates in surface analysis. SRM 2135c consists of nine alternating metal thin-film layers-five layers of pure chromium and four of pure nickel-on a polished silicon (100) substrate. It is certified for total chromium and total nickel thickness, for individual layer uniformity, for $\mathrm{Ni} / \mathrm{Cr}$ bi-layer uniformity, and for individual layer thickness. The nominal thicknesses for $\mathrm{Cr}$ and $\mathrm{Ni}$ are 53 and $66 \mathrm{~nm}$, respectively.

\begin{tabular}{|c|c|c|c|}
\hline SRM & Type & & Unit/Size \\
\hline $\begin{array}{l}2135 c \\
2136 \\
2137\end{array}$ & $\begin{array}{l}\text { Ni-Cr Thin-Film Depth Profile Standard } \\
\mathrm{Cr} / \mathrm{CrO} \text { Thin-Film Depth Profile Standard } \\
\text { Boron Implant in Silicon Depth Profile }\end{array}$ & $\begin{array}{l}\text { IN PREP } \\
\text { IN PREP }\end{array}$ & $\begin{array}{l}1 \times 2.54 \times 0.04 \mathrm{~cm} \\
1 \times 2.54 \times 0.04 \mathrm{~cm}\end{array}$ \\
\hline
\end{tabular}

Lorna Sniegoski, research chemist in the Organic Analytical Research Division, uses a microbalance to weigh an analyte to prepare a solution for analysis of an SRM.

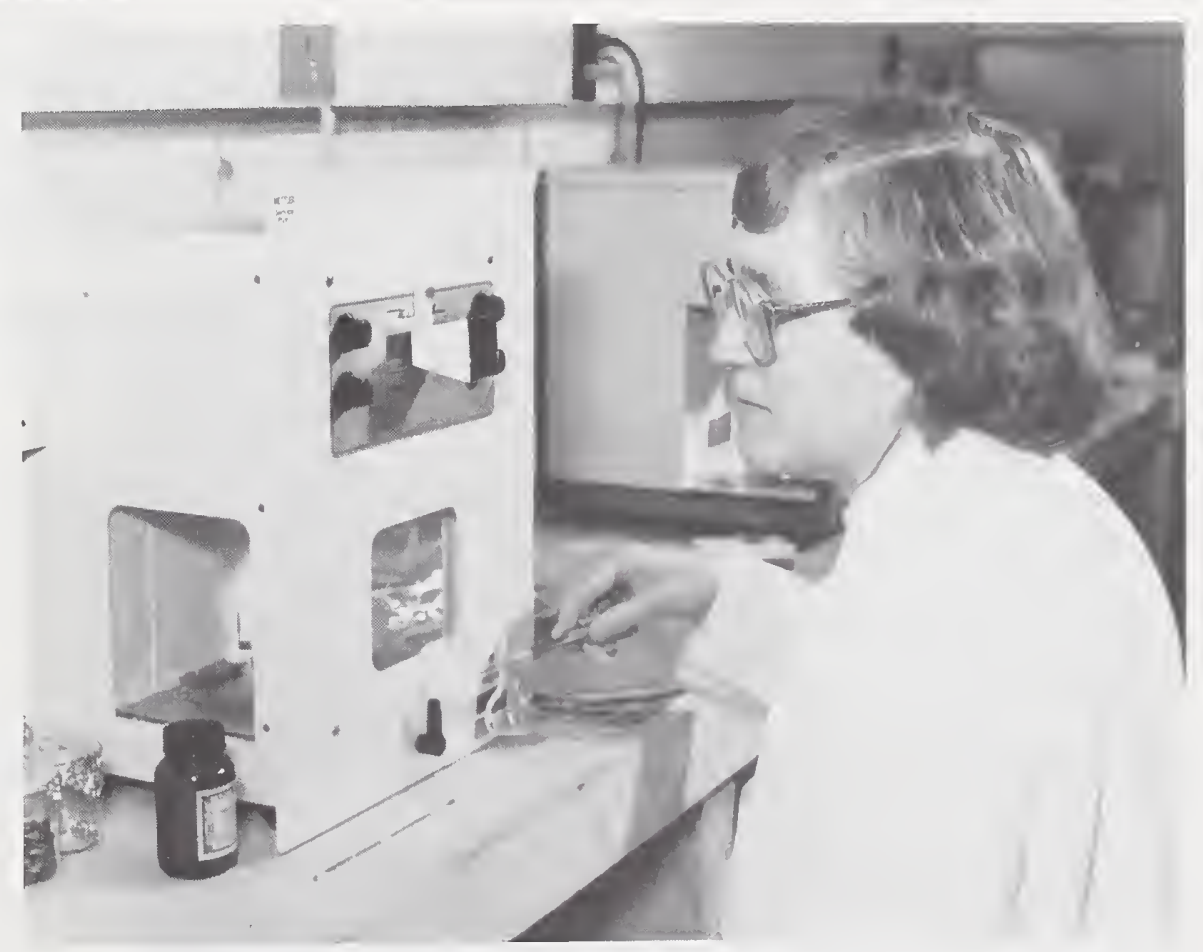




\section{COATING THICKNESS}

These magnetic type thickness SRM's are $30 \times 30 \mathrm{~mm}$ for calibrating coating thickness gages used to measure the thickness of nonmagnetic coatings on steel, or nickel on steel. The steel substrates have the properties of AISI 1010 steel and the nickel coatings have the properties of an annealed Watts nickel electrodeposited free of cobalt and iron.

These SRM's may be used to measure the thickness of paint and other organic coatings on steel, as well as zinc (galvanized) and other nonmagnetic metallic coatings.

\section{Nonmagnetic Coating on Magnetic Substrate (Cu and $\mathrm{Cr}$ on Steel)}

\begin{tabular}{|c|c|c|c|}
\hline \multirow{2}{*}{ SRM } & \multirow{2}{*}{ Unit Size } & \multicolumn{2}{|c|}{ Nominal Coating Thickness } \\
\hline & & micrometer & milliinch (mil) \\
\hline 1321 & Set of 4 & $34,37,42,47$ & $7.4,1.5,1.7,1.9$ \\
\hline 1322 & Set of 4 & $53,60,69,80$ & $2.1,2.4,2.8,3.2$ \\
\hline 1323 & Set of 4 & $4,112,135,167$ & $3.8,4.5,5.4,6.7$ \\
\hline 1357 & Set of 3 & $6,20,48$ & $0.24,0.8,1.9$ \\
\hline 1358 & Set of 3 & $80,225,1000$ & $3.1,10,39$ \\
\hline 1359 & Set of 4 & $48,140,505,800$ & $2.0,5.5,20,32$ \\
\hline 1360 & Set of 4 & $2.5,6,12,20$ & $0.1,0.2,0.5,0.8$ \\
\hline $1361 \mathrm{a}$ & Set of 4 & $6,12,25,48$ & $0.2,0.5,1.0,2.0$ \\
\hline $1362 \mathrm{a}$ & Set of 4 & $40,80,140,205$ & $1.6,3.1,5.5,7.9$ \\
\hline $1363 \mathrm{a}$ & Set of 4 & $255,385,505,635$ & $9.8,16,20,26$ \\
\hline $1364 a$ & Set of 4 & $800,1000,1525,1935$ & $32,39,59,79$ \\
\hline
\end{tabular}

\section{Magnetic Coating on Magnetic Substrate (Nickel on Steel)}

\begin{tabular}{cccc}
\hline \multirow{2}{*}{ SRM } & Unit Size & \multicolumn{2}{c}{ Nominal Coating Thickness } \\
\cline { 3 - 4 } & & micrometer & milliinch (mil) \\
\hline $1365 \mathrm{a}^{*}$ & Set of 4 & $3,9,15,20$ & $0.1,0.4,0.6,0.8$ \\
$1366 \mathrm{a}^{*}$ & Set of 4 & $25,34.5,40,50$ & $1.0,1.4,1.6,2.0$ \\
\hline
\end{tabular}

*SRM's 1365 a and 1366a will be reissued as SRM's 1331-1334 and 1335-1338, respectively.

\section{Solder Thickness Standards}

\begin{tabular}{|c|c|c|}
\hline SRM & Type & Unit Size \\
\hline 2321 & Solder Thickness & IN PREP \\
\hline 2322 & Solder Thickness & IN PREP \\
\hline
\end{tabular}




\section{COATING WEIGHT}

The gold coating SRM's are $15 \times 15 \mathrm{~mm}$ and were measured by beta-ray backscatter and $\mathrm{x}$-ray fluorescence techniques relative to NIST gold coating materials for which the average weights per unit area were determined by weight and area measurements. These SRM's are for calibrating equipment used to measure weight per unit area of gold coating of equivalent purity.

\section{Gold Coating on Nickel}

\begin{tabular}{|c|c|c|c|c|}
\hline \multirow{2}{*}{ SRM } & \multirow{2}{*}{ Unit Size } & \multirow{2}{*}{$\begin{array}{l}\text { Nominal Coating } \\
\text { Weight }\left(\mathrm{mg} / \mathrm{cm}^{2}\right)\end{array}$} & \multicolumn{2}{|c|}{ Nominal Coating Thickness } \\
\hline & & & micrometer & microinch \\
\hline 1379 & 1 each & 0.35 & 0.175 & 7 \\
\hline 1380 & 1 each & 0.55 & 0.275 & 11 \\
\hline 1387 & 1 each & 2.2 & 1.4 & 45 \\
\hline $1399 b$ & Set of 4 & $1.5,3.0,6.0,14.0$ & $0.8,1.5,3,7$ & $30,60,120,280$ \\
\hline
\end{tabular}

\section{ELLIPSOMETRY}

Each of these SRM's is certified for the ellipsometric parameters of delta $(\Delta)$ and psi $(\Psi)$ and the derived thickness and refractive index of the silicon dioxide layer on the silicon wafer.

\begin{tabular}{cccc}
\hline & & & \\
\hline SRM & \multicolumn{1}{c}{ Type } & Unit Size & Nominal Thickness \\
\hline & & & \\
2531 & Thin Film Thickness & IN PREP & $50 \mathrm{~nm}$ \\
2532 & Thin Film Thickness & 76 mm Dia wafer & $100 \mathrm{~nm}$ \\
2533 & Thin Film Thickness & IN PREP & $200 \mathrm{~nm}$ \\
2534 & Thin Film Thickness & IN PREP & $25 \mathrm{~nm}$ \\
\hline
\end{tabular}

Carmelita Davis exhibits a warm, expressive personality and dependability as she handles the Materials Safety Date Sheets along with other responsibilities.

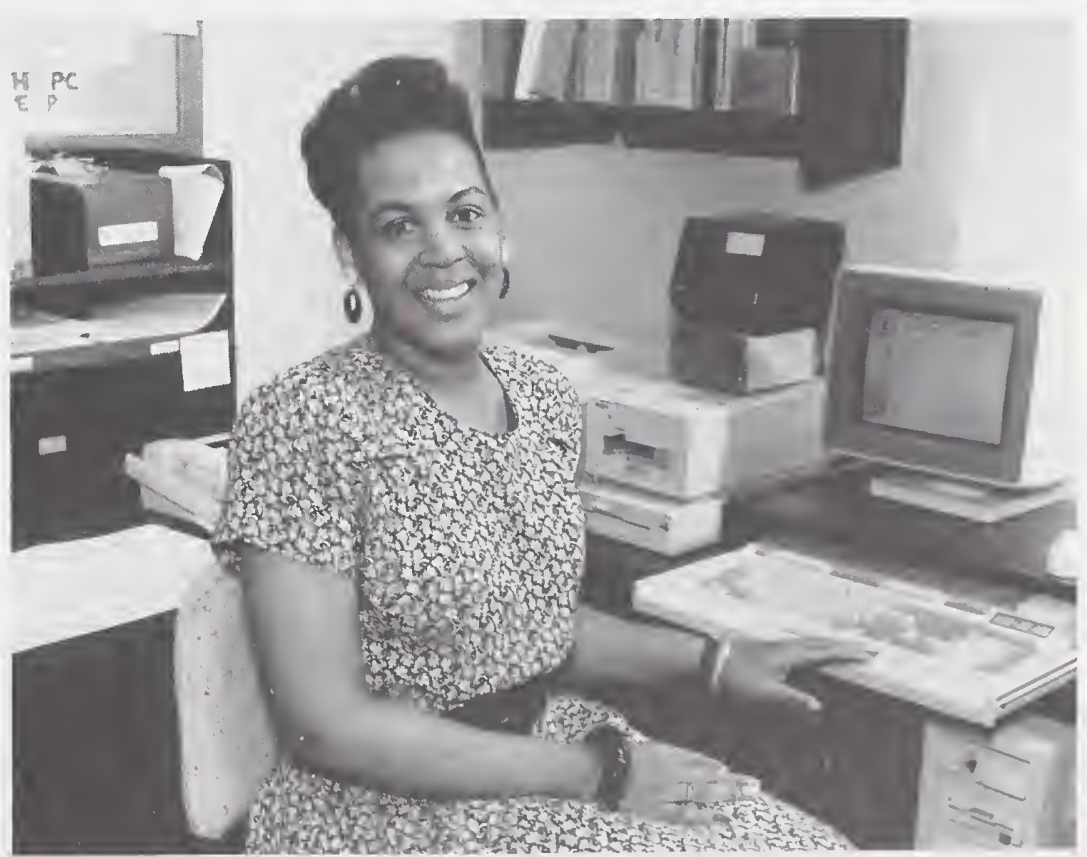




\section{Chemical Resistance (Durability) of Glass}

These SRM's are for checking test methods and calibrating equipment used to determine the resistance of glass containers to chemical attack. The values below represent the volume of fiftieth-normal sulfuric acid used to titrate to the methyl-red end point the alkaline extract from a crushed sample of glass after exposure to high-purity water at $121^{\circ} \mathrm{C}$.

\begin{tabular}{ccccc}
\hline & & & \\
\hline SRM & Type & Unit of Issue & mL of N/50 $\mathbf{H}_{\mathbf{2}} \mathrm{SO}_{\mathbf{4}}$ \\
\hline & & & \\
622 & Soda-lime-silica & $2.2 \mathrm{~kg}$ & 7.67 \\
623 & Borosilicate & $2.2 \mathrm{~kg}$ & 0.34 \\
\hline
\end{tabular}

\section{Electrical Properties of Glass}

SRM 624 is for checking test methods and for calibrating equipment used to determine the dc volume resistivity of glass per ASTM C657. SRM 774 is for checking methods used to determine dielectric constant and ac loss characteristics of insulating materials per ASTM D150.

\begin{tabular}{clll}
\hline & & & \\
\hline SRM & \multicolumn{1}{c}{ Type } & Unit of Issue & Approximate Value \\
\hline 624 & Lead-silica, for de resistivity & $200 \mathrm{~g}$ & $\log _{10} \rho \sim 9.9 \Omega-\mathrm{cm}$ \\
774 & Lead-silica, for dielectric constant & $5 \times 5 \times 2.5 \mathrm{~cm}$ & $\mathrm{~K} \sim 7.47$ \\
\hline
\end{tabular}

\section{Viscosity}

SRM's 710a, 711, and 717 are rectangular bars for checking the performance of high-temperature viscosity equipment (rotating cylinders) and low-temperature viscosity equipment (fiber elongation, beam-bending, parallel-plates, etc.).

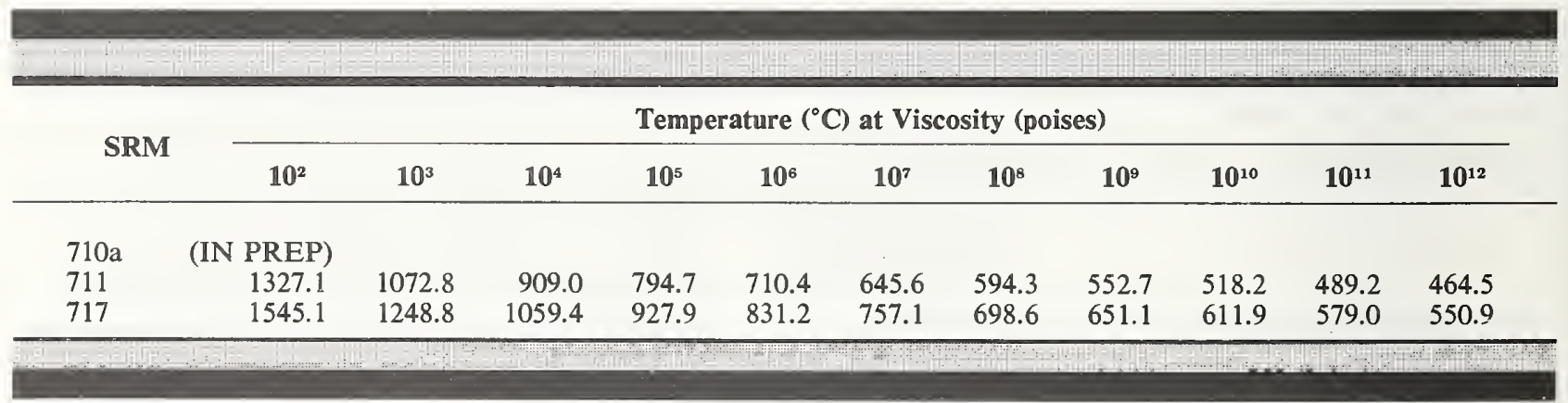




\section{Viscosity Fixpoints}

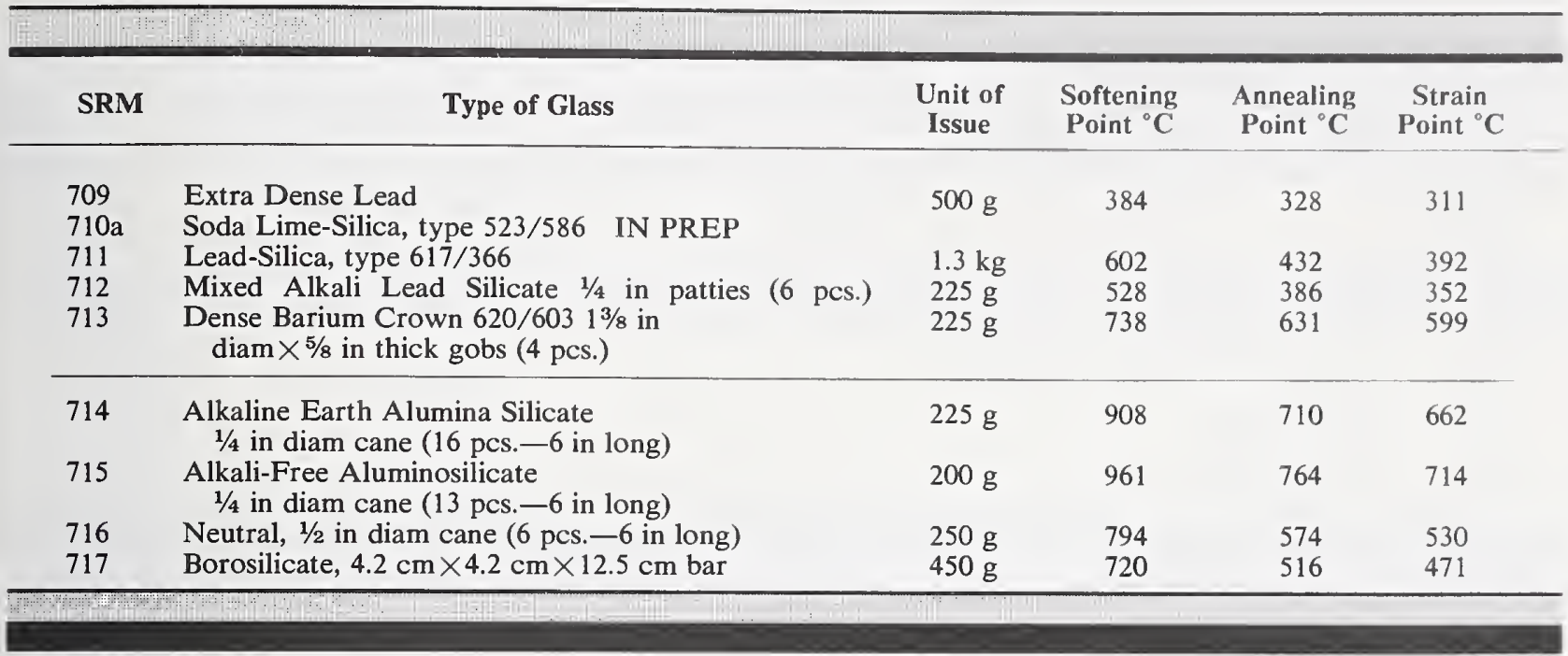

\section{Relative Stress Optical Coefficient}

These glasses are for calibrating instruments used to measure relative stress optical coefficient per ASTM C770. They are rectangular bars.

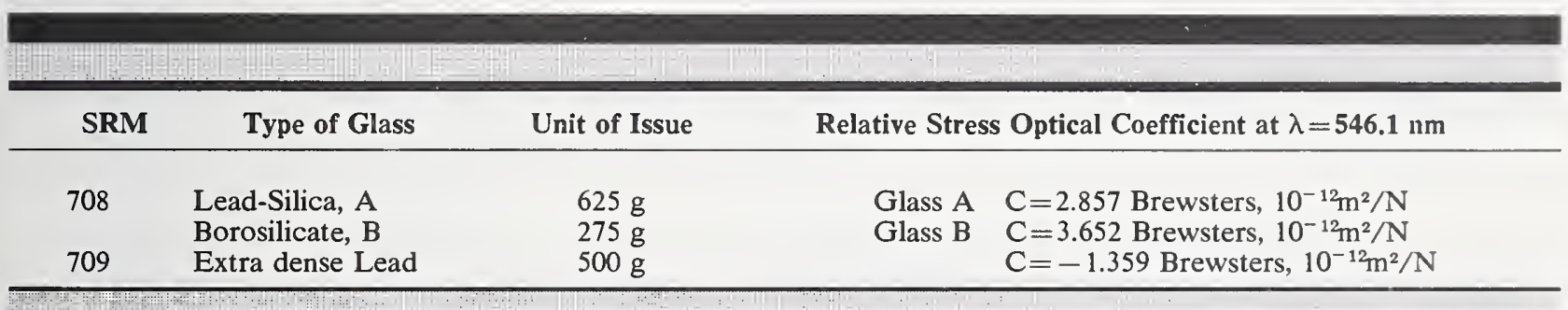

-

\section{Glass Liquidus Temperature}

This SRM is for checking test methods and for calibrating equipment used to determine the liquidus temperature of glass by the gradient furnace methods per ASTM C829.

\begin{tabular}{cccc}
\hline SRM & Type & Unit of Issue & Temperature, ${ }^{\circ} \mathrm{C}$ \\
\hline 773 & $\begin{array}{c}\text { Soda-lime-silica, for liquidus temperature } \\
2.5 \times 2.5 \times 0.6 \mathrm{~cm}\end{array}$ & $60 \mathrm{~g}$ & 990 \\
\hline
\end{tabular}




\section{Density}

SRM's 211c, 2211, and 2213 are certified for density (air saturated at $1 \mathrm{~atm}$ ) at 20,25 , and $30{ }^{\circ} \mathrm{C}$, and may be used to calibrate pycnometers and density balances.

SRM's 1840 and $1841 \mathrm{a}$ are certified for density at $20^{\circ} \mathrm{C}$ and may be used to determine the density of solids and liquids by means of hydrostatic weighing.

\begin{tabular}{|c|c|c|c|}
\hline SRM & Type & Density $20^{\circ} \mathrm{C}\left(\mathrm{g} / \mathrm{cm}^{3}\right)$ & Amount \\
\hline $211 \mathrm{c}$ & Toluene & 0.86686 & $5 \mathrm{~mL}$ \\
\hline 2211 & Toluene & 0.86686 & $8 \mathrm{~mL}$ \\
\hline 2213 & $2,2,4$ Trimethylpentane (Isooctane) & 0.691929 & $25 \mathrm{~mL}$ \\
\hline $1841 \mathrm{a}$ & Silicon & 2.329 & $200 \mathrm{~g}$ \\
\hline 1825 & Glass Density & 2.20185 & $22 \mathrm{~g}$ \\
\hline 1826 & Glass Density & 2.54938 & $21 \mathrm{~g}$ \\
\hline 1827 & Glass Density & 3.0495 & $20 \mathrm{~g}$ \\
\hline
\end{tabular}

\section{Microhardness}

These SRM's are for use in calibrating and checking the performance of microhardness testers. These test blocks were made by electroforming the test metal on a steel substrate. The hardness numbers for 1893 through 1896 are each certified at loads of 25, 50, and 100-gram force, while 1905, 1906, and 1907 are certified for 300,500 , and 1000 gram-force, respectively.

\begin{tabular}{clllll}
\hline & & & & \\
\hline & & & Load & Hardness & \\
\hline & Type & & & \\
1893 & Bright Copper (Knoop) & $25,50,100 \mathrm{~g}-\mathrm{f}$ & $125 \mathrm{~kg} / \mathrm{mm}^{2}$ & $12.5 \mathrm{~mm} \mathrm{square}$ \\
1894 & Bright Copper (Vickers) & $25,50,100 \mathrm{~g}-\mathrm{f}$ & $125 \mathrm{~kg} / \mathrm{mm}^{2}$ & $12.5 \mathrm{~mm} \mathrm{square}$ \\
1895 & Bright Nickel (Knoop) & $25,50,100 \mathrm{~g}-\mathrm{f}$ & $600 \mathrm{~kg} / \mathrm{mm}^{2}$ & $12.5 \mathrm{~mm} \mathrm{square}$ \\
1896 & Bright Nickel (Vickers) & $25,50,100 \mathrm{~g}-\mathrm{f}$ & $600 \mathrm{~kg} / \mathrm{mm}^{2}$ & $12.5 \mathrm{~mm} \mathrm{square}$ \\
1905 & Bright Nickel (Knoop) & $300 \mathrm{~g}-\mathrm{f}$ & $600 \mathrm{~kg} / \mathrm{mm}^{2}$ & $12.5 \mathrm{~mm} \mathrm{square}$ \\
1906 & Bright Nickel (Knoop) & $500 \mathrm{~g}-\mathrm{f}$ & $600 \mathrm{~kg} / \mathrm{mm}^{2}$ & $12.5 \mathrm{~mm} \mathrm{square}$ \\
1907 & Bright Nickel (Knoop) & $1000 \mathrm{~g}-\mathrm{f}$ & $600 \mathrm{~kg} / \mathrm{mm}^{2}$ & $12.5 \mathrm{~mm} \mathrm{square}$ \\
\hline
\end{tabular}

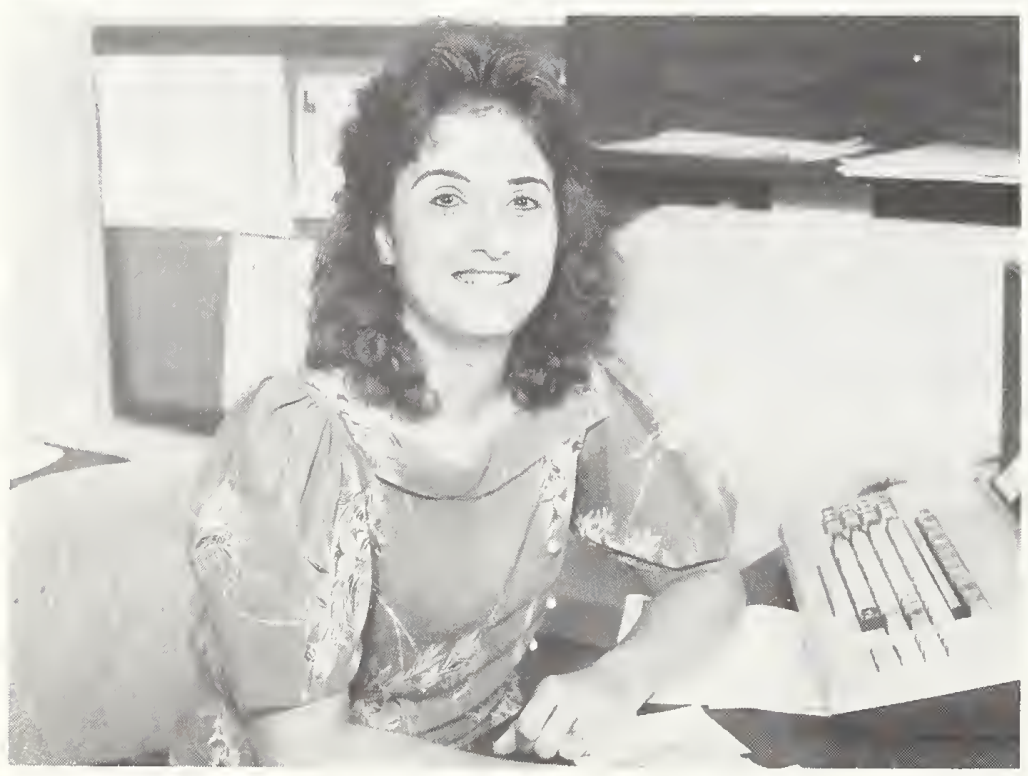

Gina Montgomery, a friendly, outgoing member of the customer sales and service staff, maintains and updates the SRM customer mail lists with valued dependability. 


\section{Ultrasonics}

SRM 1855 is for point-by-point calibration of apparatus used to measure ultrasonic power. SRM 1856 is a displacement-measuring transducer to be used to determine the size and character of surf ith 'ibrations.

\begin{tabular}{|clll|}
\hline & Type & Frequency Range & Unit \\
\hline SRM & & & \\
1855 & Ultrasonic Power Transducer & 1.6 to $21.6 \mathrm{MHz}$ & Each \\
1856 & Acoustic Emission Transducer & 0.1 to $1 \mathrm{MHz}$ & Each \\
\hline
\end{tabular}

\section{Polymers}

wing

\section{Molecular Weight (Melt Flow)}

\begin{tabular}{|c|c|c|}
\hline SRM & Type & $\begin{array}{l}\text { Wt/Unit } \\
\text { (grams) }\end{array}$ \\
\hline $\begin{array}{r}705 \\
706 \\
1474 \\
1475 \\
1476\end{array}$ & $\begin{array}{l}\text { Polystyrene, narrow molecular weight distribution, } \mathbf{M}_{\mathrm{w}} \approx 179,300, \mathrm{M}_{\mathrm{w}} / \mathrm{M}_{\mathrm{n}} \approx 1.07 \\
\text { Polystyrene, broad molecular weight distribution, } \mathrm{M}_{\mathrm{w}} \approx 257,800, \mathrm{M}_{\mathrm{w}} / \mathrm{M}_{\mathrm{n}} \approx 2.1 \\
\text { Polyethylene Melt Flow } \\
\text { Polyethylene, linear, } \mathrm{M}_{\mathrm{w}} \approx 52,000, \mathrm{M}_{\mathrm{w}} / \mathrm{M}_{\mathrm{n}} \approx 2.9 \\
\text { Polyethylene, branched }\end{array}$ & $\begin{array}{l}5 \\
18 \\
\text { IN PREP } \\
50 \\
50\end{array}$ \\
\hline $\begin{array}{l}1478 \\
1479 \\
1480 \\
1481 \\
1482\end{array}$ & $\begin{array}{l}\text { Polystyrene, narrow molecular weight distribution, } M_{\mathrm{w}} \approx 37,400\left(\mathrm{M}_{\mathrm{w}} / \mathrm{M}_{\mathrm{n}} \approx 1.04\right) \\
\text { Polystyrene, narrow molecular weight distribution, } \mathrm{M}_{\mathrm{w}} \approx 1,050,000 \\
\text { Polyurethanes Low } \mathrm{MW} \\
\text { Polyurethanes HI } \mathrm{MW} \\
\text { Polyethylene, linear, } \mathrm{M}_{\mathrm{w}} \approx 13,600\left(\mathrm{M}_{\mathrm{w}} / \mathrm{M}_{\mathrm{n}} \approx 1.19\right)\end{array}$ & $\begin{array}{l}2 \\
2 \\
\text { IN PREP } \\
\text { IN PREP } \\
1\end{array}$ \\
\hline $\begin{array}{l}1483 \\
1484 \\
1487 \\
1488 \\
1489\end{array}$ & $\begin{array}{l}\text { Polyethylene, linear, } M_{w} \approx 32,100\left(M_{w} / M_{n} \approx 1.11\right) \\
\text { Polyethylene, linear, } M_{w} \approx 119,600\left(M_{w} / M_{n} \approx 1.19\right) \\
\text { Poly(methylmethacrylate), } M_{n} \approx 6,000 \\
\text { Poly(methylmethacrylate), } M_{n} \approx 29,000 \\
\text { Poly(methylmethacrylate), } M_{n} \approx 115,000\end{array}$ & $\begin{array}{l}1 \\
1 \\
2 \\
2 \\
2\end{array}$ \\
\hline $\begin{array}{l}1496 \\
1497 \\
1923 \\
1924 \\
1925\end{array}$ & $\begin{array}{l}\text { Polyethylene Gas Pipe Resin (Unpigmented) } \\
\text { Polyethylene Gas Pipe Resin (Pigmented) } \\
\text { Polystyrene Sulfonates } \\
\text { Polystyrene Sulfonates } \\
\text { Polystyrene Sulfonates }\end{array}$ & $\begin{array}{l}2 \mathrm{lbs} . \\
20 \mathrm{lbs} . \\
\text { IN PREP } \\
\text { IN PREP } \\
\text { IN PREP }\end{array}$ \\
\hline $\begin{array}{l}8450 \\
8451 \\
8452 \\
8453 \\
8454\end{array}$ & $\begin{array}{l}\text { Polyethylene Piping } 1 / 2 \text { in } \\
\text { Polyethylene Piping } 2 \text { in } \\
\text { Polyethylene Piping } 4 \text { in } \\
\text { Polyethylene Piping Socket T } \\
\text { Polyethylene Piping Butt T }\end{array}$ & $\begin{array}{l}2 \text { Pieces } \\
2 \text { Pieces } \\
2 \text { Pieces } \\
3 \text { Pieces } \\
3 \text { Pieces }\end{array}$ \\
\hline
\end{tabular}


These materials are certified for the properties indicated in the table.

\begin{tabular}{|c|c|c|c|c|c|c|c|c|c|c|c|c|c|}
\hline Property & Method & 705 & 706 & 475 & 1476 & 1478 & 1479 & 91482 & 1483 & 1484 & 41487 & 71488 & 1489 \\
\hline $\begin{array}{l}\text { Molecular Weight: } \\
\text { Weight Average }\end{array}$ & $\begin{array}{l}\text { (Light Scattering) } \\
\text { (Sed. Equili.) } \\
\text { (Gel Permeation } \\
\text { Chromatography- } \\
\text { GPC) }\end{array}$ & $\begin{array}{l}X \\
X\end{array}$ & $\begin{array}{l}X \\
X\end{array}$ & $\begin{array}{l}X \\
X\end{array}$ & & $\mathrm{X}$ & $\mathrm{X}$ & $X$ & $\mathrm{X}$ & $\mathrm{X}$ & $\mathrm{X}$ & $X$ & \\
\hline Number Average & $\begin{array}{l}\text { (Osmometry) } \\
\text { (GPC) }\end{array}$ & $\mathrm{X}$ & & $\mathrm{X}$ & & $\mathrm{X}$ & & $\mathrm{X}$ & $\mathrm{X}$ & $\mathrm{X}$ & & & $\mathrm{X}$ \\
\hline $\begin{array}{l}\text { Molecular Weight } \\
\text { Distribution }\end{array}$ & (GPC) & & & $\mathrm{X}$ & & & & & & & & & \\
\hline $\begin{array}{l}\text { Limiting Viscosity No. } \\
\text { Benzene } 25^{\circ} \mathrm{C} \\
\text { Benzene } 35^{\circ} \mathrm{C} \\
\text { Cyclohexane } 35^{\circ} \mathrm{C} \\
\text { 1-Chloronaphthalene } \\
\text { 1,2,4-trichlorobenzene } \\
\text { Decahydronaphthalen } \\
\text { Tetrahydrofuran } 25^{\circ} \mathrm{C} \\
\text { Toluene } 25^{\circ} \mathrm{C}\end{array}$ & $\begin{array}{l}\text { (Capillary Viscometer) } \\
130^{\circ} \mathrm{C} \\
130^{\circ} \mathrm{C} \\
\text { C } 130^{\circ} \mathrm{C}\end{array}$ & $\begin{array}{l}X \\
X \\
X\end{array}$ & $\begin{array}{l}X \\
X\end{array}$ & $\begin{array}{l}X \\
X \\
X\end{array}$ & $\begin{array}{l}X \\
X \\
X\end{array}$ & $\mathrm{X}$ & & $\begin{array}{l}X \\
X\end{array}$ & $\begin{array}{l}X \\
X\end{array}$ & $\begin{array}{l}X \\
X\end{array}$ & $\mathrm{X}$ & $X$ & $\mathrm{X}$ \\
\hline Melt Flow & (ASTM) & & & $\mathrm{X}$ & $\mathrm{X}$ & & & & & & & & \\
\hline Density & (ASTM) & & & $\mathrm{X}$ & $\mathrm{X}$ & & & & & & & & \\
\hline Heat Capacity & (Adiabatic) & $\mathrm{X}$ & & $\mathrm{X}$ & & & & & & & & & \\
\hline
\end{tabular}

\section{Rheology}

This SRM is for calibrating instruments used in polymer technology and science to determine rheological properties of polymer melts or solutions. It is certified for Rate of Shear, Viscosity, and First Normal Stress Difference at $25^{\circ} \mathrm{C}$.

\begin{tabular}{|ccc}
\hline & & \\
\hline SRM & Type & Unit size \\
\hline $1490 \quad$ Polyisobutylene Solution in Cetane & $250 \mathrm{~mL}$ \\
\hline
\end{tabular}




\section{Heat}

\section{Calorimetric}

These SRM's are intended to relate the gain or loss of energy and work experienced during a chemical reaction or by change of temperature to the units of energy and work as defined by the International System of Units (SI). The unit for energy and work under this system is the joule, which is related to the calorie by the equation: 4.184 joule $=1$ calorie.

\begin{tabular}{|c|c|c|c|}
\hline SRM & Type & $\begin{array}{l}\text { Approximate Heat of } \\
\text { Combustion }(\mathrm{MJ} / \mathrm{kg})\end{array}$ & Unit Amount \\
\hline $\begin{array}{r}39 \mathrm{i} \\
2213 \\
1656 \\
1657\end{array}$ & $\begin{array}{l}\text { Benzoic Acid } \\
2,2,4-T r i m e t h y l p e n t a n e ~(I s o o c t a n e) \\
\text { Thianthrene } \\
\text { Synthetic Refuse Derived Fuel }\end{array}$ & $\begin{array}{l}26.434 \\
47.712 \\
33.480 \\
13.87\end{array}$ & $\begin{array}{l}30 \mathrm{~g} \\
25 \mathrm{~mL} \\
30 \mathrm{~g} \\
100 \mathrm{~g}\end{array}$ \\
\hline $\begin{array}{l}2151 \\
2152 \\
2683 a \\
2684 a \\
2685\end{array}$ & $\begin{array}{l}\text { Nicotinic Acid } \\
\text { Urea } \\
\text { Coal, Bituminous: } \% \mathrm{~S}=1.85 ; \% \mathrm{Ash}=6.85 \\
\text { Coal, Bituminous: } \% \mathrm{~S}=3.00 ; \% \mathrm{Ash}=11.09 \\
\text { Coal, Bituminous: } \% \mathrm{~S}=4.62 ; \% \mathrm{Ash}=16.53\end{array}$ & $\begin{array}{l}22.184 \\
10.536 \\
32.30(13887 \mathrm{Btu} / \mathrm{lb}) \\
28.92(12433 \mathrm{Btu} / \mathrm{lb}) \\
27.38(11770 \mathrm{Btu} / \mathrm{lb})\end{array}$ & $\begin{array}{l}25 \mathrm{~g} \\
25 \mathrm{~g} \\
50 \mathrm{~g} \\
50 \mathrm{~g} \\
50 \mathrm{~g}\end{array}$ \\
\hline
\end{tabular}

NOTE: The calorific values $(\mathrm{MJ} / \mathrm{kg}$ ) may decrease upon the aging or normal oxidation of the coals. NIST will continue to monitor these calorific values and report any substantive change to the purchaser.

\section{Solution Calorimetric}

\begin{tabular}{cccc}
\hline SRM & Type & $\begin{array}{c}\text { Heat of Solution } \\
(\mathbf{M J} / \mathrm{kg})\end{array}$ & $\begin{array}{c}\text { Wt/Unit } \\
(\mathrm{grams})\end{array}$ \\
\hline $724 \mathrm{a}$ & $\begin{array}{l}\text { Tris(hydroxymethyl)aminomethane } \\
\text { (Hydrochloric Acid and Sodium Hydroxide Solution Calori- } \\
\text { metry) }\end{array}$ & $\begin{array}{c}\text { Evolved } 0.24576 \\
\text { Absorbed } 0.1418\end{array}$ \\
\hline 1655 & Potassium Chloride (Water Solution Calorimetry) & Absorbed (0.235) \\
\hline
\end{tabular}

\section{Heat Source Calorimetric}

SRM

Type
Heat of Evolution (MJ/kg)

1.46

1.632
Wt/Unit (grams)
1651 Zirconium-barium chromate heat source powder

1652 Zirconium-barium chromate heat source powder

1653 Zirconium-barium chromate heat source powder
50

50

50 


\section{Enthalpy and Heat Capacity}

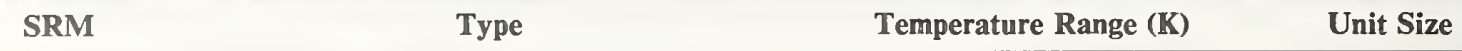

$\begin{array}{rlrr}705 & \text { Polystyrene, powder } & 10-350 & 5 \mathrm{~g} \\ 1475 & \text { Polyethylene, powder } & 5-360 & 50 \mathrm{~g}\end{array}$

\section{Differential Scanning Calorimetry}

These SRM's are for calibrating differential scanning calorimeters, differential thermal analyzers, and similar instruments.

\begin{tabular}{|c|c|c|c|c|}
\hline SRM & Type & $\begin{array}{c}\text { Melting } \\
\text { Temperature }\end{array}$ & Enthalpy of Fusion & Unit of Issue (mm) \\
\hline 2220 & Tin $(99.9995 \%)$ & $505.10 \mathrm{~K}$ & $\begin{array}{c}7147 \mathrm{~J} / \mathrm{mol} \\
(107.46 \mathrm{~J} / \mathrm{g})\end{array}$ & $25 \times 25 \times 0.127$ \\
\hline $2221 a$ & Zinc $(99.999 \%)$ & $692.74 \mathrm{~K}$ & $\begin{array}{l}7026 \mathrm{~J} / \mathrm{mol} \\
(60.22 \mathrm{~J} / \mathrm{g})\end{array}$ & $25 \times 25 \times 0.0508$ \\
\hline 2222 & Biphenyl (99.984\%) & $342.41 \mathrm{~K}$ & $120.41 \mathrm{~J} / \mathrm{g}$ & $1 \mathrm{~g}$ \\
\hline 2223 & Potassium Nitrate & IN PREP & & \\
\hline 2225 & Mercury & 234.30 & $11.469 \mathrm{~J} / \mathrm{g}$ & $2.5 \mathrm{~g}$ \\
\hline
\end{tabular}

This SRM is for evaluating methods of determining purity by differential scanning calorimetry. It consists of phenacatin and phenacetin doped with p-aminobenzoic acid.

\begin{tabular}{ccccc}
\hline SRM & Type & Dopant Level (p-ABA, mol\%) & Unit \\
\hline 1514 & Thermal Analysis Purity & $0,0.7,2,5$ & Set of $4,0.5 \mathrm{~g} /$ vial \\
\hline
\end{tabular}




\section{Differential Thermal Analysis}

GM's 754, 757, 758, 759, 760, and 761 have been issued by NIST in cooperation with the International Confederation of Thermal Analysis as standards for calibrating differential thermal analysis, differential scanning calorimetry, and thermogravimetry equipment under operating conditions.

\begin{tabular}{|c|c|c|c|c|}
\hline GM & Material & & Peak Temp. & Unit \\
\hline 754 & Polystyrene & (glass transition) & $105^{\circ} \mathrm{C}$ & $10 \mathrm{~g}$ \\
\hline 757 & $\begin{array}{l}\text { 1,2-Dichloroethane } \\
\text { Clycohexane }\end{array}$ & $\begin{array}{l}\text { (melting point) } \\
\text { (transition point) } \\
\text { (melting point) }\end{array}$ & $\begin{array}{r}-32^{\circ} \mathrm{C} \\
-83^{\circ} \mathrm{C} \\
7{ }^{\circ} \mathrm{C}\end{array}$ & $\begin{array}{l}4 \mathrm{~mL} \\
4 \mathrm{~mL}\end{array}$ \\
\hline & $\begin{array}{l}\text { Phenyl Ether } \\
\text { o-Terphenyl }\end{array}$ & $\begin{array}{l}\text { (melting point) } \\
\text { (melting point) }\end{array}$ & $\begin{array}{l}30^{\circ} \mathrm{C} \\
58^{\circ} \mathrm{C}\end{array}$ & $\begin{array}{l}4 \mathrm{~mL} \\
5 \mathrm{~g}\end{array}$ \\
\hline 758 & $\begin{array}{l}\text { Potassium Nitrate } \\
\text { Indium } \\
\text { Tin } \\
\text { Potassium Perchlorate } \\
\text { Silver Sulfate }\end{array}$ & $\begin{array}{l}\text { (transition point) } \\
\text { (melting point) } \\
\text { (melting point) } \\
\text { (transition point) } \\
\text { (transition point) }\end{array}$ & $\begin{array}{l}128^{\circ} \mathrm{C} \\
157^{\circ} \mathrm{C} \\
232{ }^{\circ} \mathrm{C} \\
300^{\circ} \mathrm{C} \\
430{ }^{\circ} \mathrm{C}\end{array}$ & $\begin{aligned} 10 \mathrm{~g} \\
3 \mathrm{~g} \\
3 \mathrm{~g} \\
10 \mathrm{~g} \\
3 \mathrm{~g}\end{aligned}$ \\
\hline 759 & $\begin{array}{l}\text { Potassium Perchlorate } \\
\text { Silver Sulfate } \\
\text { Quartz } \\
\text { Potassium Sulfate } \\
\text { Potassium Chromate }\end{array}$ & $\begin{array}{l}\text { (transition point) } \\
\text { (transition point) } \\
\text { (transition point) } \\
\text { (transition point) } \\
\text { (transition point) }\end{array}$ & $\begin{array}{l}300^{\circ} \mathrm{C} \\
430{ }^{\circ} \mathrm{C} \\
573{ }^{\circ} \mathrm{C} \\
583^{\circ} \mathrm{C} \\
665^{\circ} \mathrm{C}\end{array}$ & $\begin{aligned} 10 \mathrm{~g} \\
3 \mathrm{~g} \\
3 \mathrm{~g} \\
10 \mathrm{~g} \\
10 \mathrm{~g}\end{aligned}$ \\
\hline 760 & $\begin{array}{l}\text { Quartz } \\
\text { Potassium Sulfate } \\
\text { Potassium Chromate } \\
\text { Barium Carbonate } \\
\text { Strontium Carbonate }\end{array}$ & $\begin{array}{l}\text { (transition point) } \\
\text { (transition point) } \\
\text { (transition point) } \\
\text { (transition point) } \\
\text { (transition point) }\end{array}$ & $\begin{array}{l}573^{\circ} \mathrm{C} \\
583^{\circ} \mathrm{C} \\
665^{\circ} \mathrm{C} \\
810^{\circ} \mathrm{C} \\
925^{\circ} \mathrm{C}\end{array}$ & $\begin{array}{l}3 \mathrm{~g} \\
10 \mathrm{~g} \\
10 \mathrm{~g} \\
10 \mathrm{~g} \\
10 \mathrm{~g}\end{array}$ \\
\hline 761 & $\begin{array}{l}\text { Permanorm } 3 \\
\text { Nickel } \\
\text { Mumetal } \\
\text { Permanorm } 5 \\
\text { Trafoperm }\end{array}$ & $\begin{array}{l}\text { (magnetic transition) } \\
\text { (magnetic transition) } \\
\text { (magnetic transition) } \\
\text { (magnetic transition) } \\
\text { (magnetic transition) }\end{array}$ & $\begin{array}{l}259^{\circ} \mathrm{C} \\
353^{\circ} \mathrm{C} \\
381{ }^{\circ} \mathrm{C} \\
454^{\circ} \mathrm{C} \\
750^{\circ} \mathrm{C}\end{array}$ & $\begin{array}{ll}1 \mathrm{~g} \\
1 \mathrm{~g} \\
1 \mathrm{~g} \\
1 \mathrm{~g} \\
1 \mathrm{~g}\end{array}$ \\
\hline
\end{tabular}

\section{Superconductive Thermometric Fixed Point Device}

Each device is composed of small cylinders of high purity material mounted in a threaded copper stud and enclosed by a mutual inductance coil set. SRM 767a is intended to provide fixed points on the 1976 Provisional 0.5 to $30 \mathrm{~K}$ Temperature Scale (EPT-76). This SRM should prove particularly valuable to users of ${ }^{3} \mathrm{He}-{ }^{4} \mathrm{He}$ dilution refrigerators, in which direct calibrations on the liquid helium vapor pressuretemperature scales are difficult, and to those who wish to determine the temperature reproducibility of physical phenomena or of cryogenic equipment.

\begin{tabular}{|c|c|c|c|}
\hline SRM & Type & Material & $\begin{array}{c}\text { Nominal } \\
\text { Temperature }(\mathbf{K})\end{array}$ \\
\hline $767 \mathrm{a}$ & Superconductive Thermometric Fixed Point Device & $\begin{array}{l}\text { Niobium } \\
\text { Lead } \\
\text { Indium } \\
\text { Aluminum } \\
\text { Zinc } \\
\text { Cadmium }\end{array}$ & $\begin{array}{l}9.3 \\
7.2 \\
3.4 \\
1.2 \\
0.9 \\
0.5\end{array}$ \\
\hline
\end{tabular}




\section{Freezing Point}

SRM's 740a and 741 are defining fixed points for the International Practical Temperature Scale of 1968 (IPTS-68). The secondary reference points are for calibrating thermometers, thermocouples, and other temperature measuring devices. These SRM's are certified per IPTS-68.

\section{Defining Fixed Points}

\begin{tabular}{cllcc}
\hline \multicolumn{2}{c}{ SRM } & Type & Temperature ${ }^{\circ} \mathrm{C}$ & Wt/Unit (grams) \\
\hline & & & 419.58 & 350 \\
740 & Zinc & 231.9681 & 350 \\
\hline
\end{tabular}

\section{Secondary Reference Points}

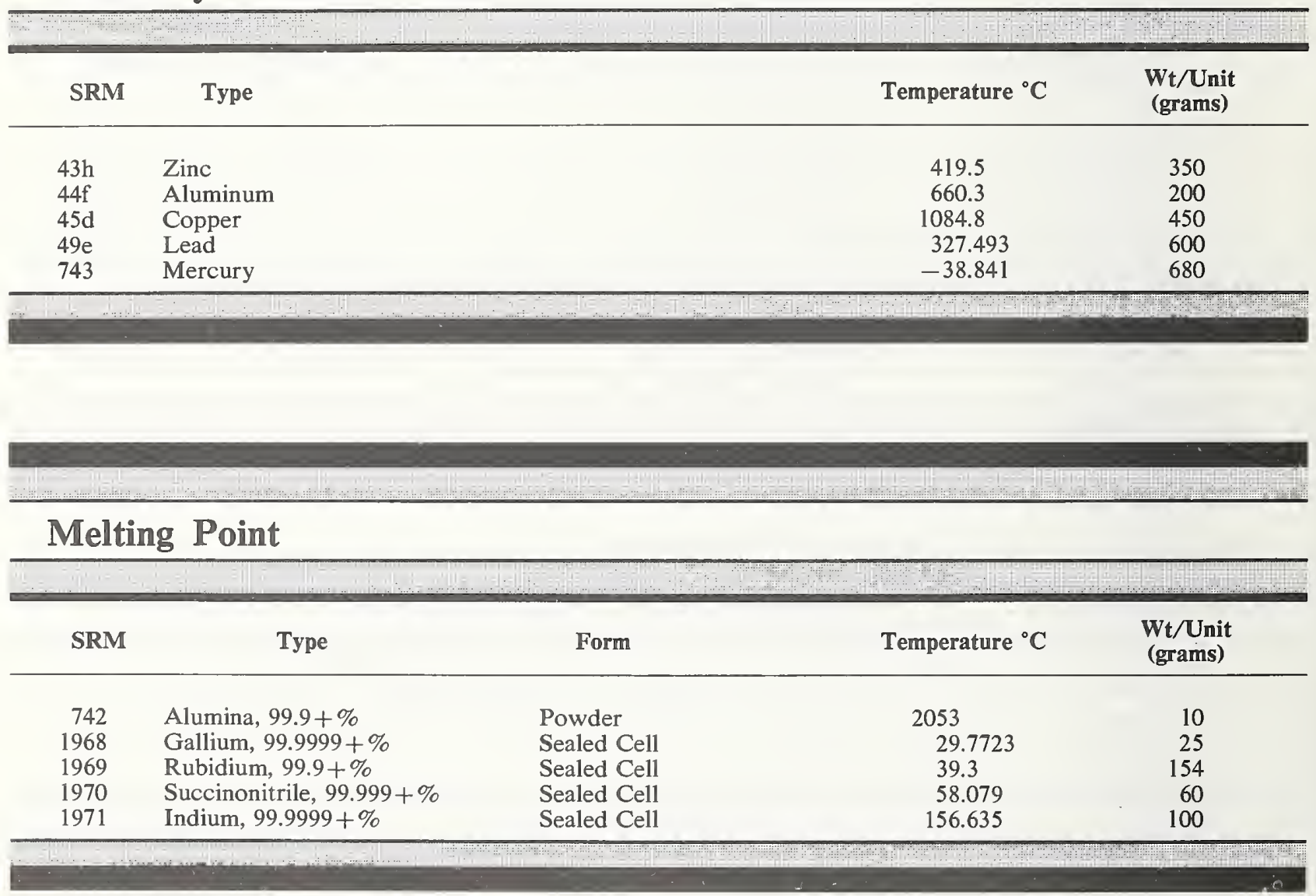


GM 8000 is issued by NIST in cooperation with the Office of Reference Materials at the National Physical Laboratory (NPL) in Teddington, England. This set of 10 highly purified substances is intended for use in the calibration of thermometry used in determining the melting points of samples in glass capillary tubes. Both the meniscus point and the liquefaction point for each substance are certified by NPL.

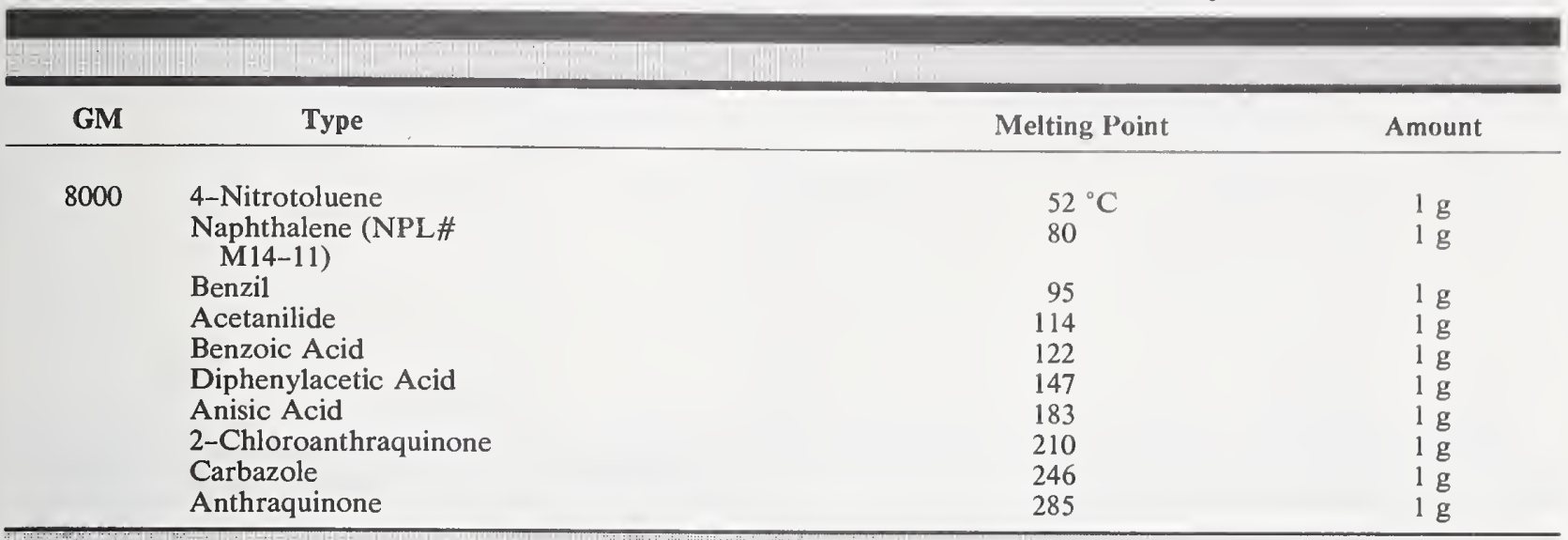

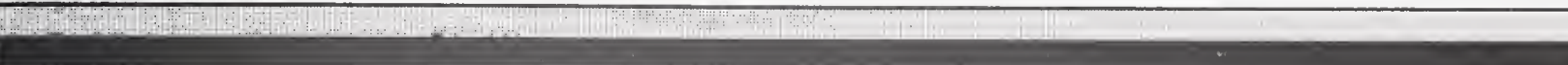

\section{Laboratory Thermometer}

This mercury-in-glass thermometer is for use in clinical laboratories. Its main scale extends from 24.00 to $38.00{ }^{\circ} \mathrm{C}$, in 0.05 in ${ }^{\circ} \mathrm{C}$ divisions. It has an auxiliary scale from -0.20 to $+0.20{ }^{\circ} \mathrm{C}$.

\begin{tabular}{ccccc}
\hline & Type & Calibrated Points $\left({ }^{\circ} \mathrm{C}\right)$ & Unit \\
\hline SRM & Clinical Laboratory Thermometer & $0,25,30,37$ & Each \\
\hline $934 \quad$ & & & \\
\hline
\end{tabular}

\section{Thermocouple Material}

SRM
Type

Temperature Range

$\left({ }^{\circ} \mathrm{C}\right)$

Form

1967 Platinum, High-Purity $(99.999+\%)$

197 to 1767

Wire. $0.51 \mathrm{~mm} \mathrm{D,} 1$ meter long

\section{Vapor Pressure}

\begin{tabular}{clcccc}
\hline SRM & Type & $\begin{array}{c}\text { Pressure Range } \\
\text { (atmosphere) }\end{array}$ & Temperature Range (K) & Unit Size \\
\hline 745 & Gold & $10^{-9}$ to $10^{-3}$ & $1300-2100$ & Wire $1.44 \mathrm{~mm} \times 152 \mathrm{~mm}$ \\
746 & Cadmium & $10^{-11}$ to $10^{-4}$ & $350-594$ & Rod $6.4 \mathrm{~mm} \times 64 \mathrm{~mm}$ \\
748 & Silver & $10^{-12}$ to $10^{-3}$ & $800-1600$ & Rod $6.4 \mathrm{~mm} \times 64 \mathrm{~mm}$ \\
\hline
\end{tabular}




\section{Thermal Conductivity}

\begin{tabular}{|c|c|c|c|c|}
\hline SRM & Type & Dimension (mm) & Temperature Range (K) & $\begin{array}{c}\text { Conductivity at } \\
293 \mathrm{~K}(\mathrm{~W} / \mathrm{M} \cdot \mathrm{K})\end{array}$ \\
\hline 1461 & Stainless Steel & $12.7 \mathrm{D}, 50$ length & $2-1200$ & 14.1 \\
\hline 1462 & Stainless Steel & $34 \mathrm{D}, 50$ length & $2-1200$ & 14.1 \\
\hline 8420 & Electrolytic Iron & $6.4 \mathrm{D}, 50$ length & $2-1000$ & 77.9 \\
\hline 8421 & Electrolytic Iron & $31.7 \mathrm{D}, 50$ length & $2-1000$ & 77.9 \\
\hline 8422 & Sintered Tungsten & $3.2 \mathrm{D}, 50$ length & $2-3000$ & 173 \\
\hline 8423 & Sintered Tungsten & $6.4 \mathrm{D}, 50$ length & $2-3000$ & 173 \\
\hline 8424 & Graphite & $6.4 \mathrm{D}, 50$ length & $5-2500$ & 90.9 \\
\hline 8425 & Graphite & $12.7 \mathrm{D}, 50$ length & $5-2500$ & 90.9 \\
\hline 8426 & Graphite & $25.4 \mathrm{D}, 50$ length & $5-2500$ & 90.9 \\
\hline
\end{tabular}

\section{Thermal Expansion}

$\begin{array}{lllll}\text { SRM Type } & \text { Temperature Range (K) Diameter }(\mathrm{mm}) \quad \text { Length }(\mathrm{mm})\end{array}$

\begin{tabular}{llrrr}
$731-\mathrm{L} 1$ & Borosilicate Glass & $80-680$ & 6.4 & 51 \\
$731-\mathrm{L} 2$ & Borosilicate Glass & $80-680$ & 6.4 & 102 \\
$731-\mathrm{L} 3$ & Borosilicate Glass & $80-680$ & 6.4 & 152 \\
738 & Stainless Steel (AISI 446) & $293-780$ & 6.4 & 51 \\
$739-\mathrm{L} 1$ & Fused Silica & $80-1000$ & 6.4 & 51 \\
\hline $739-\mathrm{L} 2$ & Fused Silica & $80-1000$ & 6.4 & 102 \\
$739-\mathrm{L} 3$ & Fused Silica & $80-1000$ & 6.4 & 152 \\
\hline
\end{tabular}

\section{Thermal Resistance}

SRM Type Dimension (mm)

$600 \times 600 \times 25.4$
$600 \times 600 \times 25.4$
$600 \times 600 \times 25.4$
$600 \times 600 \times 25.4$
$300 \times 300 \times 25.4$

$600 \times 600 \times 25.4$

Filica Board

Fibrous Glass Blanket

Fibrous Glass Blanket

Fumed Silica Board
Thermal

Resistance

Temperature Range (K)

at $293 \mathrm{~K}$ $\left(\mathrm{m}^{2} \cdot \mathrm{K} / \mathrm{W}\right)$

$\begin{array}{cl}297.1 & 1.2 \\ 100-330 & 0.75 \\ 100-330 & 0.60 \\ 297.1 & 0.60 \\ 297.1 & 1.2\end{array}$




\section{Magnetic}

\section{Magnetic Susceptibility}

\begin{tabular}{|c|c|c|c|}
\hline \multirow[t]{2}{*}{ SRM } & \multirow[t]{2}{*}{ Type } & $\begin{array}{c}\text { Gram Susceptibility } \\
\text { @297 K }\end{array}$ & \multirow[t]{2}{*}{ Form/Unit } \\
\hline & & $\left(10^{6} \times \mathrm{g}, \mathrm{cm}^{3} \cdot \mathrm{g}^{-1}\right)$ & \\
\hline $\begin{array}{l}763 \\
766\end{array}$ & $\begin{array}{l}\text { Aluminum } \\
\text { Manganese Fluoride }\end{array}$ & $\begin{array}{l}0.604 \\
123.3\end{array}$ & $\begin{array}{l}\text { Cylinder } 3 \mathrm{~mm} \text { diameter } \times 3 \mathrm{~mm} \\
\text { Cube } 3 \times 3 \times 3 \mathrm{~mm}\end{array}$ \\
\hline
\end{tabular}

\section{Magnetic Moment}

\begin{tabular}{|c|c|c|c|c|}
\hline \multirow{2}{*}{ SRM } & \multirow{2}{*}{ Type } & \multirow{2}{*}{ Magnetic, $\mathrm{H}(\mathrm{Oe})$} & Moment, $\sigma$ & \multirow{2}{*}{ Size } \\
\hline & & & $\left(\mathrm{Oe} \cdot \mathrm{cm}^{3} \cdot \mathrm{g}^{-1}\right)$ & \\
\hline 772 & Nickel Sphere & $3500-10,000$ & $54.75-54.90$ & $2.4 \mathrm{~mm} \mathrm{D}$ \\
\hline
\end{tabular}

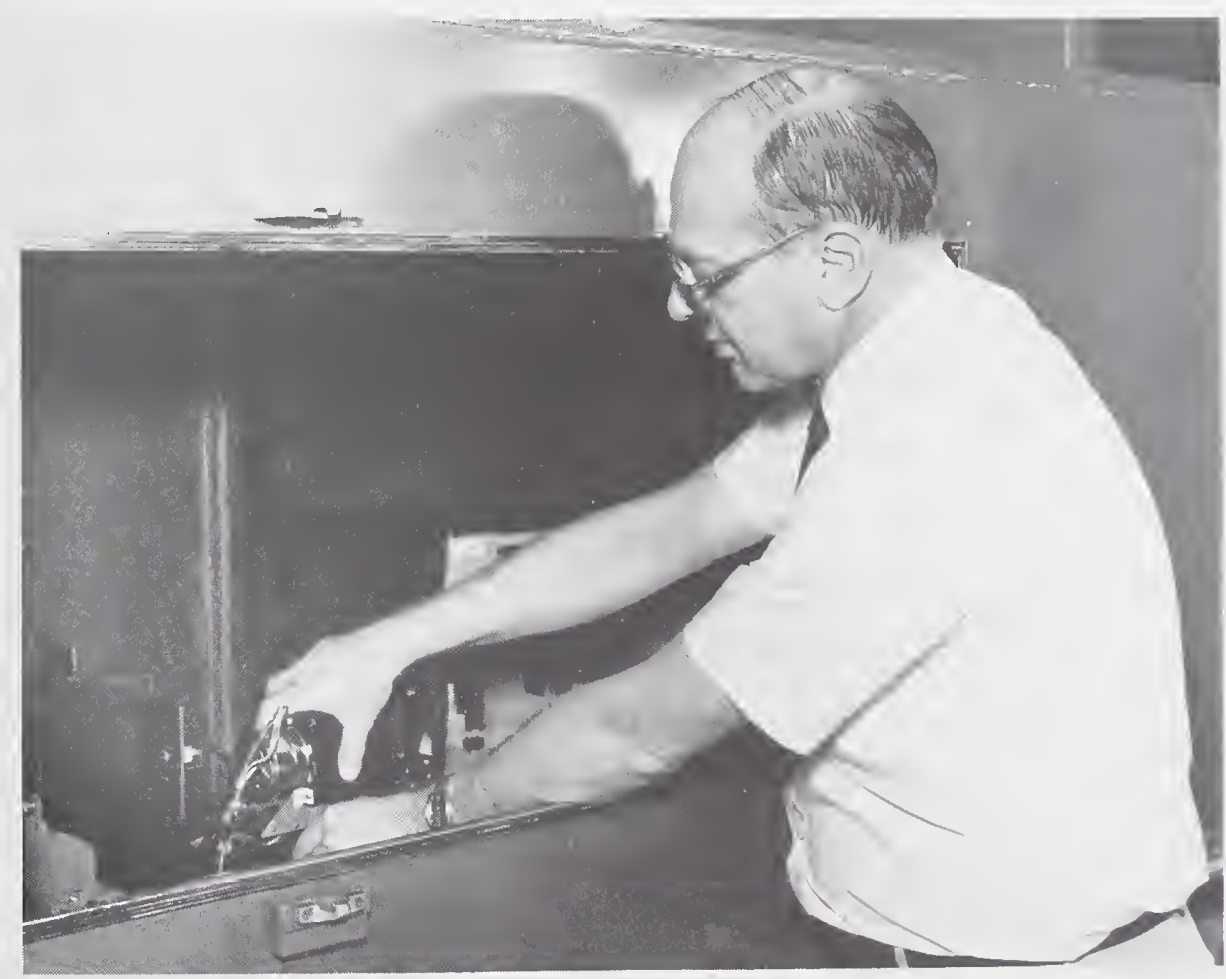

Ken Eckerle of the Radiometric Physics Division is shown here making an adjustment to a component of the high accuracy spectrophotometer in preparation for measurements of one of the optical properties SRM's. 


\section{OPTICAL}

\section{Spectrophotometric}

SRM 930D: This SRM consists of three neutral density glass filters. The filters have transmittances of approximately 10, 20, and 30 percent. Each filter is individually certified for transmittance at wavelengths of $440,465,546.1,590$, and $635 \mathrm{~nm}$.

SRM 931d: This SRM consists of three sets of four solutions-a blank solution and three concentrations of absorbing liquid. The net absorbances are certified for each concentration at wavelengths of $302,395,512$, and $678 \mathrm{~nm}$.

SRM 935a: Solutions made with this SRM are certified for apparent specific absorbances at wavelengths of $235,257,313,345$, and $350 \mathrm{~nm}$.

SRM 936: A solution made with this SRM is certified for its molecular emission spectrum over the wavelength range of 375 to $675 \mathrm{~nm}$.

SRM 1930: This SRM consists of three neutral density glass filters. The filters have transmittances of approximately 1,3, and 50 percent. Each filter is individually certified for transmittance at wavelengths of $440,465,546.1,590$, and $635 \mathrm{~nm}$.

SRM 1931: This SRM is a set of four luminescent samples and a blank certified for corrected luminescence emission spectra over the wavelength range from 400 to $760 \mathrm{~nm}$. Each sample consists of an inorganic dye in a sintered polytetrafluoroethylene matrix mounted in a cuvette-sized holder.

SRM 2009a: This SRM is for checking the wavelength scale between 400 and $760 \mathrm{~nm}$ for bandpasses between 1.5 and $10.5 \mathrm{~nm}$. SRM 2009a is mounted in a standard cuvette-sized holder.

SRM 2031: This SRM consists of three filters mounted in holders and an empty holder; all holders are equipped with shutters. Two of the filters have an evaporated layer of semi-transparent metal sandwiched between two quartz plates assembled by optical contact. The third filter is a single quartz plate. Each filter is individually calibrated at $250,280,340,360,400,465,500,546.1,590$, and $635 \mathrm{~nm}$.

SRM 2032: Aqueous solutions made with this SRM are certified for specific absorbances from 240 to 280 $\mathrm{nm}$ for use as a stray light standard in the ultraviolet region.

SRM 2033: This SRM consists of the same material as SRM 2032 plus a reference beam attenuator for extending the dynamic range of the stray light test.

SRM 2034: This SRM is a solution sealed in a non-fluorescent, fused-silica cuvette for checking the wavelength scale between 240 and $650 \mathrm{~nm}$.

\begin{tabular}{clcc}
\hline SRM & \multicolumn{1}{c}{ Type } & $\begin{array}{c}\text { Wavelength Range } \\
\text { (nm) }\end{array}$ & Unit \\
\hline & & & \\
$930 D$ & Glass Filters, Transmittance & $440-635$ & 3 filters/4 holders \\
$931 \mathrm{~d}$ & Liquid Filters, Absorbance & $302-678$ & Set: \\
$935 \mathrm{a}$ & Potassium Dichromate, UV Absorbance & $235-350$ & 15 grams \\
936 & Quinine Sulfate Dihydrate, Fluorescence & $375-675$ & 1 gram \\
1930 & Glass Filters, Transmittance & $440-635$ & 3 filters/4 holders \\
\hline 1931 & Fluorescence Corrected Emission Spectra & $400-760$ & Set: 4 pieces \\
$2009 a$ & Didymium-oxide Glass, Wavelength & $400-760$ & 3 filter/1 holder \\
2031 & Metal-on-Quartz Filters, Transmittance & $250-635$ & 3 filters/4 holders \\
2032 & Potassium Iodide, Stray Light & $240-280$ & 25 grams \\
2033 & Potassium Iodide with Attenuator & $240-280$ & 25 grams w/attenuator \\
2034 & Holmium-oxide Solution, Wavelength & $240-650$ & 1 sealed cuvette \\
\hline
\end{tabular}




\section{Reflectance}

These SRM's are for calibrating the reflectance scale of integrating sphere reflectometers used to evaluate materials for solar energy collectors and to calibrate reflectometers used in evaluating the appearance of polished metals and metal plated objects.

\section{Specular Spectral Reflectance}

\begin{tabular}{llrl}
\hline \multicolumn{1}{c}{ SRM } & \multicolumn{1}{c}{ Type } & Wavelength Range (nm) & Size \\
\hline & & & \\
2003 & First Surface, Aluminum on Glass & $250-2500$ & $5.1 \mathrm{~cm} \mathrm{D}$ \\
2011 & First Surface, Gold on Glass & IN PREP & $5.1 \mathrm{~cm} \mathrm{D}$ \\
$2023 a$ & Second Surface, Aluminum on Fused Quartz & IN PREP & $5.1 \mathrm{~cm} \mathrm{D}$ \\
2025 & $\begin{array}{l}\text { Second Surface, Aluminum on Fused Quartz with } \\
\text { Wedge }\end{array}$ & 250-2500 & $2.5 \times 10.2 \mathrm{~cm}$ \\
\hline
\end{tabular}

\section{Infrared Reflectance}

This SRM is for use in establishing the accuracy of the wavelength scale of reflectance spectrophotometers.

\begin{tabular}{cccc}
\hline & Type & Wavelength Range (nm) & Size \\
\hline SRM & Near IR Wavelength & $740-2000$ & $51 \mathrm{~mm} \mathrm{D} \times 12 \mathrm{~mm}$ \\
\hline 1920 & &
\end{tabular}

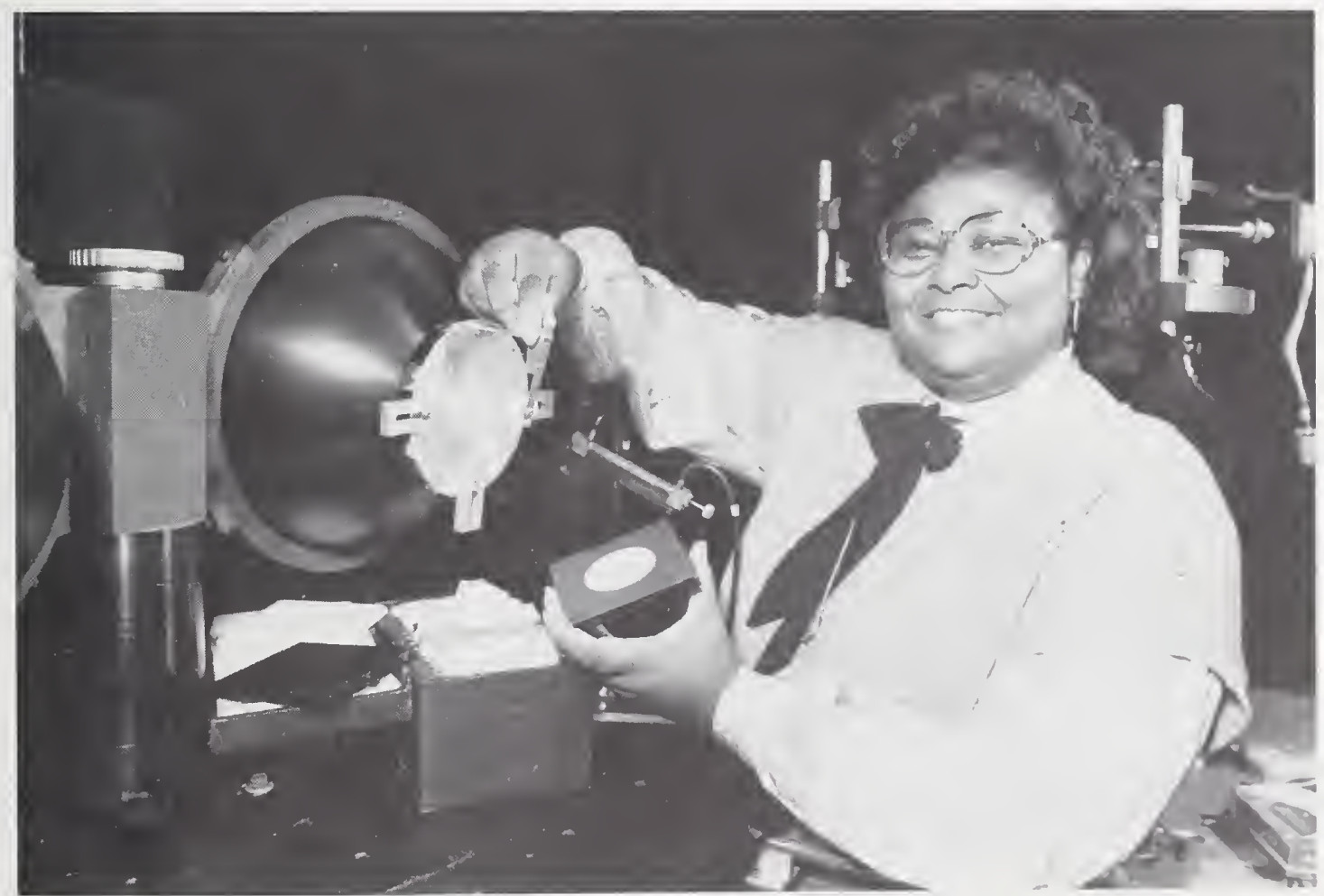

Yvonne Barnes prepares to mount a sample on the reference spectral reflectometer to make certification measuremenis on a reflectance SRM. 


\section{Directional-Hemispherical Reflectance}

\begin{tabular}{lllll}
\hline SRM & \multicolumn{1}{c}{ Type } & Wavelength Range & Size \\
(nm) & & & \\
2015 & Opal Glass & $400-750$ & $2.5 \times 5.0 \times 0.64 \mathrm{~cm}$ \\
2016 & Opal Glass & $400-750$ & $10 \times 10 \times 0.64 \mathrm{~cm}$ \\
2021 & Black Porcelain Enamel & $280-2500$ & $5.1 \times 5.1 \times 0.20 \mathrm{~cm}$ \\
\hline
\end{tabular}

\section{Refractive Index}

SRM's 211c, 2211, and 2213 are certified for refractive index at 20, 25, and $30^{\circ} \mathrm{C}$, from 435.8 to $667.8 \mathrm{~nm}$ for seven wavelengths.

SRM 1822 is certified for refractive index at thirteen wavelengths from $404.7 \mathrm{~nm}$ to $706.5 \mathrm{~nm}$. This SRM is designed for calibrating refractometers and certifying refractive index immersion liquids. It consists of two rectangular glass slabs: one slab has polished faces and is to be used to check the performance of a refractometer; the second slab is unpolished and can be broken into fragments to certify the refractive index of immersion liquids by microscope methods.

SRM 1823 consists of two silicone liquids that are miscible and span the refractive index range of a variety of glasses and glass fibers. The liquids are suitable for calibrating refractometers and are certified for refractive index at ten wavelengths from 435.8 to $667.8 \mathrm{~nm}$, at temperatures of $20,40,60$, and $80{ }^{\circ} \mathrm{C}$.

\begin{tabular}{|c|c|c|c|}
\hline SRM & Type & $\mathrm{n}^{20}, \lambda 546.1$ & Unit Size \\
\hline $211 \mathrm{c}$ & Toluene & 1.5008 & $5 \mathrm{~mL}$ \\
\hline 2211 & Toluene & 1.5008 & $8 \mathrm{~mL}$ \\
\hline 2213 & 2,2,4-Trimethylpentane (Isooctane) & 1.3934 & $25 \mathrm{~mL}$ \\
\hline $1823-\mathrm{I}$ & Silicone Liquid (I) & 1.5214 & $60 \mathrm{~mL}$ \\
\hline $1823-$ II & Silicone Liquid (II) & 1.5638 & $60 \mathrm{~mL}$ \\
\hline
\end{tabular}

\section{Optical Rotation}

These SRM's are intended for use in calibrating or checking polarimetric apparatus. In aqueous solution the optical rotation of SRM 17d is certified at three wavelengths, while that of SRM 41c is certified at two wavelengths. SRM 4lc is also certified at one wavelength in a dimethyl sulfoxide solution.

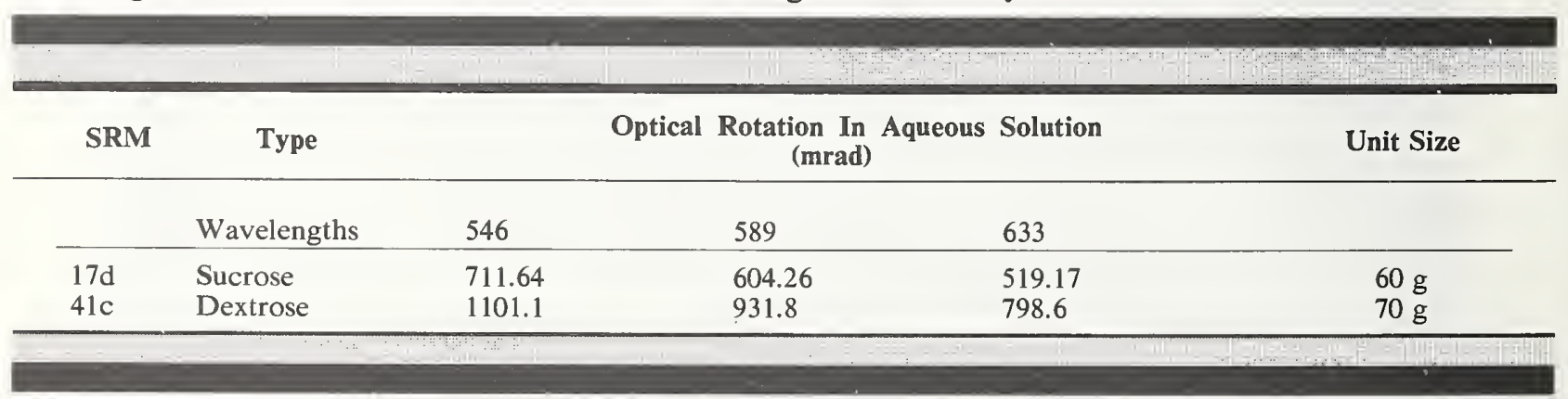




\section{RADIOACTIVITY}

These SRM's are shipped express or air freight (shipping charges collect). The amount of a radionuclide in an SRM, at a specified time, is stated as (1) the number of atoms (or the mass, for radium SRM's), (2) the activity, or "decays per second," or (3) the emission rate of a particular radiation, depending on the method of calibration or the intended use. For solution SRM's, the quantity is usually specified per gram of liquid. The active portion of gamma-ray "point-source" standards is usually restricted to the central few millimeters of a low-mass, low- $Z$ support to minimize scattering. Alpha-particle-emitting radionuclides are deposited or plated on metal backings.

The unit for activity has traditionally been the curie (Ci), but simpler relations between activity, emission rate, and counting rate result if the current SI (International System of Units) unit "1 per second"

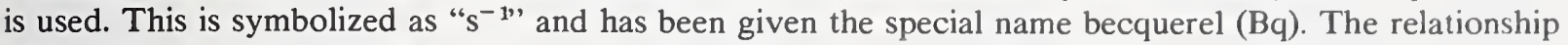
between the curie and the becquerel is:

$1 \mathrm{Ci}=3.7 \times 10^{10} \mathrm{~Bq}$.

Many SRM's are measured and certified in terms of emission rate. In this catalog, $\alpha \mathrm{s}^{-1}, \beta^{-} \mathrm{s}^{-1}, \beta^{+} \mathrm{s}^{-1}$, $\mathrm{Kxs}^{-1}$, and $\gamma \mathrm{s}^{-1}$ are used for the emission rates of alpha particles, negatrons, positrons, $\mathrm{K} \mathrm{x}$ rays, and gamma rays, respectively.

The SRM's without an asterisk $\left({ }^{*}\right)$ may be ordered singly, without a license, under the general licensing provisions of the Atomic Energy Act of 1954. Those marked by an asterisk are available only under the special licensing provisions of the Atomic Energy Act of 1954.

NOTE: Certain radionuclides are not economical to maintain in stock because of short half lives or low demand. When sufficient demand exists, based on letters of inquiry, these materials are prepared and those who have expressed interest are notified of their availability. If you need any radionuclide not listed, write the Radioactivity Group, Room C114 Radiation Physics Building, National Institute of Standards and Technology, Gaithersburg, MD 20899; or telephone (301) 975-5531.

In addition, chemically stable solutions of most radionuclides may be submitted to NIST for calibration as described in Calibration Services Users Guide, NIST Special Publication 250 (1986-88/ Revised ed.). Requests for such tests should be submitted, with full source information, for approval of suitability to the Radioactivity Group.

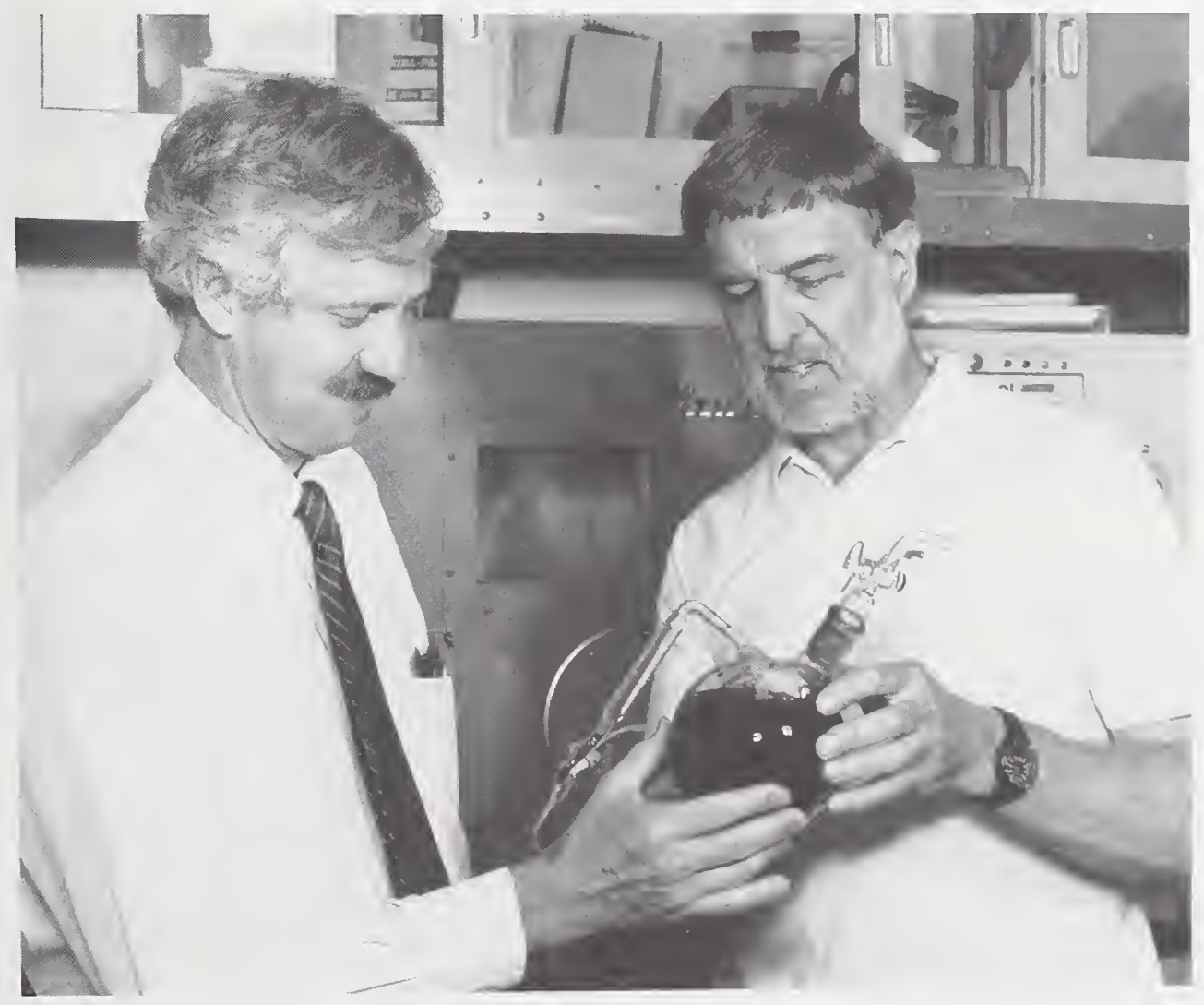

Bert Coursey and Bill McLaughlin of the Ionizing Radiation Division discuss the preparation and measurement of a radioactivity sample. 


\section{Alpha-Particle, Beta-Particle, Gamma-Ray, and Electron-Capture Solutions}

SRM Radionuclide $\quad \begin{gathered}\text { Approximate activity, per gram, at time } \\ \text { of calibration (month/year) }\left(\mathrm{Bq} \mathbf{g}^{-1}\right)\end{gathered}$

$\begin{array}{cc}\text { Approx. } & \text { Overall } \\ \text { Mass of } & \text { Uncertainty } \\ \text { Solution }(\mathrm{g}) & (\%)\end{array}$

\begin{tabular}{|c|c|c|c|c|c|}
\hline $\begin{array}{l}4322^{*} \\
4332 C^{*} \\
4251 B^{*} \\
4233 C^{*} \\
4943^{*}\end{array}$ & $\begin{array}{l}\text { Americium-241 } \\
\text { Americium-243 } \\
\text { Barium-133 } \\
\text { Cesium-137-Barium-137m } \\
\text { Chlorine-36 }\end{array}$ & $\begin{array}{l}38.7 \\
89 \\
5 \times 10^{5} \\
7 \times 10^{5} \\
1 \times 10^{4}\end{array}$ & $\begin{array}{r}11 / 86 \\
9 / 89 \\
1 / 82 \\
8 / 89 \\
12 / 84\end{array}$ & $\begin{array}{l}5 \\
5 \\
5 \\
5 \\
3\end{array}$ & $\begin{array}{l}1.0 \\
1.4 \\
1.4 \\
1.4 \\
0.8\end{array}$ \\
\hline $\begin{array}{l}4915 D^{*} \\
4320 \\
4329^{*} \\
4370^{*} \\
4926 D\end{array}$ & $\begin{array}{l}\text { Cobalt-60 } \\
\text { Curium-244 } \\
\text { Curium-243 } \\
\text { Europium-152 } \\
\text { Hydrogen-3 }\end{array}$ & $\begin{array}{l}3 \times 10^{5} \\
57 \\
70 \\
9 \times 10^{4} \\
3 \times 10^{3}\end{array}$ & $\begin{array}{l}3 / 84 \\
4 / 89 \\
6 / 84 \\
2 / 87 \\
5 / 89\end{array}$ & $\begin{array}{r}5 \\
5 \\
5 \\
5 \\
18\end{array}$ & $\begin{array}{l}0.8 \\
1.0 \\
1.4 \\
1.1 \\
0.6\end{array}$ \\
\hline $\begin{array}{l}\text { 4927D } \\
4947 \mathrm{C} \\
4361 \mathrm{~B} \\
4949 \mathrm{~B} \\
4929 \mathrm{D}\end{array}$ & $\begin{array}{l}\text { Hydrogen-3 } \\
\text { Hydrogen-3 } \\
\text { Hydrogen-3 } \\
\text { Iodine-129 } \\
\text { Iron-55 }\end{array}$ & $\begin{array}{l}7 \times 10^{5} \\
3 \times 10^{5} \\
1.1 \\
7 \times 10^{3} \\
4 \times 10^{4}\end{array}$ & $\begin{array}{l}4 / 89 \\
3 / 87 \\
8 / 87 \\
1 / 82 \\
8 / 85\end{array}$ & $\begin{array}{r}3 \\
4 \\
490 \\
1 \\
5\end{array}$ & $\begin{array}{l}0.8 \\
1.2 \\
1.0 \\
1.9 \\
2.6\end{array}$ \\
\hline $\begin{array}{l}4226 \mathrm{~B}^{*} \\
4327^{*} \\
4326 \\
4323^{*} \\
4338^{*}\end{array}$ & $\begin{array}{l}\text { Nickel-63 } \\
\text { Polonium-208 } \\
\text { Polonium-209 } \\
\text { Plutonium-238 } \\
\text { Plutonium-240 }\end{array}$ & $\begin{array}{l}1 \times 10^{6} \\
77 \\
370 \\
33 \\
18\end{array}$ & $\begin{array}{r}12 / 84 \\
6 / 84 \\
9 / 89 \\
11 / 86 \\
4 / 80\end{array}$ & $\begin{array}{l}4 \\
1.1 \\
5 \\
5 \\
5\end{array}$ & $\begin{array}{l}1.1 \\
1.4 \\
2.0 \\
0.5 \\
1.0\end{array}$ \\
\hline $\begin{array}{l}4940 \mathrm{C} \\
4423 \\
4919 \mathrm{~F}^{*} \\
4288^{*} \\
4328^{*}\end{array}$ & $\begin{array}{l}\text { Promethium-147 } \\
\text { Strontium-90 } \\
\text { Strontium-90 } \\
\text { Technetium-99 } \\
\text { Thorium-229 }\end{array}$ & $\begin{array}{l}1 \times 10^{5} \\
4 \times 10^{6} \\
4 \times 10^{3} \\
4 \times 10^{4} \\
884\end{array}$ & $\begin{array}{r}8 / 85 \\
11 / 85 \\
5 / 88 \\
11 / 82 \\
5 / 84\end{array}$ & $\begin{array}{l}5 \\
5 \\
5 \\
5 \\
2\end{array}$ & $\begin{array}{l}0.4 \\
1.1 \\
1.2 \\
1.6 \\
1.5\end{array}$ \\
\hline $\begin{array}{l}4324^{*} \\
4321\end{array}$ & $\begin{array}{l}\text { Uranium-232 } \\
\text { Uranium-238 (Natural) }\end{array}$ & $\begin{array}{l}83 \\
263\end{array}$ & $\begin{array}{r}2 / 84 \\
11 / 86\end{array}$ & $\begin{array}{l}5 \\
5\end{array}$ & $\begin{array}{l}1.5 \\
0.4\end{array}$ \\
\hline $4276 C^{*}$ & $\begin{array}{l}\text { Long-Lived Mixed Radionuclide: } \\
\text { Antimony-125 } \\
\text { Europium-154 } \\
\text { Europium-155 }\end{array}$ & $\begin{array}{l}2 \times 10^{4} \\
1 \times 10^{4} \\
7 \times 10^{3}\end{array}$ & $9 / 88$ & 5 & \\
\hline
\end{tabular}

*License certification is required by NIST for these radionuclides. 


\section{Alpha-Particle Point-Sources}

These SRM's consist of a practically weightless deposit of the nuclide on a thin platinum foil cemented to a monel disk.

\begin{tabular}{|c|c|c|c|c|}
\hline SRM & Radionuclide & \multicolumn{2}{|c|}{$\begin{array}{l}\text { Approx. } \alpha \text {-particle-emission rate into } \\
2 \pi \text { geometry and/or approx. activity at } \\
\text { time of calibration (month/year) }\end{array}$} & $\begin{array}{l}\text { Overall uncertainty } \\
(\%)\end{array}$ \\
\hline 4904NG* & Americium-241 & $2300 \mathrm{~Bq} \mathrm{~g}^{-1}$ & $5 / 86$ & 1.3 \\
\hline 4904SG* & Americium-241 & $2300 \mathrm{~Bq} \mathrm{~g}^{-1}$ & $5 / 86$ & 1.0 to 1.3 \\
\hline $4906 \mathrm{HC}^{*}$ & Plutonium-238 & $1 \times 10^{3}-5 \times 10^{4}$ & $10 / 87$ & 1.3 \\
\hline $4906 C^{*}$ & Plutonium-238 & $1 \times 10-3 \times 10^{2}$ & $10 / 87$ & 1.3 \\
\hline
\end{tabular}

*License certification is required by NIST for these radionuclides.

:

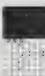

Radiocarbon Dating and Ground Water Studies

Contemporary Standard for Carbon-14 Dating Laboratories

\begin{tabular}{ccc}
\hline SRM & Material & Description \\
\hline 4990C $\quad$ Oxalic Acid & $\begin{array}{l}\text { One-half pound of oxalic acid taken from specially prepared } \\
\text { material for use as a common contemporary standard against } \\
\text { which world-wide measurements can be compared. }\end{array}$
\end{tabular}

Low-Level Tritiated-Water Standard

\begin{tabular}{ccc}
\hline SRM & Material & \multicolumn{1}{c}{ Description } \\
\hline 4361B & Hydrogen-3 & $\begin{array}{l}\text { Contains } 490 \text { grams of }{ }^{3} \mathrm{H}-\mathrm{H}_{2} \mathrm{O} \text { in a flame-sealed bottle. The } \\
\text { radioactivity concentration was } 1.123 \mathrm{~Bq} \mathrm{~g}^{-1} \text {, as of the date of } \\
\text { the most recent gas-counting measurement-August } 1,1987 \text {. The } \\
\text { total uncertainty in this value is } 1.0 .\end{array}$ \\
\hline
\end{tabular}
the most recent gas-counting measurement-August 1, 1987. The thetal uncertainty in this 1.0

\section{Gaseous Materials}

\begin{tabular}{|c|c|c|c|c|c|c|}
\hline SRM & Radionuclide & $\begin{array}{l}\text { Approximate activ } \\
\text { radioactivity concentrat } \\
\text { of calibration (mont }\end{array}$ & $\begin{array}{l}\text { or } \\
\text { at time } \\
\text { ear) }\end{array}$ & $\begin{array}{c}\text { Approx. } \\
\text { Vol. }\left(\mathrm{cm}^{3}\right)\end{array}$ & $\begin{array}{l}\text { Approx. } \\
\text { Pressure } \\
\text { (atm) }\end{array}$ & $\begin{array}{c}\text { Overall } \\
\text { Uncertainty } \\
(\%)\end{array}$ \\
\hline $4935 C^{*}$ & Krypton-85 & $5 \times 10^{7} \mathrm{~Bq} \mathrm{~mol}^{-1}$ & $3 / 74$ & 10 & 1 & 0.9 \\
\hline $4235 B^{*}$ & Krypton-85 & $1 \times 10^{7} \mathrm{~Bq}$ & $10 / 86$ & 3 & 1 & 1.1 \\
\hline $4308 \mathrm{C}$ & Krypton- 85 & $2 \times 10^{6} \mathrm{~Bq}$ & $11 / 82$ & 30 & 0.3 & 3.2 \\
\hline $4415 \mathrm{LN}^{*}$ & Xenon-133 & $5 \times 10^{8} \mathrm{~Bq}$ & $3 / 90$ & 5 & 0.1 & 1.5 \\
\hline
\end{tabular}

* License certification is required by NIST for these radionuclides. 


\section{Gamma-Ray Solutions}

These samples are contained in flame-sealed glass ampoules.

\begin{tabular}{ccccc}
\hline & & & \\
\hline SRM & $\begin{array}{c}\text { Nominal Radium Content } \\
(\mathrm{g})\end{array}$ & Approx. Mass of Solution & $\begin{array}{c}\text { Overall } \\
(\mathrm{g})\end{array}$ & $\begin{array}{c}\text { Uncertainty } \\
(\%)\end{array}$ \\
\hline & & & & \\
4956 & $2 \times 10^{-7}$ & & 5 & 4.4 \\
4957 & $5 \times 10^{-7}$ & $9 / 67$ & 5 & 1.8 \\
4958 & $1 \times 10^{-6}$ & $9 / 67$ & 5 & 1.8 \\
4959 & $2 \times 10^{-6}$ & $9 / 67$ & 5 & 1.3 \\
\hline
\end{tabular}

\section{Environmental Natural Matrix Materials for Quality Assurance Testing}

\section{SRM 4350B-Columbia River Sediment}

This material was collected from a river downstream from a nuclear reactor facility. Concentrations of fission and activation products are elevated over typical world-wide levels. ${ }^{239 / 240} \mathrm{Pu}$ and ${ }^{241} \mathrm{Am}$ are very homogeneously distributed through the sample and are in acid-leachable forms. Inhomogeneity is 3 percent or better for other radionuclides.

\section{SRM 4351-Human Lung}

This material contains radioactivity concentrations on the order of $10^{-4} \mathrm{~Bq} \mathrm{~g}^{-1}$. It has been freeze-dried, cryogenically ground, homogenized, and packed in a glass bottle under vacuum. There is significant inhomogeneity in ${ }^{239 / 240} \mathrm{Pu}$ which is unavoidable because plutonium was taken into the lungs in particulate form. Assessments of accuracy of measurement technique can be improved by averaging over several samples.

\section{SRM 4352-Human Liver}

This material contains radioactivity concentrations on the order of $10^{-4} \mathrm{~Bq} \mathrm{~g}^{-1}$. It has been freeze-dried, cryogenically ground, homogenized, and packed in a glass bottle under vacuum.

\section{SRM 4353-Rocky Flats Soil Number 1}

This material was collected within 13 centimeters of the soil surface at Rocky Flats, CO. ${ }^{239 / 240} \mathrm{Pu}$ and ${ }^{240}$ Am concentrations are about an order of magnitude higher than typical world-wide levels. Approximately 10 percent of the plutonium is in a acid-resistant form. The material also contains "hot" particles and a statistical method is provided for dealing with these. Inhomogeneities, excluding hot particles, are on the order of 3 percent or better.

\section{SRM 4354-Freshwater Lake Sediment}

This material (gyttja) contains approximately 25 grams of freeze-dried, pulverized freshwater lake sediment (approximately 50 percent organic by weight) in a polyethylene bottle. The SRM is intended for use in tests of measurements of environmental radioactivity contained in matrices similar to the sample, for evaluating analytical methods, or as a generally available calibrated "real" sample matrix in interlaboratory comparisons.

\section{SRM 4355-Peruvian Soil}

This material has non-measurable radioactivity concentrations for many fallout radionuclides and can be used as a blank or for sensitive tests of radioanalytical procedures at low-radioactivity concentrations for other radionuclides. The results of a trace-element study are given for 57 elements. 


\section{Radiopharmaceuticals}

\begin{tabular}{|c|c|c|c|c|c|}
\hline SRM & $\begin{array}{l}\text { Radionuclide } \\
\text { (5 mL solution) }\end{array}$ & $\begin{array}{l}\text { Half } \\
\text { Life }\end{array}$ & & $\begin{array}{c}\text { Approximate } \\
\text { Radioactivity at } \\
\text { Time of Dispatch } \\
\left(\mathbf{B q} \mathbf{g}^{-1}\right)\end{array}$ & $\begin{array}{c}\text { Overall } \\
\text { Uncertainty }\end{array}$ \\
\hline 4400 LK* & Chromium-51 & 27.702 & d & $3 \times 10^{6}$ & 0.7 \\
\hline $4416 \mathrm{LK}^{*}$ & Gallium-67 & 3.261 & d & $3 \times 10^{6}$ & 0.8 \\
\hline $4421 \mathrm{~L}^{*}$ & Gold-195 & 183 & d & $5 \times 10^{5}$ & 2.3 \\
\hline $4405 \mathrm{LB}^{*}$ & Gold-198 & 2.696 & d & $4 \times 10^{6}$ & 1.7 \\
\hline 4417LI* & Indium-111 & 2.805 & d & $5 \times 10^{6}$ & 0.7 \\
\hline 4414LC* & Iodine-123 & 13.221 & $\mathrm{~h}$ & $6 \times 10^{7}$ & 1.5 \\
\hline $4407 \mathrm{LN}^{*}$ & Iodine-125 & 59.6 & d & $1 \times 10^{6}$ & 1.0 \\
\hline 4401LP* & Iodine-131 & 8.021 & d & $5 \times 10^{6}$ & 0.9 \\
\hline $4411 \mathrm{LB}^{*}$ & Iron-59 & 44.51 & d & $8 \times 10^{5}$ & 1.5 \\
\hline 4420 LB* $^{*}$ & Lead-203 & 51.88 & $\mathrm{~h}$ & $3 \times 10^{6}$ & 1.0 \\
\hline $4418 L^{*}$ & Mercury-203 & 46.60 & d & $1 \times 10^{6}$ & 1.0 \\
\hline 4412LO* & Molybdenum-99-Technetium-99m & 65.92 & $\mathrm{~h}$ & $2 \times 10^{6}$ & 1.0 \\
\hline $4406 \mathrm{LJ} *$ & Phosphorus-32 & 14.29 & d & $2 \times 10^{6}$ & 1.7 \\
\hline 4409LD* & Selenium-75 & 119.8 & d & $1 \times 10^{6}$ & 2.8 \\
\hline $4403 \mathrm{LB}^{*}$ & Strontium-85 & 64.854 & d & $1 \times 10^{6}$ & 1.4 \\
\hline $4410 \mathrm{HO}^{*}$ & Technetium-99m & 6.007 & $\mathrm{~h}$ & $1 \times 10^{9}$ & 0.9 \\
\hline $4404 L^{*}$ & Thallium-201 & 72.91 & $\mathrm{~h}$ & $4 \times 10^{6}$ & 1.6 \\
\hline 4402 LC $^{*}$ & Tin-113-Indium-113m & 115.08 & d & $1 \times 10^{6}$ & 3.1 \\
\hline $4415 \mathrm{LN}^{*}$ & Xenon-133 ( 5 mL gas) & 5.243 & $\mathrm{~d}$ & $5 \times 10^{8} \mathrm{~s}^{-1}$ total & 1.0 \\
\hline 4419LC* & Ytterbium-169 & 32.03 & d & $2 \times 10^{6}$ & 1.3 \\
\hline
\end{tabular}

*License certification is required by NIST for these radionuclides.

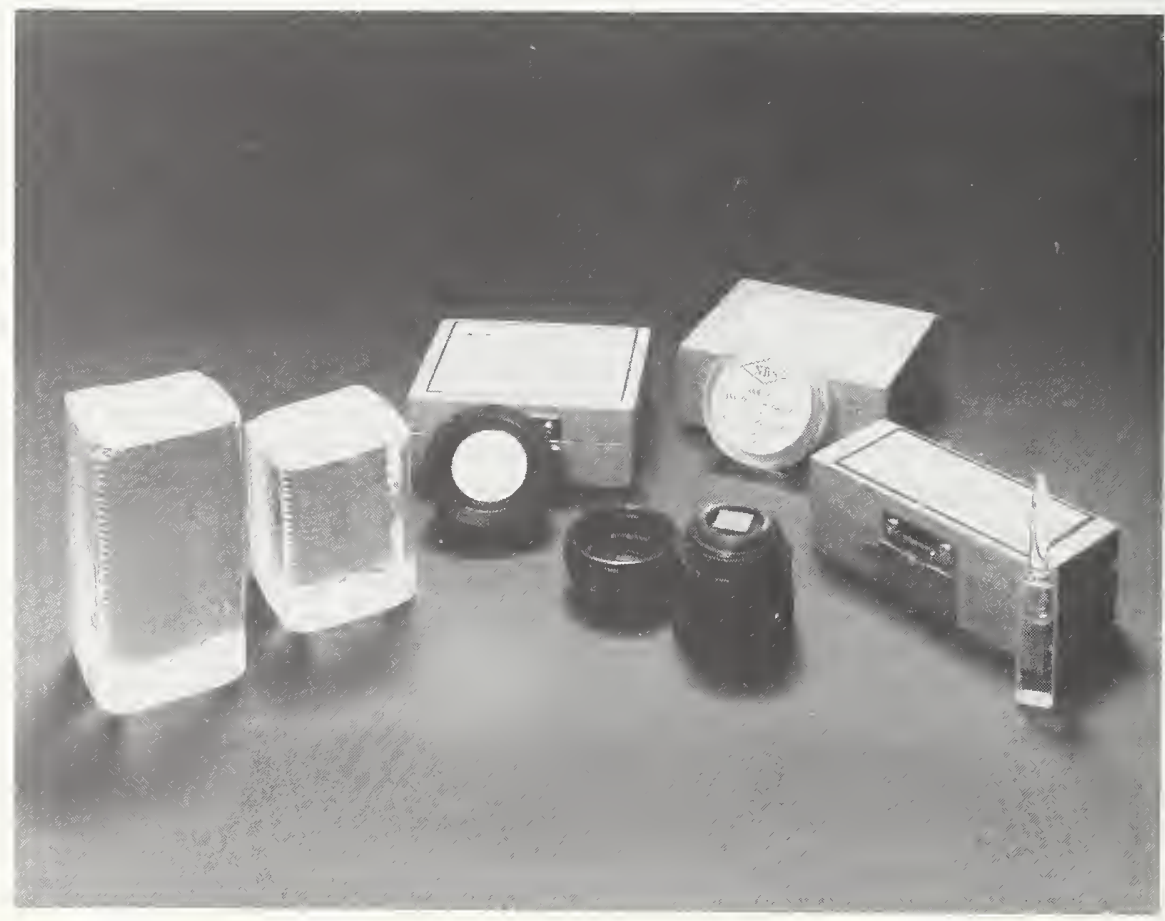

This series of SRM's, ranging from glass composition to spectral wavelength to microhardness, represent some of the diversity of the SRM's now available. 


\section{Metallurgical}

SRM's 485a, 486, 487, and 488 are for calibrating x-ray diffraction equipment used in determining the amount of retained austenite in ferrous materials. SRM 493 is for calibrating x-ray diffraction and Mössbauer equipment to determine the relative amounts of iron carbide in steel.

\begin{tabular}{|c|c|c|}
\hline SRM & Type & Form \\
\hline $485 \mathrm{a}$ & Austenite in Ferrite & Disk: $21 \mathrm{~mm}$ dia $\times 24 \mathrm{~mm}$ thick \\
\hline 486 & Austenite in Ferrite & Disk: $21 \mathrm{~mm}$ dia. $\times 2.4 \mathrm{~mm}$ thick \\
\hline 487 & Austenite in Ferrite & Disk: $21 \mathrm{~mm}$ dia. $\times 2.4 \mathrm{~mm}$ thick \\
\hline 488 & Austenite in Ferrite & Disk: $21 \mathrm{~mm}$ dia. $\times 2.4 \mathrm{~mm}$ thick \\
\hline 489 & Ferrite in Austenite & IN PREP \\
\hline 493 & Spheroidized Iron Carbide $\left(\mathrm{Fe}_{3} \mathrm{C}\right)$ in Ferrite & Wafer: $29 \times 29 \times 2.4 \mathrm{~mm}$ \\
\hline
\end{tabular}

\section{Abrasive Wear}

SRM 1857 is for use in the dry sand/rubber wheel abrasion test per ASTM G65, Procedure A.

\begin{tabular}{ccc}
\hline & & \\
\hline SRM & Type & Form \\
\hline $1857 \quad$ D-2 Tool Steel & 2 blocks: $7.8 \times 25 \times 76 \mathrm{~mm}$ \\
\hline
\end{tabular}

\section{Corrosion}

\section{Electrochemical Potential and Thickness}

This SRM is for determining the reliability of step test measurements of electrochemical and thickness of multilayered nickel deposits. It consists of a $50 \times 50 \mathrm{~mm}$ plate of copper-plated steel over which a duplex nickel coating has been deposited.

\begin{tabular}{|ccccc}
\hline SRM & Type & $\begin{array}{c}\text { Step Test Potential } \\
(\mathbf{m V})\end{array}$ & \multicolumn{2}{c}{$\begin{array}{c}\text { Nickel Thicknesses } \\
\text { Total Bright Semibright } \\
\text { (micrometers) }\end{array}$} \\
\hline 2350 & Nickel Step Test Standard & $110-150$ & 27 & (7) \\
\hline
\end{tabular}




\section{Pitting or Crevice Corrosion}

These SRM's are for use in evaluating the pitting or crevice corrosion of surgical implant materials per ASTM F746.

\begin{tabular}{|c|c|c|}
\hline SRM & Туре & Form \\
\hline 1890 & 316L Stainless Steel Rod and Teflon Collar & 4 sets: $6.4 \mathrm{~mm} \mathrm{D,} 25.4 \mathrm{~mm}$ long \\
\hline 1891 & Co-Cr-Mo Alloy Rod and Teflon Collar & 2 sets: $6.4 \mathrm{~mm} \mathrm{D}, 25.4 \mathrm{~mm}$ long \\
\hline
\end{tabular}

\section{X-ray Fluorescent Emission Target}

This SRM is intended for use in determining the detector window absorption in semiconductor x-ray spectrometers according to ANSI-IEEE Standard STO 759. When excited by a ${ }^{55} \mathrm{Fe}$ source this glass target will emit fluorescent $\mathrm{x}$ rays in the range 1.0 to $5.2 \mathrm{keV}$.

\begin{tabular}{cccccc}
\hline SRM & Type & & Form & Unit Size \\
\hline 477 & Glass Fluorescence Source & Disk & $2 \times 25$ mm D \\
\hline
\end{tabular}

\section{X-ray Diffraction}

These SRM's are powdered materials to be used as internal standards for powder diffraction measurements. SRM 674a is a set of five oxides for use in the quantitative analysis (intensity measurement) of materials. See also: SRM's 485a-488, 493 (p. 104), and SRM 1878 (p. 62).

\begin{tabular}{|c|c|c|c|}
\hline SRM & Type & $\begin{array}{l}\text { Lattice Parameter } \\
\left(25.0^{\circ} \mathrm{C}\right)\end{array}$ & Unit Size \\
\hline $640 \mathrm{~b}$ & Silicon Powder & $5.430940 \AA$ & $7.5 \mathrm{~g}$ \\
\hline $\begin{array}{l}656 \\
658 \\
659 \\
660 \\
\end{array}$ & $\begin{array}{l}\text { Alpha/Beta Standard } \\
\text { Tridymite } \\
\text { Ceramic Particle Size } \\
\text { LaB }_{6}-2 \text { Theta X-Ray Profile }\end{array}$ & $\begin{array}{l}\text { IN PREP } \\
\text { IN PREP } \\
\text { IN PREP } \\
4.15695 \AA\end{array}$ & $\begin{array}{l}5 \mathrm{~g} \\
3 \mathrm{~g}\end{array}$ \\
\hline $674 a$ & $\begin{array}{l}\text { Powder Diffraction Intensity } \\
\mathrm{Al}_{2} \mathrm{O}_{3} \text { ( } \alpha \text {-alumina) } \\
\mathrm{CeO}_{2} \\
\mathrm{Cr}_{2} \mathrm{O}_{3} \\
\mathrm{TiO}_{2} \text { (Rutile) } \\
\mathrm{ZnO}\end{array}$ & $\begin{array}{l}4.75893 \AA \\
5.41129 \AA \\
4.95916 \AA \\
4.59365 \AA \\
3.24981 \AA\end{array}$ & $\begin{array}{l}10 \mathrm{~g} \\
10 \mathrm{~g} \\
10 \mathrm{~g} \\
10 \mathrm{~g} \\
10 \mathrm{~g}\end{array}$ \\
\hline $\begin{array}{c}675 \\
676 \\
677 \\
\text { RM } 8585\end{array}$ & $\begin{array}{l}\text { Powder Diffraction (Mica) } \\
\text { Crystalline Alpha Alumina } \\
\text { Crystalline X-Ray Line Processing } \\
\text { Kaolin Based Relative XRD }\end{array}$ & $\begin{array}{l}9.98104 \AA \\
\text { IN PREP } \\
\text { IN PREP } \\
\text { IN PREP }\end{array}$ & $5 \mathrm{~g}$ \\
\hline
\end{tabular}




\section{Gas Transmission}

SRM 1470 is for use in the measurement of gas transmission rates using a volumetric method (ASTM D1434), manometric method (ASTM D1434), or coulometric method (ASTM D3985) of measurement. The permeances of nitrogen, oxygen, carbon dioxide, and helium through this polyester film at $296.15 \mathrm{~K}$ are

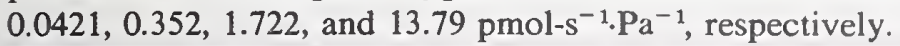

\begin{tabular}{ccc}
\hline TrM & Type & Unit Size \\
\hline $1470 \quad$ Polyester Plastic Film for Gas Transmission & 15 sheets, $23 \mathrm{~cm}$ square \\
\hline
\end{tabular}

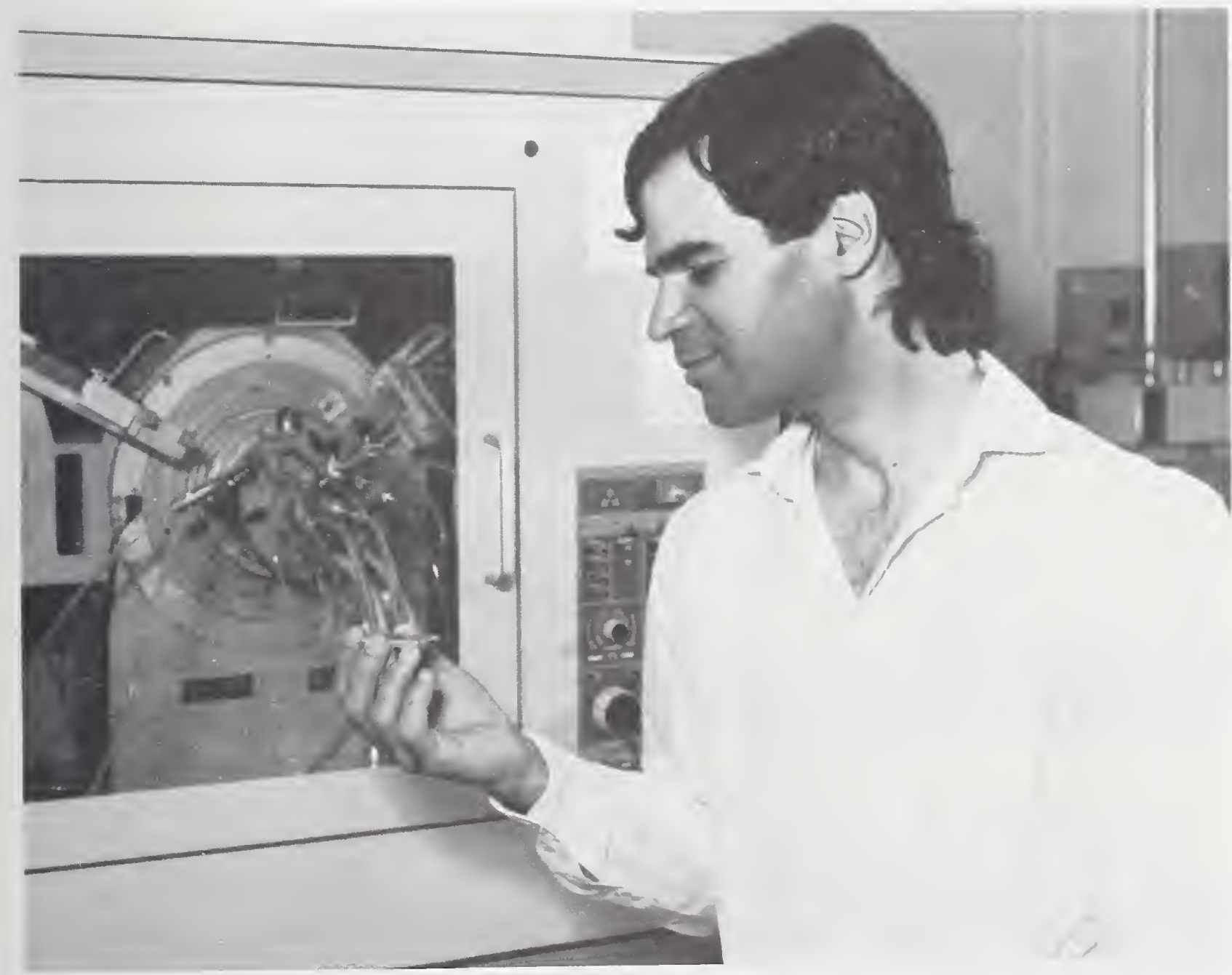

Jim Cline prepares to insert a X-ray diffraction candidate SRM material into a diffractometer. 


\section{Reference Fuel}

SRM's 1815a and 1816a are high purity liquids intended for use in maintaining the integrity of the octane rating of motor and aviation fuels as specified in the ASTM Manual for Rating Motor, Diesel and Aviation Fuels.

\begin{tabular}{|c|c|c|c|}
\hline SRM & Type & Purity, \% & Unit Size \\
\hline $1815 a$ & n-Heptane & 99.987 & $100 \mathrm{~mL}$ \\
\hline $1816 a$ & Isooctane $(2,2,4$-Trimethylpentane $)$ & 99.987 & $100 \mathrm{~mL}$ \\
\hline
\end{tabular}

\section{Electrical Resistivity and Conductivity}

\section{Metals}

These materials are for evaluating methods of measuring electrical resistance over wide temperature ranges.

\begin{tabular}{|c|c|c|c|c|}
\hline SRM & Type & Temperature Range & Resistivity at $293 \mathrm{~K}$ & Form \\
\hline 1461 & Stainless Steel & 5 to $1200 \mathrm{~K}$ & $80.5 \mu \Omega \cdot \mathrm{cm}$ & Rod: $12.7 \mathrm{~mm} \mathrm{D,} 50 \mathrm{~mm}$ long \\
\hline 1462 & Stainless Steel & 5 to $1200 \mathrm{~K}$ & $80.5 \mu \Omega \cdot \mathrm{cm}$ & Rod: $34.0 \mathrm{~mm} \mathrm{D}, 50 \mathrm{~mm}$ long \\
\hline 8420 & Iron & 6 to $1000 \mathrm{~K}$ & $10.1 \mu \Omega \cdot \mathrm{cm}$ & Rod: $\quad 6.4 \mathrm{~mm} \mathrm{D}, 50 \mathrm{~mm}$ long \\
\hline 8422 & Tungsten & 4 to $3000 \mathrm{~K}$ & $5.4 \mu \Omega \cdot \mathrm{cm}$ & $3.2 \mathrm{~mm} \mathrm{D}, 50 \mathrm{~mm}$ long \\
\hline 8423 & Tungsten & 4 to $3000 \mathrm{~K}$ & $5.4 \mu \Omega \cdot \mathrm{cm}$ & $6.4 \mathrm{~mm} \mathrm{D}, 50 \mathrm{~mm}$ long \\
\hline
\end{tabular}

\section{Silicon}

SRM's 1521, 1522, and 1523 sets of Resistivity Standards will be replaced with SRM 2541-50 series of Resistivity Standards as they become available. This series will be $100 \mathrm{~mm}$ in diameter and will have improved accuracy and precision. SRM's 2526, 2527, 2528, and 2529 are mounted on beveling blocks for two-probe test equipment.

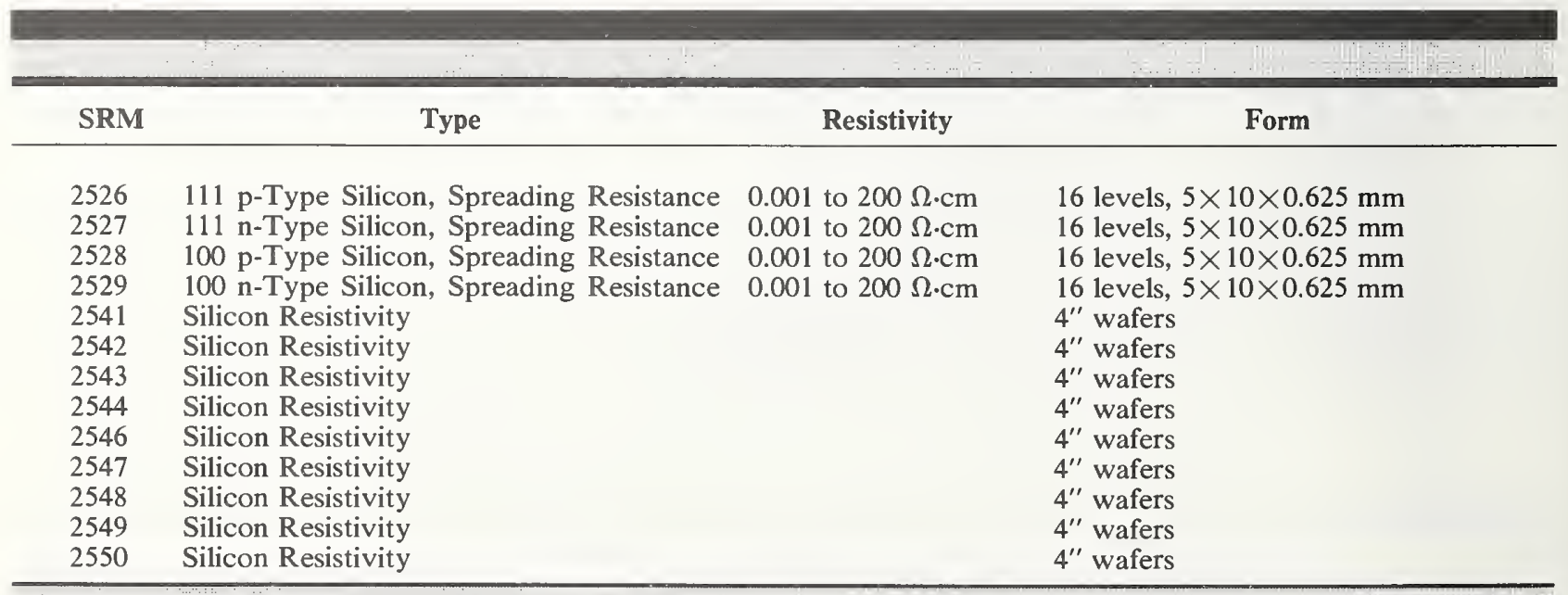




\section{Residual Resistivity Ratio}

This SRM is a set of five aluminum rods that are intended for use in checking four-terminal dc and eddy current decay techniques. The residual resistivity ratio, $\rho(273 \mathrm{~K}) / \rho(4 \mathrm{~K})$, is a sensitive indicator of purity and of the mechanical state of a material.

\begin{tabular}{|c|c|c|c|}
\hline SRM & Type & RRR Values & Form \\
\hline 769 & Aluminum & $130,683,1205,2650$, and 11,000 & $6.4 \mathrm{~mm} \mathrm{D,} 52 \mathrm{~mm}$ long \\
\hline
\end{tabular}

\section{Eddy Current}

These SRM's are intended for use in the calibration of eddy current conductivity meters and of secondary electrical conductivity standards. Eddy current measurements are used in nondestructive inspection of conducting materials and in the sorting of alloys for composition and heat treatment.

\begin{tabular}{clll}
\hline & & & \\
\hline \multicolumn{1}{c}{ Type } & Conductivity & Form \\
\hline 1860 & Aluminum & & \\
1862 & Aluminum-Magnesium Alloy & $60 \%$ IACS & $44 \times 44 \times 9.5 \mathrm{~mm}$ \\
\hline
\end{tabular}

\section{Electrolytic Conductance}

These SRM's are for calibrating and standardizing conductivity cells and meters used in water purity determinations. They are solutions of high-purity potassium chloride in de-ionized water in equilibrium with atmospheric carbon dioxide.

\begin{tabular}{cccc}
\hline SRM & Type & $\begin{array}{c}\text { Nominal Conductance } \\
\text { (microsiemens per cm, } \boldsymbol{\mu S} / \mathbf{c m})\end{array}$ & Unit Size \\
\hline 3191 & Electrolytic Conductance & 100 & $500 \mathrm{~mL}$ \\
3192 & Electrolytic Conductance & 500 & $500 \mathrm{~mL}$ \\
3193 & Electrolytic Conductance & 1000 & $500 \mathrm{~mL}$ \\
3194 & Electrolytic Conductance & 10000 & $500 \mathrm{~mL}$ \\
3195 & Electrolytic Conductance & 100000 & $500 \mathrm{~mL}$ \\
\hline
\end{tabular}

\section{Superconducting Critical Current}

This SRM is for checking the performance of measurement systems used in superconductor technology. It consists of $2.2 \mathrm{~m}$ of a multifilamentary niobium titanium, copper stabilized superconducting wire wound in a single layer onto a spool with a core diameter of $8.7 \mathrm{~cm}$.

\begin{tabular}{|c|c|c|c|}
\hline SRM & Type & Magnetic Field (T) & Critical Current (A) \\
\hline 1457 & $\mathrm{Nb}-\mathrm{Ti}$ Wire & $\begin{array}{l}2.000 \\
4.000 \\
6.000 \\
8.000\end{array}$ & $\begin{array}{r}293.30 \\
187.38 \\
124.72 \\
69.72\end{array}$ \\
\hline
\end{tabular}



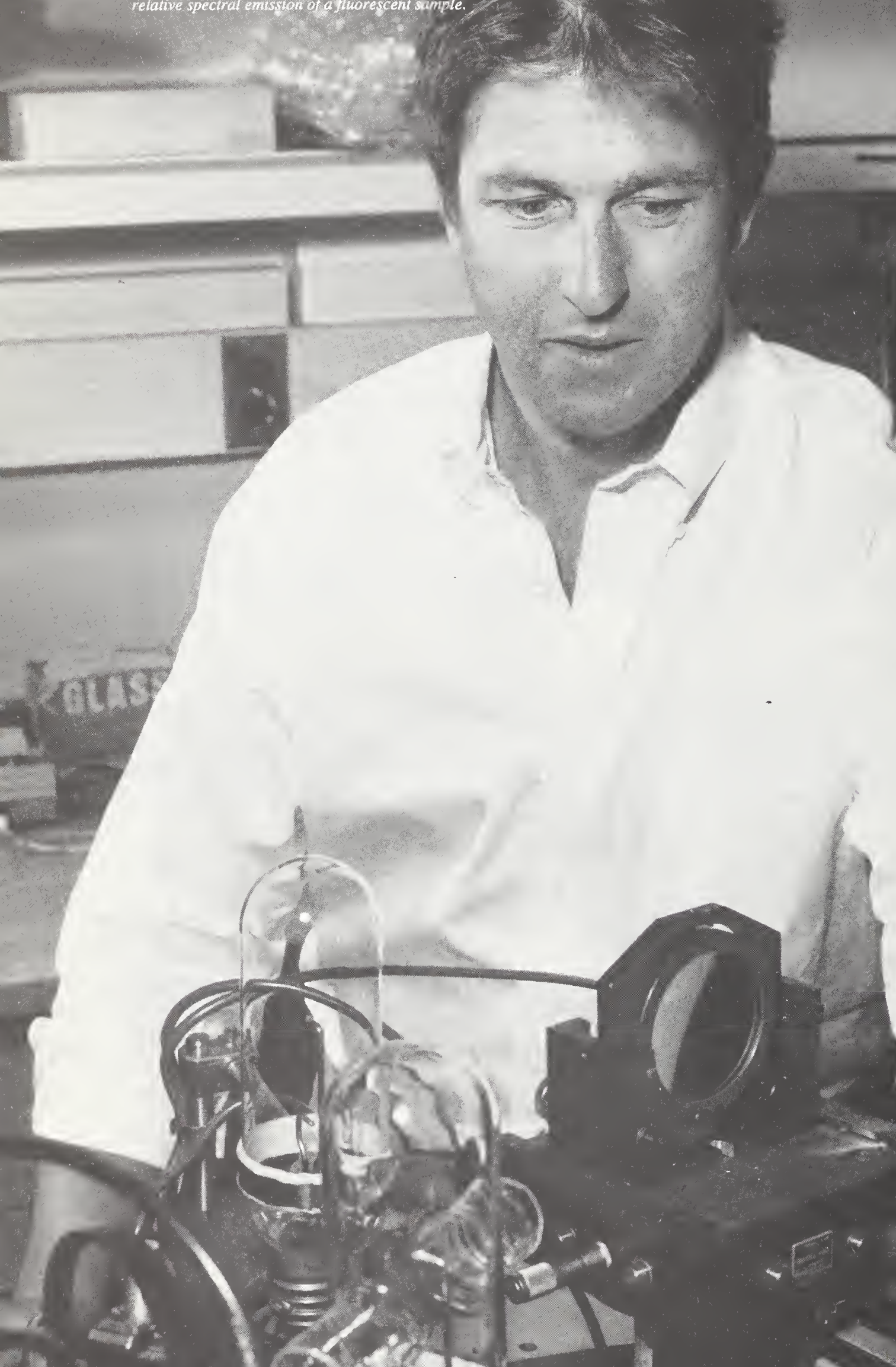

1)
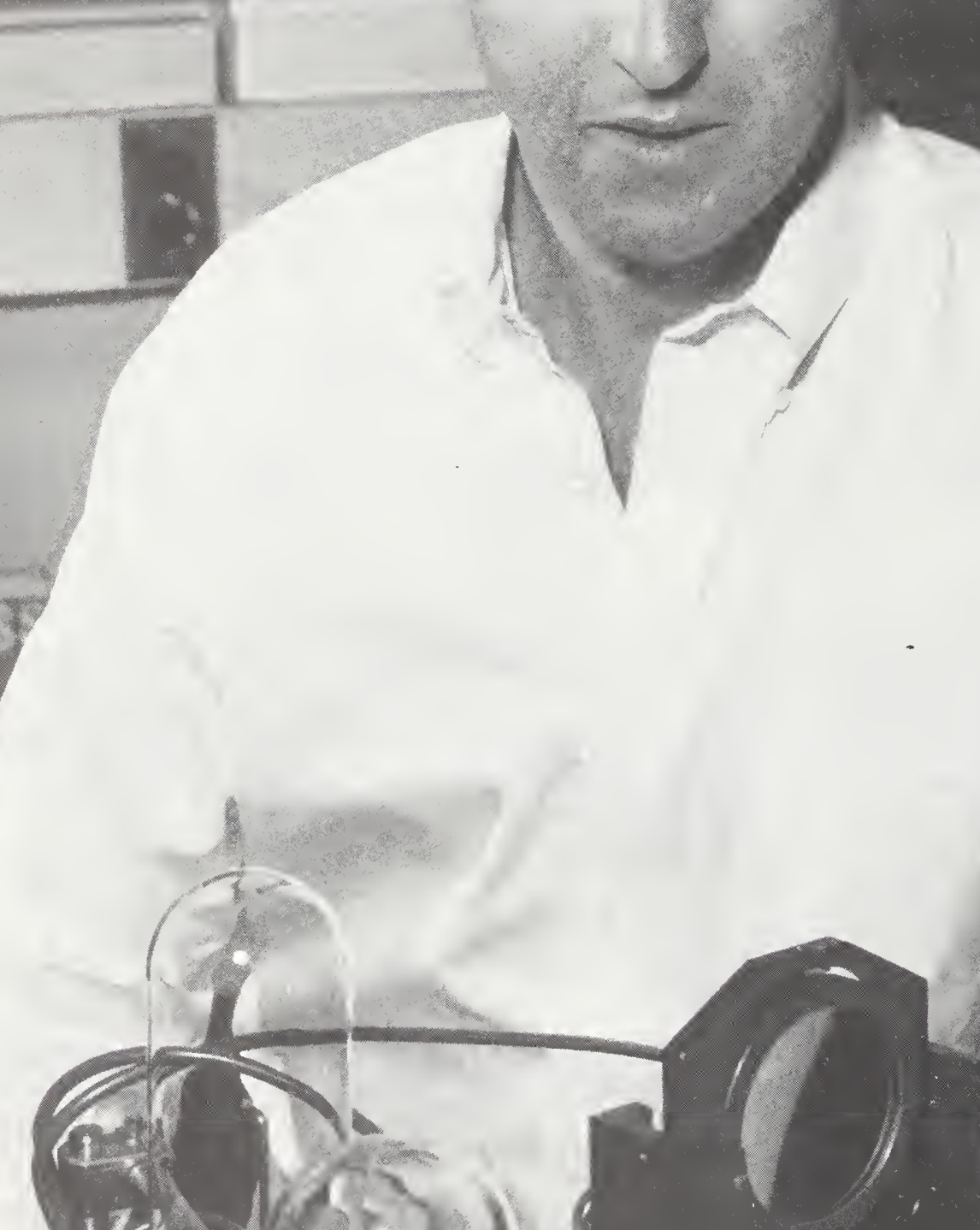


\section{Engineering Materials}

\section{Standard Rubbers and Rubber-Compounding Materials}

These SRM's have been prepared to provide the rubber industry with standard materials for rubber compounding. They are useful for the testing of rubber and rubber-compounding materials in connection with quality control of raw materials and for the standardization of rubber testing.

Each material has been statistically evaluated for uniformity by mixing rubber and rubber compounds, and vulcanizing them in accordance with ASTM Designation D-15 and determining the stressstrain properties of the resulting vulcanizates. Certificates are issued for the rubbers because the properties of different lots are not the same. Replacement lots of rubber-compounding SRM's impart essentially the same characteristics to rubber vulcanizates so that Certificates are not issued for these SRM's.

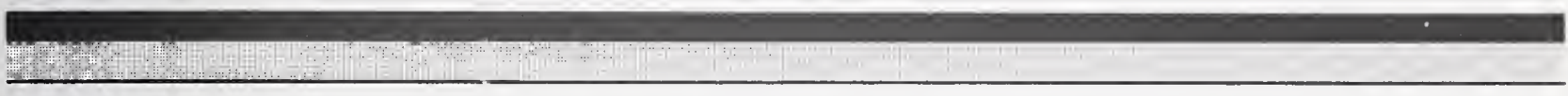

\section{Rubbers}

\begin{tabular}{|c|c|c|c|}
\hline SRM & Type & Wt/Unit & Pounds \\
\hline $386 \mathrm{k}$ & Styrene-butadiene 1500 & $34 \mathrm{~kg}$ & 75 \\
\hline $388 \mathrm{p}$ & Butyl & $34 \mathrm{~kg}$ & 75 \\
\hline $1495^{\mathrm{P}}$ & Butyl (Low Viscosity) & $34 \mathrm{~kg}$ & 75 \\
\hline
\end{tabular}

\section{Rubber Compounding Materials}

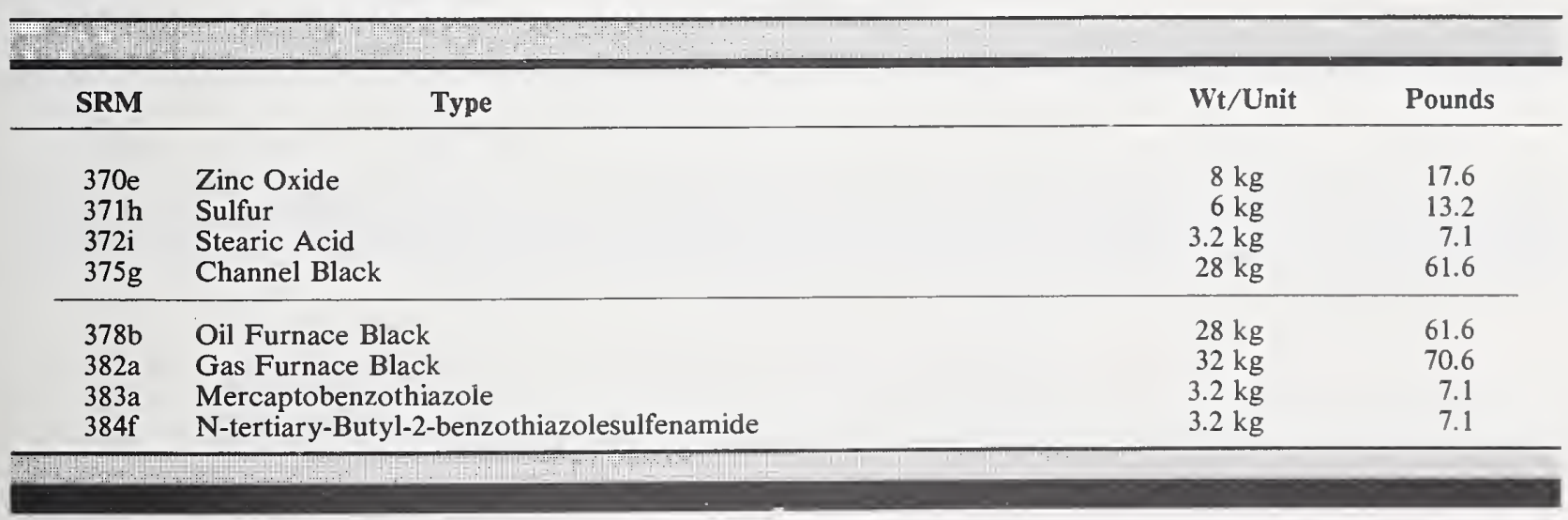




\section{Sizing}

\section{Particle Size}

SRM's 1003a, 1690, 1691, and 1960 can be used to calibrate various types of particle size measuring instruments including both light and electrical zone flow-through counters. SRM's 1004a, 1017a, 1018a, and 1019a are for calibrating test sieves.

\begin{tabular}{|c|c|c|c|c|}
\hline SRM & Type & Size $(\mu \mathrm{m})$ & Sieve No. & Wt/Unit \\
\hline $1003 \mathrm{a}$ & Glass Spheres & $8-58$ & - & $25 \mathrm{~g}$ \\
\hline $1004 \mathrm{a}$ & Glass Spheres & IN PREP & & \\
\hline $1017 \mathrm{a}$ & Glass Spheres & $100-310$ & $140-50$ & $84 \mathrm{~g}$ \\
\hline 1690 & Polystyrene Spheres ( $0.5 \%$ wt. concentration in water) & 0.895 & - & $5 \mathrm{~mL}$ vial \\
\hline 1691 & Polystyrene Spheres ( $0.5 \%$ wt. concentration in water) & 0.269 & - & $5 \mathrm{~mL}$ vial \\
\hline 1960 & Polystyrene Spheres ( $0.4 \%$ wt. concentration in water) & 9.89 & - & $5 \mathrm{~mL}$ vial \\
\hline 1961 & Polystyrene Spheres $(0.5 \%$ wt. concentration in water) & 29.64 & - & $5 \mathrm{~mL}$ vial \\
\hline 1962 & Polystyrene Spheres ( $0.5 \%$ wt. concentration in water) & 2.978 & - & $5 \mathrm{~mL}$ vial \\
\hline
\end{tabular}

\section{Cement Turbidimetric and Fineness}

This SRM is available to calibrate the Blaine fineness meter according to the latest issue of Federal Test Method Standard 158, Method 2101 or ASTM Designation C204; to calibrate the Wagner turbidimeter according to ASTM Designation C115; and to determine sieve residue according to ASTM Designation C430. Each set consists of twenty sealed vials, each containing approximately 10 grams of cement.

\begin{tabular}{cccccc}
\hline & & & & & \\
\hline SRM & Type & Properties Certified & Value & Unit \\
\hline \multirow{2}{*}{$114 \mathrm{n}$} & Portland Cement & $\begin{array}{c}\text { Residue on } 45 \mu \mathrm{m} \text {, electroformed sieve } \\
\text { wet method } \\
\text { Surface area (Wagner turbidimeter) } \\
\text { Surface area (Air-permeability) }\end{array}$ & $8.3 \%$ & $2020 \mathrm{~cm}^{2} \cdot \mathrm{g}^{-1}$ \\
& & $3460 \mathrm{~cm}^{2} \cdot \mathrm{g}^{-1}$ & Set of 20 vials \\
\hline
\end{tabular}




\section{Surface Area of Powders}

These materials are for calibrating and checking instruments used to determine the specific surface area of powders by BET. RM's 8005 through 8008 have been certified by the National Physical Laboratory, Teddington, U.K. (and meet the ISO definition for CRM's); RM's 8570, 8571, and 8572 are issued by NBS in cooperation with ASTM, but are not certified.

\begin{tabular}{|c|c|c|c|}
\hline $\mathbf{R M}$ & Type & Surface Area & Unit Size \\
\hline 8005 & Alpha Alumina (NPL\# M11-05/09) & $2.1 \mathrm{~m}^{2} / \mathrm{g}$ & $50 \mathrm{~g}$ \\
\hline 8006 & Alpha Alumina (NPL\# M11-06/10) & $0.3 \mathrm{~m}^{2} / \mathrm{g}$ & $50 \mathrm{~g}$ \\
\hline 8007 & Alpha Alumina (NPL\# M11-07/11) & $0.1 \mathrm{~m}^{2} / \mathrm{g}$ & $50 \mathrm{~g}$ \\
\hline 8008 & Alpha Alumina (NPL\# M11-08/12) & $0.8 \mathrm{~m}^{2} / \mathrm{g}$ & $50 \mathrm{~g}$ \\
\hline 8570 & Calcined Kaolin & $10.89 \mathrm{~m}^{2} / \mathrm{g}$ & $10 \mathrm{~g}$ \\
\hline 8571 & Alumina & $158.7 \mathrm{~m}^{2} / \mathrm{g}$ & $10 \mathrm{~g}$ \\
\hline 8572 & Silica-Alumina & $291.2 \mathrm{~m}^{2} / \mathrm{g}$ & $10 \mathrm{~g}$ \\
\hline
\end{tabular}

\section{PERFORMANCE STANDARDS}

\section{Socketed Ball Bar}

This SRM is for measuring the performance of coordinate measuring machines (CMM's) as per ASME Standard B89.1.12. It consists of a set of three precision balls pinned and cemented onto threaded shafts, one table-mount magnetic socket, one ram-mount magnetic socket, and 5 partially insulated extension tubes-50, $100,200,400$, and $800 \mathrm{~mm}$ long.

\begin{tabular}{|c|c|c|c|}
\hline SRM & Type & $\begin{array}{l}\text { Measuring Lengths } \\
\text { (50 } \mathrm{mm} \text { steps })\end{array}$ & Unit \\
\hline 2083 & Socketed Ball Bar & 100 to $1650 \mathrm{~mm}$ & Set \\
\hline
\end{tabular}

\section{Dye Penetrant Test Blocks}

These SRM's are for checking the performance of liquid dye penetrants and dye penetrant crack detection techniques. These test blocks have four synthetic cracks, approximately $0.2,0.5,1$, and $2 \mu \mathrm{m}$ wide.

\begin{tabular}{cccc}
\hline & & & \\
\hline SRM & Type & Surface & Unit Size \\
\hline 1850 & Penetrant Test Block & Bright Finish & $5 \mathrm{~cm} \mathrm{dia.,} 1 \mathrm{~cm} \mathrm{thick}$ \\
1851 & NDE Penetrant Test Block & Matte Finish & $5 \mathrm{~cm}$ dia., $1 \mathrm{~cm}$ thick \\
\hline
\end{tabular}




\section{Surface Roughness}

These SRM's are for calibrating stylus instruments that measure surface roughness. These electroless-nickel coated steel blocks have a sinusoidal roughness profile machined on the top surface.

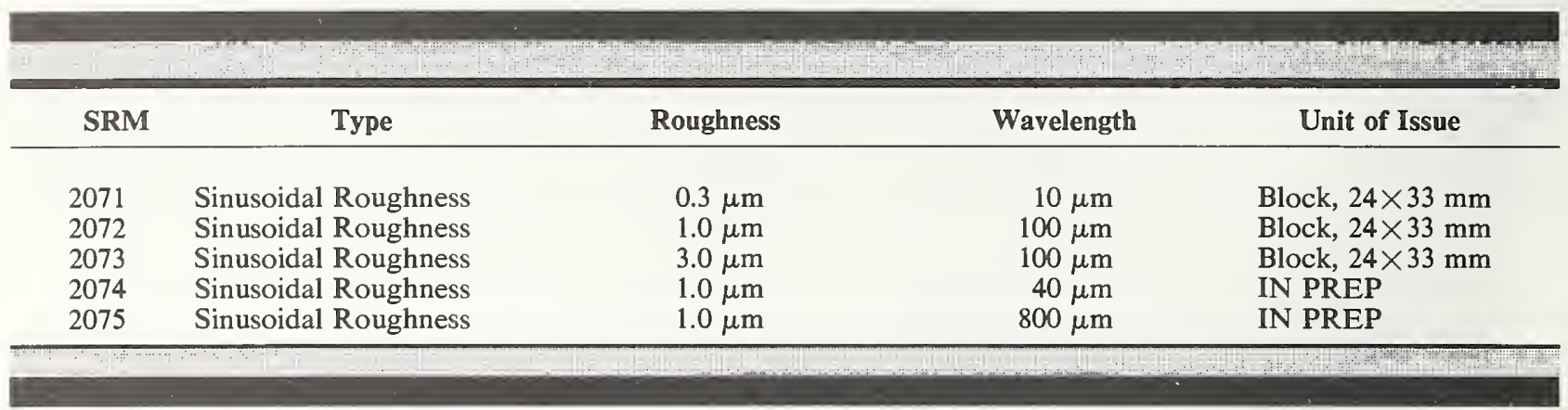

\section{Charpy V-Notch Test Blocks}

These SRM's are test specimens for standardizing the Charpy impact test and for certifying Charpy impact test machines. After the bars are broken in the user's laboratory, they are to be returned to NIST for examination and certification of the test machine.

\begin{tabular}{|c|c|c|c|}
\hline SRM & Type & Range & Unit of Issue \\
\hline 2092 & Charpy V-Notch Low Energy & $11-18 \mathrm{ft}-\mathrm{lb}$ & 5 bars \\
\hline 2096 & Charpy V-Notch High Energy & $65-80 \mathrm{ft}-\mathrm{lb}$ & 5 bars \\
\hline
\end{tabular}

\section{Artificial Flaw for Eddy Current NDE}

RM 8458 provides a flaw of known size and geometry that closely resembles an actual fatigue crack. It is intended to produce a response suitable for calibrating eddy current nondestructive evaluation (NDE) systems. The flaw size is $3.0 \pm 0.1 \mathrm{~mm}$ long by $1.0 \mathrm{~mm}$ deep in a $7 \times 7 \times 2 \mathrm{~cm}$ block of $7075-\mathrm{T} 651$ aluminum alloy, heat treated to the T6 temper. 


\section{Color}

These SRM's are available to illustrate a characteristic color for each of the ISCC-NBS color-name blocks in NBS Special Publication 440, COLOR: Universal Language and Dictionary of Names. SRM 2106 consists of 251 color chips on 18 constant-hue centroid color charts, and constitutes a supplement to SP 440 . The centroid colors represent a systematic sampling of the whole color solid. Note: The color chips were remeasured in 1984 and are issued with the new data as an addendum. This addendum is available upon request.

\begin{tabular}{lll}
\hline & & \\
\hline SRM & Type & Unit of Issue \\
\hline
\end{tabular}

\section{X-ray and Photographic}

SRM 1001 is a calibrated $x$-ray film step tablet of 17 steps that cover the optical density range from 0 to 4 ; it has a blue tint and emulsion on both sides. SRM 1008 is a calibrated photographic step tablet of 21 steps that cover the optical density range from 0 to 4 ; it has a black tint and emulsion on a single side.

SRM 1010a, Microcopy Resolution Test Charts, is used to test the resolving power of cameras or of whole microcopying systems. SRM 1010a consists of five charts printed photographically on paper, which have 26 high-contrast five-line patterns ranging in spatial frequency from one cycle per millimeter to 18 cycles per millimeter. Instructions for the use of the charts are supplied with each order.

\begin{tabular}{llc}
\hline & \multicolumn{1}{c}{ Type } & Unit \\
\hline SRM & & \\
1001 & X-ray Film Step Tablet (0-4) & 1 tablet, 17 steps \\
1008 & Photographic Step Tablet (0-4) & 1 tablet, 21 steps \\
$1010 a$ & Microcopy Resolution Test Chart & Set of 5 charts \\
\hline
\end{tabular}

\section{Magnetic Computer Storage Media}

These SRM's are for evaluating the performance of magnetic computer storage media and systems, and for maintaining control over their production. Each SRM is individually calibrated and certified.

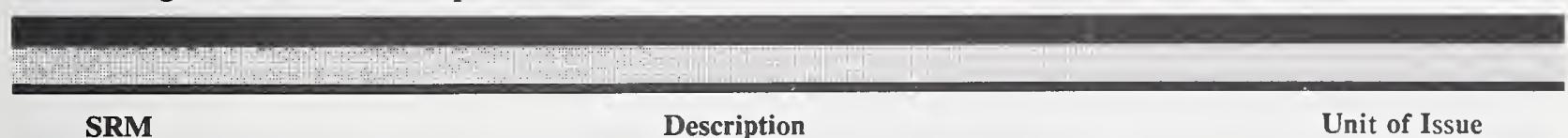

SRM

3200

6250
Secondary Standard Magnetic Tape-12.7 mm ( $1 / 2$ in) wide tape, certified for signal amplitude outputs relative to the NIST Standard Reference Amplitudes at 8,32 , and 126 flux transitions per millimeter $(200,800,3200$ flux transitions per inch).
Open Reel

Open Reel

Secondary Standard High Density Magnetic Tape-12.7 mm ( $1 / 2$ in) wide tape, certified for signal amplitude output relative to the NIST Standard Reference Amplitude at 356 flux transitions per millimeter (9042 flux transitions per inch). 


\section{Magnetic Computer Storage Media (Continued)}

\begin{tabular}{|c|c|c|}
\hline SRM & Description & Unit of Issue \\
\hline 1600 & $\begin{array}{l}\text { Secondary Standard Magnetic Tape Cassette-3.8 } \mathrm{mm}(0.15 \mathrm{in}) \text { wide tape, } \\
\text { certified for signal amplitude output relative to the NIST Standard Reference } \\
\text { Amplitude at } 63 \text { flux transitions per millimeter ( } 1600 \mathrm{flux} \text { transitions per inch). }\end{array}$ & Cassette \\
\hline 3216 & $\begin{array}{l}\text { Secondary Standard Magnetic Tape Cartridge- }-6.3 \mathrm{~mm}(1 / 4 \mathrm{in}) \text { wide tape, } \\
\text { certified for signal amplitude output relative to the NIST Standard Reference } \\
\text { Amplitude at } 126 \text { flux transitions per millimeter ( } 3200 \mathrm{flux} \text { transitions per inch). }\end{array}$ & Cartridge \\
\hline 3217 & $\begin{array}{l}\text { Secondary Standard High Density Magnetic Tape Cartridge- }-6.3 \mathrm{~mm}(1 / 4 \mathrm{in}) \\
\text { wide tape, certified for signal amplitude outputs relative to the NIST Standard } \\
\text { Reference Amplitudes at } 252 \text { and } 394 \text { flux transitions per millimeter ( } 6400 \text { and } \\
10,000 \text { flux transitions per inch). }\end{array}$ & Cartridge \\
\hline
\end{tabular}

These RM's are certified by the Physikalisch-Technische Bundesantalt (PTB), Federal Republic of Germany, for signal amplitude, overwrite, and resolution. The RM numbers correspond to the ISO standard number, and the materials conform to relevant ANSI, ISO, and ECMA standards for flexible disk cartridges.

\begin{tabular}{cccc|}
\hline & & & \\
\hline RM & Description & Unformatted Capacity & Unit/Size \\
\hline 6596 & Flexible Disk Cartridge & $125 \mathrm{~K}$ bytes & $130 \mathrm{~mm}(5.25 \mathrm{in})$ \\
9529 & Flexible Disk Cartridge & $2000 \mathrm{~K}$ bytes & $90 \mathrm{~mm}(3.5 \mathrm{in})$ \\
\hline
\end{tabular}

\section{Centerline Drawings for Optical Character Recognition Style-B Characters}

This SRM is an exact copy of the centerline drawings that uniquely define each printed character shape and size used in constant strokewidth Style B Size I Optical Character Recognition (OCR-B) applications in accordance with one or more of the following standards: American National Standard X3.49-1975 (R 1982), Character Set for Optical Character Recognition (OCR-B); Federal Information Processing Standards Publication (OCR), European Computer Manufacturers Association Standard ECMA-11 for the Alphanumeric Character Set OCR-B for Optical Recognition, 3rd Edition, 1976 and International Standard ISO 1073/II-1976, Alphanumeric Character Sets for Optical Recognition Part II: Character Set OCR-B.

This Standard Reference Material contains information on the shape, size, strokewidth, and position relative to the base line of the OCR-B characters.

\begin{tabular}{ccccc}
\hline & & & & \\
\hline SRM & Characters & Sheets & Size & Sheet Size \\
\hline 1901 & 118 & 118 & OCR-B I & $32 \times 44 \times 0.01 \mathrm{~cm}$ \\
\hline
\end{tabular}




\section{NIST Time Software}

The NIST automated time service is a telephone time service designed to provide computer with telephone access to NIST time at occurrences approaching one millisecond ( 0.001 second). Features of the service include automated compensation for telephone-line delay, advanced alert for changes to and from daylight savings time and advanced notice of insertion of leap seconds. The ASC11-character time code should operate with standard modems and most computer systems. While the system can be used to set up computer time-of-day, simple hardware can be developed to set non-computer clock system.

\begin{tabular}{ccc}
\hline SRM & Description & Unit of Issue \\
\hline 8101 & Automated Computer Time Service (Acts) & Diskette \\
\hline
\end{tabular}

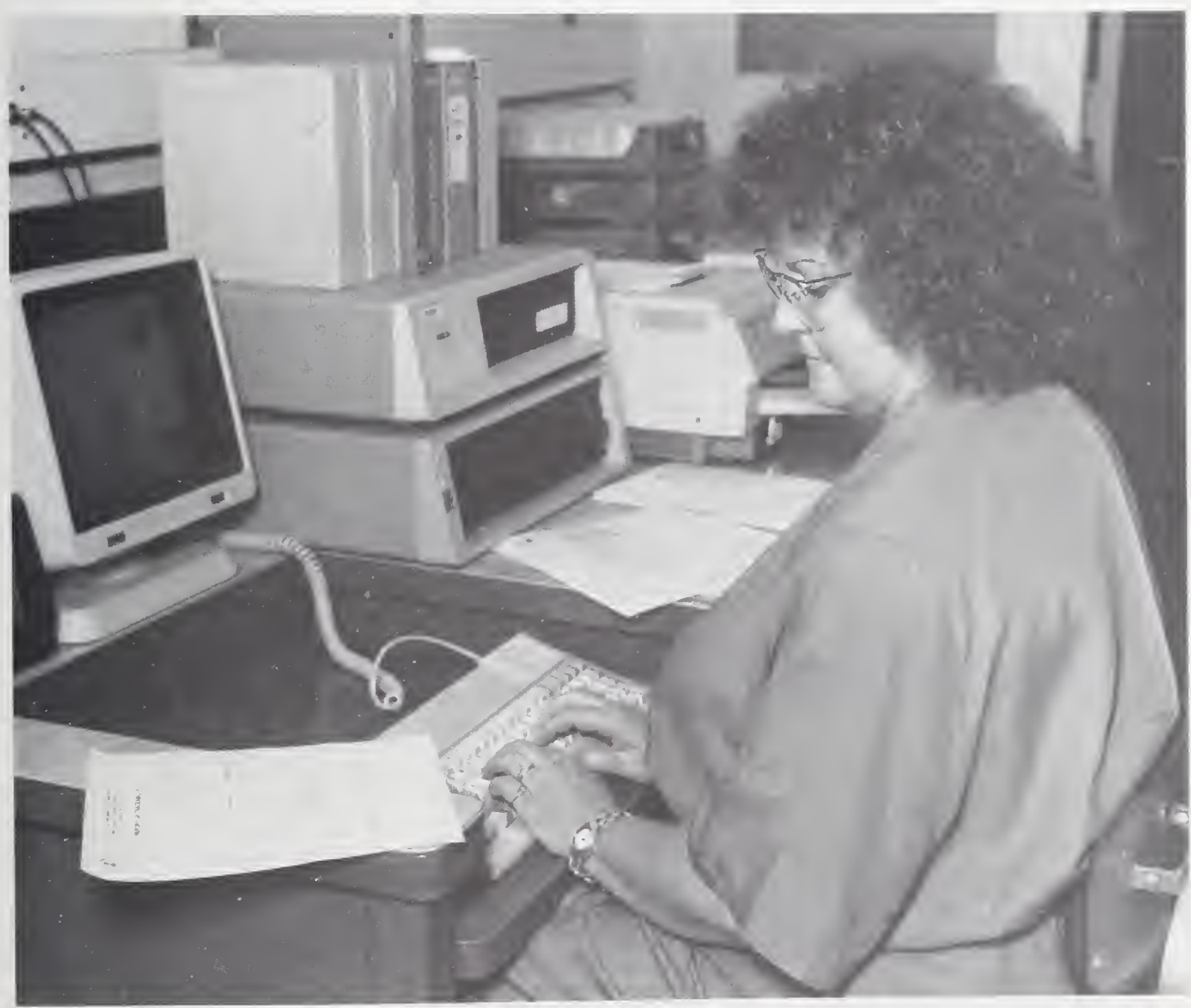

Cassandra Beck responds to one of the many inquiries or orders she handles for radioactivity SRM's. 


\section{FIRE RESEARCH}

\section{Surface Flammability}

SRM 1002d, Hardboard Sheet, is issued for checking the operation of radiant panel test equipment in accordance with the procedures outlined in ASTM Standard E162-78.

$\begin{array}{cllll} & & & \\ \text { SRM } & \text { Type } & \text { Certification } & \text { Unit of Issue }\end{array}$

1002d Hardboard Sheet
Flame Spread Index, I=153

Heat Evolution Factor, $Q=36.5$
Set of $4: 6 \times 18 \times 1 / 4$ inch

\section{Smoke Density Chamber}

These SRM's are certified for maximum specific optical density and are issued for performing operational checks of smoke density chambers.

\begin{tabular}{|c|c|c|c|}
\hline SRM & Туре & $\begin{array}{c}\text { Maximum Specific Optical } \\
\text { Density }\end{array}$ & Unit of Issue \\
\hline $1006 \mathrm{c}$ & Non-flaming Exposure Condition ( $\alpha$-cellulose) & $\operatorname{Dm}($ corr. $)=178$ & 6 sheets \\
\hline $1007 \mathrm{a}$ & Flaming Exposure Condition (plastic) & $\mathrm{Dm}($ corr. $)=421$ to 493 & 3 sheets \\
\hline
\end{tabular}

\section{Flooring Radiant Panel}

This SRM consists of three sheets of kraft paperboard. It is for checking the operation of flooring radiant panel test apparatus used to measure critical radiant flux as per ASTM E648.

\begin{tabular}{|c|c|c|c|}
\hline SRM & Type & Critical Radiant Flux & Unit Size (cm) \\
\hline 1012 & Flooring Radiant Panel & $0.36 \mathrm{~W} / \mathrm{cm}^{2}$ & $104.1 \times 25.4 \times 0.305$ \\
\hline
\end{tabular}

\section{Tape Adhesion Testing}

This material is intended as a uniform source of linerboard for use under ASTM Designation D2860, Procedure A: Adhesion of Pressure Sensitive Tape to Fiberboard at 90 Degree Angle and Constant Stress.

\begin{tabular}{lll} 
& & \\
\hline SRM & Type
\end{tabular}




\section{Additional Information}

\section{NIST SPECIAL PUBLICATIONS IN THE 260 SERIES}

Barber, S. L., Hines, J. K., editors, NIST Standard Reference Materials Catalog (1990-91 edition), NIST Spec. Publ. 260 (January 1990)SN003-003-00000-0.

Michaelis, R. E., and Wyman, L. L., Standard Reference Materials: Preparation of White Cast Iron Spectrochemical Standards, NBS Misc. Publ. 260-1 (June 1964). COM74-11061**

Michaelis, R. E., Wyman, L. L., and Flitsch, R., Standard Reference Materials: Preparation of NBS Copper-Base Spectrochemical Standards, NBS Misc. Publ. 260-2 (October 1964). COM74-11063**

Michaelis, R. E., Yakowitz, H., and Moore, G. A., Standard Reference Materials: Metallographic Characterization of an NBS Spectrometric Low-Alloy Steel Standard, NBS Misc. Publ. 260-3 (October 1964). COM74-11060**

Alvarez, R., and Flitsch, R., Standard Reference Materials: Accuracy of Solution X-Ray Spectrometric Analysis of Copper-Base Alloys, NBS Misc. Publ. 260-5 (March 1965). PB168068**

Shultz, J. I., Standard Reference Materials: Methods for the Chemical Analysis of White Cast Iron Standards, NBS Misc. Publ. 260-6 (July 1965). COM74-11068**

Bell, R. K., Standard Reference Materials: Methods for the Chemical Analysis of NBS Copper-Base Spectrochemical Standards, NBS Misc. Publ. 260-7 (October 1965). COM74-11067**

* Send order with remittance to Superintendent of Documents, US Government Printing Office, Washington, DC 20402. Remittance from foreign countries should include an additional one-fourth of the purchase price for postage.

** May be ordered from: National Technical Information Services (NTIS), Springfield, Virginia 22161.
Richmond, M.S., Standard Reference Materials: Analysis of Uranium Concentrates at the National Bureau of Standards, NBS Misc. Publ. 260-8 (December 1965). COM74-11066**

Anspach, S. C., Cavallo, L. M., Garfinkel, S. B., Hutchinson, J. M. R., and Smith, C. N., Standard Reference Materials: Half Lives of Materiass Used in the Preparation of Standard Reference Materials of Nineteen Radioactive Nuclides Issued by the National Bureau of Standards, NBS Misc. Publ. 260-9 (November 1965). COM74-11065**

Yakowitz, H., Vieth, D. L., Heinrich, K. F. J., and Michaelis, R. E., Standard Reference Materials: Homogeneity Characterization of NBS Spectrometric Standards II: Cartridge Brass and Low-Alloy Steel, NBS Misc. Publ. 260-10 (December 1965). COM74-11064**

Yakowitz, H., Michaelis, R. E., and Vieth, D. L., Standard Reference Materials: Homogeneity

Characterization of NBS Spectrometric Standards IV: Preparation and Microprobe Characterization of W-20\% Mo

Alloy Fabricated by Powder Metallurgical Methods, NBS Spec. Publ. 260-16 (January 1969). COM74-11062**

Paule, R. C., and Mandel, J., Standard Reference Materials: Analysis of Interlaboratory Measurements on the Vapor Pressure of Gold (Certification of Standard Reference Material 745), NBS Spec. Publ. 260-19 (January 1970). PB190071**

Paule, R. C., and Mandel, J., Standard Reference Materials: Analysis of Interlaboratory Measurements on the Vapor Pressures of Cadmium and Silver, NBS Spec. Publ. 260-21 (January 1971). COM74-11359**
Yakowitz, H., Fiori, C. E., and Michaelis, R. E., Standard Reference Materials: Homogeneity Characterization of $\mathrm{Fe}-3 \mathrm{Si}$ Alloy, NBS Spec. Publ. 260-22 (February 1971). COM74-11357**

Napolitano, A., and Hawkins, E. G., Standard Reference Materials: Viscosity of a Standard Borosilicate Glass, NBS Spec. Publ. 260-23 (December 1970). COM71-00157**

Sappenfield, K. M., Marinenko, G., and Hague, J. L., Standard Reference Materials: Comparison of Redox Standards, NBS Spec. Publ. 260-24 (January 1972). COM72-50058**

Hicho, G. E., Yakowitz, H., Rasberry, S. D., and Michaelis, R. E., Standard Reference Materials: A Standard Reference Material Containing Nominally Four Percent Austenite, NBS Spec. Publ. 260-25 (February 1971). COM74-11356**

Martin, J. F., Standard Reference Materials: National Bureau of Standards-US Steel Corporation Joint Program for Determining Oxygen and Nitrogen in Steel, NBS Spec. Publ. 260-26 (February 1971). 85 cents* PB 81176620

Garner, E. L., Machlan, L. A., and Shields, W. R., Standard Reference Materials: Uranium Isotopic Standard Reference Materials, NBS Spec. Publ. 260-27 (April 1971). COM74$11358 * *$

Heinrich, K. F. J., Myklebust, R. L., Rasberry, S. D., and Michaelis, R. E., Standard Reference Materials: Preparation and Evaluation of SRM's 481 and 482 Gold-Silver and GoldCopper Alloys for Microanalysis, NBS Spec. Publ. 260-28 (August 1971). COM71-50365**

Geller, S. B., Standard Reference Materials: Calibration of NBS Secondary Standard Magnetic Tape (Computer Amplitude Reference) Using the Reference Tape Amplitude Measurement "Process A-Model 2," NBS Spec. Publ. 260-29 (June 1971). COM71-50282** 
Gorozhanina, R. S., Freedman, A. Y., and Shaievitch, A. B. (translated by M. C. Selby), Standard Reference Materials: Standard Samples Issued in the USSR (A Translation from the Russian), NBS Spec. Publ. 260-30 (June 1971). COM71-50283**

Hust, J. G., and Sparks, L. L., Standard Reference Materials: Thermal Conductivity of Electrolytic Iron SRM 734 from 4 to $300 \mathrm{~K}$, NBS Spec. Publ. 260-31 (November 1971). COM71-50563**

Mavrodineanu, R., and Lazar, J. W., Standard Reference Materials: Standard Quartz Cuvettes for High Accuracy Spectrophotometry, NBS Spec. Publ. 260-32 (December 1973). COM74-50018**

Wagner, H. L., Standard Reference Materials: Comparison of Original and Supplemental SRM 705, Narrow Molecular Weight Distribution Polystyrene, NBS Spec. Publ. 260-33 (May 1972). COM72-50526**

Sparks, L. L., and Hust, J. G., Standard Reference Materials: Thermal Conductivity of Austenitic Stainless Steel, SRM 735 from 5 to $280 \mathrm{~K}$, NBS Spec. Publ. 260-35 (April 1972). COM72-50368**

Cali, J. P., Mandel, J., Moore, L. J., and Young, D. S., Standard Reference Materials: A Referee Method for the Determination of Calcium in Serum, NBS SRM 915, NBS Spec. Publ. 260-36 (May 1972). COM72-50527**

Shultz, J. I., Bell., R. K., Rains, T. C., and Menis, O., Standard Reference Materials: Methods of Analysis of NBS Clay Standards, NBS Spec. Publ. 260-37 (June 1972). COM7250692**

Richmond, J. C., and Hsia, J. J., Standard Reference Materials: Preparation and Calibration of Standards of Spectral Specular Reflectance, NBS Spec. Publ. 260-38 (May 1972). COM72-50528**

Clark, A. F., Denson, V.A., Hust, J. G., and Powell, R. L., Standard Reference Materials: The Eddy Current Decay Method for Resistivity Characterization of HighPurity Metals, NBS Spec. Publ. 26039 (May 1972). COM72-50529**

McAdie, H. G., Garn, P.D., and Menis, O., Standard Reference Materials: Selection of Thermal Analysis Temperature Standards Through a Cooperative Study (SRM 758, 759, 760), NBS Spec. Publ. 26040 (August 1972). COM72-50776**
Wagner, H. L., and Verdier, P. H., eds., Standard Reference Materials: The Characterization of Linear Polyethylene, SRM 1475, NBS Spec. Publ. 260-42 (September 1972). COM72-50944**

Yakowitz, H., Ruff, A. W., and Michaelis, R. E., Standard Reference Materials: Preparation and Homogeneity Characterization of an Austenitic Iron-Chromium-Nickel Alloy, NBS Spec. Publ. 260-43 (November 1972). COM73-50760**

Schooley, J. F., Soulen, R. J., Jr., and Evans, G. A., Jr., Standard Reference Materials: Preparation and Use of Superconductive Fixed Point Devices, SRM 767, NBS Spec. Publ. 260-44 (December 1972). COM73$50037 * *$

Greifer, B., Maienthal, E. J., Rains, T. C., and Rasberry, S. D., Standard Reference Materials: Powdered Lead-Based Paint, SRM 1579, NBS Spec. Publ. 260-45 (March 1973). COM73-50226**

Hust, J. G., and Giarratano, P. J., Standard Reference Materials: Thermal Conductivity and Electrical Resistivity Standard Reference Materials: Austenitic Stainless Steel, SRM's 735 and 798, from 4 to 1200 K, NBS Spec. Publ. 260-46 (March 1975). COM75-10339**

Hust, J. G., Standard Reference Materials: Electrical Resistivity of Electrolytic Iron, SRM 797, and Austenitic Stainless Steel, SRM 798, from 5 to $280 \mathrm{~K}$, NBS Spec. Publ. 260-47 (February 1974). COM74$50176 * *$

Mangum, B. W., and Wise, J. A., Standard Reference Materials: Description and Use of Precision Thermometers for the Clinical Laboratory, SRM 933 and SRM 934, NBS Spec. Publ. 260-48 (May 1974). COM74-50533**

Carpenter, B. S., and Reimer, G. M., Standard Reference Materials: Calibrated Glass Standards for Fission Track Use, NBS Spec. Publ. 260-49 (November 1974). COM74$51185^{* *}$

Hust, J. G., and Giarratano, P. J., Standard Reference Materials: Thermal Conductivity and Electrical Resistivity Standard Reference Materials: Electrolytic Iron, SRM's 734 and 797 from 4 to $1000 \mathrm{~K}$, NBS Spec. Publ. 260-50 (June 1975). COM75-10698**
Mavrodineanu, R., and Baldwin, J. R., Standard Reference Materials: Glass Filters as a Standard Reference Material for SpectrophotometrySelection, Preparation, Certification, Use-SRM 930, NBS Spec. Publ. 260-51 (November 1975). COM75$10339 * *$

Hust, J. G., and Giarratano, P. J., Standard Reference Materials: Thermal Conductivity and Electrical Resistivity Standard Reference Materials 730 and 799, from 4 to 3000 K, NBS Spec. Publ. 260-52 (September 1975). COM75-11193**

Durst, R. A., Standard Reference Materials: Standardization of $\mathrm{pH}$ Measurements, NBS Spec. Publ. 26053 (December 1975, Revised). PB248127**

Burke, R. W., and Mavrodineanu, R., Standard Reference Materials: Certification and Use of Acidic Potassium Dichromate Solutions as an Ultraviolet Absorbance Standard, NBS Spec. Publ. 260-54 (August 1977). PB272168**

Ditmars, D. A., Cezairliyan, A., Ishihara, S., and Douglas, T. B., Standard Reference Materials: Enthalpy and Heat Capacity; Molybdenum SRM 781, from 273 to 2800 K, NBS Spec. Publ. 260-55 (September 1977). PB272127**

Powell, R. L., Sparks, L. L., and Hust, J. G., Standard Reference Materials: Standard Thermocouple Materials, Pt 67: SRM 1967, NBS Spec. Publ. 260-56 (February 1978). PB277172**

Barnes, J. D., and Martin, G. M., Standard Reference Materials: Polyester Film for Oxygen Gas Transmission Measurements SRM 1470, NBS Spec. Publ. 260-58 (June 1979). PB297098**

Velapoldi, R. A., Paule, R. C., Schaffer, R., Mandel, J., and Moody, J. R., Standard Reference Materials: A Reference Method for the Determination of Sodium in Serum, NBS Spec. Publ. 260-60 (August 1978). PB286944**

Verdier, P. H., and Wagner, H. L., Standard Reference Materials: The Characterization of Linear Polyethylene (SRM 1482, 1483, 1484), NBS Spec. Publ. 260-61 (December 1978). PB289899** 
Soulen, R. J., and Dove, R. B., Standard Reference Materials: Temperature Reference Standard for Use Below 0.5 K (SRM 768), NBS Spec. Publ. 260-62 (April 1979). PB294245**

Velapoldi, R. A., Paule, R. C., Schaffer, R., Mandel, J., Machlan, L. A., and Gramlich, J. W., Standard Reference Materials: A Reference Method for the Determination of Potassium in Serum, NBS Spec. Publ. 260-63 (May 1979). PB297207**

Velapoldi, R. A., and Mielenz, K. D., Standard Reference Materials: A Fluorescence Standard Reference Material Quinine Sulfate Dihydrate (SRM 936), NBS Spec. Publ. 260-64 (January 1980). PB-80-132046**

Marinenko, R. B., Heinrich, K. F. J., and Ruegg, F. C., Standard Reference Materials: Micro-Homogeneity Studies of NBS Standard Reference Materials, NBS Research Materials, and Other Related Samples, NBS Spec. Publ. 260-65 (September 1979). PB300461**

Venable, W. H., Jr., and Eckerle, K. L., Standard Reference Materials: Didymium Glass Filters for Calibrating the Wavelength Scale of Spectrophotometers-SRM 2009, 2010, 2013 and 2014, NBS Spec. Publ. 260-66 (October 1979). PB-80$104961 * *$

Velapoldi, R. A., Paule, R. C., Schaffer, R., Mandel, J., Murphy, T. J., and Gramlich, J. W., Standard Reference Materials: A Reference Method for the Determination of Chloride in Serum, NBS Spec. Publ. 260-67 (November 1979). PB-80$110117^{* *}$

Mavrodineanu, R., and Baldwin, J. R., Standard Reference Materials: MetalOn-Quartz Filters as a Standard

Reference Material for

Spectrophotometry-SRM 2031, NBS Spec. Publ. 260-68 (April 1980). PB80-197486**

Velapoldi, R. A., Paule, R. C., Schaffer, R., Mandel, J., Machlan, L. A., Garner, E. L., and Rains, T. C., Standard Reference Materials: A Reference Method for the Determination of Lithium in Serum, NBS Spec. Publ. 260-69 (July 1980). PB-80-20917**

Marinenko, R. B., Biancaniello, F., Boyer, P. A., Ruff, A. W., and DeRobertis, L., Standard Reference Materials: Preparation and Characterization of an IronChromium-Nickel Alloy for Microanalysis, NBS Spec. Publ. 26070 (May 1981). PB-84-165349**
Seward, R. W., and Mavrodineanu, R., Standard Reference Materials:

Summary of the Clinical Laboratory

Standards Issued by the National

Bureau of Standards, NBS Spec.

Publ. 260-71 (November 1981). PB82-135161**

Reeder, D. J., Coxon, B., Enagonio, D., Christensen, R. G., Schaffer, R., Howell, B. F., Paule, R. C., and Mandel, J., Standard Reference Materials: SRM 900, Antiepilepsy Drug Level Assay Standard, NBS Spec. Publ. 260-72 (June 1981). PB81-220758**

Interrante, C. G., and Hicho, G. E., Standard Reference Materials: A Standard Reference Material Containing Nominally Fifteen Percent Austenite (SRM 486), NBS Spec. Publ. 260-73 (January 1982). PB-82-215559**

Marinenko, R. B., Standard

Reference Materials: Preparation and Characterization of $\mathrm{K}-411$ and $\mathrm{K}-414$ Mineral Glasses for Microanalysis: SRM 470, NBS Spec. Publ. 260-74 (April 1982). PB-82-221300**

Weidner, V. R., and Hsia, J. J., Standard Reference Materials: Preparation and Calibration of First Surface Aluminum Mirror Specular Reflectance Standards (SRM 2003a), NBS Spec. Publ. 260-75 (May 1982). PB-82-221367**

Hicho, G. E., and Eaton, E. E., Standard Reference Materials: A Standard Reference Material Containing Nominally Five Percent Austenite (SRM 485a), NBS Spec. Publ. 260-76 (August 1982). PB-83$115568^{* *}$

Furukawa, G. T., Riddle, J. L., Bigge, W. G., and Pfieffer, E. R., Standard Reference Materials: Application of Some Metal SRM's as Thermometric Fixed Points, NBS Spec. Publ. 26077 (August 1982). PB-83-117325**

Hicho, G. E., and Eaton, E. E., Standard Reference Materials: Standard Reference Material Containing Nominally Thirty Percent Austenite (SRM 487), NBS Spec. Publ. 260-78 (September 1982). PB83-115576**

Richmond, J. C., Hsia, J. J., Weidner, V. R., and Wilmering, D. B., Standard Reference Materials: Second Surface Mirror Standards of Specular Spectral Reflectance (SRM's 2023, 2024, 2025), NBS Spec. Publ. 260-79 (October 1982). PB-84-203447**
Schaffer, R., Mandel, J., Sun, 'T., Cohen, A., and Hertz, H. S., Standard Reference Materials: Evaluation by an ID/MS Method of the AACC Reference Method for Scrum Glucose, NBS Spec. Publ. 260-80 (October 1982). PB-84-216894**

Burke, R. W., and Mavrodineanu, R., Standard Reference Materials:

Accuracy in Analytical

Spectrophotometry, NBS Spec. Publ. 260-81 (April 1983). PB-83-214536**

Weidner, V. R., Standard Reference Materials: White Opal Glass Diffuse Spectral Reflectance Standards for the Visible Spectrum (SRM's 2015 and 2016), NBS Spec. Publ. 260-82 (April 1983). PB-83-220723**

Bowers, G. N., Jr., Alvarez, R., Cali, J. P., Eberhardt, K. R., Reeder, D. J., Schaffer, R., and Uriano, G. A., Standard Reference Materials: The Measurement of the Catalytic (Activity) Concentration of Seven Enzymes in NBS Human Serum SRM 909, NBS Spec. Publ. 260-83 (June 1983). PB-83-239509**

Gills, T. E., Seward, R. W., Collins, R. J., and Webster, W. C., Standard Reference Materials: Sampling, Materials Handling, Processing, and Packaging of NBS Sulfur in Coal Standard Reference Materials 2682, 2683, 2684, and 2685, NBS Spec. Publ. 260-84 (August 1983). PB-84$109552 * *$

Swyt, D. A., Standard Reference Materials: A Look at Techniques for the Dimensional Calibration of Standard Microscopic Particles, NBS Spec. Publ. 260-85 (September 1983). PB-84-112648**

Hicho, G. E., and Eaton, E. E., Standard Reference Materials: A Standard Reference Material Containing Two and One-Half Percent Austenite, SRM 488, NBS Spec. Publ. 260-86 (December 1983). PB-84-143296**

Mangum, B. W., Standard Reference Materials: SRM 1969: Rubidium Triple-Point-A Temperature Reference Standard Near $39.30^{\circ} \mathrm{C}$. NBS Spec. Publ. 260-87 (December 1983). PB-84-149996

Hust, J. G., Standard Reference Materials: A Fine-Grained, Isotropic Graphite for Use as NBS

Thermophysical Property RM's from 5 to 2500 K. NBS Spec. Publ. 260-89 (September 1984). PB-85-1128\$6** 
Hust, J. G., and Lankford, A. B., Standard Reference Materials: Update of Thermal Conductivity and Electrical Resistivity of Electrolytic Iron, Tungsten, and Stainless Steel, NBS Spec. Publ. 260-90 (September 1984). PB-85-115814**

Goodrich, L. F., Vecchia D. F., Pittman, E. S., Ekin, J. W. and Clark, A. F., Standard Reference Materials: Critical Current Measurements on an NbTi Superconducting Wire Standard Reference Material, NBS Spec. Publ. 260-91 (September 1984). SN003-003-02614-0*

Carpenter, B. S., Standard Reference Materials: Calibrated Glass Standards for Fission Track Use (Supplement to NBS Spec. Publ. 260-49). NBS Spec. Publ. 260-92 (September 1984). PB$85-113025$ **

\section{Ehrstein, J., Standard Reference}

Materials: Preparation and

Certification of Standard Reference

Materials for Calibration of

Spreading Resistance Probes, NBS

Spec. Publ. 260-93 (January 1985).

PB-85-177921**

Gills, T. E., Koch, W. F., Stolz, J. W. Kelly, W. R., Paulsen, P. J., Colbert, J. C., Kirklin, D. R., Pei, P. T. S., Weeks, S., Lindstrom, R. M., Fleming, R. F., Greenberg, R. R., and Paule, R. C., Standard Reference Materials: Methods and Procedures Used at the National Bureau of Standards to Certify Sulfur in Coal SRM's for Sulfur Content, Calorific Value, Ash Content, NBS Spec. Publ. 260-94 (December 1984). PB-85-165900**

Mulholland, G. W., Hartman, A. W., Hembree, G. G., Marx, E., and Lettieri, T. R., Standard Reference Materials: Development of a $1 \mu \mathrm{m}$ Diameter Particle Size Standard, SRM 1690, NBS Spec. Publ. 260-95 (May 1985). SN003-003-02665-4*
Carpenter, B. S., Gramlich, J. W., Greenberg, R. R., Machlan, L. A., DeBievre, P., Eschbach, H. L., Meyer, H., Van Andenhove, J., Connelly, V. E., Trahey, N. M., and Zook, A. C., Standard Reference Materials: Uranium-235 Isotopic Abundance Standard Reference Materials for Gamma Spectrometry Measurements, NBS Spec. Publ. 26096 (September 1986). PB-87$108544 * *$

Mavrodineanu, R., and Gills, T. E., Standard Reference Materials: Summary of the Coal, Ore, Mineral, Rock, and Refractory Standards Issued by the National Bureau of Standards, NBS Spec. Publ. 260-97 (September 1985). SN003-003-026883*

Hust, J. G., Standard Reference Materials: Glass Fiberboard SRM for Thermal Resistance, NBS Spec. Publ. 260-98 (August 1985). SN003-00302674-3

Callanan, J. E., Sullivan, S. A., and Vecchia, D. F., Standard Reference Materials: Feasibility Study for the Development of Standards Using Differential Scanning Calorimetry, NBS Spec. Publ. 260-99 (August 1985). SN003-003-02675-1*

Taylor, J. K., Standard Reference Materials: Handbook for SRM Users, NBS Spec. Publ. 260-100 (September 1985). PB-86-110897**

Mangum, B. W., Standard Reference Materials: SRM 1970, Succinonitrile Triple-Point Standard: A

Temperature Reference Standard Near $58.08^{\circ} \mathrm{C}$, NBS Spec. Publ. 260101 (March 1986). SN003-00302722-7*

Weidner, V. R., Mavrodineau, R., Mielenz, K. D., Velapoldi, R. A., Eckerle, K. L., and Adams, B., Standard Reference Materials: Holmium Oxide Solution Wavelength Standard from $240-650 \mathrm{~nm}$, SRM 2034, NBS Spec. Publ. 260-102 (July 1986). PB-86-245727**

Hust, J. G., Standard Reference Materials: Glass Fiberblanket SRM for Thermal Resistance, NBS Spec. Publ. 260-103 (September 1985). SN003-003-02687-5*
Mavrodineanu, R., and Alvarez, R., Standard Reference Materials: Summary of the Biological and Botanical Standards Issued by the National Bureau of Standards, NBS Spec. Publ. 260-104 (November 1985). SN003-003-02704-9*

Mavrodineanu, R., and Rasberry, S. D., Standard Reference Materials: Summary of the Environmental Research Analysis, and Control Standards Issued by the National Bureau of Standards, NBS Spec. Publ. 260-105 (March 1986). SN003003-02725-1*

Koch, W. F., ed., Standard Reference Materials: Methods and Procedures Used at the National Bureau of Standards to Prepare, Analyze, and Certify SRM 2694, Simulated Rainwater, and Recommendations for Use, NBS Spec. Publ. 260-106 (July 1986). PB-86-247483**

Hartman, A. W., McKenzie, R. L. Standard Reference Materials: SRM 1965, Microsphere Slide (10 $\mu \mathrm{m}$ Polystyrene Spheres), NIST Spec. Publ. 260-107 (November 1988)

Mavrodineanu, R., and Gills, T. E., Standard Reference Materials: Summary of Gas Cylinder and Permeation Tube Standard Reference Materials Issued by the National Bureau of Standards, NBS Spec. Publ. 260-108 (May 1987).

Candela, G. A., Chandler-Horowitz, D., Novotny, D. B., Marchiando, J. F., and Belzer, B. J., Standard Reference Materials: Preparation and Certification of an Ellipsometrically Derived Thickness and Refractive Index Standard of a Silicon Dioxide Film (SRM 2530), NIST Spec. Publ. 260-109 (October 1988).

Kirby, R. K., and Kanare, H. M., Standard Reference Materials: Portland Cement Chemical Composition Standards (Blending, Packaging, and Testing), NBS Spec. Publ. 260-110 (February 1988).

Gladney, E. S., O'Malley, B. T. Roelandts, I., Gills, T. E. Standard Reference Materials: Compilation of Elemental Concentration Data for NBS Clinical, Biological, Geological, and Environmental, NBS Spec. Publ. 260-111 (November 1987) 


\section{NIST Calibration Service Contacts}

\section{Measurement Area}

General Information

Dimensional Measurements

Angular

API Plug and Ring Gages

End Standards

Gage Blocks

Hydrometers

Length and Diameter; Step Gages

Line Standards

Measuring Wires

Micrometers; Penetration Needles

Optical Reference Planes

Plain Conical; Threaded Plug and Ring Gages

Roundness

Sieves

Spherical Diameter; Plug Gages; Ring Gages

Surface Texture

Surveying Rods and Tapes; Two Dimensional Gages

Volume and Density

Electromagnetic Measurements

AC-DC Voltage/Current Converters (to $1 \mathrm{MHz}$ )

AC Resistors

Capacitance Dividers

Coaxial/Waveguide Terminations Reflection Coefficients

Current Transformers

Data Converters

DC Resistance

DC Voltage

Electromagnetic Field-Strength Parameters

HF Capacitance/Inductance

High Frequency Resistors

High Frequency Voltage

Inductive Dividers

LF AC Voltmeters and Sources

LF Capacitance/Inductance

LF Power/Energy

Microwave Antenna Parameter

Mixed Dividers

N-Port Scattering

Noise Temperature

Phase Angle Meters

Power-Frequency Capacitors

Pulse Waveform

Q Standards

Resistive Dividers

RF-DC Voltage/Current Converter $(100 \mathrm{~Hz}-1 \mathrm{GHz})$

RF/Microwave Attenuators

RF/Microwave Phase Shifters

RF/Microwave Power Meters

VHF Omnidirectional Range

Voltage Transformers

Ionizing Radiation Measurements

Dosimetry of X rays, Gamma Rays and Electrons

High-Dose Dosimetry

Neutron Sources and Dosimeters

Radioactivity Sources
Joe D. Simmons or

Measurement Services Staff

Yun H. Wang

Edgar G. Erber

Theodore D. Doiron

John R. Stoup

John F. Houser

Theodore D. Doiron

William B. Penzes

David Stieren

Edgar G. Erber

Jay H. Zimmerman

Edgar G. Erber

Yun $\mathrm{H}$. Wang

Theodore D. Doiron

Yun $\mathrm{H}$. Wang

Arie Hartman

Ronald G. Hartsock

John F. Houser

Joseph R. Kinard

T. Michael Souders

Robert E. Hebner, Jr.

John R. Juroshek

John D. Ramboz

T. Michael Souders

Ronald F. Dziuba

June E. Sims

Galen H. Koepke

George M. Free

George M. Free

Robert E. Hebner, Jr.

Bruce F. Field

Nile M. Oldham

Bruce F. Field

Andrew J. Secula

Allen C. Newell

Edward F. Kelley

Ronald A. Ginley

Sunchana Perera

Raymond S. Turgel

William E. Anderson

William L. Gans

George M. Free

Martin Misakian

Gregorio Rebuldela

John R. Juroshek

John L. Workman

Ronald A. Ginley

Neil T. Larsen

William E. Anderson

Bert M. Coursey

William L. McLaughlin

E. Dale McGarry

Jacqueline M. Calhoun
(301) 975-3468

(301) 975-3468

(301) 975-3468

(301) 975-3468

(301) 975-5956

(301) 975-3468

(301) 975-3468

(301) 975-3468

(301) 975-3468

(301) 975-3468

(301) 975-3468

(301) 975-3468

(301) 975-3468

(301) 975-3468

(301) 975-3475

(301) 975-3465

(301) 975-5956

(301) 975-4250

(301) 975-2406

(301) 975-2403

(303) 497-5362

(301) 975-2434

(301) 975-2406

(301) 975-4239

(301) 975-4238

(303) 497-5766

(303) 497-3609

(303) 497-3609

(301) 975-2403

(301) 975-4230

(301) 975-2414

(301) 975-4230

(301) 975-2416

(303) 497-3743

(301) 975-5826

(303) 497-3634

(303) 497-3546

(301) 975-2420

(301) 975-2423

(303) 497-3538

(303) 497-3609

(301) 975-2426

(303) 497-3561

(303) 497-5362

(303) 497-3954

(303) 497-3634

(303) 497-3711

(301) 975-2423

(301) 975-5584

(301) 975-5559

(301) 975-6205

(301) 975-5538 
Mechanical Measurements

Acoustic

Acoustic Emission Transducer

Airspeed

Cryogenic Flow Rate

Flow Rate

Force

Mass

Ultrasonic Reference Block

Ultrasonic Transducer

Vibration

Optical Radiation Measurements

Laser Power/Energy

Photometric

Radiometric

Spectrophotometric

UV Radiometric-Standard Detectors

UV Radiometric-Standard Sources

Thermodynamic Measurements

Humidity

Laboratory Thermometers

Pressure

Radiation Thermometry

Resistance Thermometry

Thermocouples and Pyrometer Indicators

Vacuum and Low Pressure

Time and Frequency Measurements

Frequency Dissemination

Time Dissemination

Oscillator Characterization
Victor Nedzelnitsky

Franklin R. Breckenridge

Norman E. Mease

James A. Brennan

Kenneth R. Benson

Simone L. Yaniv

Jerry G. Keller

Gerald V. Blessing

Steven E. Fick

Myroslav R. Serbyn

Thomas R. Scott

Donald A. McSparron

John K. Jackson

P. Yvonne Barnes

L. Randall Canfield

J. Mervin Bridges

Gregory E. Scace

Jacquelyn A. Wise

B. Asoka Ratnam

Ronald L. Wilkinson

Gregory F. Strouse

George W. Burns

Richard W. Hyland

George Kamas

David W. Allan

James E. Gray
(301) 975-6638

(301) 975-6628

(301) 975-5959

(303) 497-3611

(301) 975-5945

(301) 975-6655

(301) 975-4218

(301) 975-6627

(301) 975-6629

(301) 975-6646

(303) 497-3651

(301) 975-2321

(301) 975-2330

(301) 975-2345

(301) 975-3728

(301) 975-3228

(301) 975-2626

(301) 975-4822

(301) 975-4857

(301) 975-2325

(301) 975-4803

(301) 975-4817

(301) 975-4829

(303) 497-3378

(303) 497-5637

(303) 497-3209

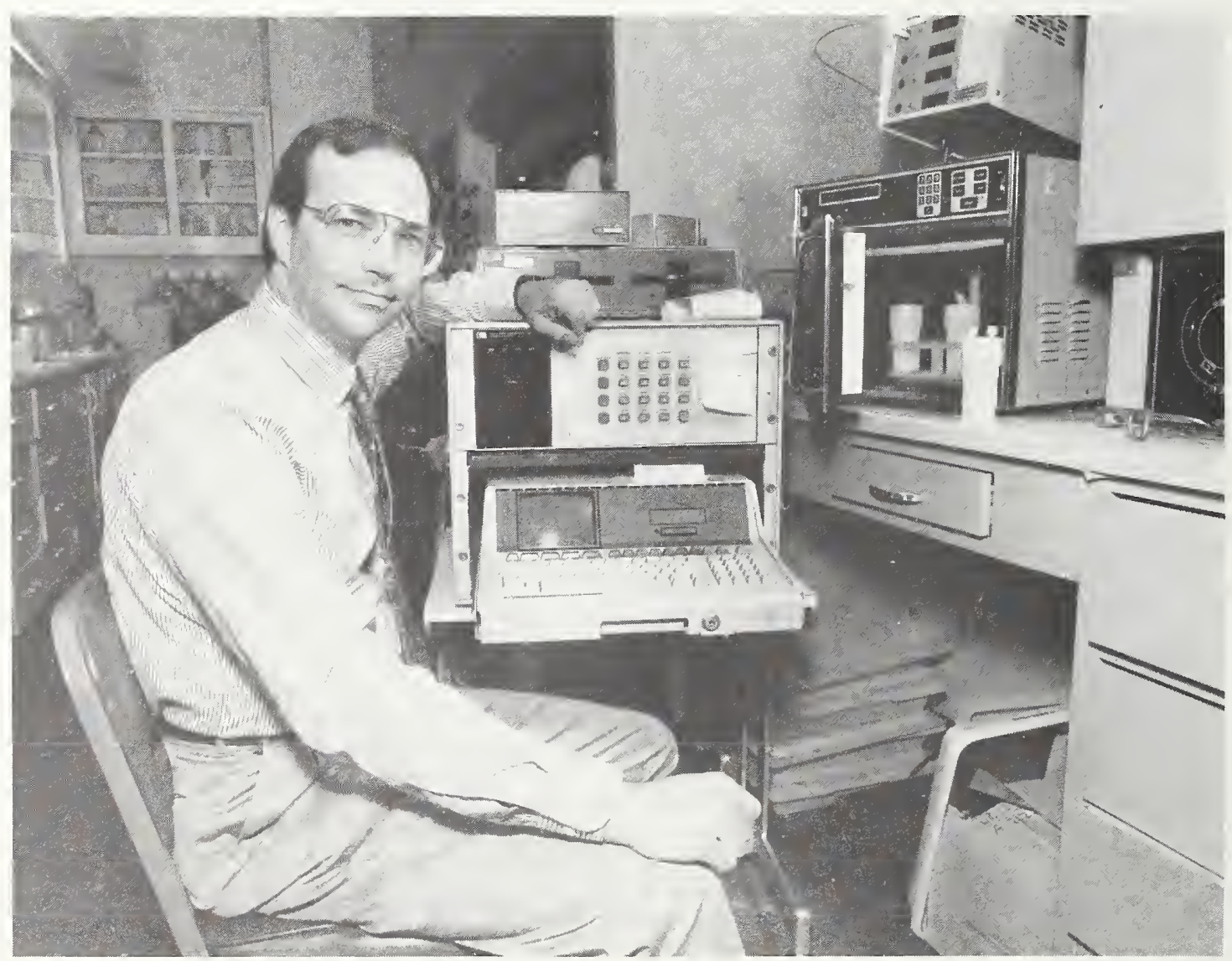

H. M. (Skip) Kingston of the Inorganic Analytical Research Division demonstrates the IR-100 award winning microwave dissolution system he led development of at NIST. 


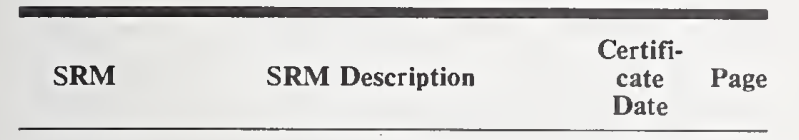

1c Limestone, Argillaceous - 78

4k Iron, Cast $\quad$ Mpr 79 27

5L Iron, Cast Nov $70 \quad 27$

$6 \mathrm{~g}$ Iron, Cast Nov $70 \quad 27$

$7 \mathrm{~g}$ Iron, Cast (High-Phosphorus) Oct 59

$8 \mathrm{j}$ Steel, Bessemer (Simulated) Apr $72 \quad 15$ $0.1 \mathrm{C}$

11h Steel, BOH, 0.2C

$12 \mathrm{~h}$ Steel, BOH, 0.4C

$13 \mathrm{~g}$ Steel, Carbon, $0.6 \mathrm{C}$

14 Steel, Carbon (AISI 1078)

15h Steel, BOH, 0.1C

$16 \mathrm{f}$ Steel, BOH, 1.0C

17d Sucrose (Polarimetric)

19h Steel, Basic Electric, 0.2C

$20 \mathrm{~g}$ Steel, AISI 1045

25d Ore, Manganese

27f Ore, Iron (Sibley)

30 Steel, Cr-V (SAE 6150)

$32 \mathrm{e}$ Steel, $\mathrm{Ni}-\mathrm{Cr}$

33 e Steel, Nickel

36b Steel, Cr2-Mo1

39i. Benzoic Acid, Calorimetric

$40 \mathrm{~h}$ Sodium Oxalate (Reductometric) 41c D-Glucose (Dextrose) (Polari-
metric)

43h Zinc Freezing Point

$44 \mathrm{f}$ Aluminum Freezing Point

45d Copper Freezing $\mathrm{Pt}$

$49 \mathrm{e} \quad$ Lead Freezing Point

50c Steel, W18-Cr4-V1 (Tool)

53 e Bearing Metal, Lead-base

57 a Silicon Metal

$58 \mathrm{a}$

$59 a$

$64 \mathrm{c}$

$68 \mathrm{c}$

$69 \mathrm{~b}$

$70 \mathrm{a}$

71

$72 \mathrm{~g}$

$73 \mathrm{c}$

$76 \mathrm{a}$

$77 \mathrm{a}$

$78 \mathrm{a}$

$79 \mathrm{a}$

$81 \mathrm{a}$

$82 b$

$83 d$

$84 \mathrm{j}$

$87 \mathrm{a}$

$88 \mathrm{~b}$

89

90

91
Ferrosilicon (73-Si, Regular Grade)

Ferrosilicon (48-Si)

Ferrochromium, High-Carbon

Ferromanganese, High-Carbon

Bauxite (Arkansas)

Feldspar (Potash)

Calcium Molybdate

Steel, Low-Alloy AISI 4130

Stainless Steel, $13 \mathrm{Cr}$

Burnt Refractory $\left(\mathrm{Al}_{2} \mathrm{O}_{3}-39\right)$

Burnt Refractory $\left(\mathrm{Al}_{2} \mathrm{O}_{3}-60\right)$

Burnt Refractory $\left(\mathrm{Al}_{2} \mathrm{O}_{3}-72\right)$

Fluorspar (Customs grade)

Sand, Glass (High Iron)

Iron, Cast, $\mathrm{Ni}-\mathrm{Cr}$

Arsenic Trioxide, Reductometric

Potassium Hydrogen Phthalate

Silicon-Aluminum Alloy

Limestone, Dolomitic

Glass, Lead-Barium

Ferrophosphorous

Glass, Opal (Powder)

Glass, Soda-Lime (Powder)

Glass, Borosilicate

Zn-base Die-Casting Alloy

Clay, Flint

Clay, Plastic
Apr $74 \quad 15$

Mar $66 \quad 15$

Apr $74 \quad 15$

Feb $81 \quad 15$

Mar $84 \quad 15$

Mar $83 \quad 15$

Aug $86 \quad 44$,

Sep $87 \quad 15$

Oct $70 \quad 15$

Feb $84 \quad 77$

May $77 \quad 76$

Jun $79 \quad 16$

Apr $57 \quad 16$

Nov $84 \quad 16$

Jul $69 \quad 16$

Jul $68 \quad 99$

May $82 \quad 44$

Nov $84 \quad 44$,

Aug $73 \quad 102$

Apr $73 \quad 102$

Dec $71 \quad 102$

Dec $71 \quad 102$

Jun $57 \quad 19$

Jan $70 \quad 34$

Dec $80 \quad 26$

Apr $78 \quad 26$

Nov $69 \quad 26$

Aug $77 \quad 26$

Aug $79 \quad 77$

Aug $81 \quad 79$

Jun $81 \quad 16$

Jul $66 \quad 19$

May $85 \quad 80$

May $85 \quad 80$

Jan $78 \quad 79$

Apr $66 \quad 27$

Mar 8244

Nov $84 \quad 44$

Aug $81 \quad 30$

Apr $86 \quad 79$

Aug $32 \quad 83$

Oct $28 \quad 26$

Oct $82 \quad 83$

Mar $82 \quad 83$

Aug $73 \quad 83$

Aug $73 \quad 38$

Apr $88 \quad 78$

Apr $88 \quad 78$
Aug $79 \quad 26$

Feb $29 \quad 26$

May $85 \quad 80$

Jan $80 \quad 75$

$\begin{array}{lcc}\text { SRM } & \text { Certifi- } \\ \text { cate } & \text { Page } \\ \text { Date }\end{array}$

$\begin{aligned} 99 \mathrm{a} & \text { Feldspar, Soda } \\ 100 \mathrm{~b} & \text { Steel, Mn-2 } \\ \text { 101g } & \text { Steel, Cr18-Nil0 (AISI 304L) } \\ 103 \mathrm{a} & \text { Chrome Refractory } \\ 105 & \text { Steel, High Sulfur }\end{aligned}$

$106 \mathrm{~b}$ Steel, $\mathrm{Cr}-\mathrm{Mo}-\mathrm{Al}$

107c Iron, Alloy Cast, Ni-Cr-Mo

$112 \mathrm{~b}$ Carbide, Silicon

$114 \mathrm{n}$ Portland Cement, Fineness

115 a Iron, Alloy Cast, $\mathrm{Cu}-\mathrm{Ni}-\mathrm{Cr}$

120c Phosphate Rock (Florida)

$121 \mathrm{~d}$

$122 \mathrm{~h}$

$123 \mathrm{c}$

$125 b$

$126 \mathrm{c}$

$127 \mathrm{~b}$

$129 \mathrm{c}$

$131 \mathrm{~d}$

$132 \mathrm{~b}$

$133 \mathrm{~b}$

$134 \mathrm{a}$

$136 \mathrm{e}$

$139 \mathrm{~b}$

$141 \mathrm{c}$

142

$143 \mathrm{c}$

148

$152 \mathrm{a}$

$153 \mathrm{a}$

$154 \mathrm{~b}$

155

$158 \mathrm{a}$

$160 \mathrm{c}$

163

$165 \mathrm{a}$

$166 \mathrm{c}$

$173 b$

176

178

180

181

182

183

$185 \mathrm{f}$

Stainless Steel, Cr17-Nil1Ti0.3 (AISI 321)

Iron, Cast, Car Wheel

Stainless Steel, Crl7-Ni11Nb0.6 (AISI 348)

Steel, High-Silicon (Ca-Bearing)

Steel, High-Nickel (Ni36)

Solder, Sn40-Pb60

Steel, High-Sulfur (SAE 112)

Aug $81 \quad 79$

Aug $59 \quad 16$

Aug $86 \quad 19$

Sep $62 \quad 81$

Aug $81 \quad 15$

Mar $61 \quad 16$

May $83 \quad 27$

Jan 8581

Feb $82 \quad 122$

Apr $62 \quad 27$

Feb $88 \quad 74$,

Aug $81 \quad 19$

Apr $83 \quad 27$

Jul $71 \quad 19$

Feb $82 \quad 16$

Dec $77 \quad 18$

Oct $81 \quad 34$

Aug $73 \quad 16$

Dec $86 \quad 16$

Steel, Tool (AISI M2) $\quad$ Aug 73 19

Steel, Chromium-Molybdenum Aug 81 19

Steel, Mo8-W2-Cr4-Vl May 5719

Potassium Dichromate Jun 8944

Steel, Cr-Ni-Mo (AISI 8640) May 78 16

Acetanilide

Anisic Acid

Cystine

Nicotinic Acid

Steel, BOH, 0.5C

Steel, Co8-Mo9-W2

Titanium Dioxide

Steel, Cr0.5-W0.5

Bronze, Silicon

Stainless Steel, Cr18-Ni12Mo2 (AISI 316)

Steel, Chromium

Sand, Glass

Stainless Steel, Low-C (AISI 316L)

Ti-Base Alloy 6Al-4V

Ti-Base Alloy 5A 1-2.5Sn

Steel, BOF 0.4C

Fluorspar, High-Grade

Ore, Lithium (Spodumene)

Ore, Lithium (Petalite)

Ore, Lithium (Lepidolite)

Sep $76 \quad 44$

Jul $69 \quad 44$

Sep $76 \quad 44$

Dec $70 \quad 44$

Oct $65 \quad 15$

Jan $60 \quad 19$

May $73 \quad 80$

Oct $46 \quad 16$

Aug 61 31

Aug $69 \quad 19$

Jan $68 \quad 16$

Oct $78 \quad 79$

Mar $70 \quad 19$

Dec $84 \quad 37$

Oct $81 \quad 37$

Jul $69 \quad 15$

Mar $71 \quad 75$

Oct $81 \quad 75$

Oct $81 \quad 75$

Oct $81 \quad 75$

Jan $84 \quad 89$

tassium Hydrogen Phthalate, $\mathrm{pH}$

186Id Potassium Dihydrogen Phosphate, $\mathrm{pH}$

186IId Disodium Hydrogen Phosphate, $\mathrm{pH}$

$187 \mathrm{c}$

Sodium Tetraborate Decahydrate (Borax), pH

Potassium Hydrogen Tartrate, $\mathrm{pH}$

$189 \mathrm{a}$

$191 \mathrm{a}$

$192 \mathrm{a}$

Potassium Tetroxalate, $\mathrm{pH}$

Sep 70

89

Sep $70 \quad 89$

Mar 84

89

Sodium Bicarbonate, $\mathrm{pH}$

Sodium Carbonate, pH

Potassium Nitrate

May $87 \quad 89$

Apr $86 \quad 89$ 


\section{Ammonium Dihydrogen Phos- phate \\ 195 Ferrosilicon (75Si)}

196

198

200

Ferrochromium (Low

Silica Brick $\left(0.16 \mathrm{~A} 1_{2} \mathrm{O}_{3}\right)$

Silica Brick $\left(0.48 \mathrm{Al}_{2} \mathrm{O}_{3}\right)$

Potassium Dihydrogen Phosphate

211c Toluene

276a Carbide, Tungsten

277 Tungsten Concentrate

278 Obsidian Rock

291 Steel, Cr-Mo (ASTM A-213)

Steel, Cr-Ni-Mo (AISI 8620)

Copper, Ore Mill Heads

Ore, Copper Mill Tails

Iron, Gray Cast

Steel, BOH, 0.1C

Steel, BOH, 1.1C

Iron, White Cast

Steel, Cr17-Ni9-Se0.2

Ferroniobium

Iron, Ductile Cast

Iron, Nodular Cast

Stainless Steel, Cr16-Ni2 (AISI 431)

Steel, Cr15-Ni7 (Mo precip harden)

Steel, Cr16-Ni4 (Cu precip harden)

Steel, Valve (Cr21-Ni3-Mn8)

High Temp Alloy A286 (Ni26Cr15)

Waspaloy

Benzoic Acid, Acidimetric

Titanium for Hydrogen

Zircaloy 2, Zr-Base Alloy

Steel, AISI 4340

Steel, AISI 94B17 (modified)

Steel, Cr-V (modified)

Steel, High C (modified)

Iron, Electrolytic

Stainless Steel (AISI 446)

Steel, AISI 1211

Zinc Oxide

Sulfur

Stearic Acid

Channel Black

Oil Furnace Black

Gas Furnace Black

Mercaptobenzothiazole

n-Tertiary-Butyl-2

Styrene Butadiene

Butyl Rubber

Copper " 0 "

Copper I

Copper II

Copper III

Copper V

Copper VI

Copper VII

Copper XI

Copper IV

Mineral Glasses

Optical Linewidth

AR Cr Optical Linewidth

B Cr Optical Linewidth

Glass Fluorescence Source

Tungsten-Molybdenum

Gold-Silver

Gold-Copper

SEM Magnification

5\% Austenite in Ferrite

$15 \%$ Austenite in Ferrite

$30 \%$ Austenite in Ferrite

2\% Austenite in Ferrite

Ferrite in Austenite (10\%)
Jan $74 \quad 74$

Jan $76 \quad 26$

Nov $70 \quad 26$

$\operatorname{Jan} 60 \quad 81$

Jan $60 \quad 81$

Aug $74 \quad 74$

Sep 8496

May $80 \quad 81$

Oct $78 \quad 75$

Aug $81 \quad 80$

Oct $75 \quad 16$

Mar $75 \quad 16$

Jan $77 \quad 75$

Jan $77 \quad 75$

Mar $82 \quad 27$

Apr $66 \quad 15$

Apr $85 \quad 15$

Jun $82 \quad 27$

Jul $65 \quad 19$

Nov $70 \quad 26$

Mar $62 \quad 27$

Apr $70 \quad 27$

Jul $85 \quad 19$

Oct $63 \quad 18$

Jan 64

Oct $85 \quad 18$

Mar $87 \quad 18$

Jun $87 \quad 35$

Apr $81 \quad 44$

Apr $86 \quad 38$

Feb $81 \quad 17$

Feb $81 \quad 17$

Feb $81 \quad 17$

Feb $81 \quad 17$

Feb $81 \quad 17$

Jul $77 \quad 19$

Jan $78 \quad 15$

none 121

none 121

none 121

none 121

none 121

none 121

none 121

none 121

Jan $85 \quad 121$

Mar $87 \quad 121$

Sep $80 \quad 33$

Jan $78 \quad 33$

Jan 78

Jan 78

Jan 78

Jan $78 \quad 33$

Jan $78 \quad 33$

Sep 80

Jan $78 \quad 33$

Oct 8141 ,

$\begin{array}{r}* \quad 42 \\ * \quad 91 \\ \hline\end{array}$

Apr 81 * 91

Feb $83 \quad 116$

Nov $68 \quad 41$

Feb 6941

Jun $69 \quad 41$

Oct $81 \quad 115$

Mar $81 \quad 115$

May $82 \quad 115$

Oct $83 \quad 115$
493

494

495

496

498

499

500

600

607

610

611

612

613

614

615

616

617

620

621

622

623

624

625

626

627

628

629

630

631

$640 \mathrm{~b}$

641

642

643

644

646

647

648

65

65

652

$654 a$

656

658

659

660

668

670

671

672

673

$674 \mathrm{a}$
675

675

676
677

679

$680 \mathrm{a}$

681

682

683

685

688

689

690

691

692

693

694

696

697

698

699

Iron Carbide in Ferrite

Copper I

Unalloyed Copper II

Unalloyed Copper III

Copper V

Unalloyed Copper VI

Copper VII

Bauxite (Australian) 


\begin{tabular}{|c|c|c|c|c|c|c|c|}
\hline SRM & SRM Description & $\begin{array}{c}\text { Certifi- } \\
\text { cate } \\
\text { Date }\end{array}$ & Page & SRM & SRM Description & $\begin{array}{c}\text { Certifi. } \\
\text { cate } \\
\text { Date }\end{array}$ & Page \\
\hline 705 & Polystyrene $179 \mathrm{k} \mathrm{mol} \mathrm{wt}$ & Nov 78 & $\begin{array}{l}97 \\
98\end{array}$ & 900 & $\begin{array}{l}\text { Antiepilepsy Drug Level } \\
\text { Assay }\end{array}$ & Apr 79 & 49 \\
\hline & & & 100 & 909 & Human Serum & Mar 85 & 49 \\
\hline 706 & Polystyrene $258 \mathrm{k} \mathrm{mol} \mathrm{wt}$ & Feb 79 & $\begin{array}{r}97 \\
98\end{array}$ & 910 & Sodium Pyruvate & May 81 & $\begin{array}{l}50 \\
49\end{array}$ \\
\hline 708 & $\begin{array}{l}\text { Glasses, Stress Optical Coeffi- } \\
\text { cient }\end{array}$ & Sep 73 & 95 & $\begin{array}{l}911 \mathrm{~b} \\
912 \mathrm{a}\end{array}$ & $\begin{array}{l}\text { Cholesterol } \\
\text { Urea }\end{array}$ & $\begin{array}{l}\text { May } 88 \\
\text { Nov } 79\end{array}$ & $\begin{array}{l}49 \\
49\end{array}$ \\
\hline 709 & Glass, Extra Dense Lead & Jun 74 & 95 & 913 & Uric Acid & Nov 73 & 49 \\
\hline $710 a$ & Glass, Soda Lime-Silica & & $\begin{array}{l}94, \\
95\end{array}$ & $\begin{array}{l}914 \mathrm{a} \\
915\end{array}$ & $\begin{array}{l}\text { Creatinine } \\
\text { Calcium Carbonate }\end{array}$ & $\begin{array}{r}\text { Oct } 87 \\
\text { Nov } 73\end{array}$ & $\begin{array}{l}49 \\
49\end{array}$ \\
\hline 711 & Glass, Lead-Silica & Jul 64 & 94, & $\begin{array}{l}916 \mathrm{a} \\
917 \mathrm{a}\end{array}$ & $\begin{array}{l}\text { Bilirubin } \\
\text { D-Glucose (Dextrose) }\end{array}$ & Sep 73 & $\begin{array}{l}49 \\
49\end{array}$ \\
\hline 712 & Glass, Alkali Lead-Silica & Oct 66 & 95 & 918 & Potassium Chloride & Nov 73 & 49 \\
\hline 713 & Glass, Dense Barium Crown & Oct 66 & 95 & $919 \mathrm{a}$ & Sodium Chloride & Nov 73 & 49 \\
\hline 714 & Glass, Alkali Alumina Silica & Oct 66 & 95 & 920 & D-Mannitol & Nov 73 & 49 \\
\hline 715 & Glass, Alkali-free Alumina & Sep 66 & 95 & 921 & Cortisol (Hydrocortisone) & Dec 73 & 49 \\
\hline 716 & Glass, Neutral & Sep 66 & 95 & 922 & Tris(hydroxymethyl) amino- & Aug 76 & 89 \\
\hline 717 & Glass, Borosilicate & Nov 69 & $94-$ & & methane, $\mathrm{pH}$ & & \\
\hline & & & 95 & 923 & Tris(hydroxymethyl) amino- & Aug 76 & 49 , \\
\hline $723 a$ & $\begin{array}{l}\text { Tris(hydroxymethyl) amino- } \\
\text { methane, Basimetric }\end{array}$ & Apr 81 & 44 & 924 & $\begin{array}{l}\text { methane hydrochloride, } \mathrm{pH} \\
\text { Lithium Carbonate }\end{array}$ & Nov 73 & $\begin{array}{l}89 \\
49\end{array}$ \\
\hline $724 a$ & $\begin{array}{l}\text { Tris(hydroxymethyl) amino- } \\
\text { methane, Calorimetric }\end{array}$ & Sep 73 & 99 & 925 & $\begin{array}{l}\text { 4-Hydroxy-3-methoxy-dl- } \\
\text { mandelic Acid (VMA) }\end{array}$ & Dec 73 & 49 \\
\hline 726 & Selenium, Inter-Purity & Jan 67 & 40 & 926 & Bovine Serum Albumin (Total & Jul 77 & 49 \\
\hline 728 & Zinc-Intermediate Purity & Oct 81 & 40 & & Protein) & & \\
\hline 731 & Glass, Borosilicate & Jul 72 & 104 & $927 \mathrm{a}$ & Bovine Serum Albumin $17 \%$ & Aug 86 & 49 \\
\hline 738 & Stainless Steel & Nov 86 & 104 & & Solution, Total Protein) & & \\
\hline 739 & Fused Silica & May 71 & 104 & 928 & Lead Nitrate & May 76 & 49 \\
\hline $740 \mathrm{a}$ & Zinc Freezing Point & Feb 70 & 102 & 929 & Magnesium Gluconate Dihy- & Apr 79 & 49 \\
\hline 741 & Tin Freezing Point & Jul 72 & 102 & & drate & & \\
\hline 742 & Alumina Melting Point & Jul 70 & 102 & $930 \mathrm{D}$ & Glass Filters for Spectrophoto- & Aug 84 & 106 \\
\hline 743 & Mercury, Triple Point & Apr 76 & 102 & & metry (Visible) & & \\
\hline 745 & Gold, Vapor Pressure & May 69 & 102 & $931 d$ & Liquid Absorbance Filters for & Oct 86 & 106 \\
\hline 746 & Cadmium, Vapor Pressure & Aug 70 & 102 & & UV and Visible Spectropho- & & \\
\hline 748 & Silver, Vapor Pressure & Aug 70 & 102 & & tometry & & \\
\hline 763 & $\begin{array}{l}\text { Aluminun, Magnetic Suscepti- } \\
\text { bility }\end{array}$ & Apr 73 & 105 & 934 & $\begin{array}{l}\text { Clinical Laboratory Thermo- } \\
\text { meter }\end{array}$ & * & 103 \\
\hline 766 & Manganese Fluoride, Mag Sus- & Apr 73 & 105 & $935 \mathrm{a}$ & $\begin{array}{l}\text { Crystalline Potassium Dichro- } \\
\text { mate for UV Absorbance }\end{array}$ & Jun 77 & 106 \\
\hline $767 a$ & Thermometric Fix Point & Jun 83 & 101 & $\begin{array}{l}936 \\
937\end{array}$ & $\begin{array}{l}\text { Quinine Sulfate Dihydrate } \\
\text { Iron Metal }\end{array}$ & $\begin{array}{l}\text { Apr } 79 \\
\text { Jun } 78\end{array}$ & $\begin{array}{r}106 \\
49\end{array}$ \\
\hline 769 & $\begin{array}{c}\text { Device } \\
\text { Electrical "RRR" Set }\end{array}$ & & & 938 & 4-Nitrophenol & May 81 & 49 \\
\hline 772 & $\begin{array}{l}\text { Electrical "RRR" Set } \\
\text { Nickel, Magnetic Moment }\end{array}$ & $\begin{array}{r}\text { Nov } 82 \\
\text { Oct } 78\end{array}$ & $\begin{array}{l}119 \\
105\end{array}$ & 951 & Boric Acid & Oct 71 & 44 \\
\hline 773 & Glass, Liquidus Temperature & Nov 80 & 95 & 952 & & & $\begin{array}{l}88 \\
88\end{array}$ \\
\hline 774 & Glass, Dielectric Constant & Ju1 82 & 94 & $\begin{array}{l}952 \\
953\end{array}$ & $\begin{array}{l}\text { Enriched Boric Acid } \\
\text { Neutron Density Monitor Wire }\end{array}$ & $\begin{array}{l}\text { Mar } 69 \\
\text { Mat }\end{array}$ & $\begin{array}{l}88 \\
87\end{array}$ \\
\hline 853 & Aluminum Alloy 3004 & May 85 & 30 & 956 & Electrolytes in Serum for ISE & * & 49 \\
\hline 854 & Aluminum Alloy 5182 & May 85 & 30 & $963 \mathrm{a}$ & Fission Track Glass (U-1 ppm) & Feb 84 & 87 \\
\hline 855 & Aluminum Casting Alloy 356 & Jan 80 & 30 & 968 & Fat Soluble Vitamins in & Apr 88 & 49 \\
\hline 856 & Aluminum Casting Alloy 380 & Jan 80 & 30 & 900 & Human Serum & & \\
\hline 858 & Aluminum Alloy 6011 (mod) & Jun 80 & 30 & 975 & Chlorine, Isotopic & Mar 65 & 88 \\
\hline 859 & Aluminum Alloy 7075 & Jun 80 & 30 & 976 & Copper, Isotopic & Mar 65 & 88 \\
\hline 864 & Inconel 600 & May 84 & 35 & 977 & Bromine, Isotopic & Mar 65 & 88 \\
\hline 865 & Inconel 625 & May 84 & 35 & $978 \mathrm{a}$ & Silver, Isotopic & Sep 84 & 88 \\
\hline 866 & Incoloy 800 & May 84 & 35 & 979 & Chromium, Isotopic & May 66 & 88 \\
\hline 867 & Incoloy 825 & May 84 & 35 & 980 & Magnesium, Isotopic & Jan 67 & 88 \\
\hline 868 & High-Temperature Alloy (Fe- & May 87 & 18 & 981 & Lead, Common Isotopic & Apr 73 & 88 \\
\hline & $\mathrm{Ni}-\mathrm{Co})$ & & & 982 & Lead, Equal-Atom Isotopic & Jun 68 & 88 \\
\hline 871 & Phosphor Bronze, CDA 521 & Aug 79 & 31 & 983 & Lead, Radiogenic Isotopic & Jun 68 & 88 \\
\hline 872 & Phosphor Bronze, CDA 544 & Aug 79 & 31 & 984 & Rubidium Chloride, Assay \& & Jul 70 & 88 \\
\hline 874 & Cupro-Nickel, 10 (CDA 706) & Jan 78 & 31 & & Isotopic & & \\
\hline & (pure) & & & 985 & Potassium, Assay \& Isotopic & Aug 79 & 88 \\
\hline 875 & Cupro-Nickel, 10 (CDA 706) & Jan 78 & 31 & 986 & Nickel, Isotopic & & 88 \\
\hline & (doped) & & & 987 & Strontium, Assay \& Isotopic & Oct 82 & 44 , \\
\hline 879 & Nickel Silver, CDA 762 & Jun 79 & 31 & & & & 88 \\
\hline 880 & Nickel Silver, CDA 770 & Jun 79 & 31 & 989 & Rhenium, Assay \& Isotopic & Feb 74 & 88 \\
\hline 882 & $\mathrm{Ni}-\mathrm{Cu}$ Alloy $(65 \mathrm{Ni}-31 \mathrm{Cu}-3 \mathrm{Al})$ & Aug 79 & 35 & 990 & Silicon, Assay \& Isotopic & Aug 75 & 88 \\
\hline 887 & Cemented Carbide & Sep 88 & 82 & 991 & Lead-206 Spike, Assay \& Iso- & Mar 76 & 88 \\
\hline 888 & Cemented Carbide & Sep 88 & 82 & & topic & & \\
\hline 889 & Cemented Carbide & Sep 88 & 82 & 994 & Gallium, Isotopic & Dec 85 & 88 \\
\hline 890 & Iron, HA White Cast (HC- & Apr 82 & 27 & 997 & Thallium, Isotopic & Jan 86 & 88 \\
\hline & $250+V)$ & & & 998 & Angiotensin I (Human) & Jan 83 & 49 \\
\hline 891 & Iron, HA White Cast (Ni-Hard & Apr 82 & 27 & 999 & Potassium Chloride (Primary) & Sep 72 & 44 \\
\hline & & & & 1001 & X-Ray Film Step Tablet $(0-4)$ & Jun 86 & 125 \\
\hline 892 & Iron, HA White Cast (Ni-Hard & Apr 82 & 27 & $1002 \mathrm{c}$ & Surface Flammability & Dec 78 & 128 \\
\hline & IV) & & & $1003 a$ & Glass Spheres $(8-58 \mu \mathrm{m})$ & Sep 84 & 122 \\
\hline 897 & Tracealloy A & Aug 83 & 36 & $1004 a$ & Glass Beads (34-120 $\mu \mathrm{m})$ & * & 122 \\
\hline 898 & Tracealloy B & Aug 83 & 36 & $1006 c$ & Smoke Density, Nonflame (cel- & Apr 83 & 128 \\
\hline 899 & Tracealloy C & Aug 83 & 36 & & lulose) & & \\
\hline & & & & $1007 \mathrm{a}$ & $\begin{array}{l}\text { Smoke Density, Flame (ABS } \\
\text { plastic) }\end{array}$ & Feb 76 & 128 \\
\hline & & & & 1008 & Photographic Step Tablet $(0-4)$ & Jun 86 & 125 \\
\hline
\end{tabular}




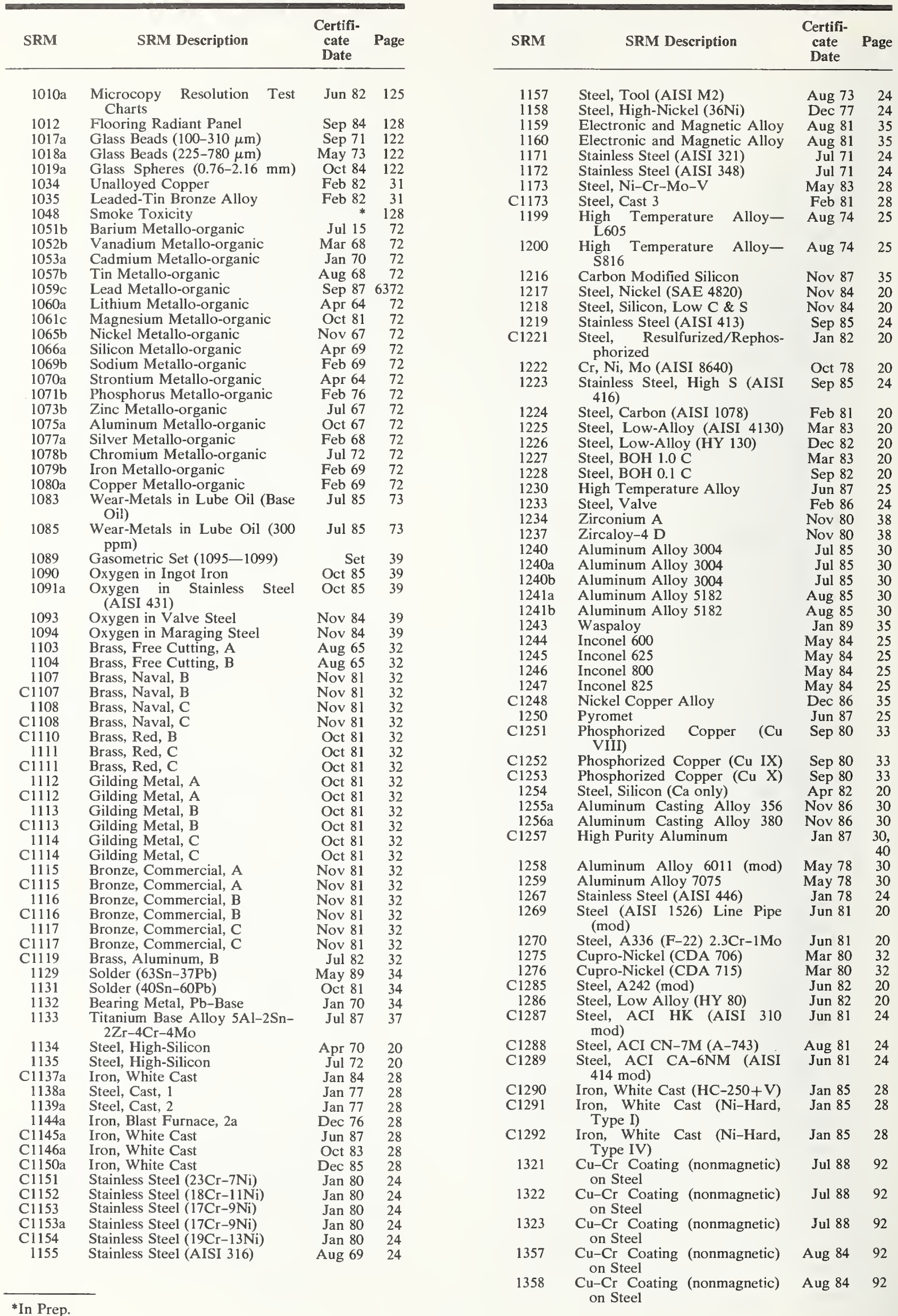


$1359 \mathrm{Cu}-\mathrm{Cr}$ Coating (nonmagnetic) May $84 \quad 92$ on Steel

$1360 \mathrm{Cu}-\mathrm{Cr}$ Coating (nonmagnetic) May 8492 on Steel

1361a $\mathrm{Cu}-\mathrm{Cr}$ Coating (nonmagnetic) May 849 on Steel

1362a $\mathrm{Cu}-\mathrm{Cr}$ Coating (nonmagnetic) May 8492 on Steel

1363a Cu-Cr Coating (nonmagnetic) May 849 on Steel

1364a $\mathrm{Cu}-\mathrm{Cr}$ Coating (nonmagnetic) on Steel

1365a Nickel (magnetic) on Steel May 84

1366a Nickel (magnetic) on Steel May 84

1379 Ultra-thin Gold on Nickel 0.35 May 8493 mg

1380 Ultra-thin Gold on Nickel 0.55 mg

1387 Gold Coating on Nickel 2.2

May $84 \quad 93$

1399b Gold Coating on Nickel (set)

1411 Soft Borosilicate Glass

1412 Multicomponent Glass

1413 Glass Sand, High Alumina

1449 Fumed Silica Board

1450b Thermal Resistance, Fibrous Glass Board

1451 Thermal Resistance, Fibrous Glass Blanket

1452 Thermal Resistance, Fibrous Glass Batt

1457 Superconducting Critical Current $\mathrm{Nb}-\mathrm{Ti}$ Wire

1459 Fumed Silica Board

1461 Thermal Conductivity and Electrical Resistivity, Stainless Steel

1462 Thermal Conductivity and Electrical Resistivity, Stainless Steel

1470 Gas Transmission, Polyester Film

1474 Polyethylene Melt Flow

1475 Linear Polyethylene $(52 \mathrm{k} \mathrm{mol}$ wt)

1476 Branched Polyethylene (viscosity)

1478 Polystyrene (37k mol wt)

1479 Polystyrene (1M mol wt)

1480 Polyurethanes Low MW

1481 Polyurethanes High MW

1482 Linear Polyethylene (13k mol wt)

1483 Linear Polyethylene $(32 \mathrm{k} \mathrm{mol}$ wt)

1484 Linear Polyethylene (119k mol wt)

1487 Poly (methylmethacrylate),

1488 Poly (methylmethacrylate)

1489 Poly (methylmethacrylate)

1490 Polyisobutylene Solution in Cetane, Rheology

1491 Aromatic Hydrocarbons in Hexane

Chlorinated Pesticides in Hexane

Polychlorinated Bipheny

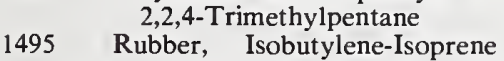
(Butyl) (Low Mooney Viscosity)

1496
Sep 8593

May $84 \quad 93$

Aug $85 \quad 83$

Aug $85 \quad 83$

Aug $85 \quad 79$

Jan $89 \quad 104$

May $85 \quad 104$

May $85 \quad 104$

Apr 86104

Jun $84 \quad 119$

Jan 89104

May $84 \quad 104$,

118

May $84 \quad 104$,

118

Feb $82 \quad 117$

* 97

Dec $78 \quad 97$,

98,

Nov $69 \quad 97$

Jam $79 \quad 98$

98

Mar $81 \quad 97$,

98
$* \quad 97$

$\begin{array}{rr}* & 97 \\ \text { Oct } 76 \quad 97,\end{array}$

98
-96

Mar $76 \quad 97$,

Oct $76 \quad 97$

98

97,
98

Feb 8897

Mar $86 \quad 97$

98

Aug 8963

Apr $89 \quad 64$

65

undated 63

Mar $81 \quad 121$

Sep $88 \quad 97$
1497

1507

1508

1514

1515

1543
1547

1548

1549

1563

$1566 \mathrm{a}$

$1567 \mathrm{a}$

$1568 \mathrm{a}$

1569

1572

$1573 \mathrm{a}$

1575

$1577 \mathrm{a}$

1579

1580

1581

1582

1583

1584

1585

1586

1587

1588

1589

1590

1595

1596

1597

1598

1599

1600

1614

1616

1617

1618

1619

$1620 \mathrm{~b}$

$1621 \mathrm{c}$

$1622 \mathrm{c}$

$1623 \mathrm{~b}$

$1624 \mathrm{~b}$

1625

1626

1627

1630
Polythylene Resin (Pigmented))

THC in Freeze-Dried Urine

Cocaine in Urine

Jul $87 \quad 97$

Thermal Analysis Purity (DSC)

Apple Leaves

GC/MS System Performance

Peach Leaves

Total Diet

Non-Fat Milk Powder

Cholesterol and Fat-Soluble

Vitamins in Coconut Oil

Oyster Tissue

Wheat Flour

Rice Flour

Brewers Yeast (Cr only)

Citrus Leaves

Tomato Leaves

Pine Needles

Bovine Liver

Powdered Lead-Based Paint ( $\mathrm{Pb}$ only)

Organics in Shale Oil

Polychlorinated Biphenyls in Oil

Petroleum Crude Oil

Chlorinated Pesticides in 2,2,4Trimethylpentane

Priority Pollutant Phenols in Methanol

Chlorinated Biphenyls

Isotopically Labeled and Unlabeled Priority Pollutants in Methanol

Nitrated Polycyclic Aromatic Hydrocarbons in Methanol

Organics in Cod Liver Oil

PCB's in Human Serum

Stabilized Wine

Tripalmitin

Dinitropyrene Isomers and INitropyrene in Methylene Chloride

Complex Mixture of Polycyclic Aromatic Hydrocarbons

Inorganic Constituents in Bovine Serum

Anticonvulsant Drug Level Assay

Secondary Standard Magnetic Tape Cassette (Computer Amplitude)

Dioxin in Isooctane

Sulfur in Kerosene

Sulfur in Kerosene

$\mathrm{V}$ and $\mathrm{Ni}$ in Residual Fuel Oil

Sulfur in Residual Fuel Oil $(0.7 \%)$

Sulfur in Residual Fuel Oil $(4.5 \%)$

Sulfur in Residual Fuel Oil $(0.9 \%)$

Sulfur in Residual Fuel Oil $(1.9 \%)$

Sulfur in Residual Fuel Oil $(0.2 \%)$

Sulfur in Distillate (Diesel) Fuel Oil $(0.1 \%)$

Sulfur Dioxide Permeation Tube, $10 \mathrm{~cm}$

Sulfur Dioxide Permeation Tube, $5 \mathrm{~cm}$

Sulfur Dioxide Permeation Tube, $2 \mathrm{~cm}$

Trace Mercury in Coal

$\begin{array}{r}* \quad 68 \\ \hline \quad 68\end{array}$

$* 53$

Aug $84 \quad 67$

undated 51

Jul $85 \quad 5$

Jul $87 \quad 52$

Oct $89 \quad 51$

Sep $88 \quad 51$

Jan 7851

Sep $76 \quad 51$

Dec 8253

Oct $76 \quad 53$

Feb $85 \quad 51$

Jan $73 \quad 59$

Nov $80 \quad 63$,

Jan $82 \quad 64$

Feb $85 \quad 63$

Apr $84 \quad 63$

Oct 8463

Jun 8563 ,

Jan 89

63,
67

Nov $85 \quad 49$,

67
Dec $80 \quad 52$

Jul 8349

Jul 8763 ,

Sep $87 \quad 63$,

64

Aug $82 \quad 49$

Mar $74 \quad 126$

Jul $85 \quad 63$

Feb $88 \quad 61$

Feb $88 \quad 61$

May $85 \quad 59$

Dec 81

61 
1632b Trace Elements in Coal (Bituminous) 1633a Trace Elements in Coal Fly

1634b Trace Elements in Fuel Oil

1635 Trace Elements in Coal (Subbituminous)

1639 Halocarbons (in methanol) for Water Analysis

$1641 \mathrm{~b}$ Mercury in Water $(\mu \mathrm{g} / \mathrm{mL})$

1643

Trace Elements in Water

Estuarine Sediment

1647a Priority Pollutant Polynuclear Aromatic Hydrocarbons

1648 Urban Particulate Matter

1649 Urban Dust/Organics

1650 Diesel Particulate Matter

1651

Heat-Source Powder for Calorimetry Zirconium-Barium Chromate, 1460

1652 Heat-Source Powder for Calorimetry Zirconium-Barium Chromate, 1632

1653 Heat Source for Calorimetry Zirconium-Barium Chromate, 1762

1655 Potassium Chloride for Solution Calorimetry

1656 Thianthrene, Combustion $\mathrm{Ca}$ lorimetry

1657 Synthetic Refuse-Derived Fuel, Combustion Calorimetry

$1658 \mathrm{a}$

$1659 \mathrm{a}$

$1660 \mathrm{a}$

Methane in Air, lppm

Methane in Air, $10 \mathrm{ppm}$

Methane (4) and Propane (1) in Air

1661a Sulfur Dioxide in Nitrogen, 500 ppm

1662a Sulfur Dioxide in Nitrogen, $1000 \mathrm{ppm}$

1663a Sulfur Dioxide in Nitrogen, $1500 \mathrm{ppm}$

1664a Sulfur Dioxide in Nitrogen, $2500 \mathrm{ppm}$

1665b Propane in Air, 3 ppm

$1666 \mathrm{~b}$ Propane in Air, $10 \mathrm{ppm}$

1667b Propane in Air, $50 \mathrm{ppm}$

$1668 \mathrm{~b}$ Propane in Air, $100 \mathrm{ppm}$

$1669 \mathrm{~b}$ Propane in Air, $500 \mathrm{ppm}$

1670

Carbon Dioxide in 330. ppm

Jun 85

62

Jan $85 \quad 62$

Feb $86 \quad 62$

Aug $79 \quad 62$

Aug $86 \quad 59$

Apr 83 63,

Apr $83 \quad 59$

May $84 \quad 62$

Jun $82 \quad 62$

Dec 81 63,

May $82 \quad 62$

Apr 8263

Feb $85 \quad 63$,

Nov $68 \quad 64$

Nov $68 \quad 99$

Nov $68 \quad 99$

Mar $81 \quad 99$

Jan $85 \quad 99$

Mar $85 \quad 99$

Mar $81 \quad 55$

Mar $81 \quad 55$

Mar $81 \quad 55$

Oct $88 \quad 56$

Oct $88 \quad 56$

Mar $81 \quad 56$

Dec $87 \quad 56$

Dec 8756

Dec 8756

Jan $80 \quad 56$

Jan $80 \quad 56$

Dec 8254

1671 Carbon Dioxide in Air, Dec 82 54 340 ppm

1672 Carbon Dioxide in Air, Dec 8254 350 ppm Carbon Dioxide in Nitrogen,
$7 \%$

1675b Carbon Dioxide in Nitrogen, $14 \%$

1677c Carbon Monoxide in Nitrogen, $10 \mathrm{ppm}$

1678c Carbon Monoxide in Nitrogen, $50 \mathrm{ppm}$

1679c Carbon Monoxide in Nitrogen, $100 \mathrm{ppm}$

1680b Carbon Monoxide in Nitrogen, $500 \mathrm{ppm}$

1681b Carbon Monoxide in Nitrogen, $1000 \mathrm{ppm}$

1683b Nitric Oxide in Nitrogen, $50 \mathrm{ppm}$

$1684 b$

Nitric Oxide in Nitrogen, $100 \mathrm{ppm}$

$1685 b$

Nitric Oxide $250 \mathrm{ppm}$

$1686 \mathrm{~b}$

Nitric Oxide $500 \mathrm{ppm}$

$1687 b$
Jan $80 \quad 54$

$\operatorname{Jan} 80 \quad 54$

Jan $80 \quad 55$

Jan $80 \quad 55$

Jan $80 \quad 55$

Jan $80 \quad 55$

Jan $80 \quad 55$

Nov $88 \quad 55$

Nov $88 \quad 55$

Jan $80 \quad 55$

Jan $80 \quad 55$

Jan $80 \quad 55$
Mar $81 \quad 56$

169

Polystyrene Spheres, $0.3 \mu \mathrm{m}$

Sulfur Dioxide in Nitrogen, $50 \mathrm{ppm}$

1694a Sulfur Dioxide in Nitrogen, $100 \mathrm{ppm}$

1696 Sulfur Dioxide in Nitrogen, $3500 \mathrm{ppm}$

1700a Carbon Dioxide in Nitrogen, 10\% Blood Gas

1701a Carbon Dioxide and Oxygen in Nitrogen, $5 \%$ and $12 \%$, Blood Gas

1702a Carbon Dioxide and Oxygen in Nitrogen, $5 \%$ and $20 \%$, Blood Gas

1703a Carbon Dioxide and Oxygen in Nitrogen, $10 \%$ and $7 \%$, Blood Gas Nitric Oxid
$1000 \mathrm{ppm}$
1754

1761

1762

1763

1764

1765

1766

1767

1804

1805

1806

1808

1809

1810 a

1811

1812

1813

1814

$1815 a$

$1816 \mathrm{a}$

$1817 \mathrm{~b}$

1818

1819

1822

1823

1825

1826

1827

1828

1829

1830

1831

1832

1833

Steel, Low Alloy (AISI 4320)

Steel, Low Alloy

Steel, Low Alloy

Steel, Low Alloy

Steel, Low Alloy

Steel, Low Alloy

Steel, Low Alloy

Steel, Low Alloy

Ambient Toxic Organics in Nitrogen

Benzene in Nitrogen, $0.25 \mathrm{ppm}$

Benzene in Nitrogen, 10 ppm

Tetrachloroethylene in Nitrogen, $0.25 \mathrm{ppm}$

Tetrachloroethylene in Nitrogen, $10 \mathrm{ppm}$

Linerboard

Aromatic 'Gases in Nitrogen $0.25 \mathrm{ppm}$

Aromatic Gases in Nitrogen 10 ppm

Aliphatic Organic Gases in Nitrog ${ }^{` n} 0.25 \mathrm{ppm}$

Aliphatic Organic Gases in Nitrogen $10 \mathrm{ppm}$

n-Heptane, Reference Fuel

Isooctane, Reference Fuel

Catalyst Package for Lubricant Oxidation

Chlorine in Lube Base Oil

Sulfur in Lubricating Base Oil

Refractive Index Glass, SodaLime

Refractive Index Silicone Liquids

Glass Density

Glass Density

Glass Density

Ethanol-Water Solutions

Alcohols in Reference Fuel

Soda-Lime Float Glass

Soda-Lime Sheet Glass

Thin Glass Film on Polycarbonate for X-ray Fluorescence

Thin Glass Film on Polycarbonate for X-ray Fluorescence

1834

1835

1836

1837

1838

1839

$1841 \mathrm{a}$

1845

Borate Ore

Nitrogen in Lube Base Oil

Methanol and t-Butanol in Reference Fuels

Ethanol in Reference Fuels

Methanol in Reference Fuels

Silicon Density, $200 \mathrm{~g}$

Cholesterol In Whole Egg Powder

1850
Fused Ore Glass for XRF

Dec $82 \quad 122$

May $84 \quad 122$

Jun $89 \quad 56$

Jun $89 \quad 56$

Jul $84 \quad 56$

Aug $88 \quad 49$,

Aug $88 \quad 49$

Aug $88 \quad 49$

Aug $88 \quad 49$

Feb $89 \quad 39$

Feb $88 \quad 20$

Feb $88 \quad 20$

Feb $88 \quad 20$

Feb $88 \quad 20$

Feb $88 \quad 20$

Feb $88 \quad 20$

Feb $88 \quad 20$

Dec $82 \quad 54$

Dec $82 \quad 54$

Jun $83 \quad 57$

Jun $83 \quad 57$

Dec $83 \quad 128$

Nov $85 \quad 54$

Nov $85 \quad 54$

Mar $87 \quad 54$

Mar $87 \quad 54$ 


SRM SRM Description $\begin{gathered}\text { Certifi- } \\ \text { cate } \\ \text { Date }\end{gathered}$ Page

1851

1855

1856

1857

1860

1862

1866

1867

1871 sis
$\mathrm{Pb}-\mathrm{Ge}$ Glasses for Microanalysis

$1873 \mathrm{Ba}-\mathrm{Zn}$-Si Glasses for Microanalysis

1874 Li-Al-Bo Glasses for Microanalysis

1875 Al-Mg-P Glasses for Microanalysis

$\begin{array}{ll}\text { 1876a } & \text { Chrysotile Asbestos Fibers } \\ 1878 & \text { Respirable Alpha Quartz }\end{array}$

1880 Portland Cement, black

1881 Portland Cement, white

$1882 \mathrm{Ca}-\mathrm{Al}$ Cement

1883 Ca-Al Cement

1884 Cement Composition

1885 Cement Composition

1886 Cement Composition

1887 Cement Composition

1888 Cement Composition

1889 Cement Composition

1890 Stainless Steel for Pitting of Crevice Corrosion

1891 Co-Cr-Mo Alloy for Pitting of Crevice Corrosion

$1893 \mathrm{Cu}$ Microhardness Knoop

1894 Cu Microhardness Vickers

1895 Ni Microhardness Knoop

1896 Ni Microhardness Vickers

1901 Centerline Drawings for Optical Character RecognitionType B

1905 Ni Microhardness Knoop-300

1906 Ni Microhardness Knoop-500

1907 Ni Microhardness Knoop-1000

1920 Near Infrared Reflectance Wavelength

1923 Polystyrene Sulfonates

1924 Polystyrene Sulfonates

1925 Polystyrene Sulfonates

1930 Glass Filters, Transmittance

1931 Fluorescence Corrected Emission Spectrum

1939 Polychlorinated Biphenyls in Sediment

1940 Polychlorinated Biphenyls in Sediment

1941 Organics in Marine Sediment

1951a Cholesterol in Human Serum (Frozen)

1952a Cholesterol in Human Serum (Freeze-dried)

1960 Polystyrene Spheres, $10 \mu \mathrm{m}$

1961 Polystyrene Spheres, $30 \mu \mathrm{m}$

1962 Polystyrene Spheres, $0.3 \mu \mathrm{m}$

1965 Polystyrene Spheres, $10 \mu \mathrm{m}$ moelement

1968 Gallium Melting Point

1969 Rubidium Triple Point

1970 Succinonitrile Triple Point

1971

1974

1975

2003

Indium Melting Point

Organics in Mussel Tissue

Diesel Particulate Bioassay face, Reflectance

2009a Didymium Glass Filter, Wavelength
1879 Respirable Cristobalite

1967 High-Purity Platinum Ther-

Aluminum Mirror, First Sur-
Apr $84 \quad 123$

Jan $86 \quad 97$

Jul $85 \quad 97$

Mar $83 \quad 115$

Aug $82 \quad 119$

Aug $82 \quad 119$

Nov 8871

* 71

May $84 \quad 41$,

May $84 \quad 41$

May $84 \quad 42$

42

Dec 84 41,

Dec 8441 ,

Jun $83 \quad 72$

Nov $83 \quad 70$

Jan $88 \quad 70$

Feb $84 \quad 84$

Feb $84 \quad 84$

Jul $86 \quad 84$

Jul $86 \quad 84$

Sep $89 \quad 84$

Sep $89 \quad 84$

Sep $89 \quad 84$

Sep $89 \quad 85$

Sep $89 \quad 85$

Sep $89 \quad 85$

May $83 \quad 116$

Sep $85 \quad 116$

Feb $84 \quad 96$

Feb $84 \quad 96$

Feb $84 \quad 96$

Feb $84 \quad 96$

Mar $76 \quad 126$

Aug $86 \quad 96$

Sep $86 \quad 96$

Sep $86 \quad 96$

Jul $86 \quad 107$

* 97

$*$
$* \quad 97$
$* \quad 97$

Mar $87 \quad 106$

* 106

* 63

* 63

* 63

* 49

* 49

Apr $85 \quad 122$

Jan $87 \quad 122$

Jan $87 \quad 122$

Feb $77 \quad 103$

Jun $77 \quad 102$

Jan $84 \quad 102$

Mar $85 \quad 102$

Feb $87 \quad 102$

$* 63$
$* \quad 63$

May $85 \quad 107$

Jul 84106
2011

2015

Gold Mirror, First Surface, Reflectance

2016

Spectral Reflectance

White Opal Glass

2021

Black Porcelain Enamel, Directional-Hemispherical $\mathrm{Re}$ flect

2023

Aluminum Mirror, Second Surface, Reflectance

2025 Aluminum Mirror with Wedge, Second Surface, Reflectance

2031 Metal-on-Quartz Filters for Spectrophotometry

2032 Potassium Iodide Stray Light

2033 KI Stray Light with Attenuator

2034 Holmium Oxide Solution Wavelength

2063 Microanalysis Thin Film Mg$\mathrm{Si}-\mathrm{Ca}-\mathrm{Fe}$

2064

Thin Film Microanaylsis

2069a

2071

2072

2073

2074

2075

2083

2092

2096

2106

$2135 \mathrm{c}$

2136

2137

2141

2142

2143

2144

2151

2152

2161

2162

2163

2164

2165

2166

2167

2168

2181

2182

2185

$2186 \mathbf{I}$

SEM Performance Standard

Sinusoidal Roughness

Sinusoidal Roughness

Sinusoidal Roughness

Sinusoidal Roughness

Sinusoidal Roughness

Socketed Ball Bar

Charpy V-Notch Low Energy

Charpy V-Notch High Energy

Centroid Color Charts

$\mathrm{Ni} / \mathrm{Cr}$ Thin-Film Depth Profile

$\mathrm{Cr} / \mathrm{CrO}_{2}$ Depth Profile

Boron Implant in Silicon Depth Profile

Urea

o-Bromobenzoic Acid

p-Fluorobenzoic Acid

m-Chlorobenzoic Acid

Nicotinic Acid (Calorimetry)

Urea (Calorimetry)

Low Alloy Steel

Low Alloy Steel

Low Alloy Steel

Low Alloy Steel

Low Alloy Steel

Low Alloy Steel

Low Alloy Steel

High Purity Iron

Hepes

Hepes Sodium Salt

Potassium Hydrogen Phthalate, pD

Potassium Dihydrogen Phosphate, $\mathrm{pD}$

2186II Disodium Hydrogen Phosphate, $\mathrm{pD}$

$2191 \mathrm{a}$

2192a

2201

2202

2203

2211

2213

Sodium Bicarbonate, $\mathrm{pD}$

Sodium Carbonate, pD

Sodium Chloride, $\mathrm{pNa} \& \mathrm{pCl}$

Potassium Chloride, pK \& pCl

Potassium Fluoride, $\mathrm{pF}$

Toluene $8 \mathrm{~mL}$

2,2,4-Trimethylpentane $25 \mathrm{~mL}$

Tin, Temp and Enthalpy of Fusion

2221a

2222

Date

107

May $82 \quad 108$

May $82 \quad 108$

Sep $80 \quad 108$

107

Feb $82 \quad 107$

Oct $84 \quad 106$

Oct $79 \quad 106$

May $80 \quad 106$

Jun $85 \quad 106$

Aug $87 \quad 41$

43

43

Feb $85 \quad 90$

$* \quad 124$
$* \quad 124$

Nov $84 \quad 124$

* 124

Al $585 \quad 123$

Aug $89 \quad 124$

Aug $89 \quad 124$

none 125

* 91

* 91

Aug $70 \quad 44$

Sep $70 \quad 44$

Jan 8244 


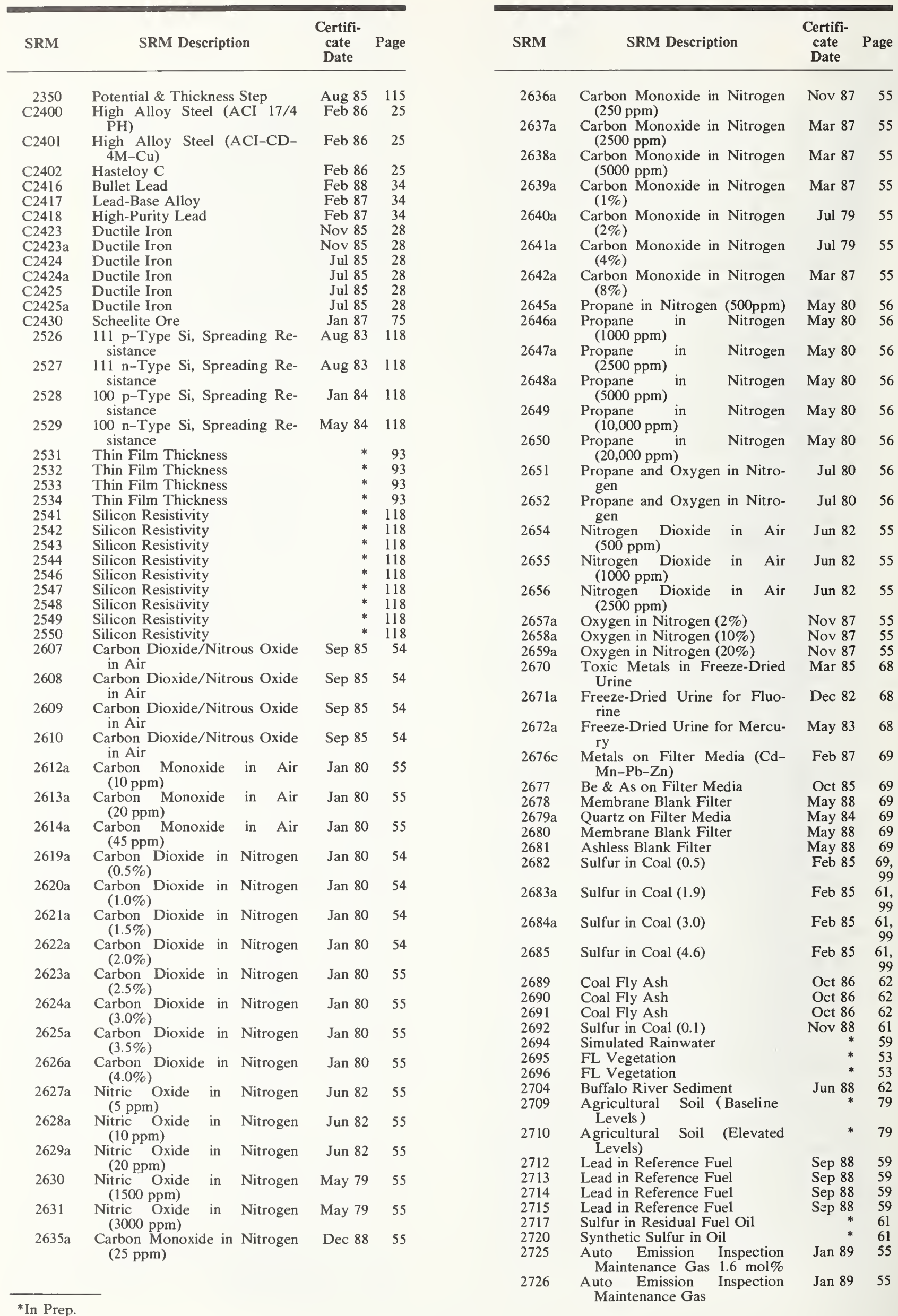


Auto Emission Inspection Maintenance Gas

2730 Hydrogen Sulfide in Nitrogen

2731 Hydrogen Sulfide in Nitrogen

3101 Aluminum Spectrometric Solution

3102

Antimony Spectrometric Solution

3103 Arsenic Spectrometric Solution

3104 Barium Spectrometric Solution

3105 Beryllium Spectrometric Solution

3106 Bismuth Spectrometric Solution

3107 Boron Spectrometric Solution

3108 Cadmium Spectrometric Solution

3109 Calcium Spectrometric Solution

3110 Cerium Spectrometric Solution

3111 Cesium Spectrometric Solution

3112 Chromium Spectrometric Solution

3113 Cobalt Spectrometric Solution

3114 Copper Spectrometric Solution

3115 Dysprosium Spectrometric Solution

$3116^{\circ}$ Erbium Spectrometric Solution

3117 Europium Spectrometric Solution

3118 Gadolinium Spectrometric Solution

3119 Gallium Spectrometric Solution

3120 Germanium Spectrometric Solution

3121 Gold Spectrometric Solution

3122 Hafnium Spectrometric Solution

3123 Holmium Spectrometric Solution

3124 Indium Spectrometric Solution

3125 Iridium Spectrometric Solution

3126 Iron Spectrometric Solution

3127 Lanthanum Spectrometric Solution

3128 Lead Spectrometric Solution

3129 Lithium Spectrometric Solution

3130 Lutetium Spectrometric Solution

3131 Magnesium Spectrometric Solution

3132 Manganese Spectrometric Solution

3133 Mercury Spectrometric Solution

3134 Molybdenum Spectrometric Solution

3135 Neodymium Spectrometric Solution

3136 Nickel Spectrometric Solution

3137 Niobium Spectrometric Solution

3138 Palladium Spectrometric Solution

3139 Phosphorus Spectrometric Solution

3140 Platinum Spectrometric Solution

3141 Potassium Spectrometric Solution

3142 Praseodymium Spectrometric Solution

3143 Rhenium Spectrometric Solution
Jan $89 \quad 55$

May $89 \quad 56$

May $89 \quad 56$

Nov $86 \quad 45$

Nov $86 \quad 45$

Nov $86 \quad 45$

Nov $86 \quad 45$

Nov $86 \quad 45$

Dec $86 \quad 45$

Dec 8645

Dec $86 \quad 45$

Nov $86 \quad 45$

Mar $87 \quad 45$

Feb $87 \quad 45$

Nov $86 \quad 45$

Dec $86 \quad 45$

Mar $87 \quad 45$

Mar $87 \quad 45$

Mar $87 \quad 45$

Mar $87 \quad 45$

Mar $87 \quad 45$

Mar $88 \quad 45$

Nov $86 \quad 45$

Apr $88 \quad 45$

Mar $87 \quad 45$

Dec $86 \quad 45$

Nov $86 \quad 45$

Mar $87 \quad 45$

Dec $86 \quad 45$

Nov $86 \quad 45$

Mar $87 \quad 45$

Nov 86

Dec 86

45

Dec $86 \quad 45$

Nov 86

45

Mar 87

Dec 86

Jan 88

45

Nov 86

Nov 86

45

45

45

Nov $86 \quad 45$

Nov $86 \quad 45$

Mar $87 \quad 45$

Jul $88 \quad 45$
Dec $86 \quad 45$
3144

3145

3146

3147

3148

3149

3150

3151

3152

3153

3154

3155

3156

3157

3158

3159

3160

3161

3162

3163

3164

3165

3166

3167

3168

3169

3171

3172

3173

3174

3181

3182

3183

3184

3185

3186

3191

3192

3193

3194

3195

3200

3216

3217

$4200 \mathrm{~B}$

$4201 \mathrm{~B}$

$4202 \mathrm{D}$

4203D

4207B

$4218 \mathrm{E}$

$4226 \mathrm{~B}$

$4233 \mathrm{C}$
Rhodium Spectrometric Solu-

Date

Rubidium Spectrometric Solution

Ruthenium Spectrometric Solution

Samarium Spectrometric Solution

Scandium Spectrometric Solution

Selenium Spectrometric Solution

Silicon Spectrometric Solution

Silver Spectrometric Solution

Sodium Spectrometric Solution

Strontium Spectrometric Solution

Sulfur Spectrometric Solution

Tantalum Spectrometric Solution

Tellurium Spectrometric Solution

Terbium Spectrometric Solution

Thallium Spectrometric Solution

Thorium Spectrometric Solution

Thulium Spectrometric Solution

Tin Spectrometric Solution

Titanium Spectrometric Solution

Tungsten Spectrometric Solution

Uranium Spectrometric Solution

Vanadium Spectrometric Solution

Ytterbium Spectrometric Solution

Yttrium Spectrometric Solution

Zinc Spectrometric Solution

Zirconium Spectrometric Solution

Multielement Mix A Solution

Multielement Mix B Solution

Multielement Mix C Solution

Multielement Mix D Solution

Sulfate Anion Solution

Chloride Anion Solution

Fluoride Anion Solution

Bromide Anion Solution

Nitrate Anion Solution

Phosphate Anion Solution

Electrolytic Conductance

Electrolytic Conductance

Electrolytic Conductance

Aqueous Electrolytic Conductance

Aqueous Electrolytic Conductance

Secondary Standard Magnetic Tape (Computer Amplitude Ref)

Secondary Standard Magnetic Tape Cartridge (Computer Amplitude Ref)

Secondary Standard Magnetic Tape Cartridge-High Density (C A Ref)

Cesium-137/Barium-137m Point Source

Niobium-94 Gamma-ray

Cadmium-109-Silver-109m

Cobalt-60 Point Source

Cesium-137/Barium-137m Point Source

Europium-152 Point Source

Nickel-63 Solution

Cesium-137 Burn-up Standard

Nov $86 \quad 45$

Mar $87 \quad 46$

Mar $87 \quad 46$

Dec $86 \quad 46$

Dec 8646

Dec $86 \quad 46$

Nov $86 \quad 46$

Nov $86 \quad 46$

Aug $87 \quad 46$

Jan $88 \quad 46$

Aug $87 \quad 46$

$\operatorname{Mar} 87 \quad 46$

Dec $86 \quad 46$

Jan $88 \quad 46$

Mar $87 \quad 46$

Nov $86 \quad 46$ 


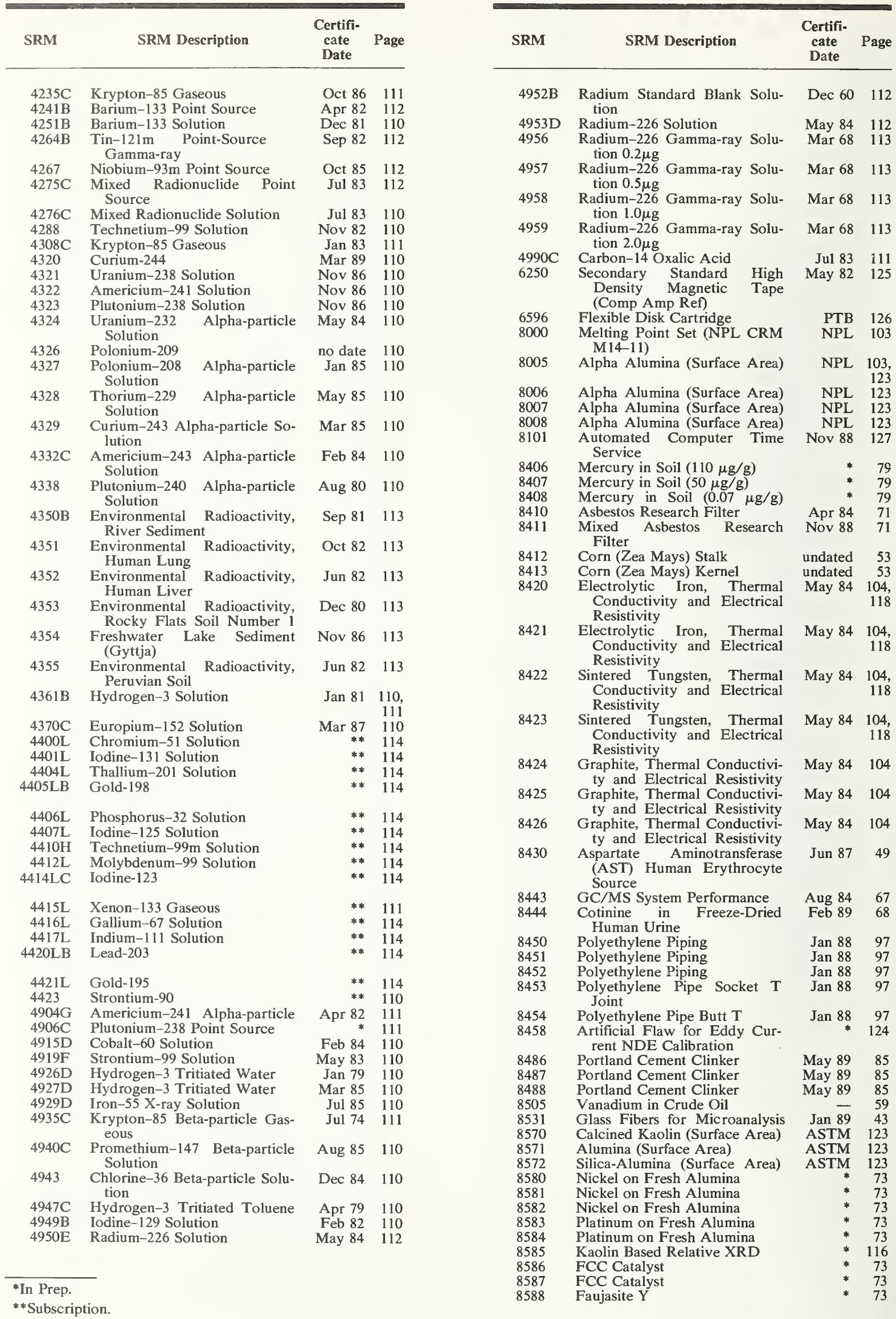




\begin{tabular}{|c|c|c|c|}
\hline SRM & SRM Description & $\begin{array}{l}\text { Certifi- } \\
\text { cate } \\
\text { Date }\end{array}$ & Page \\
\hline 8589 & FCC Catalyst & * & 73 \\
\hline 8590 & High Sulfur Gas Oil Feed & * & 61 \\
\hline 9111 & SRM Compilation & Nov 87 & 132 \\
\hline 9529 & Flexible Disk Cartridge & PTB & 126 \\
\hline GM 754 & $\begin{array}{l}\text { ICTA Polystyrene (DTA \& } \\
\text { DSC) }\end{array}$ & ICTA & 101 \\
\hline GM 757 & $\begin{array}{l}\text { ICTA Temp Set Below } 350 \mathrm{~K} \\
\text { (DTA\&DSC) }\end{array}$ & ICTA & 101 \\
\hline
\end{tabular}

\begin{tabular}{|c|c|c|c|}
\hline SRM & SRM Description & $\begin{array}{c}\text { Certifi- } \\
\text { cate } \\
\text { Date }\end{array}$ & Page \\
\hline GM 758 & $\begin{array}{l}\text { ICTA Temp Set } 125-435 \text { C } \\
\text { (DTA\&DSC) }\end{array}$ & ICTA & 101 \\
\hline GM 759 & $\begin{array}{l}\text { ICTA Temp Set 295-675 C } \\
\text { (DTA\&DSC) }\end{array}$ & ICTA & 101 \\
\hline GM 760 & $\begin{array}{l}\text { ICTA Temp Set } 570-940 \mathrm{C} \\
\text { (DTA\&DSC) }\end{array}$ & ICTA & 101 \\
\hline GM 761 & ICTA Thermogravimetry Set & ICTA & 101 \\
\hline RM 1R & Aluminum, Ultra-Purity (rod) & & 40 \\
\hline RM 50 & Albacore Tuna & May 77 & 51 \\
\hline
\end{tabular}





\section{Alphabetical Index}

ABRASIVE WEAR, 115

Tool Steel

ABSORBANCE, see Spectrophotometry

ACETANILIDE, 44

Microchemical

ACIDIMETRIC, 44

(See also, Spectrometry)

Benzoic Acid

Boric Acid

Potassium Hydrogen Phthalate

ACID RAIN, 59

ACOUSTIC EMISSION TRANSDUCER, 97

ADHESION TESTING TAPE, 128

Linerboard

AGRICULTURE, 53

(See also, Food and Beverage)

Ammonium Dihydrogen Phosphate

(Fertilizer), 74

Bovine Liver, 51

Brewers Yeast, 51

Citrus Leaves, 53

Coconut Oil, 52

Corn, 53

Cystine, 44

Dextrose, 44, 108

Mercury in Water, 59

Milk, Non-fat Powdered, 51

Oyster Tissue, 51

Phosphate Rock (Fertilizer), 74, 77

Pine Needles, 53

Potassium Dihydrogen Phosphate (Fertilizer), 74

Potassium Nitrate (Fertilizer), 74

Rice Flour, 51

Sucrose, 44, 108

Tomato Leaves, 53

Trace Elements in Water, 62

Tuna, Albacore, 51

Wheat Flour, 51

Wine, Stabilized, 52

AIR PARTICULATES, 62

AIR POLLUTION, 54

ALCOHOL

Alcohol in Ref. Fuel, 60

Ethanol in Ref. Fuel, 60

Methanol-Butanol in Ref. Fuel, 60

Methanol in Ref. Fuel, 60

Stabilized Wine, 52

ALLOYS

(See also individual metals)

Ferrous, 15

High Temperature, 30

Nonferrous, 30

Steelmaking, 26

ALPHA QUARTZ, 70

ALUMINA

Melting Point, 102

Reduction Grade, 77
Surface Area, 103, 123

ALUMINUM

Alloys-Composition, 30

Conductivity, 104, 118

First Surface Mirror, 107

Freezing Point, 102

High-Purity, 30, 40

Magnetic Susceptibility, 105

Metallo-Organic, 72

Mirrors, Specular Reflectance, 107

Residual Resistivity Ratio, 119

Spectrometric Solution, 45

Specular Reflectance Mirrors, 107

Ultra-Purity, 40

AMERICIUM, 109-114

Radioactivity

AMMONIUM DIHYDROGEN

PHOSPHATE, 74

Fertilizer

ANALYZED GASES, see Gases

ANGIOTENSIN I (Human), 49, 50

Clinical

ANISIC ACID, 44

Microchemical

ANION SOLUTIONS, 48

Bromide

Chloride

Fluoride

Nitrate

Phosphate

Sulfate

ANTICONVULSANT DRUG LEVEL

ASSAY, 49

Clinical

ANTIEPILEPSY DRUG LEVEL ASSAY, 49

Clinical

ANTIMONY,

Radioactivity, 110

Spectrometric Solution, 45

ARGILLACEOUS LIMESTONE, 79

ARSENIC

-on Filter Media, 68

Spectrometric Solution, 45

-Trioxide, Reductometric, 44

ASBESTOS, 71

Bulk-Common, Uncommon

Chrysotile Fibers

Research Filter

ATOMIC ABSORPTION, see Spectrometric

Solutions

AUSTENITE, 115

in Ferrite 


\section{BARIUM}

Metallo-Organic, 72

Radioactivity, 109-114

Spectrometric Solution, 45

BASALT, 80

Rock

BASIMETRIC, 44

BAUXITE, 77

BEARING METAL, 34

Lead Alloy

BENZOIC ACID

Acidimetric, 44

Calorimetric, 99

BERYLLIUM

Copper Alloys, 32

-on Filter Media, 69

Spectrometric Solution, 45

BET SURFACE AREA, 123

BEVERAGE, see Food and Beverage

BILIRUBIN, 49

Clinical

BIOLOGICAL, 51-53

Agricultural

Ethanol Solutions

Food and Beverage

BIPHENYL, 100

Enthalpy

BISMUTH

Spectrometric Solution, 45

BLACK PORCELAIN ENAMEL, 108 Reflectance

BLOOD GAS, 49, 54 Analyzed Gases

BORATE ORE, 77

BORON

Boric Acid (Assay \& Isotopic), 44, 88

Boric Acid, Enriched B-10, 88

Depth Profile, 91

Implant in Silicon, 91

Spectrometric Solution, 45

BOTANICAL, see Biological

BOVINE

-Liver, 51

-Serum, Inorganic Constituents, 49

BOVINE SERUM ALBUMIN, 49

Clinical

BRASS, see Copper Alloys

BREWERS YEAST, 51

BROMIDE

Anion Solution, 48

BROMINE, 88

Isotopic

o-Bromobenzoic Acid

BRONZE, see Copper Alloys

BURNT REFRACTORIES, 79

BUTYL, 121

Rubber

\section{CALCIUM}

Carbonate (Clinical), 49

Molybdate, 26

Spectrometric Solution, 45

CALORIMETRY

(See also, Fuels \& Fossil Fuels)

Combustion, 99

Enthalpy, 97, 98, 100

Heat Capacity, 99, 100

Heating Value, 99, 100

Heat Source, 99

Solution, 99

CARBIDES, 81

Cemented, 82

Silicon

Tungsten

CARBON

(See also, Rubber Compounding Materials)

Carbon Dioxide, 54

Carbon Monoxide, 55

Radioactivity, 109-114

Steels, 15

CAST IRON, 27

CAST STEEL, 28

CATALYST CHARACTERIZATION

MATERIALS, 73

CATALYST PACKAGE FOR LUBRICANT

OXIDATION, 73

CEMENTS

Portland, Composition, 84

Portland, Fineness, 122

CENTERLINE DRAWINGS FOR OCR, 126

CERAMIC MATERIALS

Carbides, 81

Glasses, 83

Minerals, 79

Reflectance, 107

Refractories, 80

CERIUM

Spectrometric Solution, 45

CESIUM

Radioactivity, 110

Spectrometric Solution, 45

CHANNEL BLACK, 121

Rubber Compounding Material

CHARPY V-NOTCH TEST BLOCKS, 124

CHEMICAL

High Purity Metals, 40

Microchemical, 44

Primary, Working, and Secondary, 44

CHLORIDE

Anion Solution, 48

CHLORINE

m-Chlorobenzoic Acid, 44

in Lube Base Oil, 72

Isotopic, 88

Radioactivity, 109-114

CHOLESTEROL, 49

-and Fat Soluble Vitamins in Coconut Oil

Clinical

in Egg Powder

in Human Serum

CHROMIUM

Chrome Refractory, 79

$\mathrm{Cr} / \mathrm{CrO}_{2}$ Depth Profile, 91

Isotopic, 88 
Metallo-Organic, 72

Radioactivity, 109-114

Spectrometric Solution, 45

Steel, 16

CHRYSOTILE, 71

Asbestos

CITRUS LEAVES, 53

CLAYS, 78

Brick

Flint

Plastic

CLINICAL LABORATORY, 49

Angiotensin I (Human)

Anticonvulsant Drug Level Assay

Antiepilepsy Drug Level Assay

AST Human Erythrocyte Source

Bilirubin

Blood Gases

Bovine Serum Albumin

Bovine Serum, Inorganic Constituents

Calcium Carbonate

Cholesterol

Cortisol (Hydrocortisol)

Creatinine

D-Glucose (Dextrose)

Human Serum

4-Hydroxy-3-methoxy-D1-mandelic Acid (VMA)

Iron Metal

Lead Nitrate

Lithium Carbonate

Magnesium Gluconate Dihydrate

D-Mannitol

4-Nitrophenol

Potassium Chloride

Sodium Chloride

Sodium Pyruvate

Tris(hydroxymethyl)aminomethane, $\mathrm{pH}$

Tris(hydroxymethyl)aminomethane hydrochloride, $\mathrm{pH}$

Urea

Uric Acid

COAL

Calorimetric, 61, 99

Sulfur in, 61

Trace Elements in, 62

Trace Mercury in, 59, 62

COAL FLY ASH, 62

Trace Elements in

COATING THICKNESS, 92

$\mathrm{Cu}-\mathrm{Cr}$ Coating (nonmagnetic) on Steel

Nickel (magnetic) on Steel

Solder Thickness Standards, 92

COATING WEIGHT, 93

Gold on Nickel

Ultra-Thin Gold on Nickel

COBALT

Metallo-Organic, 72

Radioactivity, 109-114

Spectrometric Solution, 45

COCAINE METABOLYTE, 68

COCONUT OIL, 52

COD LIVER OIL, 63

Co-Cr-Mo ALLOY, 116

Pitting/Crevice Corrosion

COLOR, 125

Centroid Color Charts
COMPUTER, MAGNETIC STORAGE

MEDIA, 125

CONDUCTANCE (AQUEOUS), 119

CONDUCTIVITY,

Aluminum, 119

Electrical, 118

Glass, 94

Graphite, 104

Iron, 104, 118

Silicon, 118

Steel, 104, 118

Thermal, 104

Tungsten, 104, 118

COPPER

Alloys, 31

Benchmarks (Unalloyed Cu), 33

Brass, 32

Bronze, 32

Freezing Point, 102

Gilding Metal, 32

Heat Capacity Test Specimen, 100

Isotopic, 88

Metallo-Organic, 72

Microprobe, 41, 42

Ores, 75

Spectrometric Solution, 45

CORN, 53

CORROSION, 115

Implants

Pitting or Crevice Corrosion

Potential and Thickness Step Test

CORTISOL, 49

Clinical

COTININE IN URINE, 68

CREATININE, 49

Clinical

CRUDE OIL, 59

CURIUM, 109-114

Radioactivity

CYSTINE, 44

Microchemical

\section{DENSITY}

Liquids, 96

Neutral Filters, 106

Neutron, 87

Photographic, 125

Silicon, 96

Smoke, 128

X-ray, 125

DEPTH PROFILE, 91

Boron Implant in Silicon

$\mathrm{Cr} / \mathrm{CrO}_{2}$ Thin-Film

$\mathrm{Ni} / \mathrm{Cr}$ Thin-Film

DEXTROSE, see D-Glucose

DIDYMIUM

Wavelength, 106 
DIFFERENTIAL SCANNING

CALORIMETRY, 100

DIFFERENTIAL THERMAL ANALYSIS, 101

DIFFRACTION, X-RAY, 116

DIOXIN' IN ISOOCTANE, 63, 67

DISODIUM HYDROGEN PHOSPHATE, 89 $\mathrm{pH}, \mathrm{pD}$

DOLOMITIC LIMESTONE, 79

DOSIMETRY, 87

DRUG LEVEL ASSAY, see Clinical

DRUGS OF ABUSE, IN URINE, 68

DSC, see Heat

DTA, see Heat

DUST

Urban, 63, 64

DYE PENETRANT CRACK BLOCK, 123

DYSPROSIUM

Spectrometric Solution, 45

EDDY CURRENT

Aluminum, 119

Artificial Flaw, 124

Silicon, 118

Test Block, 123

ELECTRICAL CONDUCTIVITY, 104, 118

ELECTRICAL RESISTIVITY, 104, 118

Graphite

Iron

"RRR" Set

Silicon

Tungsten

ELECTROLYTIC CONDUCTANCE, 119

ELECTRONIC AND MAGNETIC ALLOY, 35

ELLIPSOMETRY THIN FILM, 93

ENGINEERING MATERIALS, 121

ENTHALPY, see Heat

ENVIRONMENTAL

Diesel Particulate Matter, 63, 64

Estuarine Sediment, 62

Radioactivity, Natural Matrices, 113

River Sediment, 62

Urban Particulate Matter, 62

ENZYME, 49

Aspartate Aminotransferase Human

ERBIUM

Erythrocyte Source

Spectrometric Solution, 45

ESTAURINE SEDIMENT, 62

ETHANOL SOLUTIONS, 60

Ethanol-Water Solutions

EUROPIUM

Radioactivity, 109-114

Spectrometric Solution, 45
Soda

FERRITE, 115

FERRO-ALLOYS, see Steelmaking Alloys

FERTILIZERS, 74

Ammonium Dihydrogen Phosphate

Phosphate Rock

Potassium Dihydrogen Phosphate

Potassium Nitrate

FILTER MEDIA, 69

$\mathrm{Be} \&$ As on

Metals on (Cd-Mn- $\mathrm{Pb}-\mathrm{Zn})$

Quartz on

Toxic Metals on

FILTERS, 106

FINENESS, 122

Portland Cement

FIRE RESEARCH, 128

FISSION TRACK, 87

FLAMMABILITY, 128

FLEXIBLE DISK CARTRIDGE

Magnetic Computer Storage Media, 125

FLOORING RADIANT PANEL, 128

FLOUR

Rice, 51

Wheat, 51

FLUORESCENCE

Glass, 116

Quinine Sulfate Dihydrate, 106

$\mathrm{X}$-ray, Glass Target, 116

FLUORINE, 68

in Freeze-Dried Urine

p-Fluorobenzoic Acid

FLUORSPAR, 75

Customs Grade

High Grade

FLY ASH, COAL, 62

Lime

Trace Elements

FOOD AND BEVERAGE

(See also, Agriculture)

Coconut Oil, 52

Cod Liver Oil, 52

Corn, 53

Liver, Bovine, 51

Non-Fat Milk Powder, 51

Oyster Tissue, 51

Rice Flour, 51

Tuna, Albacore, 51

Wheat Flour, 51

Wine, Stabilized, 52

Yeast, Brewers, 51

FOSSIL FUELS

(See also, Fuels)

Chlorine in Lube Base Oil, 72

Heating Values, 99

Lime in Coal Fly Ash, 62

Nitrogen in Lube Base Oil, 72

Sulfur in Coal, 61

Sulfur in Distillate Fuel Oil, 61

Sulfur in Kerosine, 61

Sulfur in Residual Fuel Oil, 61

Trace Elements in Coal, 62

Trace Elements in Coal Fly Ash, 62

Trace Elements in Fuel Oil, 62

Trace Mercury in Coal, 59

$\mathrm{V}$ and Ni in Residual Fuel Oil, 59 
FREEZING POINT, 102

(See also, Heat \& Melting Point)

Aluminum

Copper

Lead

Mercury

Tin

Zinc

FUELS

(See also, Calorimetry)

Alcohol in Reference Fuel (Gasoline), 60

Ethanol in Reference Fuel (Gasoline), 60

Heating Values, 99

n-Heptane, 118

Isooctane, 118

Lead in Reference Fuel (Gasoline), 59

Lime in Coal Fly Ash, 62

Methanol-Butanol in Reference Fuel (Gasoline), 60

Methanol in Reference Fuel (Gasoline), 60

Reference Fuels, 118

Synthetic Refuse-Derived Fuel

(Gasoline), 99

Sulfur in Coal, 61

Sulfur in Distillate Fuel Oil, 61

Sulfur in Kerosene, 61

Sulfur in Residual Fuel Oil, 61

Synthetic Sulfur in Oil, 61

Trace Elements in Coal, 62

Trace Elements in Coal Fly Ash, 62

Trace Elements in Fuel Oil, 62

Trace Mercury in Coal, 59

$\mathrm{V}$ and $\mathrm{Ni}$ in Residual Fuel Oil, 59

FUMED SILICA BOARD, 104

\section{GADOLINIUM}

Spectrometric Solution, 45

GALLIUM

Isotopic, 44

Melting Point, 102

Radioactivity, 109-114

Spectrometric Solution, 45

GAS CHROMATOGRAPHY, 67

GC/MS System Performance

GAS FURNACE BLACK, 121

GASES, 54

Aliphatic Organic

Aromatic Organic

Benzene in Nitrogen

Blood Gases

Carbon Dioxide in Air

Carbon Dioxide in Nitrogen

Carbon Dioxide in Nitrogen, Blood Gas

Carbon Dioxide and Oxygen in Nitrogen, Blood Gas

Carbon Monoxide in Air

Carbon Monoxide in Nitrogen

Methane and Propane in Air

Methane in Air

Nitric Oxide in Nitrogen

Nitrogen Dioxide in Air

Nitrogen Dioxide Permeation Device
Organic

Oxygen in Nitrogen

Propane and Oxygen in Nitrogen

Propane in Air

Propane in Nitrogen

Sulfur Dioxide in Nitrogen

Sulfur Dioxide Permeation Tubes

Tetrachloroethylene in Nitrogen

Tetrachloroethylene Permeation Device

GASES IN METALS, 39

Hydrogen

Oxygen

GASOLINE, see Fuels

GAS TRANSMISSION, 117

Polyester Film

GEOLOGICAL, 79

GERMANIUM

Spectrometric Solution, 45

GLASS BEADS, see Sizing

GLASSES, CHEMICAL COMPOSITION

Glass, 83

Microanalytical, 41

GLASSES, PHYSICAL PROPERTIES

Dielectric Constant, 94

Electrical Resistance, 94

Fixed Points, 95

Fluorescence Source, 116

Leaching Resistance, 94

Liquidus Temperature, 95

Stress Optical Coefficient, 95

Thermal Expansion, 104

Viscosity, 94

GLASS SAND, 79

GLASS SPHERES, see Sizing

D-GLUCOSE (Dextrose)

Clinical, 49

GOLD

Primary Chemical, 44

Coating Weight, 93

High Purity, 40

Microprobe, 41

Mirror (Reflectance), 107

Radioactivity, 109-114

Reflectance (Specular), 107

Spectrometric Solution, 45

Vapor Pressure, 102

GRAPHITE, 104

Thermal Conductivity

HAFNIUM, 38

in Zircaloy

in Zirconium

Spectrometric Solution, 45

HARDNESS, 96

Microhardness

HASTELOY, 25

HEAT

(See also, Fuels)

Calorimetric, 99

Differential Scanning Calorimetry, 100

Differential Thermal Analysis, 101

Enthalpy, 100

Freezing Points, 102 
Heat Capacity, 100

Heat Source, 99

Heating Values, 99

Melting Points, 102

Superconducting Fixed Points, 101

Thermal Conductivity, 104

Thermal Expansion, 104

Thermocouple Material, 103

Thermogravimetry, 101

Vapor Pressure, 102

HEPES (and salts), 89

n-HEPTANE, 118

HIGH ALLOY STEEL, 18

HIGH PURITY METALS, 18

(See also, Spectrometric Solutions)

Aluminum (See A1 Alloys)

Cadmium (See Vapor Pressure)

Copper (See Cu Benchmarks)

Gallium (See Stable Isotopes)

Gold

Iron (See Iron, Electrolytic)

Lead (See Freezing Points)

Magnesium (See Stable Isotopes)

Mercury (See Freezing Points)

Nickel (See Magnetic Moment)

Platinum

Rhenium (See Stable Isotopes)

Selenium

Silicon (See X-ray Powder Diffraction)

Silver (See Vapor Pressure)

Tin (See Freezing Points)

Titanium (See Ti Alloys)

Tungsten (See Thermal Conductivity)

Zinc

Zirconium (See Zr Alloys)

HIGH TEMPERATURE ALLOYS, 25

HOLMIUM

Oxide, Wavelength, 106

Wavelength, 106

Spectrometric Solution, 45

HUMAN

Liver (Radioactivity), 113

Lung (Radioactivity), 113

Serum (Clinical), 49, 50

HUMAN SERUM, 49, 50

AST Enzyme

Cholesterol in

Clinical

PCB's in

HYDROGEN

Gases in Metals, 39

Radioactivity, 109-114

4-HYDROXY-3-METHOXY-DL-

MANDELIC ACID (VMA), 49

\section{IMAGE QUALITY INDICATOR}

Radiographic, 125

IMPLANTS, see Corrosion

INDIUM

Melting Point, 102

Radioactivity, 109-114

Spectrometric Solution, 45
INDUSTRIAL HYGIENE, 68

INFRARED, NEAR, 107

Reflectance

Wavelength

INSTRUMENT PERFORMANCE

Atomic Absorption Spectrophotometer, 45

Beta-Backscatter Gages, 93

Calorimetry, 99

Conductivity Meters, 119

Coordinate Measuring Machines, 123

Differential Scanning Calorimetry, 100

Differential Thermal Analysis, 101

Dilatometers, 104

Electron Microprobe, 41, 42

Ellipsometers, 93

Gas Analysis, 54

GC/MS, 67

Hardness, Micro-, 96

Optical Emission Spectrometers, 15-40

Optical Microscopes, 91

Particle Counters, 122

pH Meters, 89

Polarimetry, 108

Profilometers, 124

Radioactivity Counting Systems, 109-114

Reflectometers, 107

Sacchrimeters, 108

SEM's, 90

Spectrophotometers, 106

Surface Analyzers, 123

Thermometers, 103

Turbidimeters, 122

Viscometers, 94, 95

X-ray Diffraction, 116

$\mathrm{X}$-ray Fluorescence, 116

IODINE, 109-114

Radioactivity

ION ACTIVITY, 90

Potassium Chloride

Potassium Fluoride

Sodium Chloride

IRIDIUM IRON

Spectrometric Solution, 45

Alloy Cast, 27, 28

Blast Furnace, 28

Cast, 27, 28

Clinical, 49, 50

Ductile Cast, 27, 28

Electrical Resistivity, 104, 118

Electrolytic, 17

Gray Cast, 27

High Alloy White Cast, 28

Metallo-Organic, 72

Nodular Cast, 27

Radioactivity, 109-114

Spectrometric Solution, 45

Thermal Conductivity, 104

White, 27, 28

ISOBUTYLENE-ISOPRENE, 121

Rubber

ISOTOPIC (STABLE), 88

Boron

Bromine

Chlorine

Chromium 
Copper

Gallium

Lead, Common

Lead, Equal-Atom

Lead, Radiogenic

Magnesium

Nickel

Potassium

Rhenium

Rubidium

Silicon

Silver

Strontium

Thallium

KAOLIN, 123

Surface Area

KEROSINE, 61

Sulfur in

KNOOP, 96

Microhardness

KRYPTON, 109-114

Radioactivity

\section{LANTHANUM}

Spectrometric Solution, 45

LEAD

Alloys, 34

Base Material, 34

-Based Paint, Powdered, 59

Freezing Point, 102

-in Reference Fuels, 59

Isotopic, 88

-on Filter Media, 69

Metallo-Organic, 72

Nitrate (Clinical), 49

Radioactivity, 109-114

Spectrometric Solution, 45

LEAVES, 53

Citrus

Pine Needles

Tomato

LIMESTONE, 79

Argillaceous

Dolomitic

LINERBOARD, 128

Tape Adhesion Testing

LINEWIDTH MEASUREMENT, 91

LITHIUM

Carbonate (Clinical), 49

Metallo-Organic, 72

Ores, 75

Spectrometric Solution, 45

LIVER

Bovine, Biological, 51

Human, Radioactivity, 113
LUBRICANT OXIDATION PACKAGE, 73

LUBRICATING OIL

Chlorine in, 72

$\mathrm{Ni}$ and $\mathrm{V}$ in, 59

Nitrogen in, 72

Sulfur in, 72

Wear-Metals in, 73

LUNG, 113

Human, Radioactivity

LUTETIUM

Spectrometric Solution, 45

MAGNESIUM

Clinical, 45

Isotopic, 88

Metallo-Organic, 72

Spectrometric Solution, 45

MAGNETIC COMPUTER STORAGE

MEDIA, 125

Cartridge: High-Density Tape

Cartridge: Tape

Cassette: Tape

Flexible Disk

Reel: High-Density Tape

Reel: Tape

MAGNETIC MOMENT, 105

Nickel

MAGNETIC SUSCEPTIBILITY, 105

Aluminum

Manganese Fluoride

Palladium

MANGANESE

-on Filter Media, 69

Spectrometric Solution, 45

D-MANNITOL, 49

Clinical

MARIAJUANA METABOLITE (THC IN FREEZE DRIED URINE), 68

MARINE CHEMISTRY

(See also, Environmental, Sediment, \& Water Analysis)

Albacore Tuna, 51

Anion Solution, 48

Estuarine Sediment, 62

Limestone, 79

Oyster Tissue, 51

PCB's in Sediment, 63

Spectrometric Solution, 45

MATERIALS ON FILTER MEDIA, 69

Arsenic

Beryllium

Cadmium

Lead

Manganese

Quartz

Zinc

MELTING POINT, 102

(See also, Heat \& Freezing Point)

Alumina

Gallium

Indium

Rubidium

Succinonitrile 
MERCAPTOBENZOTHIAZOLE, 121

\section{MERCURY}

Freezing Point, 102

-in Coal, 59

-in Freeze-Dried Urine, 68

-in Soil, 79

-in Water, 59

Radioactivity, 109-114

Spectrometric Solution, 45

METALLO-ORGANIC COMPOUNDS, 72

Aluminum

Barium

Cadium

Calcium

Chromium

Copper

Iron

Lead

Lithium

Magnesium

Nickel

Phosphorus

Silicon

Silver

Sodium

Strontium

Tin

Vanadium

Zinc

METALLURGICAL, 115

Austenite in Ferrite

Iron Carbide in Ferrite

METALS ON FILTER MEDIA, 69

Arsenic

Beryllium

Cadmium

Lead

Manganese

Zinc

METHANE, 55

METROLOGY

Depth Profile, 91

Optical Linewidth, 91

Particle Size, 122

SEM Magnification, 90

Socketed Ball Bar, 123

MICROANAL YTICAL, 41, 42

$\mathrm{Fe}-\mathrm{Cr}-\mathrm{Ni}$ Alloy

Gold-Copper

Gold-Silver

Iron-Silicon

Mg-Si-Ca-Fe

Mineral Glasses

Thin Film

Tungsten-Molybdenum

MICROCHEMICAL, 44

Acetanilide

Anisic Acid

o-Bromobenzoic Acid

m-Chlorobenzoic Acid

Cystine

p-Fluorobenzoic Acid

Nicotinic Acid

Urea

MICROHARDNESS, 96

MICROSCOPY, 91, 122

Optical
Scannaing Election

Knoop

Vickers

MICROPROBE, see Microanalytical

MICROSPHERE, 122

MILK, NON-FAT POWDERED, 51

MINERALS, 79

MOLECULAR WEIGHT-MELT FLOW, 97 , 98

Polymers

MOLYBDENUM

Concentrate, 75

Heat Capacity, 97, 98, 100

Radioactivity, 109-114

Spectrometric Solution, 45

NEODYMIUM

Spectrometric Solution, 45

NICKEL

Alloys, 35

Isotopic, 88

Magnetic Moment, 105

Metallo-Organic, 72

Oxides, 36

Radioactivity, 109-114

Spectrometric Solution, 45

NICOTINIC ACID, 44

Microchemical

NIOBIUM, 109-114

Radioactivity

Spectrometric Solution, 45

NIST TIME SOFTWARE, 127

NITRATE, 48

Anion Solution

NITRIC OXIDE, 55

NITROGEN

in Lubricating Base Oil, 72

Nitrogen Dioxide, 55

NITROUS OXIDE, 55

4-NITROPHENOL, 49

Clinical

NONDESTRUCTIVE EVALUATION (NDE), 123

Dye Penetrant Test Blocks

NONFERROUS ALLOYS, 30

N-TERTIARY-BUTYL-2-

BENZOTHIAZOLESULFENAMIDE, 121

Rubber Compounding Material

NUCLEAR MATERIALS, 87

Fission Track Glass

Neutron Density Monitor Wire

NUTRITION, see Food and Beverage, and Diet

OBSIDIAN ROCK, 80

OIL

Base Oil, Wear Metals in, 73

Chlorine in Lube Base Oil, 72 
Distillate (Diesel) Fuel Oil (Sulfur), 61 Nitrogen in Lube Base Oil, 72

Petroleum Crude Oil (Organics), 63, 64

Residual Fuel Oil (Sulfur), 61

Residual Fuel Oil (Trace Elements), 62

Residual Fuel Oil (V and Ni), 61

Sulfur in Lube Base Oil, 72

OIL FURNACE BLACK, 121

OPAL GLASS

Composition, 83

Reflectance, 107

OPTICAL

(See also, Reflectance and

Spectrophotometry)

Centerline Drawings for OCR, 126

Linewidth Measurement, 91

ORES, 75

Alumina

Bauxites

Copper

Fluorspar

Iron

Lithium

Manganese

Molybdenum

Phosphate Rock

Reduced Iron Oxide

Rutile

Scheelite

Tungsten

ORGANIC CONSTITUENTS, 66, 67

Chlorinated Biphenyls

Chlorinated Pesticides

Dinitropyrene Isomers

Dioxin in Isooctane

Generator Columns for PAH's

Halocarbons for Water Analysis

-in Cod Liver Oil

-in Marine Sediment

I-Nitropyrene in Methylene Chloride

Isotopically Labeled and Unlabeled

Priority Pollutants

Nitrated Polycyclic Aromatic

Hydrocarbons

Organics in Shale Oil

PAH's (from Coal Tar)

Petroleum Crude Oil

Polychlorinated Biphenyls in Human

Serum

Polychlorinated Biphenyls in Oil

Polychlorinated Biphenyls in Sediments

Priority Pollutant Phenols in Methanol

Priority Pollutant PAH's

Urban Dust

OXALIC ACID, 111

Radioactivity

OXYGEN, 55

Analyzed Gas

OYSTER TISSUE, 51

\section{PACKAGE}

Catalyst Package for Lubricant

Oxidation, 73
PAINT

Lead-Based, 59

PALLADIUM

Spectrometric Solution, 45

PARTICLE SIZE, 122

PARTICULATES

Metals on Filter Media, 69

Urban Dust/Organics, 63, 64

pD, 89

Urban, Trace Elements, 62

Disodium Hydrogen Phosphate

Hepes

Potassium Dihydrogen Phosphate

Potassium Hydrogen Phthalate

Sodium Bicarbonate

Sodium Carbonate

PERMEATION DEVICES, 57

PERUVIAN SOIL, 113

Radioactivity

PESTICIDES, 65

pH, 89

Disodium Hydrogen Phosphate

Potassium Tetroxalate

Sodium Bicarbonate

Sodium Carbonate

Sodium Tetraborate Decahydrate (Borax)

Tris(hydroxymethyl)aminomethane

Tris(hydroxymethyl)aminomethane hydrochloride

PHOSPHATE

Anion Solution, 48

PHOSPHORUS

Metallo-Organic, 72

Phosphate Rock, 74

Radioactivity, 109-114

Spectrometric Solution, 45

PHOTOGRAPHIC, 125

Photographic Step Tablet (0-4)

Microcopy Resolution Test Charts

PINE NEEDLES, 53

PLASTIC, see Polymer

PLASTIC PIPE, 97

PLATINUM

Doped, 40

High Purity, 40

Spectrometric Solution, 45

Thermoelement, 103

PLUTONIUM

Radioactivity, 109-114

POLLUTANTS

Atmospheric Gases, 54-56

Dust, 63, 64

Inorganics, 62

Organics, 68

Rainwater, Simulated, 59

Sediments, 62

Spectrometric Solution, 45

POLONIUM, 109-114

Radioactivity

POLYCHLORINATED BIPHENYLS (PCB)

in Human Serum, 49, 67

in Oil, 63, 65

in Sediment, 63

POLYESTER FILM, 117

POLYETHYLENE, 97

Molecular Weight 
POLYISOBUTYLENE, 98

Rheology

POLYMER

Particle Size, 122

Polyethylene (Molecular Weight), 97

Polyisobutylene (Rheology), 98

Poly(methyl methacrylate), 97, 98

Polystyrene (Molecular Weight), 97

POLY(METHYL METHACRYLATE), 97, 98

POLYSTYRENE

Differential Thermal Analysis, 101

Molecular Weight, 97, 98, 100

Particle Size, 122

POTASSIUM

-Chloride (Calorimetric), 99

-Chloride (Clinical), 49

-Chloride (Electrolytic Conductance), 119

-Chloride (Isotopic), 88

-Chloride (Primary), 44

-Dichromate (Primary), 44

-Dichromate (Spectrophotometry), 106

-Dihydrogen Phosphate (Fertilizer), 74

-Dihydrogen Phosphate ( $\mathrm{pH}), 89$

-Hydrogen Tartrate $(\mathrm{pH}), 89$

-Hydrogen Phthalate $(\mathrm{pH}), 89$

-Hydrogen Phthalate (Primary), 44

-Iodide (Stray Light), 106

-Nitrate (Enthalpy), 100

-Nitrate (Fertilizer), 74

Spectrometric Solution, 45

-Tetroxalate $(\mathrm{pH}), 89$

POTENTIAL AND THICKNESS STEP

TEST, 115

POWDERED LEAD-BASED PAINT, 59

PRAESODYMIUM

Spectrometric Solution, 45

PRIMARY CHEMICALS, 44

Arsenic Trioxide

Benzoic Acid

Boric Acid

Dextrose (D-glucose)

Potassium Chloride

Potassium Dichromate

Potassium Hydrogen Phthalate

Sodium Oxalate

Strontium Carbonate

Sucrose

Tris(hydroxymethyl)aminomethane

PRIORITY POLLUTANTS, see Organics

PROMETHIUM, 109-114

Radioactivity

QUARTZ, 70

Respirable Alpha

QUININE SULFATE DIHYDRATE, 106

Fluorescence
RADIOACTIVITY, 109-114

Americium

Barium

Carbon-14

Cesium-137/Barium-137m Point Source

Chlorine

Chromium

Cobalt

Curium

Environmental

Europium

Gallium

Gold

Human Liver

Human Lung

Hydrogen

Indium

Iodine

Iron

Krypton

Lead

Mercury

Mixed Radionuclides

Molybdenum

Natural Matrix

Nickel

Niobium

Peruvian Soil

Phosphorus

Plutonium

Polonium

Promethium

Radium

River Sediment

Rocky Flats Soil Number 1

Selenium

Strontium

Sulfur

Technetium

Thallium

Thorium

Tin

Uranium

Xenon

Ytterbium

RADIOGRAPHIC

X-ray Film Step Tablet, 125

RADIUM, 109-114

Radioactivity

RAINWATER, 59

Simulated

REFERENCE FUELS, see Fuels

REFLECTANCE, 107

(See also, Spectrophotometry)

Aluminum Mirrors

Aluminum Mirror with Wedge

Black Porcelain Enamel

Directional-Hemispherical

First Surface Mirror

Gold Mirror

Mirrors

Near IR

Second Surface Mirror

White Ceramic Tile

White Opal Glass

REFRACTIVE INDEX, 108

Ellipsometrically Derived, $\mathrm{SiO}_{2}$ on $\mathrm{Si}$ 
Isooctane

Silicone Liquids

Soda-Lime Glass

Toluene

2,2,4-Trimethylpentane

REFRACTORIES, 80

RESIDUAL RESISTIVITY RATIO, 119

RESISTIVITY

Electrical, 118

RRR, 119

Silicon, Eddy Current Testers, 118

Silicon, Spreading Resistance, 118

Thermal, 104

RHENIUM, 109-114

Isotopic

Spectrometric Solution, 45

RHODIUM

Spectrometric Solution, 45

RICE FLOUR, 51

RIVER SEDIMENT

Environmental, 62

PCB's in, 63

Radioactivity, 113

ROCKS

Basalt, 80

Obsidian, 80

Phosphate, 74, 77

ROCKY FLATS SOIL, 113

Radioactivity

RRR, see Residual Resistivity Ratio

RUBBER, 121

Butyl

Isobutylene-Isoprene (Butyl)

Styrene Butadiene

RUBBER COMPOUNDING MATERIALS, 121

Channel Black

Gas Furnace Black

Mercaptobenzothiazole

n-Tertiary-Butyl-2

Oil Furnace Black

Stearic Acid

Sulfur

Zinc Oxide

RUBIDIUM

Isotopic, 88

Melting Point, 102

Spectrometric Solution, 45

RUTHENIUM

Spectrometric Solution, 46

\section{SAMARIUM}

Spectrometric Solution, 46

SAND, GLASS, 79

SCANDIUM

Spectrometric Solution, 46

SCANNING ELECTRON MICROSCOPE

(SEM), 90

SEM Magnification

SEM Performance

SCHEELITE ORE, 75

SEDIMENT

Estuarine, 62
Marine, Organics in, 63

PCB's in, 63

River, 62

River (Radioactivity), 113

SELENIUM

Intermediate Purity, 40

Radioactivity, 109-114

Spectrometric Solution, 46

SEM, see Scanning Electron Microscope

SEMICONDUCTOR MANUFACTURING,

SILICA

Silica Refractory, 81

Thermal Expansion, 104

SILICON

Carbide, 81

Density, 96

Eddy Current, 118

Isotopic, 88

Metallo-Organic, 72

Resistivity, 118

Silicon Metal, 26

Spectrometric Solution, 46

X-ray Powder Diffraction, 117

SILICON DIOXIDE

Thickness and Refractive Thickness, 93

SILICONE, 108

SILVER

Isotopic, 88

Metallo-Organic, 72

Microprobe, 41, 42

Spectrometric Solution, 46

Vapor Pressure, 102

SIZING, 122

Glass Beads

Glass Spheres

Polystyrene Spheres

Portland Cement

SMOKE DENSITY, 128

Flaming (ABS plastic)

Nonflaming (cellulose)

SOCKETED BALL BAR, 123

SODA LIME GLASS, 83

Float

Sheet

SODIUM

-Bicarbonate (pH), 89

- Carbonate (pH), 89

-Chloride (Clinical), 49

Metallo-Organic, 72

-Oxalate (Reductometric), 44

-Pyruvate (Clinical), 49

Spectrometric Solution, 46

-Tetraborate Decahydrate (pH) [Borax], 89

SOLDER, 34

Lead-Base Alloy

SPECTRAL REFLECTANCE, see

Reflectance

SPECTROMETRIC SOLUTIONS, 45

Aluminum

Antimony

Arsenic

Barium

Beryllium

Bismuth

Boron 
Cadmium

Calcium

Cerium

Cesium

Chromium

Cobalt

Copper

Dysprosium

Erbium

Europium

Gadolinium

Gallium

Germanium

Gold

Hafnium

Holmium

Indium

Iridium

Iron

Lanthanum

Lead

Lithium

Lutetium

Magnesium

Manganese

Mercury

Molybdenum

Neodymium

Nickel

Niobium

Palladium

Phosphorus

Platinum

Potassium

Rubidium

Ruthenium

Samarium

Scandium

Selenium

Silicon

Silver

Sodium

Strontium

Sulfur

Tantalum

Tellurium

Terbium

Thallium

Thorium

Thulium

Tin

Titanium

Tungsten

Uranium

Vanadium

Ytterbium

Yttrium

Zinc

Zirconium

SPECTROPHOTOMETRY, 106

(See also, Reflectance)

Fluorescence Corrected Emission Spectrum

Glass Filters (Visible)

Liquid Absorbance Filters (UV and Visible)

Metal-on-Quartz Filters (UV and Visible)
Potassium Dichromate (UV Absorbance)

Potassium Iodide Stray Light

Quinine Sulfate Dihydrate (Fluorescence)

Stray Light

Ultra Violet

Visible

SPECULAR REFLECTANCE, see

Reflectance

STAINLESS STEEL

Chip Form, 19

Pitting/Crevice Corrosion, 116

Solid Form, 24

Thermal Expansion, 104

STEARIC ACID, 121

STEELMAKING ALLOYS, 26

Ferrochromium, High-Carbon

Ferrochromium, Low Carbon

Ferrochromium Silicon

Ferromanganese, High-Carbon

Ferroniobium

Ferrophosphorous

Ferrosilicon (48-Si)

Ferrosilicon (73-Si)

Ferrosilicon (75-Si)

STEEL, CHEMICAL COMPOSITION

Chip form, 15

Gasimetric, 39

Solid form, 20

STEP TABLET, 125

Photographic

Radiographic

$\mathrm{X}$-ray

STEP TEST, 115

Potential \& Thickness Step

STRONTIUM

Carbonate, 44, 88

Isotopic, 44, 88

Metallo-Organic, 72

Radioactivity, 109-114

Spectrometric Solution, 46

STYRENE BUTADIENE, 121

Rubber

SUCCINONITRILE, 102

Triple Point Cell

SUCROSE, 44, 108

Primary Chemical

SULFATE

Anion Solution, 48

SULFUR

-in Base Oil, 72

-in Coal, 61

-in Fossil Fuels, 61

-in Kerosene, 61

-in Lubricating Oil, 72

Rubber Compounding Material, 121

Spectrometric Solution, 46

SULFUR DIOXIDE

Analyzed Gas, 54

Permeation Tubes, 57

SUPERCONDUCTING

Thermometric Fixed Point Device, 101

SURFACE AREA, 123

SURFACE FINISH, 124

Sinusoidal Roughness 
TITANIUM

Alloys, 37

-Dioxide, 80

Spectrometric Solution, 46

Unalloyed Titanium, 37

Unalloyed Titanium for Gases, 39

TANTALUM

Spectrometric Solution, 46

TAPE ADHESION TESTING

Linerboard, 128

TECHNETIUM, 109-114

Radioactivity

TELLURIUM

Spectrometric Solution, 46

TERBIUM

Spectrometric Solution, 46

TETRACHLOROETHYLENE

Analyzed Gas, 54

Permeation Device, 57

TETRAHYDROCANNABIONOL IN

FREEZE-DRIED URINE, 68

THALLIUM

Isotopic, 88

Radioactivity, 109-114

Spectrometric Solution, 46

THC IN URINE, 68

THERMAL ANALYSIS

Differential Scanning Calorimetry, 100

Differential Thermal Analysis, 101

THERMAL CONDUCTIVITY, 104

Fibrous Glass

Graphite

Iron

Stainless Steel

Tungsten

THERMAL EXPANSION, 104

Borosilicate Glass

Fused Silica

Stainless Steel

Tungsten

THERMAL RESISTANCE, 104

Fumed Silica Board

Glass Batt

Glass Blanket

Glass Board

THERMOGRAVIMETRY, 101

THERMOMETER, 103

Clinical Laboratory

THERMOMETRIC FIXED POINTS, 103

THIANTHRENE, 99

Calorimetric

THICKNESS

Coating, 92

Ellipsometrically Derived, $\mathrm{SiO}_{2}$ on $\mathrm{Si}, 93$

Step Test, Potential, 115

THORIUM, 109-114

Radioactivity

Spectrometric Solution, 46

THULIUM

Spectrometric Solution, 46

TIME SERVICE DISKETTE, 127

TIN

Enthalpy, 97, 98, 100

Freezing Point, 102

Metallo-Organic, 72

Radioactivity, 109-114

Spectrometric Solution, 46

ULTRASONICS, 97

Acoustic Emission Transducer

Power Standard

URANIUM

Radioactivity, 109-114

UREA

Spectrometric Solution, 46

Calorimetric, 99

Clinical, 49, 50

Microchemical, 44 
URIC ACID, 49

Clinical

URINE, FREEZE-DRIED

Cocaine Metabolite, 68

Cotinine, 68

Fluorine, 68

Industrial Hygiene, 68

Mercury, 68

THC in, 68

Toxic Metals, 68

\author{
VANADIUM \\ -in Crude Oil, 59 \\ -in Residual Fuel Oil, 59 \\ Metallo-Organic, 72 \\ Spectrometric Solution, 46 \\ VAPOR PRESSURE, 102 \\ Cadmium \\ Gold \\ Silver \\ VICKERS \\ Microhardness, 96 \\ VISCOSITY, 94, 95 \\ VITAMINS \\ -in Coconut Oil, 52 \\ VMA (see Clinical)
}

\section{WASPALOY, 35}

WATER ANALYSIS

(See also, Electrolytic Conductance)

Acid Rain, 59

Anion Solutions, 48

Halocarbons, 63, 64

Mercury, 59

Rainwater, Simulated, 59

Spectrometric Solution, 46

Trace Elements, 62

WAVELENGTH, 106

Didymium Glass Filter

Holmium Oxide Solution

Near IR Reflectance

WEAR, ABRASIVE

Tool Steel, 115
WEAR-METALS IN OIL, 73

WHEAT FLOUR, 51

WHITE CERAMIC TILE

Reflectance, 122

WHITE OPAL GLASS

Reflectance, 122

WINE, STABILIZED, 52

\author{
XENON, 109-114 \\ X-RAY \\ Radioactivity \\ Diffraction, 116 \\ Fluorescence, Glass Target, 116 \\ Step Tablet, 125 \\ Thin-Glass Film on Polycarbonate Filter, \\ 69
}

\author{
YEAST, BREWERS, 51 \\ YTTERBIUM \\ Radioactivity, 109-114 \\ Spectrometric Solution, 46 \\ YTTRIUM \\ Spectrometric Solution, 46
}

ZINC

Alloys, 38

Enthalpy, 97, 98, 100

Freezing Point, 102

High Purity, 40

Metallo-Organic, 72

-on Filter Media, 71

Oxide, Rubber Compounding Material, 121

Spectrometric Solution, 46

Spelter, 38

ZIRCONIUM

Alloys, 38

Spectrometric Solution, 46 


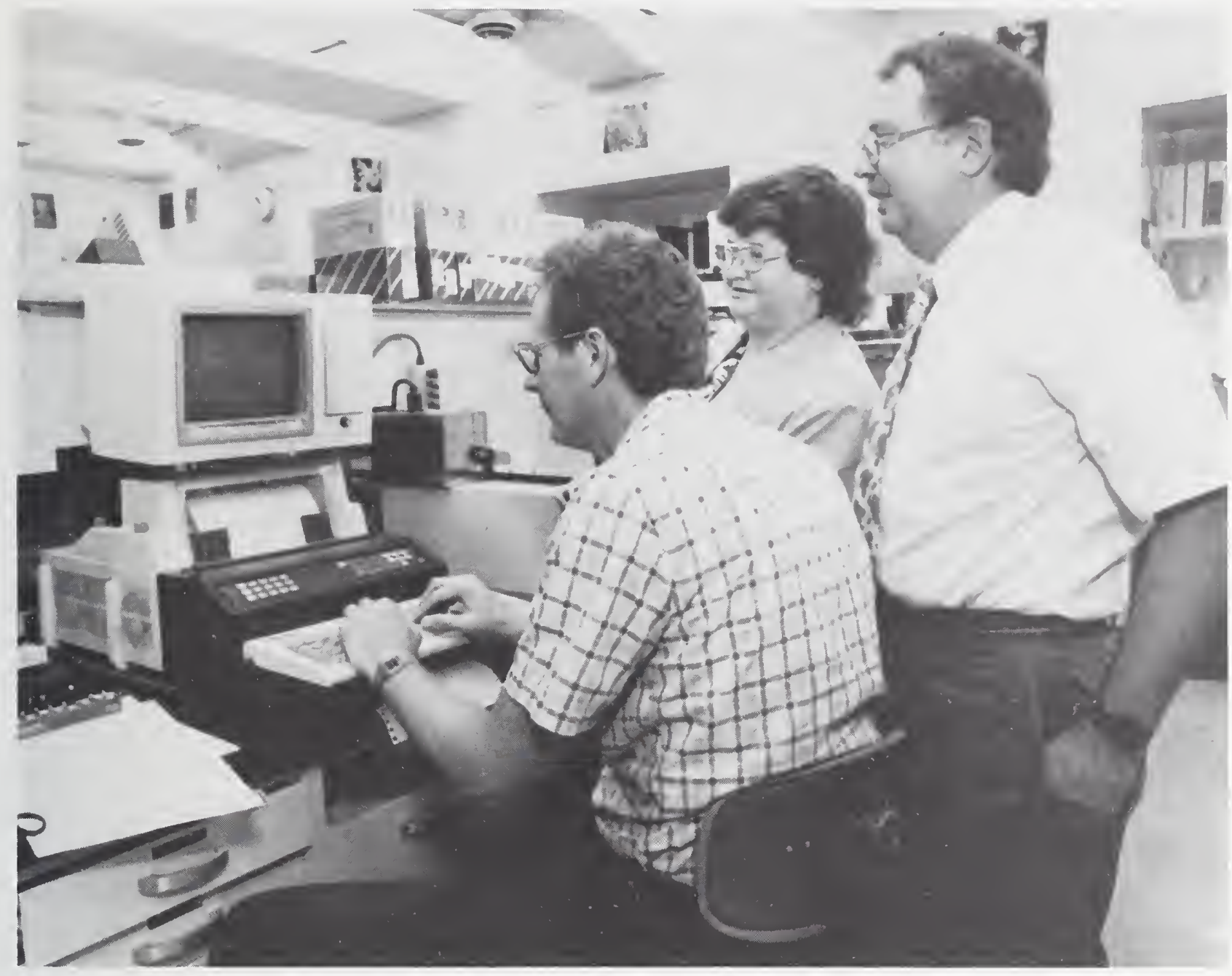

Stephen Wise, Reenie Parris and Stephen Chesler (left to right), of the Organic Analytical Research Division, evaluate chromatographic data for measurement of organic contaminants in an environmental SRM. 

U.S. DEPARTMENT OF COMMERCE

Mational Institute of Standards and Techinology

Standard Reference Materials Program

Bldg. 202, Room 204

Gaithersburg, MD. 20899

Retum and Forwarding Postage Guaranteed

Address Correction Requested

Official Business

Penalty for Private Use $\mathbf{\$ 3 0 0}$

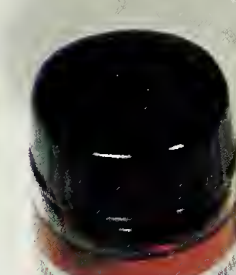

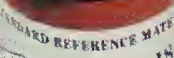

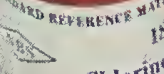

Chlorit

Ches or $\mathrm{c}^{\mathrm{v}}$
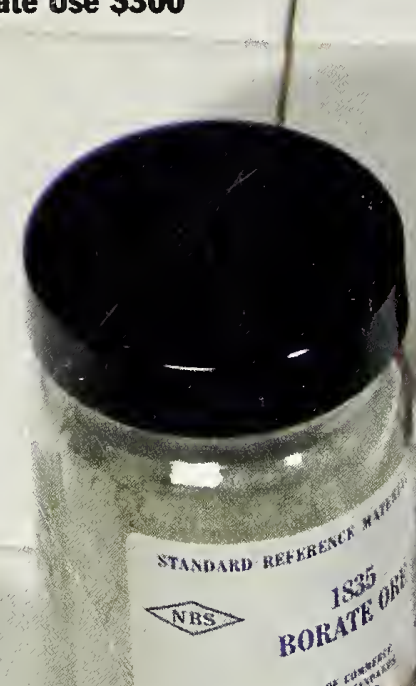

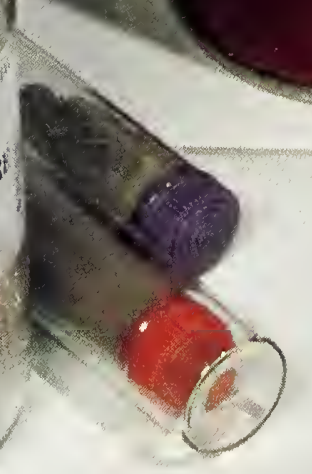

